Historic, Archive Document

Do not assume content reflects current scientific knowledge, policies, or practices. 


\section{Range Plants of Arizona and New Mexico} Names, Symbols, and Notations

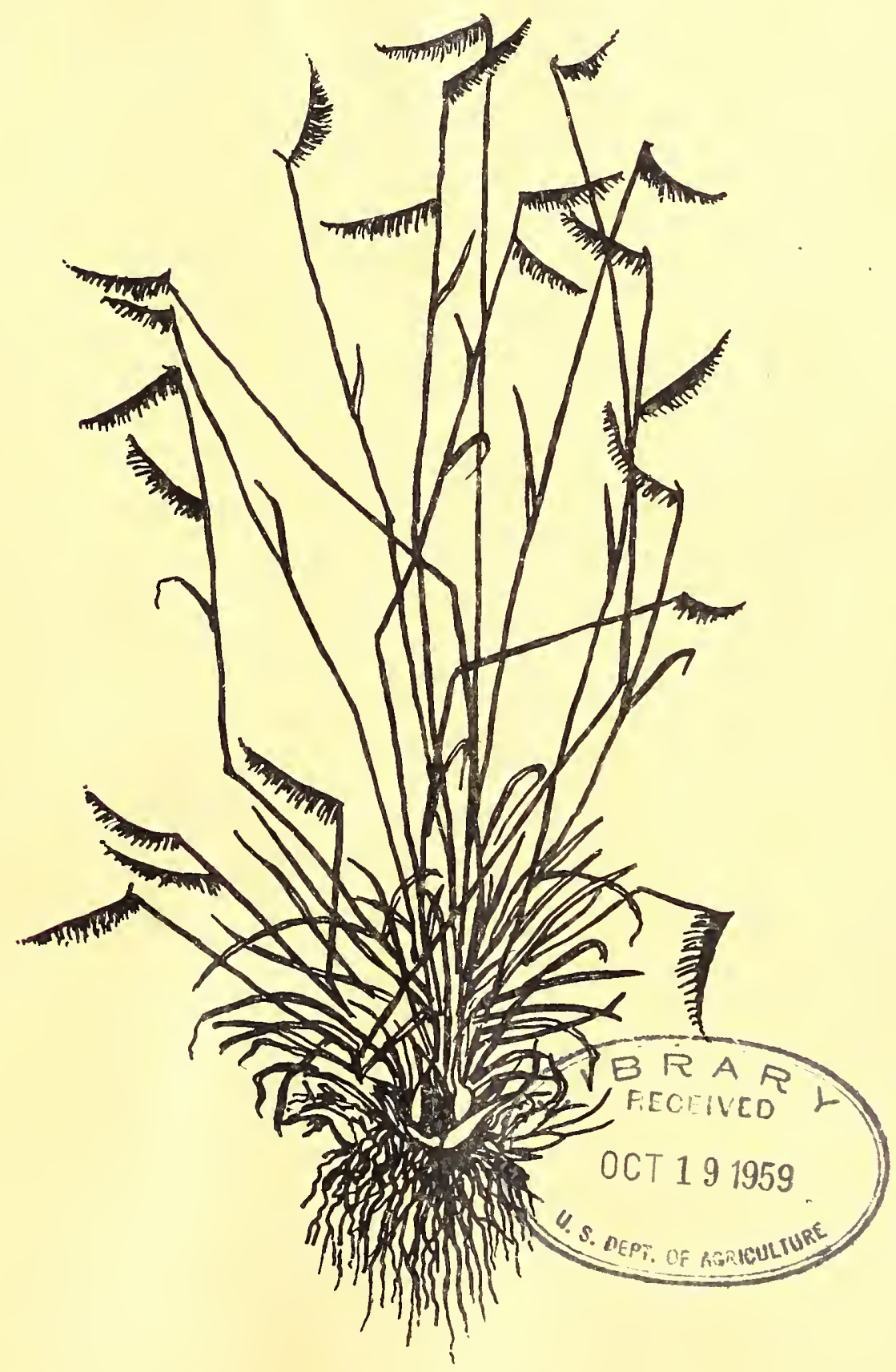

Rocky Mountain Forest and Range Experiment Station Fort Collins, Colorado Raymond Price, Director

Forest Service U. S. Department of Agriculture (For In-Service Use) 


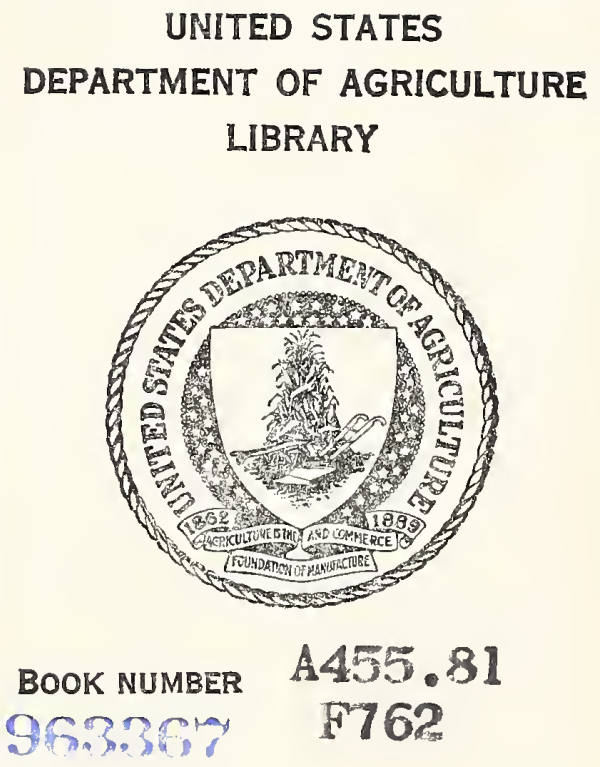

USDA National Agricultural Library

NAL Building

10301 Baltimore Blvd.

Beltsville, MO 20705-2351 
PIANT SYMBOL LIST FOR

RANGE PLANIS OF ARIZONA AND NEW MEXICO

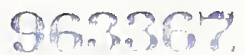

Introduction
USDA National Agricultural Library

NRL Building

10301 Baltimore Blvd.

Bellsville, MD 20705-2351

This list of cormon and botanical names has been compiled from records of plants collected by the U. S. Forest Service in Arizona and New Mexico. It is intended to provide a tabulation of (I) accepted botanical names for the most common species, (2) common names for these species, and (3) standard symbols for use in field records.

The botanical names follow the International code which is the code followed by the U. S. Department of Agriculture. Appropriate cross-reference and synonymy are given where names were different under the formerly used American Code. Symonyms also have been added, as necessary, to correlate with "Arizona Flora"1y by Kearney and Peebles, Ed. 2, 1951, and "A Flora of Arizona and New Mexico" 2/ by Tidestrom and Kittell, 194I, since these two manuals are most likely to be used by research workers in the area.

One to several common names are given for most plants. In the first column of common names is the name or names that have been used in the past in the Southwest. In the second colum is the common name from "Standardized Plant Names," 1942, $3 /$ or the "Check List of Native and Naturalized Trees of the United States," 4 if these names are different from those in the first column. Generally the names in the second colum are preferable to those in the first. For a few plants, names in the second column are marked by "\%". These are suggested names to replace those in use where they are in conflict with accepted names but for which names are not listed in Standardized Plant Names.

Symbols are four letters for genera and species and five letters for varieties. The rules used in assigning symbols are as follows:

1. For genera the first four letters are used and all letters are capitalized.

2. For species the first two letters of the generic name followed by the first two letters of the species name are used and only the first letter capitalized.

If "Arizona Flora" by Thomas H. Kearney and Robert H. Peebles. Berkeley, California. 1951.

2/ "A Flora of Arizona and New Mexico" by Ivar Tidestrom and Sister Teresita Kittell. Washington, D. C. 1941.

3/ "Standardized Plant Names" by Harlan P. Kelsey and

William A. Dayton. Harrisburg, Pa. 1942.

4/ "Check List of Native and Naturalized Trees of the United
States." U. S. Dept. Agr. Handbook 4I. 1953. 


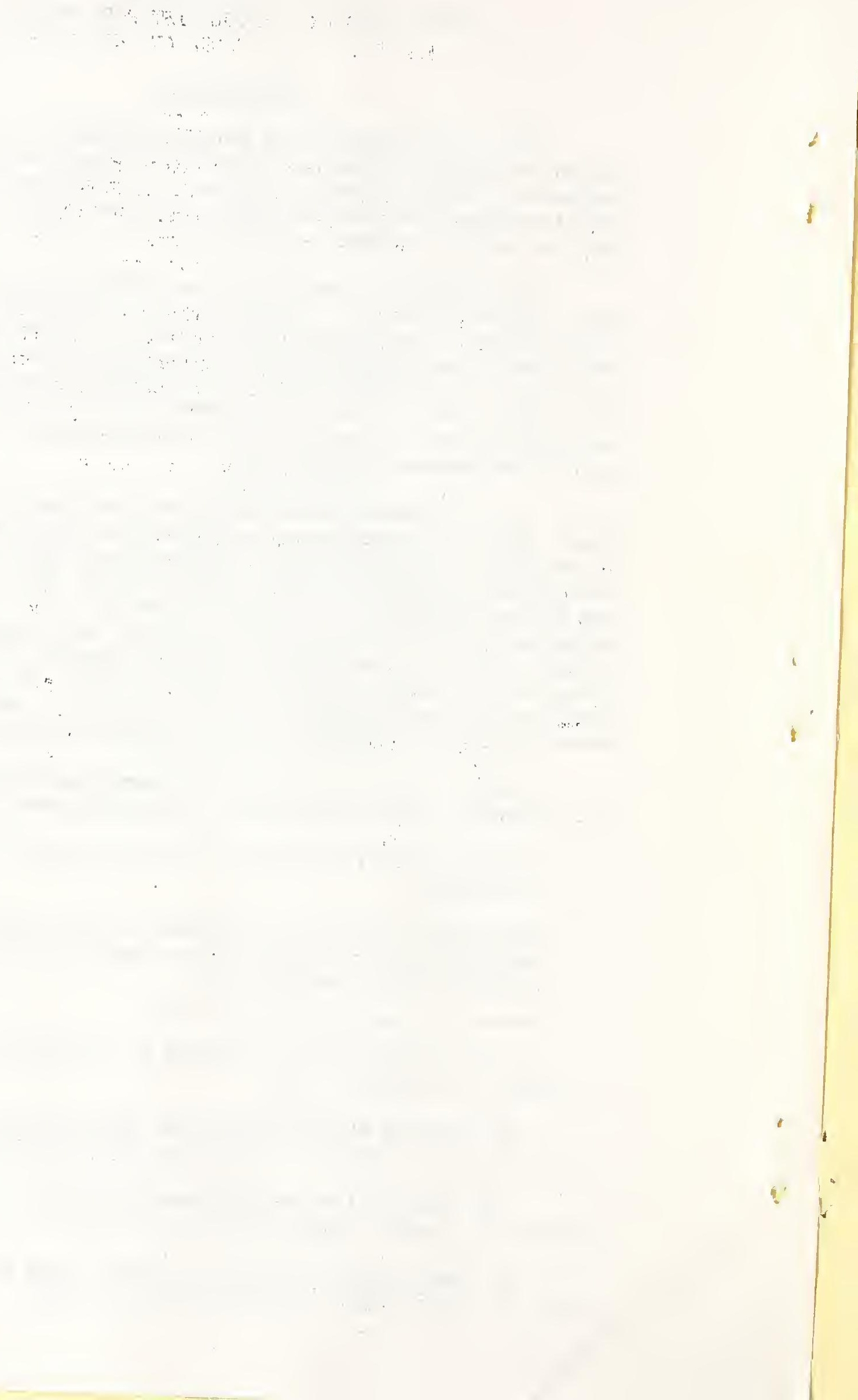


3. For varieties the first two letters of the generic name, the first two letters of the species name, and the first letter of the varlety name are used and only the first letter capitalized.

4. Where duplication of symbols occurs, numbers are added to indicate such duplication. The number is part of the symbol.

Marks used in the list are as follows:

1. Symonyms are indicated by underlining.

2. Subspecies are indicated by "ssp" between the species and subspecies names or preceding the subspecies name when it is indented under the species name.

3. Varieties are indicated by indentation of several spaces under the species name or, in writing the varietal name on the same line as the species name, the word "var." is between the species and variety names. Under the International Code the variety should always be preceded by "var."

4. Forms are indicated by "forma" between the species and form names, or preceding the form name when it is indented several spaces under the species name. 
a. 4.

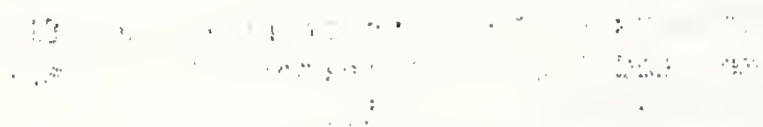

4

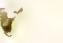


Aete Aegopogon tenellus (DC.) Trin. relaxgrass

$\begin{array}{lll}\text { ARIS } & \text { Aristida } & \text { three-awn } \\ \text { Arad } & \text { adscensionis I. } & \text { sixweeks three-awn } \\ \text { Arol } & \text { oligantha Michx. } & \text { prairie three-awn }\end{array}$

$\begin{array}{lc}\text { AvEN } & \text { Avena } \\ \text { Avba } & \text { barbata Brot. } \\ \text { Avfa } & \text { fatua I. } \\ \text { Avsa } & \text { sativa I. } \\ & \\ \text { BouT } & \text { Bouteloua } \\ \text { Boar } & \text { aristidoides (H.B.K.) Grise } \\ \text { Boba } & \text { barbata Iag. } \\ \text { Bopa } & \text { parryi (Fourn.) Griffiths } \\ \text { Bosi } & \text { simplex Lag. } \\ & \\ \text { BRoM } & \text { Bromus } \\ \text { Brbr-2 } & \text { brizaeformis Fisch. \& Mey. } \\ \text { Brca-2 } & \text { catharticus Vahl } \\ \text { Brja } & \text { japonicus Thunb. } \\ \text { Brmo } & \text { mollis L. } \\ \text { Brri } & \text { rigidus Roth } \\ \text { Brru-1 } & \text { mbens L. } \\ \text { Brte-1 } & \text { tectorum L. }\end{array}$

Cepa-1 Cenchrus pauciflorus Benth.

Chvi-1 Chloris virgata Swartz

ECHI-1 Echinochloa

Ecco colonum (L.) Iink

Eccr crusgalli (L.) Beauv.

Eccrz zelayensis (H.B.K.) Hitchc. oats

slender oat

wild oat

oat

grama

needle grama

sixweeks grama

Parry grama

mat grama

\section{chess}

rattlesnake chess

rescuegrass

Japanese chess

soft chess

ripgut grass

foxtail chess

cheatgrass

(downy chess)

field sandbur

feather fingergrass

cockspur

jungle-rice

barnyardgrass

alkali barnyardgrass oat

common oat showy chloris

brome

rattle brome

rescue brome

Japanese brome

ripgut brome

foxtail brome

(red brome)

cheatgrass brome

(downy brome)

mat sandbur

Jungle-rice

(shanwamillet) barnyardgrass

Elin Eleusine Indica (I.) Gaertn.

goosegrass 

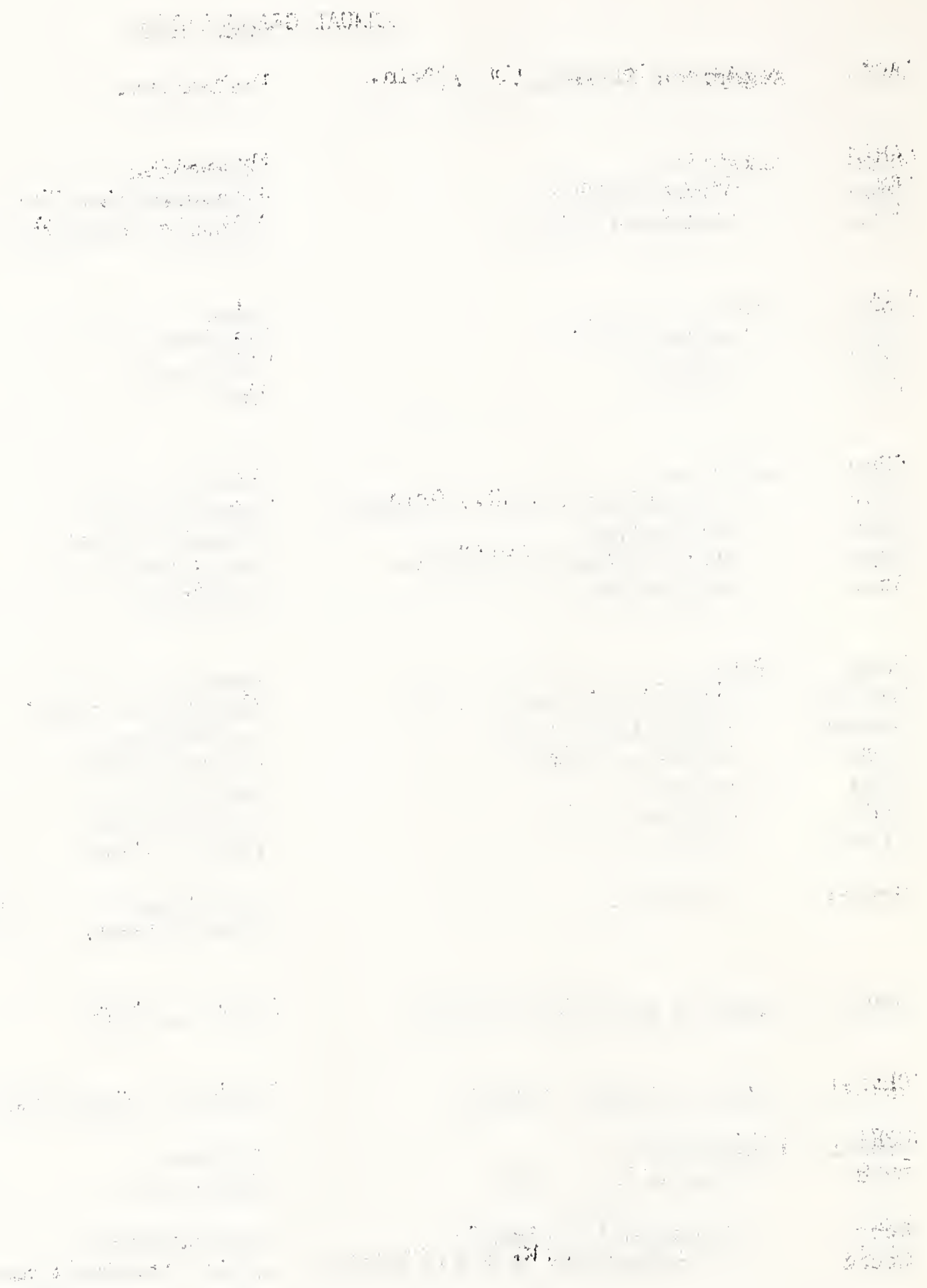

$$
\begin{aligned}
& \cdots, \cdots, \cdots
\end{aligned}
$$

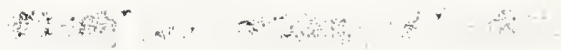

$$
\begin{aligned}
& \text { a }
\end{aligned}
$$




\section{ANNUAI GRASS LIST (continued)}

$\begin{array}{ll}\text { ERAG } & \text { Eragrostis } \\ \text { Erci-2 } & \text { cilianensis (All.) Lutati } \\ \text { Erdi-1 } & \text { diffusa Buckl. } \\ \text { Erlu } & \text { lutescens Scribn. } \\ \text { Erme } & \text { mexicana (Hornem.) Link } \\ \text { Erne } & \text { neomexicana Vasey } \\ \text { Erpe } & \text { pectinacea (Michx.) Nees } \\ \text { Erpi } & \text { pilosa (L.) Beauv. }\end{array}$

lovegrass

stinkgrass

spreading lovegrass

sixweeks lovegrass

Mexican lovegrass

New-Mexican lovegrass

Carolina lovegrass

India lovegrass

Ergr Eriochloa gracilis (Fourn.) Hitchc. southwestern cupgrass

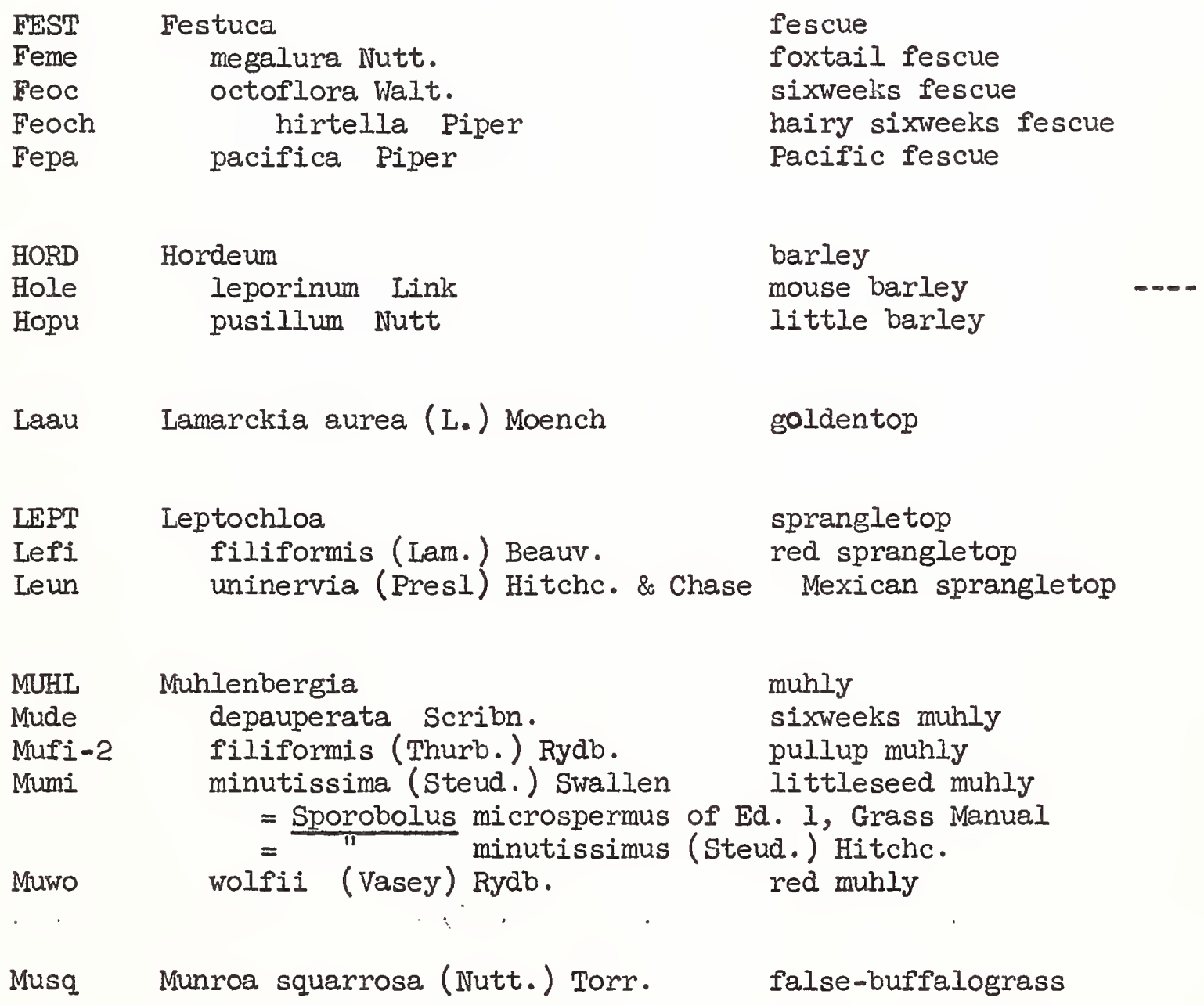
PANI Panicum
Paar
Paca
Pacao
Pafa
Pafar
Pahi
arizonicum Scribn. \& Merr. capillare I. occidentale Rydb. fasciculatum Swartz reticulatum (Torr.) Beal hirticaule Presl

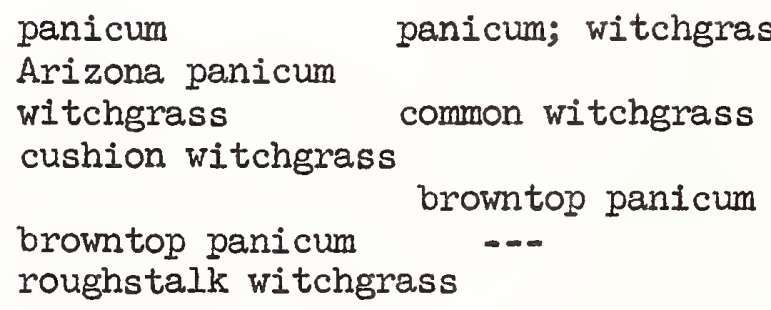

panicum

Arizona panicum

witchgrass

cushion witchgrass

browntop panicum common witchgrass browntop panicum roughstalk witchgrass
panicum; witchgrass




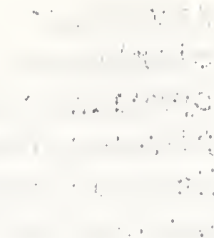


$\begin{array}{lcl}\text { POA } & \text { Poa } & \text { bluegrass } \\ \text { Poan-1 } & \text { annua I. } & \text { annual bluegrass } \\ \text { Pobi-1 } & \text { bigelovil Vasey \& Scribn. } & \text { Bigelow bluegrass }\end{array}$

Pomo Polypogon monspeliensis (L.) Desf. rabbitfoot grass

PUCC Puccinellia

Scba Schismus barbatus (I.) Thell. Mediterraneangrass

SETA

Segr

Selu

Sevi

SPOR

Spne-2

Sppa

Sppu

Spva

Trbe Tragus berteronianus Schult.

Trin Trisetum interruptum Buckl.
Setaria

grisebachii Fourn. viridis (I.) Beauv. lutescens (Weigel) Hubb. bristlegrass

Grisebach bristlegrass

yellow bristlegrass

green bristlegrass alkali-grass

dropseed

microspermus See Muhlenbergia minutissima minutissimus do.

neglectus Nash

patens Swallen

pulvinatus Swallen

vaginiflorus (Torr.) Wood

puffsheath dropseed

desert dropseed sixweeks dropseed poverty dropseed

spike bur-grass

annual trisetum rabbitfoot

polypogon
prairie tri- setum



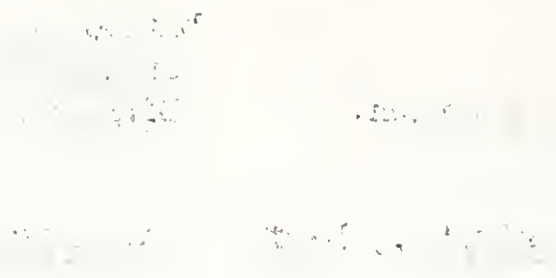


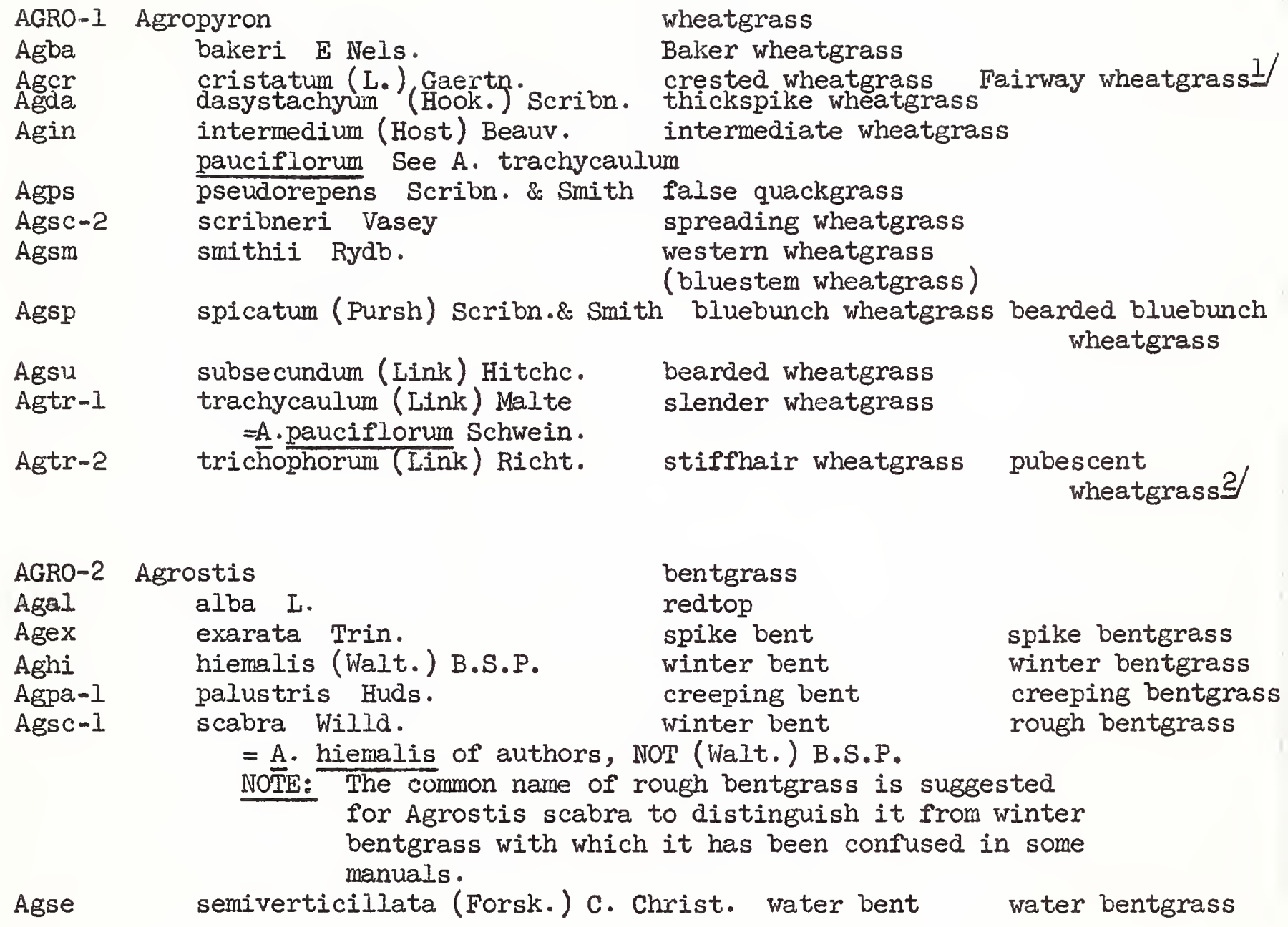

bentgrass

redtop

spike bent

winter bent

creeping bent

winter bent wheatgrass

spike bentgrass winter bentgrass creeping bentgrass rough bentgrass 


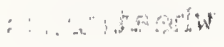

$$
\begin{aligned}
& \text { … } 4 \text { ? }
\end{aligned}
$$

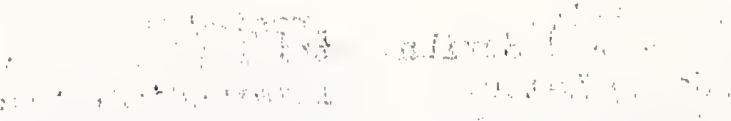
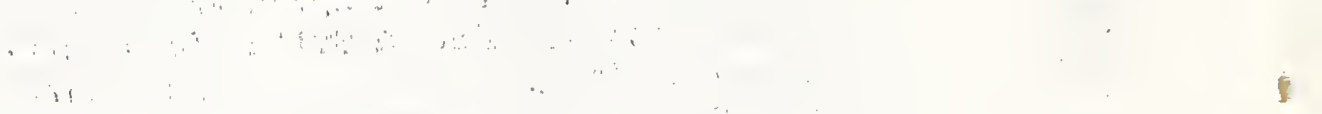

$+$ 


\section{AIDR-1 Andropogon}

$\begin{array}{ll}\text { Anba } & \text { barbinodis Lag. } \\ \text { Anci } & \text { cirratus Hack. } \\ \text { Ange } & \text { gerardil Vitman } \\ & \text { = A. furcatus Muhl. } \\ \text { Angl-I } & \text { glomeratus (Walt.) B.S.P. } \\ \text { Anha } & \text { hallii Hack. } \\ \text { Anhi } & \text { hirtiflorus (Nees) Kunth } \\ \text { Anhif } & \text { feensis (Fourn.) Hack. } \\ \text { Anis } & \text { ischaemum L. } \\ \text { Ansa } & \text { saccharoides Swartz } \\ \text { Ansc } & \text { scoparius Michx. }\end{array}$

bluestem

(beardgrass) (bluejoint grass) cane biuestem

Texas bluestem

big bluestem

bushy beardgrass bushy bluestem

sand bluestem

Santa Fe bluestem

Turkestan bluestem

silver beardgrass

little bluestem

\section{SantaFe bluestem \\ EastIndies bluestem \\ silver bluestem}

$\begin{array}{ll}\text { ARIS Aristida } & \\ \text { Arar-1 } & \text { arizonica Vasey } \\ \text { Arba } & \text { barbata Fourn. } \\ \text { Arca-1 } & \text { californica Thurb. } \\ \text { Ardi } & \text { divaricata Humb. \& Bonpl. } \\ \text { Arfe-1 } & \text { fendleriana Steud. } \\ \text { Argl-1 } & \text { glabrata (Vasey) Hitche. } \\ \text { Argl-2 } & \text { glauca (Nees) Walp. } \\ \text { Arlo-1 } & \text { longiseta Steud. } \\ \text { Aror } & \text { orcuttiana Vasey } \\ \text { Arpa-1 } & \text { pansa Woot. \& Standl. } \\ \text { Arpu-1 } & \text { purpurea Nutt. } \\ \text { Arte-1 } & \text { ternipes Cav. }\end{array}$

three-awn (dogtown grass, poverty grass, needlegrass)

Arizona three-awn

Havard three-awn

Mohave three-awn

poverty three-awn

Fendler three-awn

Santa Rita three-awn SantaRita three-awn blue three-awn

red three-awn

single three-awn

Wooton three-awn

purple three-awn

spidergrass

Arel Arrhenatherum elatius (L) Presl. Tali oatgrass

Besy Beckmannia syzigachne (Steud.) Ferm. American sloughgrass

BItr Blepharoneuron tricholepis (Torr.) Nash pine dropseed

(beardless bunchgrass)

BOUT Bouteloua

Bobr breviseta Vasey

Boch chondrosioides (H.B.K.) Benth.

Bocu curtipendula (Michx.) Torr.

Boel eludens Griffiths

Boer eriopoda Torr.

Bofi filiformis (Fourn.)Griffiths

Bogr gracilis (H.B.K.)Lag.

Bohi hirsuta Lag.

Bora radicosa (Fourn.)Griffiths

Boro rothrockii Vasey

Botr trifida Thurb grama

gyp grama

sprucetop grama

side-oats grama

Santa Rita grama

black grama

slender grama

blue grama

hairy grama

purple grama

Rothrock grama

red grama

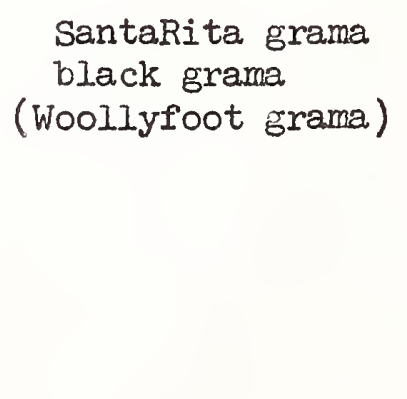


$\therefore \quad x+\Delta \div<\cdot 1$

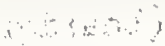

1 $\therefore \therefore$ son

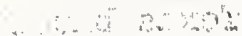

(.)

$\cdots \quad \because+1,+2,8$ 


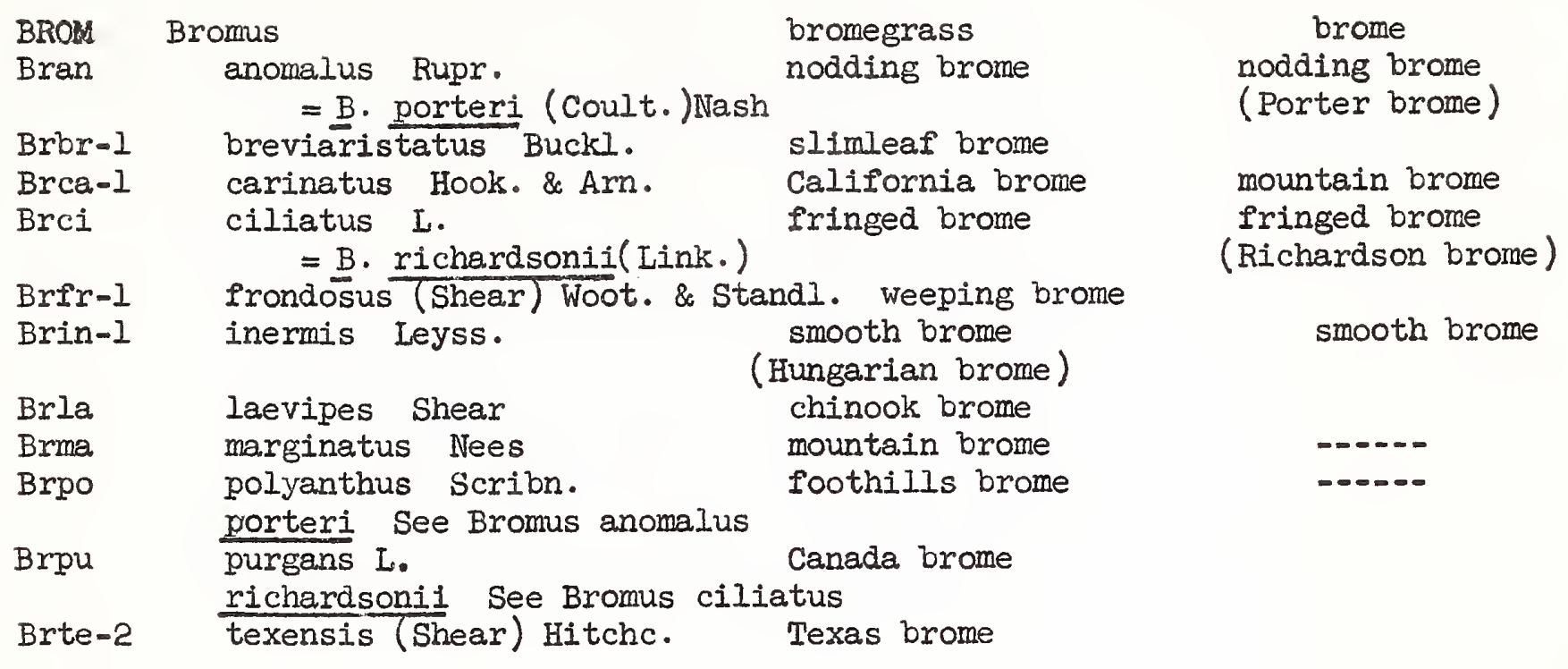

Buda Buchloe dactyloides (Nutt.) Engelm. buffalograss = Bulbilis dactyloides (Nutt.) Raf.
CALA-I Calamagrostis
Caca canadensis (Michx.) Beauv.
Cain-1 inexpansa A. Gray
Casc
scopulorum M. E. Jones
reedgrass
blue joint reedgrass
northern reedgrass
Jones reedgrass

Cagi Calamovilfa gigantea (Nutt.) Scribn. \& Merr. big sandreed big sandreed (giant reedgrass)

$\begin{array}{llll}\text { CHLO Chloris } & \text { chloris } & \begin{array}{c}\text { chloris } \\ \text { (windmillgrass) }\end{array} \\ \text { Chve } & \text { verticillata Nutt. } & \text { windmillgrass } & \text { tumble windmillgrass }\end{array}$

Cila-2 Cinna latifolia (Trevir.) Griseb. drooping woodreed

Cyda Cynodon dactylon (L.) Pers. Bermudagrass

Dagl Dactylis glomerata I. orchardgrass

DANT Danthonia

Dain intermedia Vasey

Dapa-I parryi Scribn.

Dasp-I spicata (I.) Beauv. oatgrass

timber oatgrass

Parry oatgrass

poverty oatgrass danthonia timber danthonia.

Parry danthonia poverty danthonia

Deca Deschampsia caespitosa (L.) Beauv. tufted hairgrass 


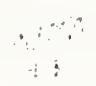


PERENNIAI GRASS IIST (continued)

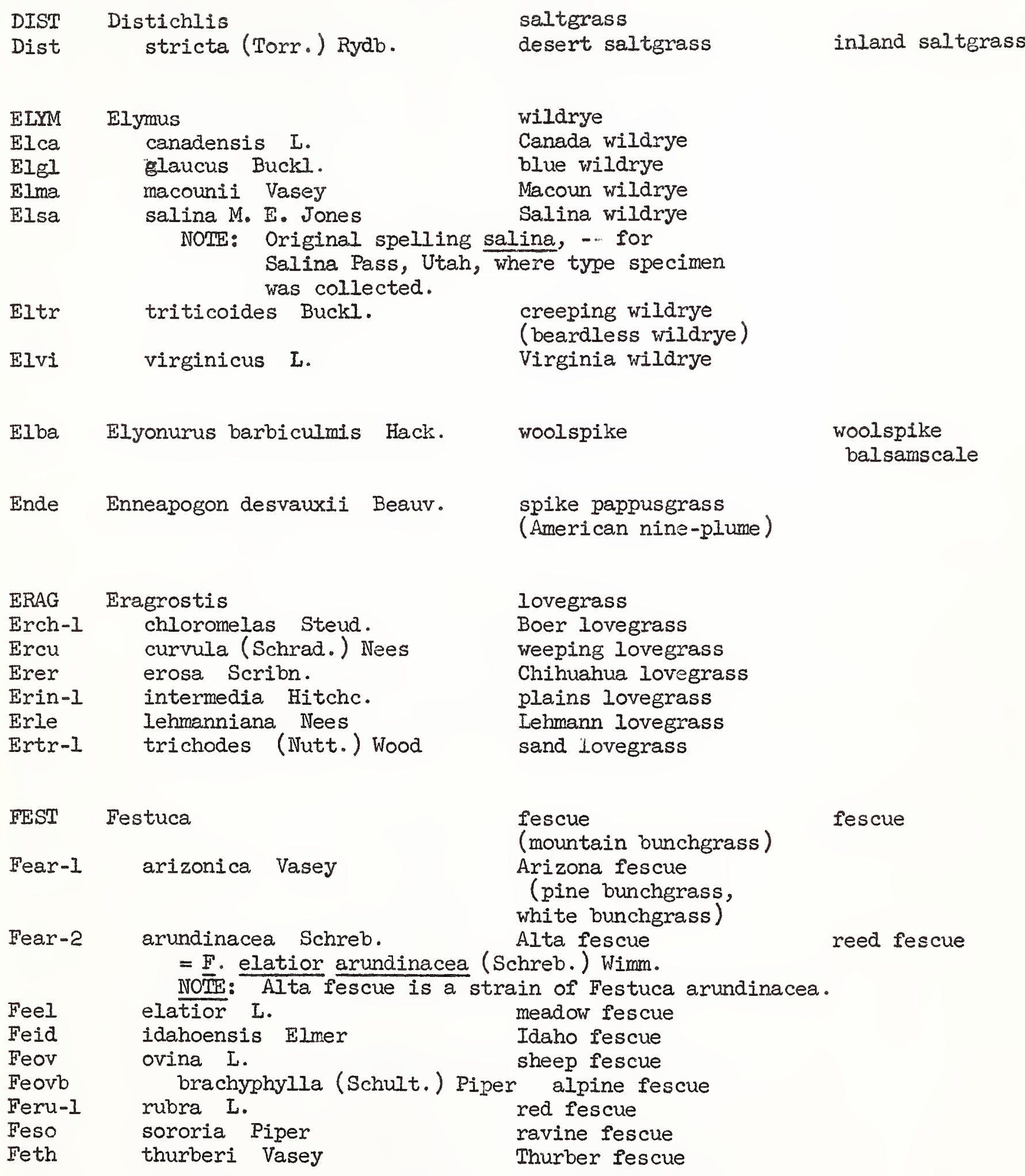




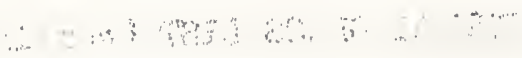

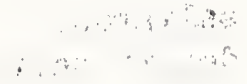


Perennial Grass List (continued)

$\begin{array}{lll}\text { GLWe } & \text { Glyceria } & \text { mannagrass } \\ \text { Glbo } & \text { borealis (Nash) Batchelder } & \text { northern mannagrass } \\ \text { Glel } & \text { elata (Nash) Hitchc. } & \text { tall mannagrass } \\ \text { Glgr } & \text { grandis S. Wats. } & \text { American mannagrass } \\ \text { Glpa } & \text { pauciflora Presl. } & \text { weak mannagrass } \\ \text { Glst. } & \text { striata (Iam.) Hitche. } & \text { fowl mannagrass }\end{array}$

Heco-l Heteropogon contortus (L.) Beauv. tanglehead

Hiod Hierochloë odorata (L.) Beauv. sweetgrass

$\begin{array}{lll}\text { HILA } & \text { Hilaria } & \text { hilaria } \\ \text { Hibe } & \text { belangeri (Steud.) Nash } & \begin{array}{l}\text { curlymesquite } \\ \text { (creepingmesquite) }\end{array} \\ \text { Hija } & \text { jamesii (Torr.) Benth. } & \text { galleta } \\ \text { Himu } & \text { mutica (Buckl.) Benth. } & \text { tobosa-grass } \\ \text { Hiri } & \text { rigida (Thurb.) Benth. } & \text { big galleta } \\ & & \text { (desert galleta) }\end{array}$

Hola Holcus lanatus L.

velvetgrass

cormon velvetgrass

HORD Hordeum

barley

(foxtail, squirreltail)

Hobr

brachyantherum Nevski

meadow barley

$=\mathrm{H}$. nodosum of authors, NOT $\mathrm{L}$.

$=$ H. nodosum boreale (Scribn. is Smith) Hitchc.

Hоји

Hono jubatum L. nodosum L. foxtail barley

-...-

Junegrass

(prairie Junegrass)

Leor Leersia oryzoides (L.) Swartz rice cutgrass

Ledu Leptochloa dubia (H.B.K.) Nees green sprangletop

Leco Leptoloma cognatum (Schult.) Chase fall witchgrass

LOLI

Lolium

Lomu multiflorum Lam. perenne L. ryegrass

Italian ryegrass

perenniai ryegrass

\% Suggested common name 


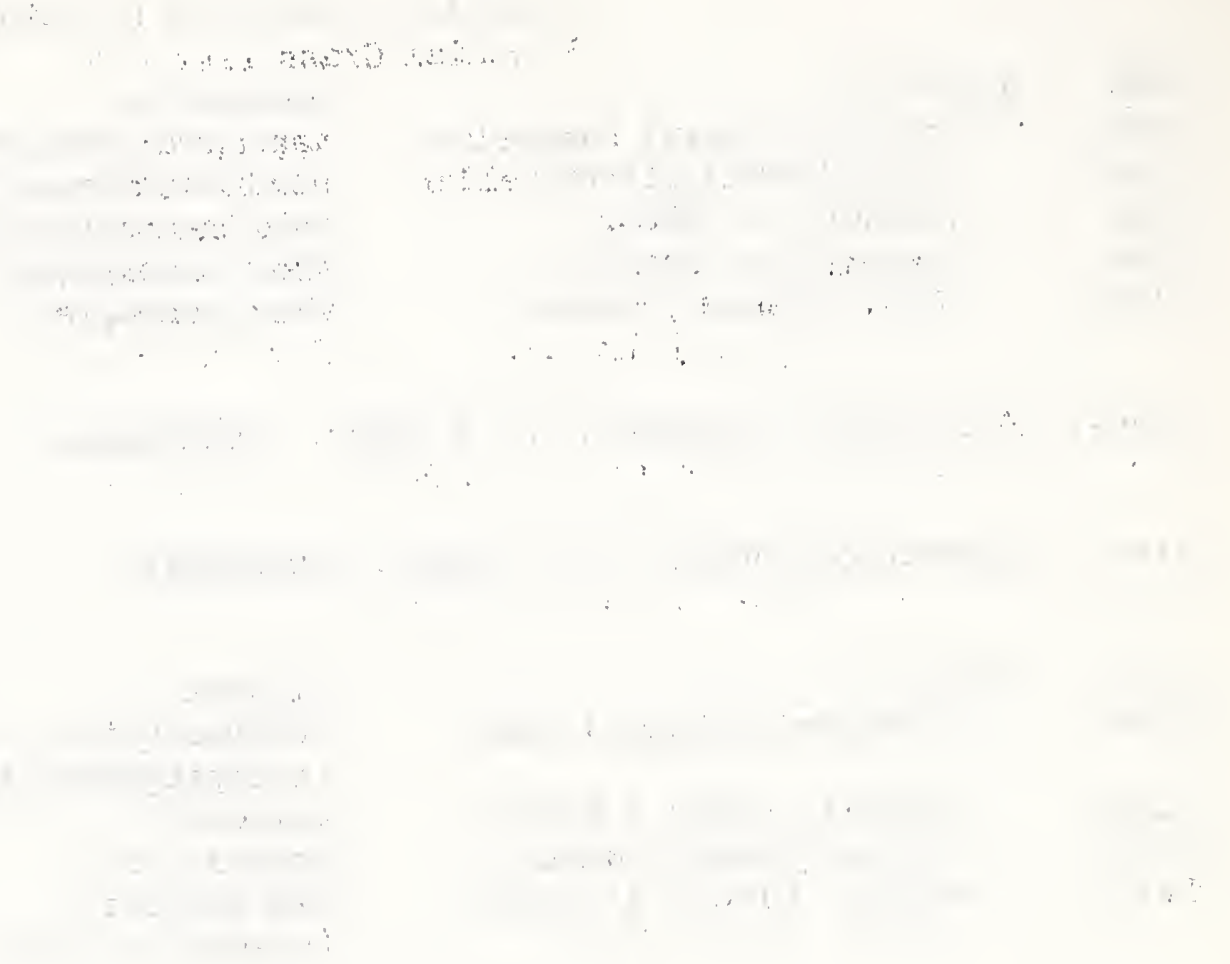




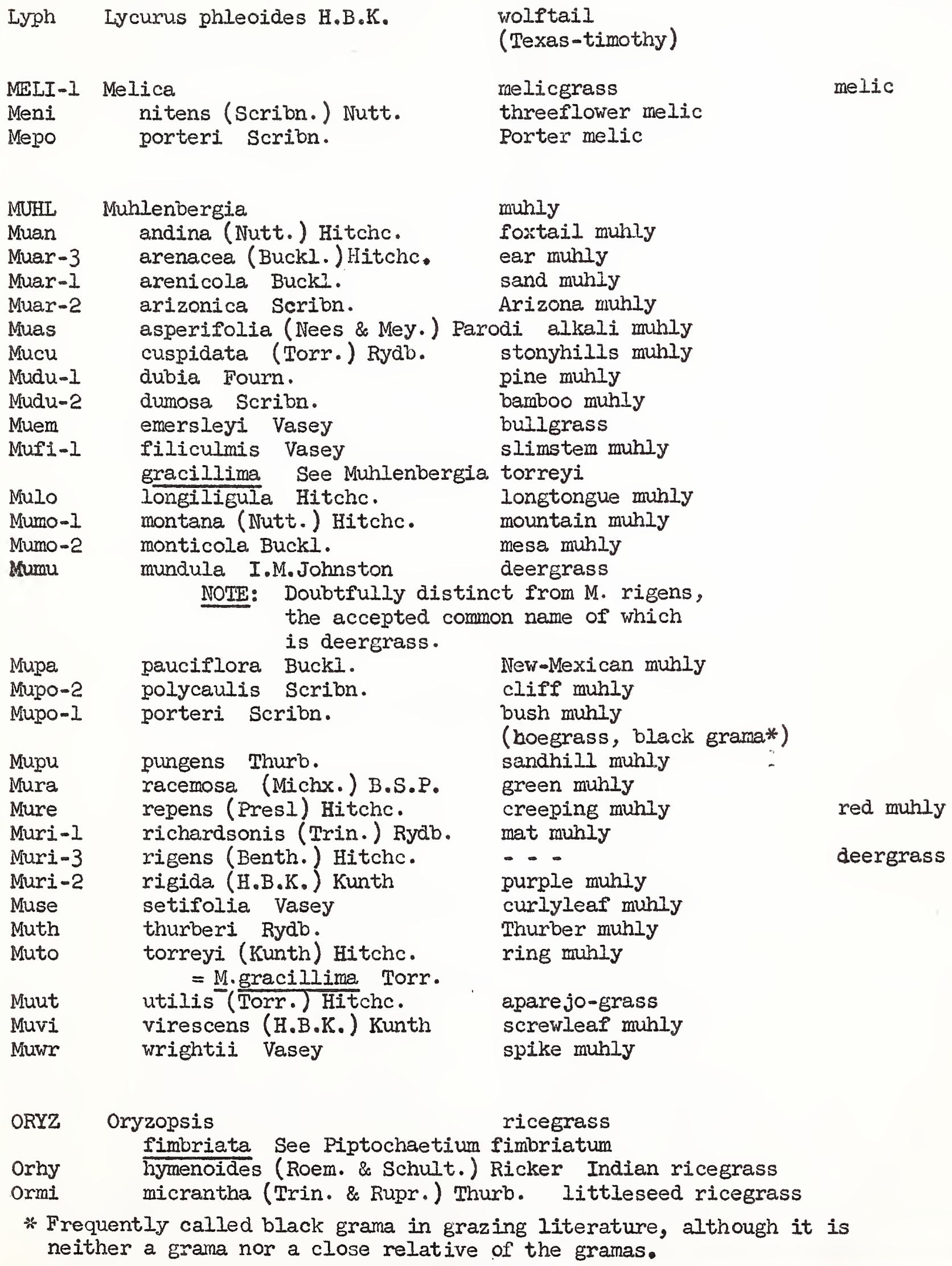

MUHL Muhlenbergia

mukly

Muan andina (Nutt.) Hitchc.

foxtail muhly

Muar -3 arenacea (Buckl.) Hitchc. ear muhly

Muar-1 arenicola Bucki. sand muhly arizonica Scrion. Arizona muhly

Muarasperifolia (Nees \& Mey.) Parodi alkali mubly

Mucu

Mudu-1 cuspidata (Torr.) Rydb. stonyhills muhly

Mudu-2 dubia Fourn. pine muhly

Muem dumosa Scribn. emersleyi Vasey

bamboo muhly

Mufi-I filiculmis Vasey bullgrass gracillima See Muhlenbergia torreyi

Mulo longiligula Hitchc.

longtongue muhly

Mumo-1

Mumo-2 montane (Nutt.) Hitchc. mountain muhly monticola Buckl. mesa muhly

urum mundula I.M.Johnston

deergrass

NOTE: Doubtfully distinct from M. rigens, the accepted cormon name of which is deergrass.

Mupa Bucki.

Mupo-2 polycaulis Scribn.

Mupo-1 porteri Scribn.

Mupu pungens Thurb.

Mura

Mure

Muri-1 racemosa (Michx.) B.S.P. repens (Presl) Hitchc. New-Mexican muhly

Muri-3 richardsonis (Trin.) Rydb. rigens (Benth.) Hitchc. rigida (H.B.K.) Kunth

Muri-2

Muse

Muth

Muto

Muut

Muvi

Muwr setifolia Vasey thurberi Rydb. torreyi (Kunth) Hitchc. $=$ M. gracillima Torr. utilis (Torr.) Hitche. virescens (H.B.K.) Kunth wrightii Vasey

cliff muhly

bush muhly

(boegrass, black grama*) sandhill muhly

green muhly creeping muhly

mat muhly

- -

purple muhly

curlyleaf muhly

Thurber muhly

ring mubly

aparejo-grass

screwleaf muhly

spike muhly

red muhly

deergrass

ORYZ Oryzopsis

ricegrass

fimbriata See Piptochaetium fimbriatum

Orhy hymenoides (Roem. \& Schult.) Ricker Indian ricegrass

Ormi micrantha (Trin. \& Rupr.) Thurb. littleseed ricegrass

* Frequently called black grama in grazing literature, although it is neither a grama nor a close relative of the gramas. 

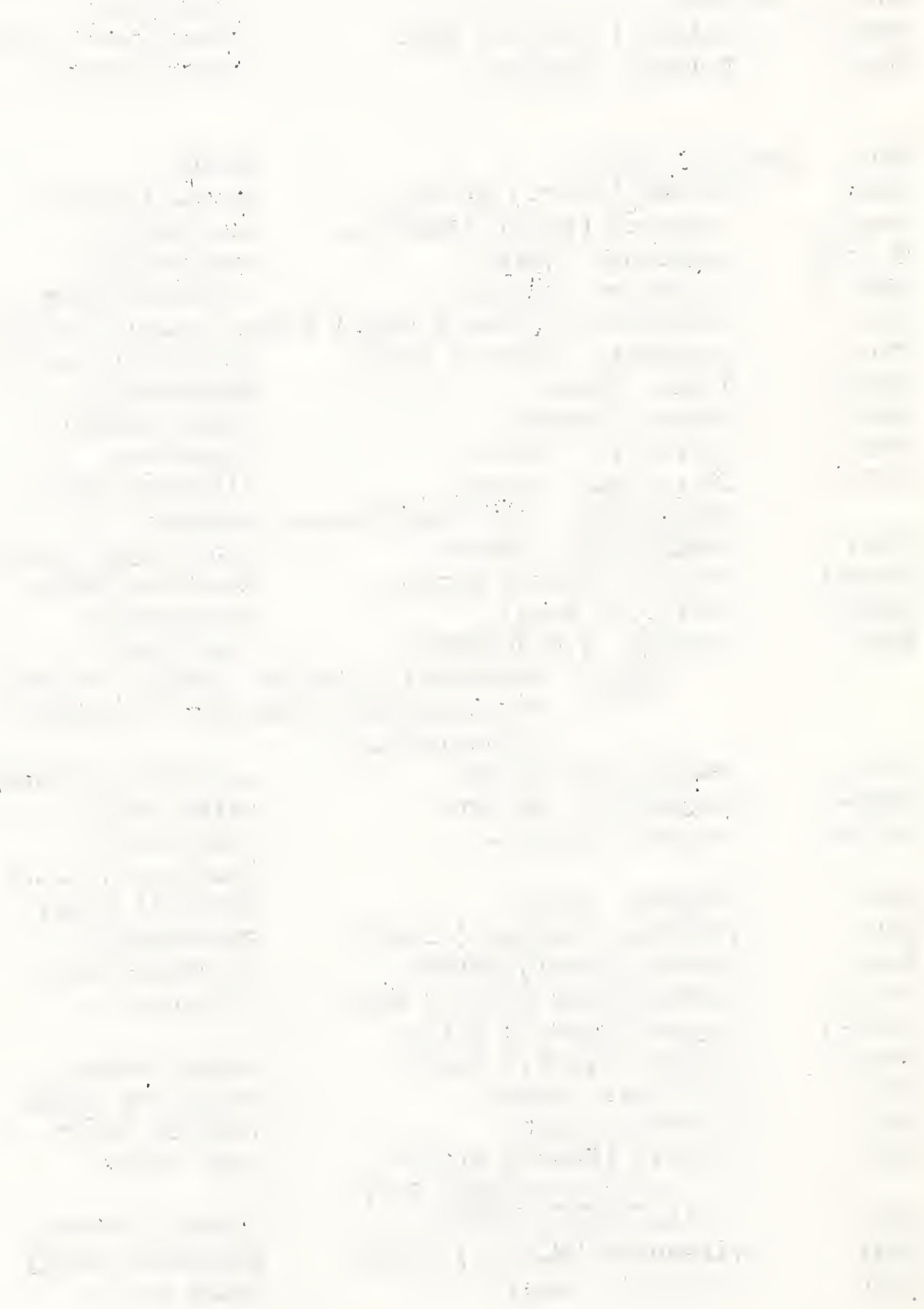


\section{FERENNIAL GRASS IIST (continued)}

$\begin{array}{ll}\text { PANI } & \text { Panicum } \\ \begin{array}{l}\text { Pabu } \\ \text { Pabum }\end{array} & \begin{array}{r}\text { bulbosum H.E.K. } \\ \text { minus Vasey }\end{array} \\ \text { Paha } & \text { hallii Vasey } \\ \text { Pahu } & \text { huachucae Ashe } \\ \text { Paob } & \text { obtusum H.B.K. } \\ \text { Pavi } & \text { virgatum I. }\end{array}$

Pamu Pappophorum mucronulatum Nees

Padi Paspalum distichum I.

Phar Phalaris arundinacea I.

PHIE
Phal
Phpr

Pheo

Pifi panicum

(panicgrass)

bulb panicum

little bulb panicum

Ha.lIs panicum

Huachuca panicum

vine-mesquite

switchgrass

feather pappusgrass panicum; witchgrass

little bulb panicurn whiplash pappusgrass

knotgrass

reed canarygrass

timothy

alpine timothy

timothy

common reed

POA Poa

Poam ampla Merr.

Poar-2 arctica R. Br.

Poar-1 arida Vasey

Poco-I compressa $I$.

Pofe

Poin-1

Pole

Polo

POOC

Popa

Popr

Pore

Poru

\section{fendleriana (Steud.) Vasey}

interior Rydb. leptocoma Trin. longiligula Scribn. \& Williams

occidentalis Vasey

palustris $I$. pratensis $\mathrm{L}$. reflexa Vasey \& Scribn. rupicola Nash big bluegrass

arctic bluegrass

plains bluegrass

Canada bluegrass

mutton biuegrass (muttongrass)

inland bluegrass

bog bluegrass

g bluegrass

New-Mexican bluegrass

fowl bluegrass

Kentucky bluegrass

nodding bluegrass

timberline bluegrass

Poin-2 Polypogon interruptus H.B.K. ditch polypogon 


\section{PERENNIAL GRASS LIST (continued)}

Scpa Schedonnardus paniculatus (Nutt.) Trel. $\begin{gathered}\text { tumblegrass } \\ \text { (Texas-crabgrass) }\end{gathered}$

Scpu Schizachne purpurascens (Torr.) Swallen false-melic false-melic

Scbr Scleropogon brevifolius Phil. burrograss

$\begin{array}{lll}\text { SETA } & \text { Setaria } & \text { bristlegrass } \\ \text { Sege } & \text { geniculata (Lam.) Beauv. } & \begin{array}{l}\text { knotroot bristlegrass } \\ \text { plains bristlegrass }\end{array} \\ \text { Sema } & \text { macrostachya H.B.K. } & \\ & & \text { squirreltail } \\ \text { SITA } & \text { Sitanion } & \text { bottlebrush squirreltail } \\ \text { Sihy } & \text { hystrix (Nutt.) J.G.Smith } & \text { big squirreltail } \\ \text { Siju } & \text { jubatum J.G. Smith } & \end{array}$

SORG Sorghastrum

Sonu nutans (I.) Nash

Indiangrass

yellow Indiangrass

Soha Sorghum halepense (I.) Pers. Johnsongrass

$\begin{array}{ll}\text { SPAR-1 } & \text { Spartina } \\ \text { Spgr } & \text { gracilis Trin. } \\ \text { Sppe-1 } & \text { pectinata Link }\end{array}$

SPHE Sphenopholis wedgegraiss

Spin-2 intermedia Rydb. slender wedgegrass

Spob obtusata (Michx.) Scribn. cordgrass

alkali cordgrass

prairie cordgrass

prairie wedgegrass wedgescale slender wedgescale prairie wedgescale

$\begin{array}{lcl}\text { SPOR } & \text { Sporobolus } & \text { dropseed } \\ \text { Spai } & \text { airoides Torr. } & \text { alkali sacaton } \\ \text { Spas } & \text { asper (Michx.) Kunth } & \text { tall dropseed } \\ \text { Spco } & \text { contractus Hitchc. } & \text { spike dropseed } \\ \text { Spcr } & \text { cryptandrus (Torr.) A. Gray sand dropseed } & \text { sas } \\ \text { Spfl } & \text { flexuosus (Thurb.) Rydb. } & \text { mesa dropseed } \\ \text { Spgi } & \text { giganteus Nash } & \text { giant dropseed } \\ \text { Spin-1 } & \text { interruptus Vasey } & \text { black dropseed } \\ \text { Spne-1 } & \text { nealleyi Vasey } & \text { gypgrass } \\ \text { Spte } & \text { texanus Vasey } & \text { Texas dropseed } \\ \text { Spwr } & \text { wrightii Munro } & \text { sacaton }\end{array}$


PERENIVIAI GRASS IIST ( continued)

$\begin{array}{lll}\text { STIP } & \text { Stipa } & \text { needlegrass; } \\ & \text { feathergrass } & \text { funeral needlegrass Mormon needlegrass } \\ \text { Star } & \text { arida M. E. Jones } & \text { Columbia needlegrass subalpine " } \\ \text { Stco-2 } & \text { columbiana Macoun } & \text { needle-and-thread } \\ \text { Stco-1 } & \text { comata Trin. \& Rupr. } & \text { Tweedy needlegrass } \\ \text { Stcoi } & \text { intermedia Scribn.\& Tweedy } & \text { Guadalupa needlegrass } \\ \text { Stcu } & \text { curvifolia Swallen } & \text { southwestern needlegrass } \\ \text { Stem } & \text { eminens Cav. } & \text { Letterman needlegrass } \\ \text { Stle } & \text { lettermanii Vasey } & \text { little-awn needlegrass } \\ \text { Stlo-1 } & \text { lobata Swallen } & \text { New-Mexican feathergrass } \\ \text { Stne } & \text { neomexicana (Thurb.) Scribn. } & \text { western needlegrass } \\ \text { Stoc } & \text { occidentalis Thurb. } & \text { Pringle needlegrass } \\ \text { Stpr } & \text { pringlei Scribn. } & \text { sleepygrass } \\ \text { Stro } & \text { robusta (Vasey) Scribn. } & \text { Scribner needlegrass } \\ \text { Stsc } & \text { scribneri Vasey } & \text { porcupinegrass } \\ \text { Stsp-1 } & \text { spartea Trin. } & \text { desert needlegrass } \\ \text { Stsp-2 } & \text { speciosa Trin. \& Rupr. } & \text { finestem needlegrass } \\ \text { Stte } & \text { tenuissima Trin. } & \text { green needlegrass } \\ \text { Stvi } & \text { viridula Trin. } & \end{array}$

Trse Trachypogon secundus (Presl) Scribn. crinkle-awn

Trca-1 Trichachne californica (Benth.) Chase cottontop Arizona cottontop = Trichachne saccharata Nash

TRID Tridens = Triodia tridens (cottongrass)

Tral-1 albescens (Vasey) Woot. \& Standl. white tridens

Trel elongatus (Buckl.) Nash rough tridens

Trgr grandiflorus (Vasey) Woot. \& Standl. largeflowered shortleaf tridens tridens

NOTE: Big-spikelet tridens is

also suggested as a common

name for this species.

\begin{tabular}{|c|c|c|c|}
\hline $\begin{array}{l}\text { Trmu } \\
\text { Trpi } \\
\text { Trpu }\end{array}$ & $\begin{array}{l}\text { muticus (Torr.) Nash } \\
\text { pilosus (Buckl.) Hitchc. } \\
\text { pulchellus (H.B.K.) Hitchc. }\end{array}$ & $\begin{array}{l}\text { slim tridens } \\
\text { hairy tridens } \\
\text { fluffgrass }\end{array}$ & 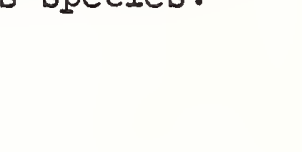 \\
\hline TRIS & Trisetum & trisetum & \\
\hline Trmo & montanum Vasey & mountain trisetum & $\begin{array}{c}\text { RockyMountain } \\
\text { trisetum }\end{array}$ \\
\hline $\begin{array}{l}\text { Trsp } \\
\text { Trwo }\end{array}$ & $\begin{array}{l}\text { spicatum (I.) Richt. } \\
\text { wolfii Vasey }\end{array}$ & $\begin{array}{l}\text { spike trisetum } \\
\text { Wolfs trisetum }\end{array}$ & \\
\hline
\end{tabular}





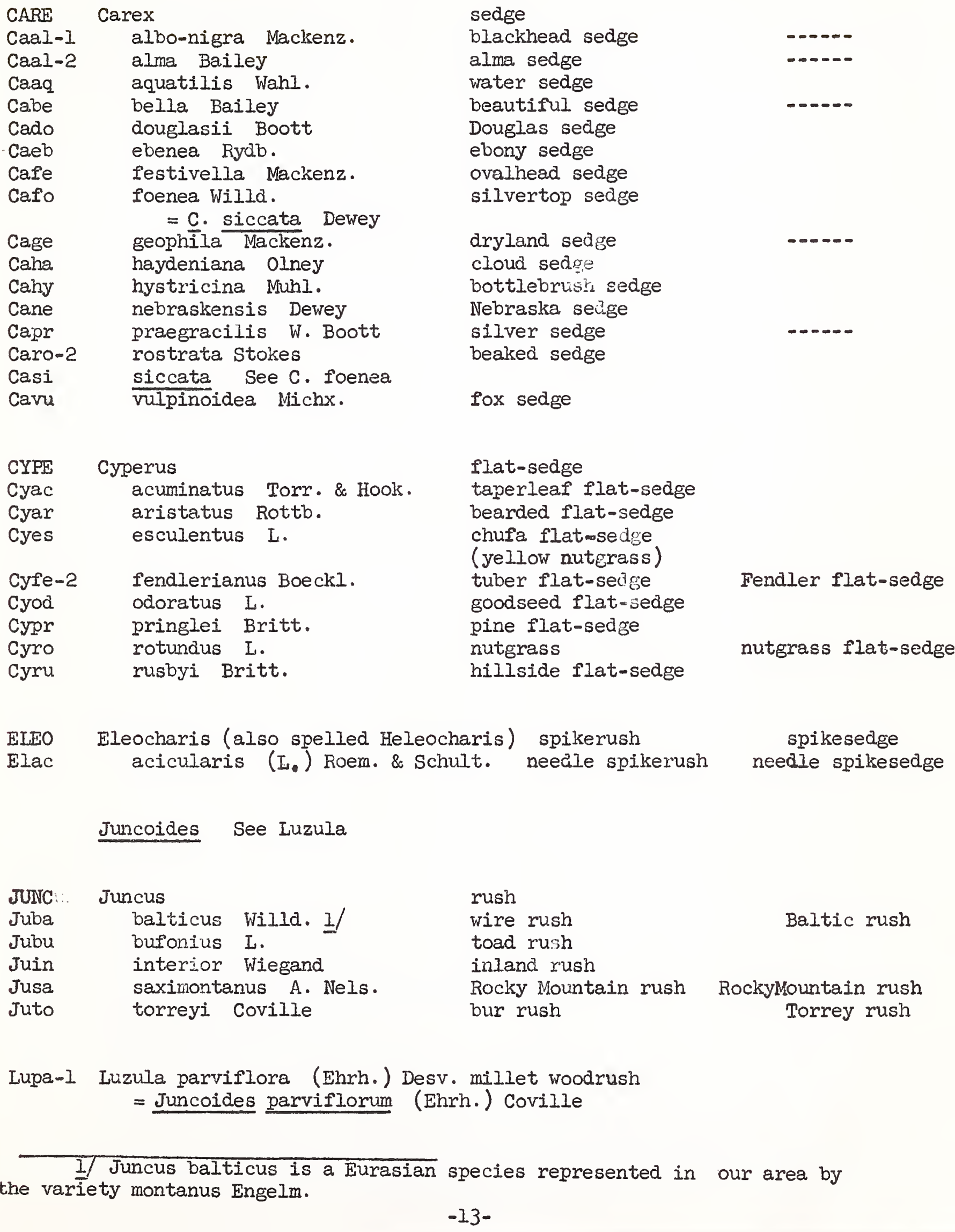




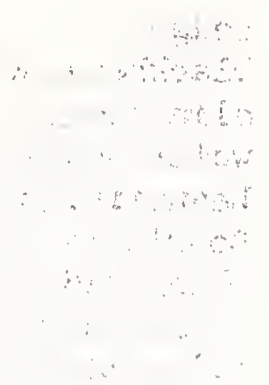


GRASSLIKE PLANTS (continued)

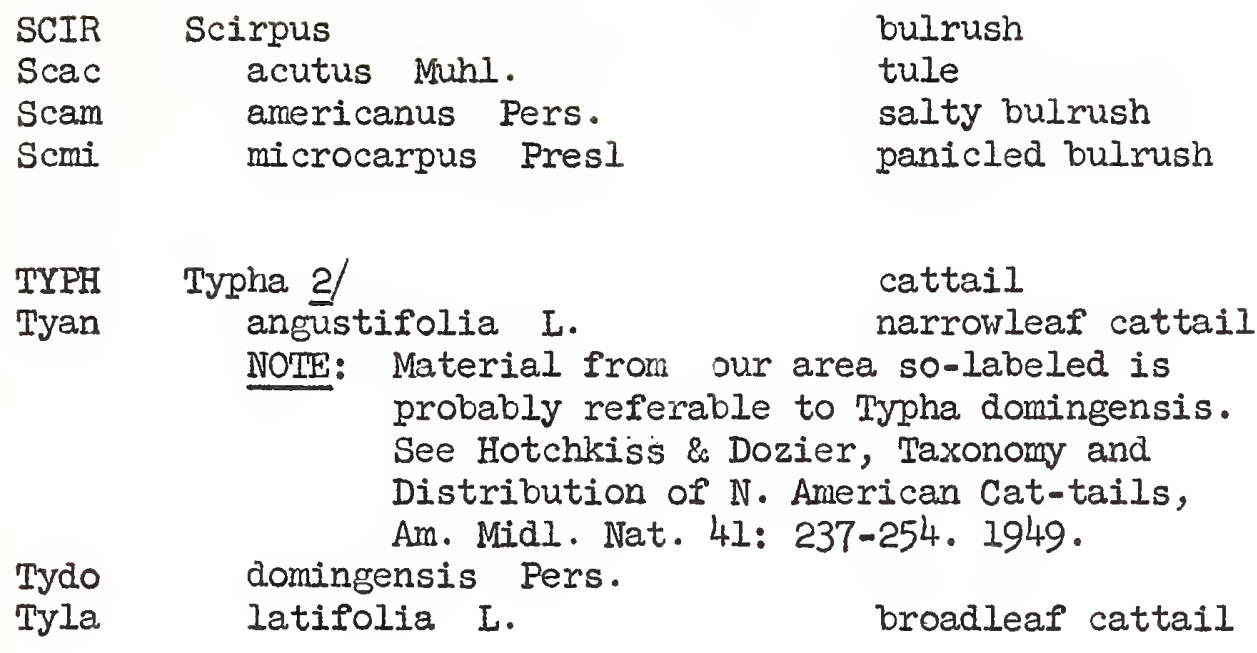

\author{
tule bulrush \\ American bulrush
}

Dominican cattail \% common cattail

2/ This genus preferably regarded as a forb.

$\%$ Suggested common name. 
FORBS IIST

$\begin{array}{lcl}\text { ABRo } & \text { Abronia } & \text { sandverbena } \\ \text { Aban } & \text { angustifolia Greene } & \text { purple sandverbena } \\ \text { Abel } & \text { elliptica A. Nels. } & \text { pink sandverbena } \\ \text { Abfr } & \text { fragrans Nutt. } & \text { snowball sandverbena } \\ \text { Abvi } & \text { villosa S. Wats. } & \text { desert sandverbena } \\ & & \text { yarrow (milfoil) } \\ \text { ACHI } & \text { Achillea } & \text { western yarrow } \\ \text { Acla } & \text { lanulosa Nutt. } & \text { monkshood } \\ & & \text { Baker monkshood } \\ \text { ACoN } & \text { Aconitum } & \text { Columbia monkshood } \\ \text { Acba } & \text { bakeri Greene } & \text { (aconite, wolfoane) } \\ \text { Acco-2 } & \text { columbianum Nutt. } & \\ & & \end{array}$

Adenostegia See Cordylanthus
ADIA Adiantum
maidenhair fern
maidenhair
AGAS Agastache
giant-hyssop
(horsemint)
giant-hyssop
Agca.
cana (Hook.) Woot. \& Standl. urticifolia Kuntze
mosquito-plant
nettleleaf giant-hyssop

Agave see under TREES AND SHRUBS

AGOS

Agar

Agau

Aggl

Aggr

Aghe
Agoseris

arizonica Greene aurantiaca (Hook.) Greene glauca (Pursh) D. Dietr. gracilens (A. Gray) Kuntze heterophylla (Nutt.) Greene

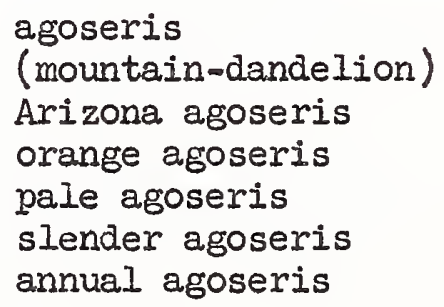

agoseris

AGRI Agrimonia

Agst striata Michx.

agrimony

roadside agrimony 
rowd and
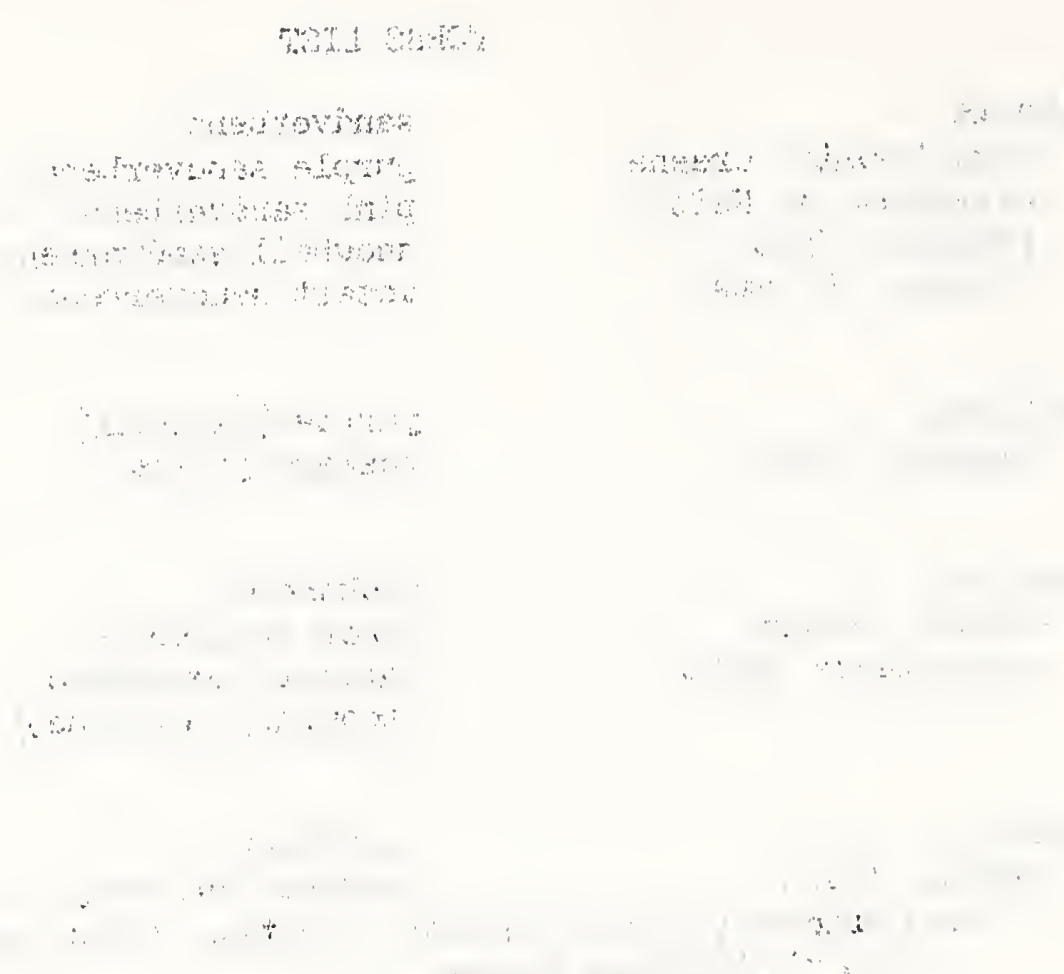

$\therefore \quad$

. 
ALIS Alisma waterplantain

Aloc Allenrolfea occidentalis ( $\mathrm{s}$. Wats.) Kuntze $\begin{aligned} & \text { iodinebush } \\ & \text { (pickleweed) }\end{aligned}$

Alin Allionia incarnata $I$.

trailing four-o'clodk

trailing allionia

(umbreliawort; wild

$$
\text { four }=0 \text { ' clock) }
$$

ALLI Allium
Alac $\quad$ acuminatum Hook.
Albr $\quad$ brevistylum S. Wats.
Alca
Alce
canadense L.

onion

tapertip onion

shortstyle onion

Canada garlic

nodding onion

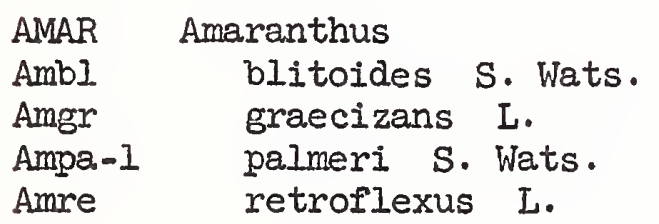

amaranth (pigweed)

prostrate amaranth

tumbleweed amaranth

carelessweed amaranth

redroot amaranth

$\begin{array}{ccc}\text { AMBR } & \text { Ambrosia } & \text { ragweed } \\ \text { Amap } & \text { aptera DC } & \text { blood ragweed } \\ \text { Amar } & \text { artemisifolia L. } & \text { common ragweed } \\ \text { Amps } & \text { psilostachya DC. } & \text { western ragweed } \\ & =\text { A. coronopifolia Torr. \& Gray }\end{array}$

AMSI Amsinckia

fiddleneck

Ampa-2 Amsonia palmeri A. Gray

Palmer amsonia

ANAC Anacharis

waterweed

ANAP Anaphalis

Anma margaritacea (I) A. Gray

Anmas

subalpina A. Gray

ANDR-2 Androsace

Anoc occidentalis Pursh

pearleverlasting

common pearleverlasting

western pearl-

everlasting

western common

pearleverlasting

rockjasmine

western rockjasmine 


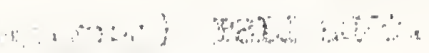

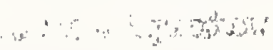

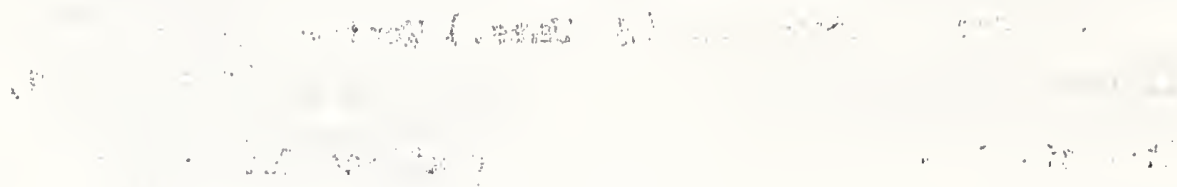


Forbs List (continued)

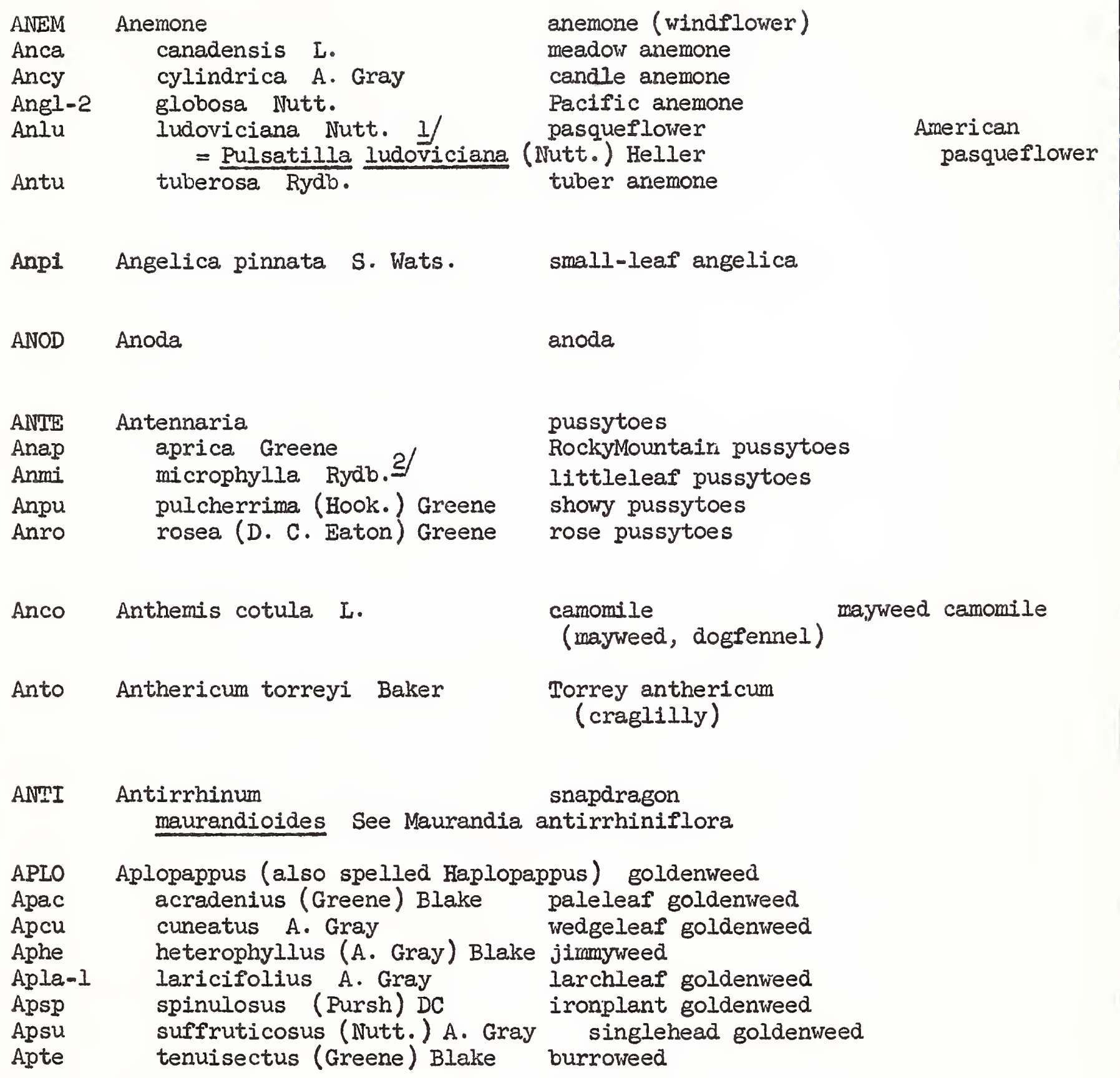


Forbs List (continued)

$\begin{array}{ll}\text { APOC } & \text { Mocynum } \\ \text { Apan } & \text { androsaemifolium I. } \\ \text { Apang } & \text { glabrum Macoun } \\ \text { Apca } & \text { cannabinum L. } \\ \text { Appu } & \text { pumilum (A. Gray) Greene } \\ \text { Apsc } & \text { scopulorum Greene }\end{array}$

Apun Apodanthera undulata A. Gray

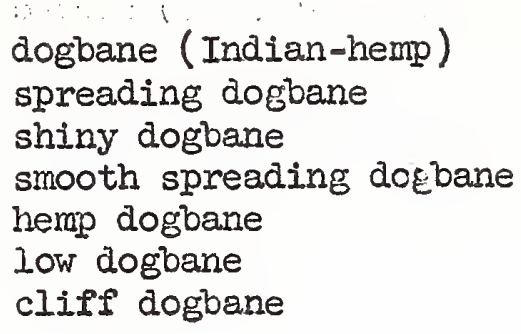

gourā ("melon-10co")

melon-gourd

NOTE: Gourd is the accepted common name for the genus Lagenaria.

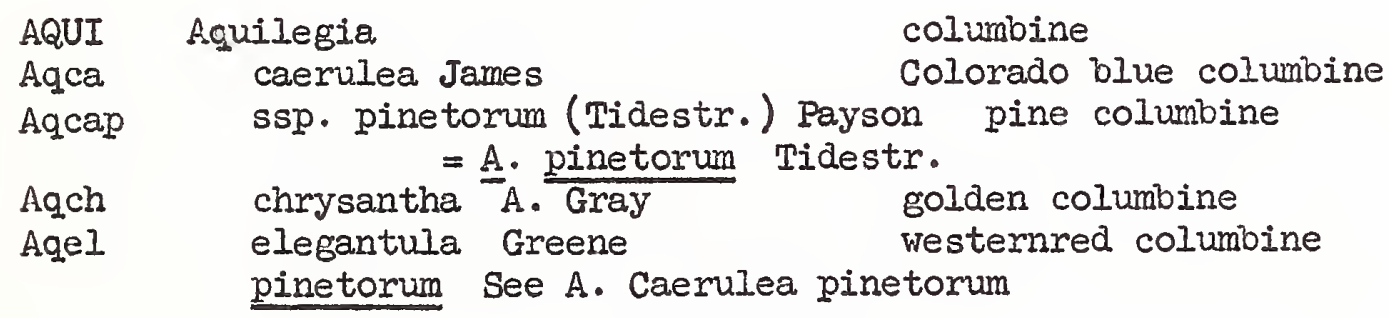

$A R A B$ Arabis

Arbr breweri s. Wats.

Arca-2 canescens Nutt.

Ardr-1 drummondii A. Gray

Argl-3 glabra (L.) Bernh.

Armi-2 microphylla Nutt.
Arnu

nuttallii B. I. Robins.

Arra.. Aralia racemosa I. rockcress

Brewer rockcress

silver rockcress

Drummond rociscress

towermustard rockcress

littleleaf rockcress

Nuttall rockcress aralia

(spikenard)

burdock

desertpoppy

sandwort

ballhead sandwort

Fendler sandwort

fescue sandwort

Siberian sandwort

Uinta sandwort

tufted sandwort
American spikenard

smaller burdock

bearpoppy 

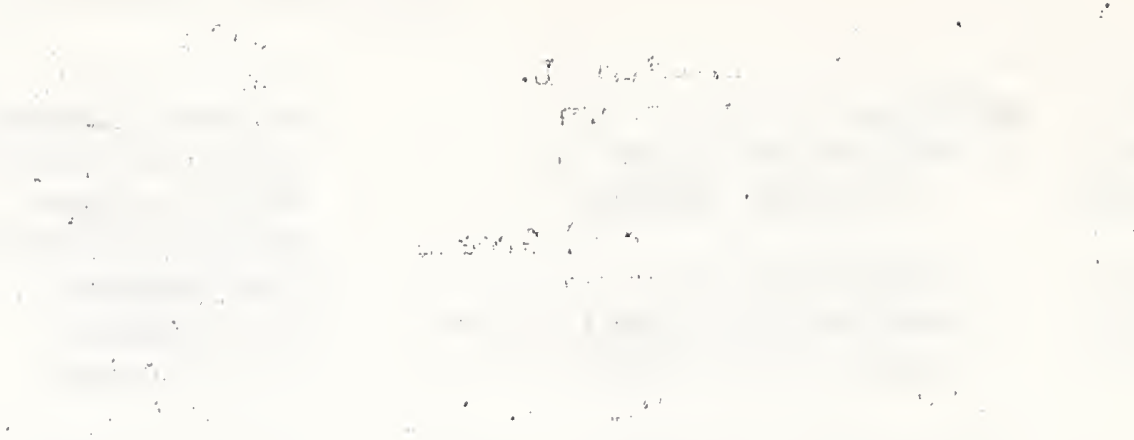
Forbs List (continueá)

$\begin{array}{lrll}\text { ARGE } & \text { Argemone } & \text { pricleiepoppy (chicalote) } \\ \text { Arpl } & \text { platyceras } & \text { Link \& otto } & \text { crested pricklepoppy } \\ \text { Arplh } & \text { hispida } & \text { (A. Gray) Prain } & \text { hedgehog pricklepoppy }\end{array}$

Argentina see Potentilla

ARIS-1 Aristolochia

Dutchmanspipe

Arwa watsonii Woot. \& Standl. Indianroot

Watson Dutchmanspipe

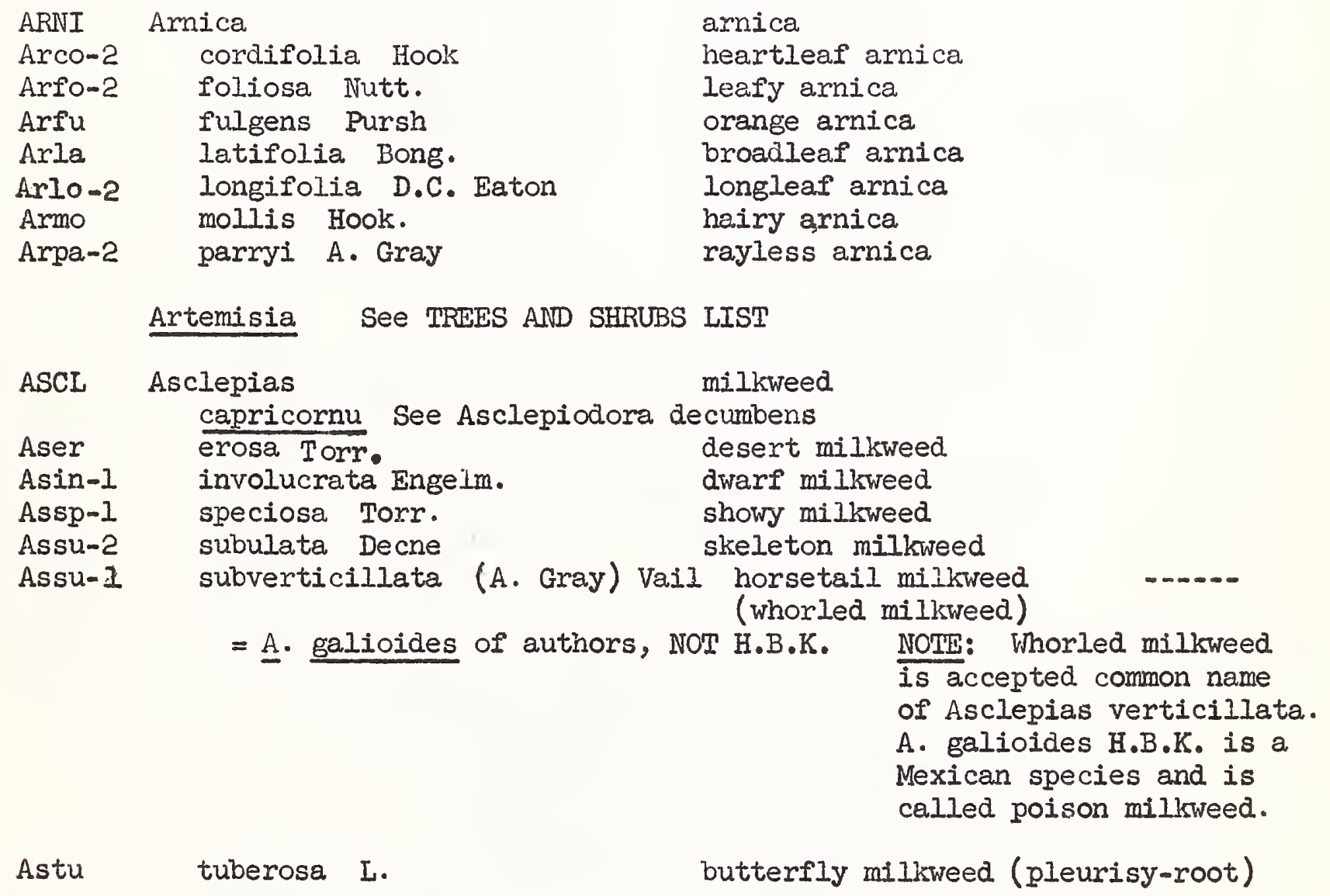

Asde Asclepiodora decumbens (Nutt.) A. Gray antelopehorn = Asclepias capricornu Woodson

spider antelopehorn

ASPL Asplenium spleenwort 


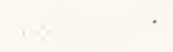


Forbs List (continued)

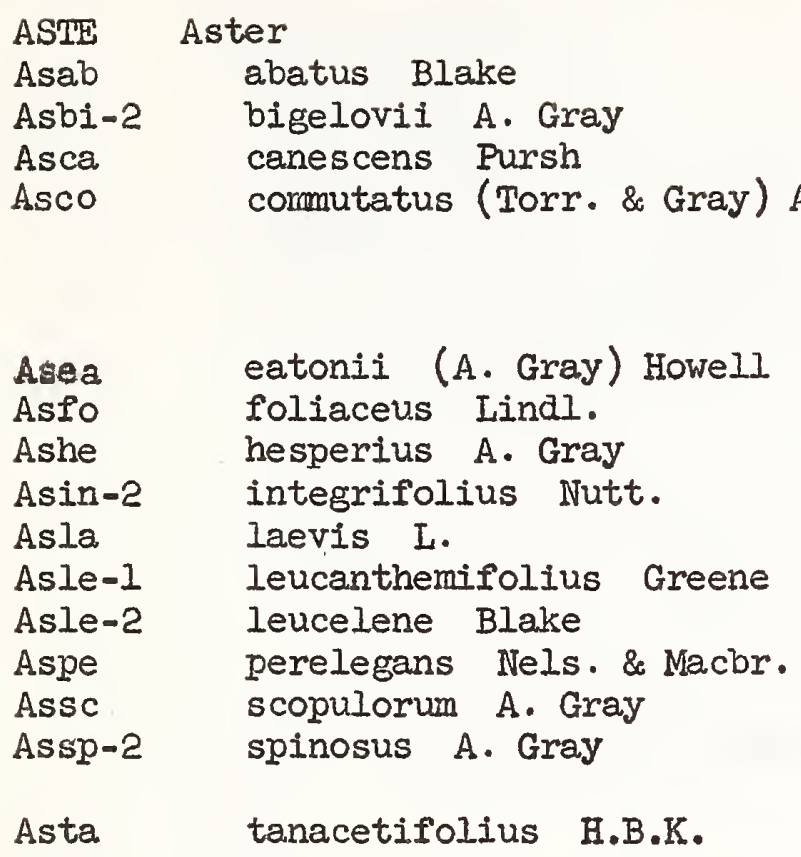

ASTR Astragalus

Asag agrestis Dougl.

Asal-1 Asal-2

Asar

Asbe

Asbi-1

Asca-1 Ascab

Ascr

Asfl

Asha

Aske

Asle -3

Aslea

Asled

Asno crassicarpus Nutt. flexuosus (Dougl.) Jones goniatus See A. agrestis haydenianus A. Gray $=$ A. araneosus Sheld. $=$ A. goniatus Nutt. allochrous A. Gray alpinus I. araneosus See Astragalus arizonicus A. Gray beckwithii Torr. \& Gray bigelovii A. Gray canadensis $I$. brevidens (Gand.) Barneby Morton Ioco

Eatons aster daisyleaf aster (devilweed) tansyleaf aster Arizona loco Beckwith milkvetch Bigelow milkvetch milkvetch-- nonpoisonous

loco -- poisonous

purple milkvetch

halfmoon $10 c 0$

alpine milkvetch leafybract aster Siskiyou aster thickstem aster smooth aster babywhite aster Nuttall aster crag aster spiny aster

devilweed aster

aster

Mohave aster

Bigelow aster

NOTE: Showy aster is the accepted common name for Aster conspicuus.

\section{= A. mortonii of authors, NOT Nutt.}

NOTW: Astragalus mortonii Nutt. is primarily a northwestern species, apparently not in our Intermountain or Southwestern areas; material so called is, fide Dr. Barneby, A. canadensis var. brevidens. groundplum milkvetch flexible milkvetch Canada milkvetch

Hayden poisonvetch impensus See A. kentrophyta var. elatus kentrophyta var. elatus S. Wats. Thistle milkvetch = A. impensus (Sheld.) Woot. \& Standl. lentiginosus Dougl. specklepod $10 c 0$ araneosus (Sheld.) Bameby dryplains milkvetch diphysus (A. Gray) Jones blue loco (rattlepod) mortoni see A. lentiginosus var, araneosus nothoxys A. Gray sheep loco 


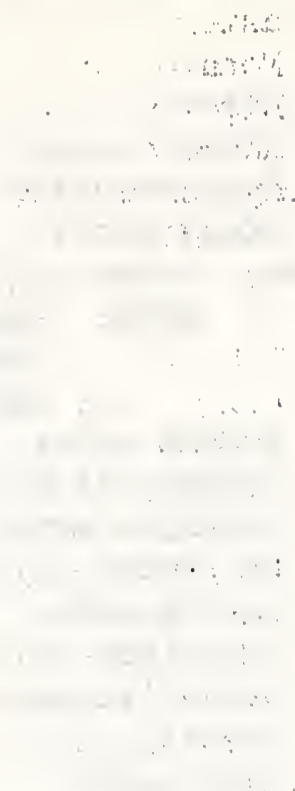

an1 1

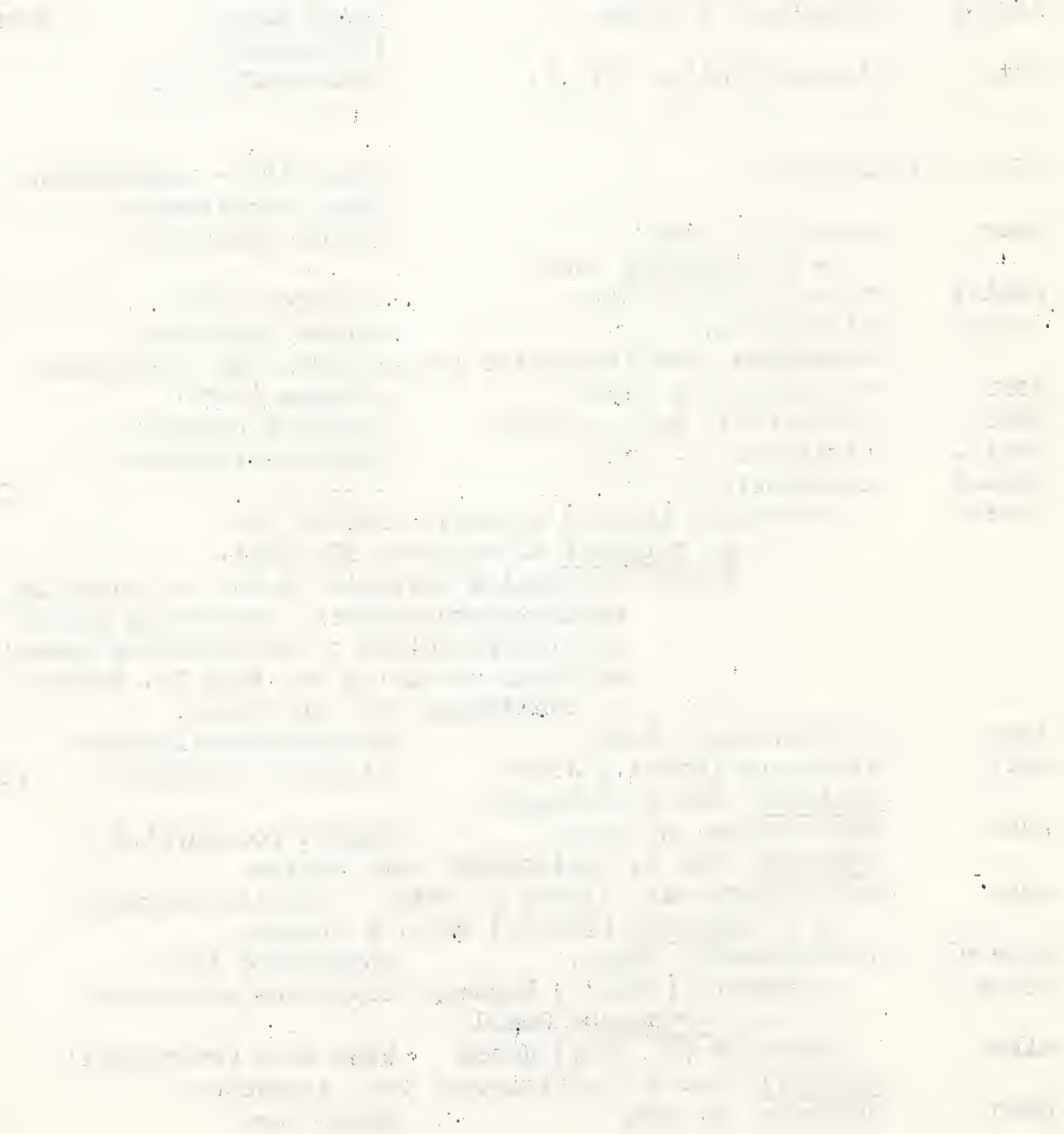


Forbs List (continued)

\begin{tabular}{|c|c|c|}
\hline$\because$ & Astragalus (continued) & \\
\hline Asnu & nuttallianus DC. & Nuttall loco \\
\hline Aspa & pattersonii A. Gray & Patterson loco \\
\hline Aspu & purshii Dougl. & Pursh $10 c 0$ \\
\hline & remulcus See A. tephrodes var & - brachylobus \\
\hline Aste & 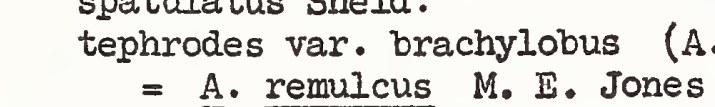 & - Gray) Barneby towline loco \\
\hline $\begin{array}{l}\text { Asth } \\
\text { Aswo }\end{array}$ & $\begin{array}{l}\text { thurberi } \overline{\text { A. Gray }} \\
\text { wootonii Sheld. }\end{array}$ & $\begin{array}{l}\text { Thurber loco } \\
\text { Wooton loco }\end{array}$ \\
\hline Atfi & Athyrium filix-femina & ladyfern \\
\hline & & $\begin{array}{l}\text { NOTE: The common name of ladyfern } \\
\text { applicable to this species } \\
\text { rather than to the genus. }\end{array}$ \\
\hline
\end{tabular}

Atriplex See TREES AND SHRUBS IIST

\begin{tabular}{|c|c|c|}
\hline $\begin{array}{l}\text { BAER } \\
\text { Bach }\end{array}$ & $\begin{array}{l}\text { Baeria } \\
\text { chrysostoma Fisch. \& Mey. }\end{array}$ & $\begin{array}{l}\text { goldfields } \\
\text { goldfields }\end{array}$ \\
\hline BAHI & Bahia & $\begin{array}{l}\text { bahia (ragweed) } \\
\text { NOTE: Ragwia } \\
\text { common name for Ambrosia. }\end{array}$ \\
\hline $\begin{array}{l}\text { Badi } \\
\text { Bane } \\
\text { Baop } \\
\text { Bawo }\end{array}$ & $\begin{array}{l}\text { dissecta (A. Gray) Britton } \\
\text { neomexicana A. Gray } \\
\text { oppositifolia (Nutt.) DC. } \\
\text { woodhousei A. Gray }\end{array}$ & $\begin{array}{l}\text { ragleaf bahia } \\
\text { New-Mexican bahia } \\
\text { plains bahia } \\
\text { Woodhouse bahia }\end{array}$ \\
\hline $\begin{array}{l}\text { BAIL } \\
\text { Bamu }\end{array}$ & $\begin{array}{l}\text { Baileya } \\
\text { multiradiata Harv. \& A. Gray }\end{array}$ & $\begin{array}{l}\text { baileya } \\
\text { (desertmarigold) } \\
\text { desert baileya. }\end{array}$ \\
\hline $\begin{array}{l}\text { BAIS } \\
\text { Basa-2 }\end{array}$ & $\begin{array}{l}\text { Balsamorhiza } \\
\text { sagittata (Pursh) Nutt. }\end{array}$ & $\begin{array}{l}\text { balsamroot } \\
\text { arrowleaf balsamroot }\end{array}$ \\
\hline
\end{tabular}

Baor Barbarea orthoceras Ledeb. wintercress erectpod wintercress

Bassia hyssopifolia See Echinopsilon hyssopifolium

Bete Bergia texana (Hook.) Seubert Texas bergia

Beer Bervla erecta (Huds.) Coville waterparsnip stalky berula

NOTE: Waterparsnip is the accepted common name for Sium. 


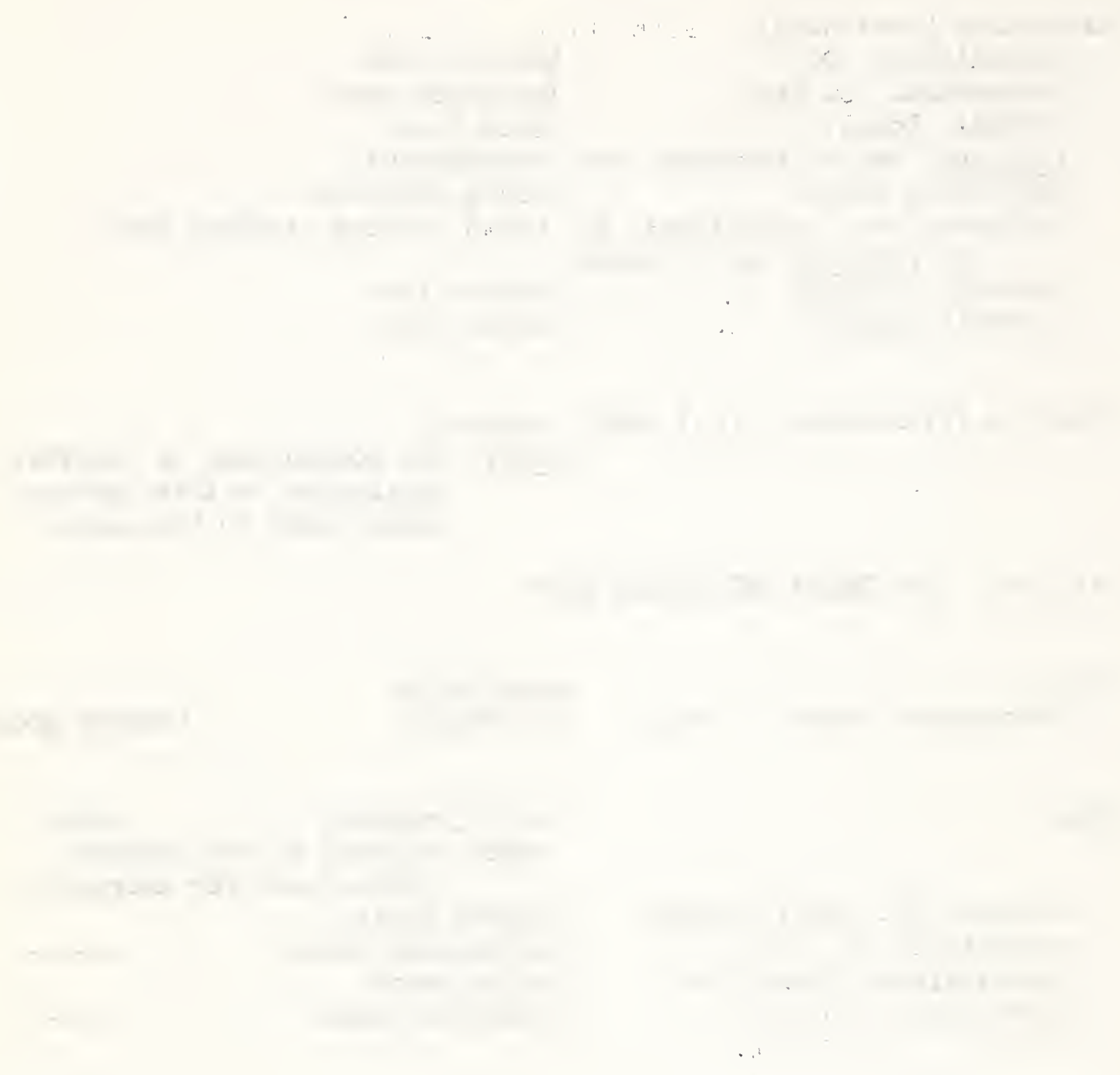


Forbs List (continued)

\section{BIDE Bidens \\ Bibi bigelovii A. Gray \\ Bihe heterosperma A. Gray \\ Bila laevis (I.) B.S.P. \\ Bile leptocephala Sherff \\ Bite tenuisecta A. Gray \\ Blitum See Chenopodium}

beggarticks

water beggarticks

bur-marigold

smooth beggarticks

pitchfork beggarticks

sticktight beggarticks

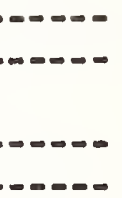

scarlet spiderling

spiderling

trailing spiderling

Coulter spiderling

erect spiderling

grapefern

mustard

rocketsalad

(roquette)

white mustard

Indian mustard

India mustard

NOTE: This species comes from India

charlock

black mustard

BrickeIlia see TREES AND SHRUBS IIST

Brodiaea See Dichelostema

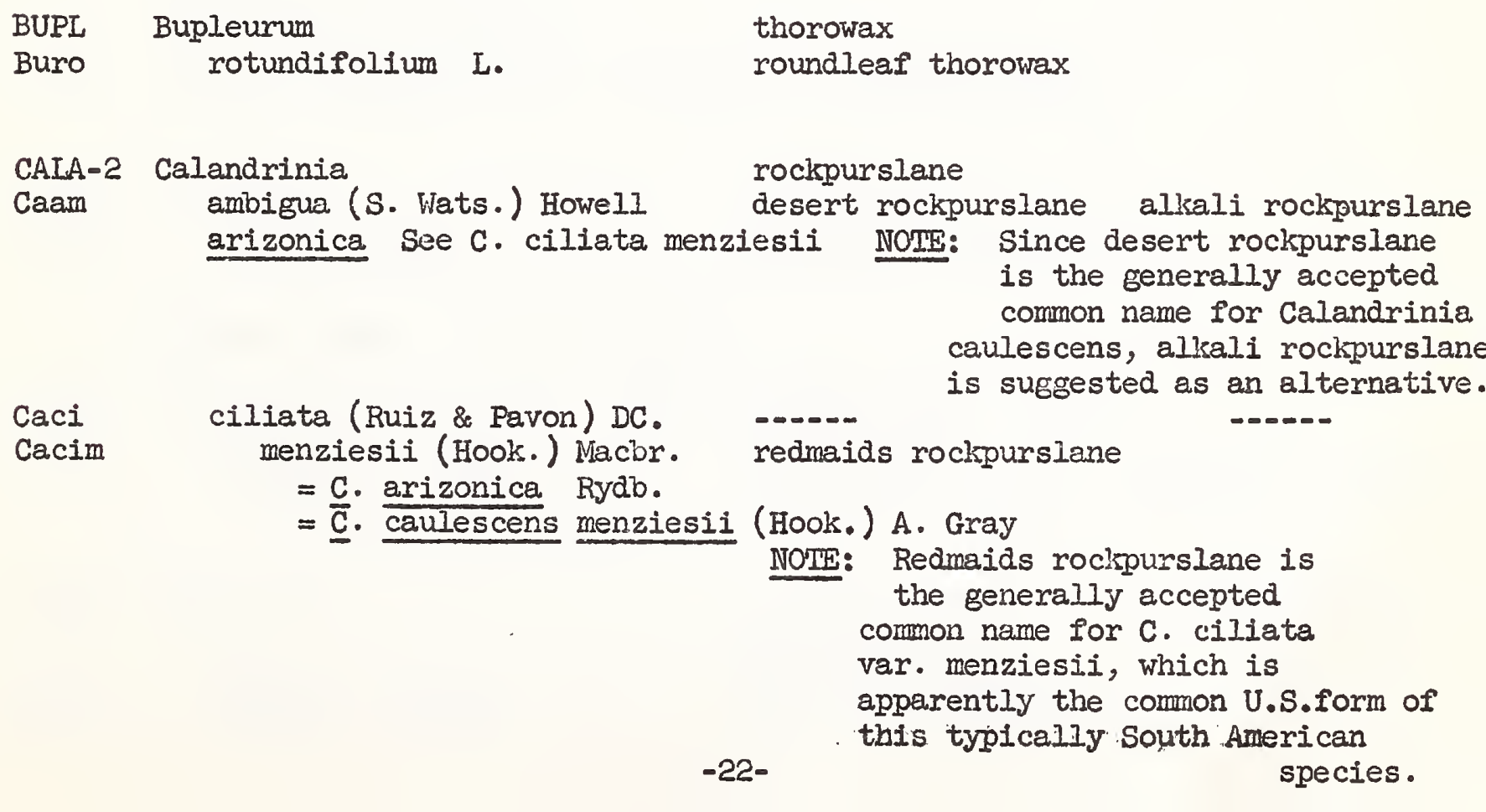




\section{.}

$x_{1}=$

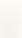

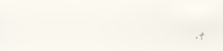




$$
\begin{aligned}
& \text { CALL-I Callirhoë = Malva, in part } \\
& \begin{aligned}
& \text { Cale-3 leiocarpa R.F. Martin } \\
&=\text { C. pedata (Torr. \& Gray) A. Gray } \\
&=\text { Malva pedata Torr. \& Gray } \\
&=\text { "Nuttallia pedata" of A. Gray, NOT Nutt. }
\end{aligned}
\end{aligned}
$$

\begin{tabular}{|c|c|c|c|}
\hline CALO & Calochortus & mariposa & \\
\hline Cagu & gunnisonii s. Wats. & Gunnison mariposa & \\
\hline Canu & nuttallii Torr. \& Gray & sego-lily & $\begin{array}{l}\text { sego-lily } \\
\text { mariposa }\end{array}$ \\
\hline Canua & aureus (s. Wats.) Ownbey & golden sego-lily & $\begin{array}{c}\text { golden sego-lily } \\
\text { mariposa }\end{array}$ \\
\hline Cale-I & Caltha leptosepala DC. & $\begin{array}{l}\text { marshmarigold } \\
\text { (elkslip) }\end{array}$ & $\begin{array}{l}\text { elkslip marsh- } \\
\text { marigold }\end{array}$ \\
\hline $\begin{array}{l}\text { CAME } \\
\text { Cami-2 } \\
\text { Casa }\end{array}$ & $\begin{array}{l}\text { Camelina } \\
\text { microcarpa Andrz. } \\
\text { sativa (I.) Crantz }\end{array}$ & $\begin{array}{l}\text { falseflax } \\
\text { littlepod falseflax } \\
\text { bigseed falseflax }\end{array}$ & \\
\hline $\begin{array}{l}\text { CAMP } \\
\text { Capa } \\
\text { Cape } \\
\text { Caro-I }\end{array}$ & $\begin{array}{l}\text { Campanula } \\
\text { parryi A. Gray } \\
\text { petiolata A. DC. } \\
\text { rotundifolia I. }\end{array}$ & $\begin{array}{l}\text { bellflower } \\
\text { Parry bellflower } \\
\text { harebell } \\
\text { bluebell }\end{array}$ & American harebell \\
\hline & $\begin{array}{l}\text { Campe See Barbarea } \\
\text { Capnoides See Corydalis }\end{array}$ & & \\
\hline
\end{tabular}

Callisteris collina see Gilia greeneana

\begin{tabular}{|c|c|c|c|}
\hline $\begin{array}{l}\text { CASS } \\
\text { Caco-1 } \\
\text { Cawr }\end{array}$ & $\begin{array}{l}\text { Cassia See also under TREES } \\
\text { covesii A. Gray } \\
\text { wrightii A. Gray } \\
\text { = Chamaecrista wrightii (A }\end{array}$ & $\begin{array}{l}\text { AND SHRUBS IIST senna } \\
\text { hairy senna } \\
\text { partridge-pea } \\
\text { A. Gray) Woot. \& StandI. }\end{array}$ & $\begin{array}{c}\text { Wrights partridge- } \\
\text { pea }\end{array}$ \\
\hline CAST & Castilleja & $\begin{array}{l}\text { paintbrush } \\
\text { (painted-cup) }\end{array}$ & painted-cup \\
\hline $\begin{array}{l}\text { Cafl } \\
\text { Cain-2 }\end{array}$ & $\begin{array}{l}\text { flava S. Wats. } \\
\text { integra A. Gray }\end{array}$ & $\begin{array}{l}\text { yellow paintbrush } \\
\text { wholeleaf paintbrush }\end{array}$ & \\
\hline
\end{tabular}

Cabu Capsella bursa-pastoris (I.) Medic. shepherds-purse

$\begin{array}{lll}\text { CARD } & \text { Cardamine } & \text { bittercress } \\ \text { Caco-2 } & \text { cordifolia A. Gray } & \text { heartleaf bittercress } \\ \text { Cahi } & \text { hirsuta Muhl. } & \text { Pennsylvania bittercress }\end{array}$

Cardaria draba see Lepidium draba. 

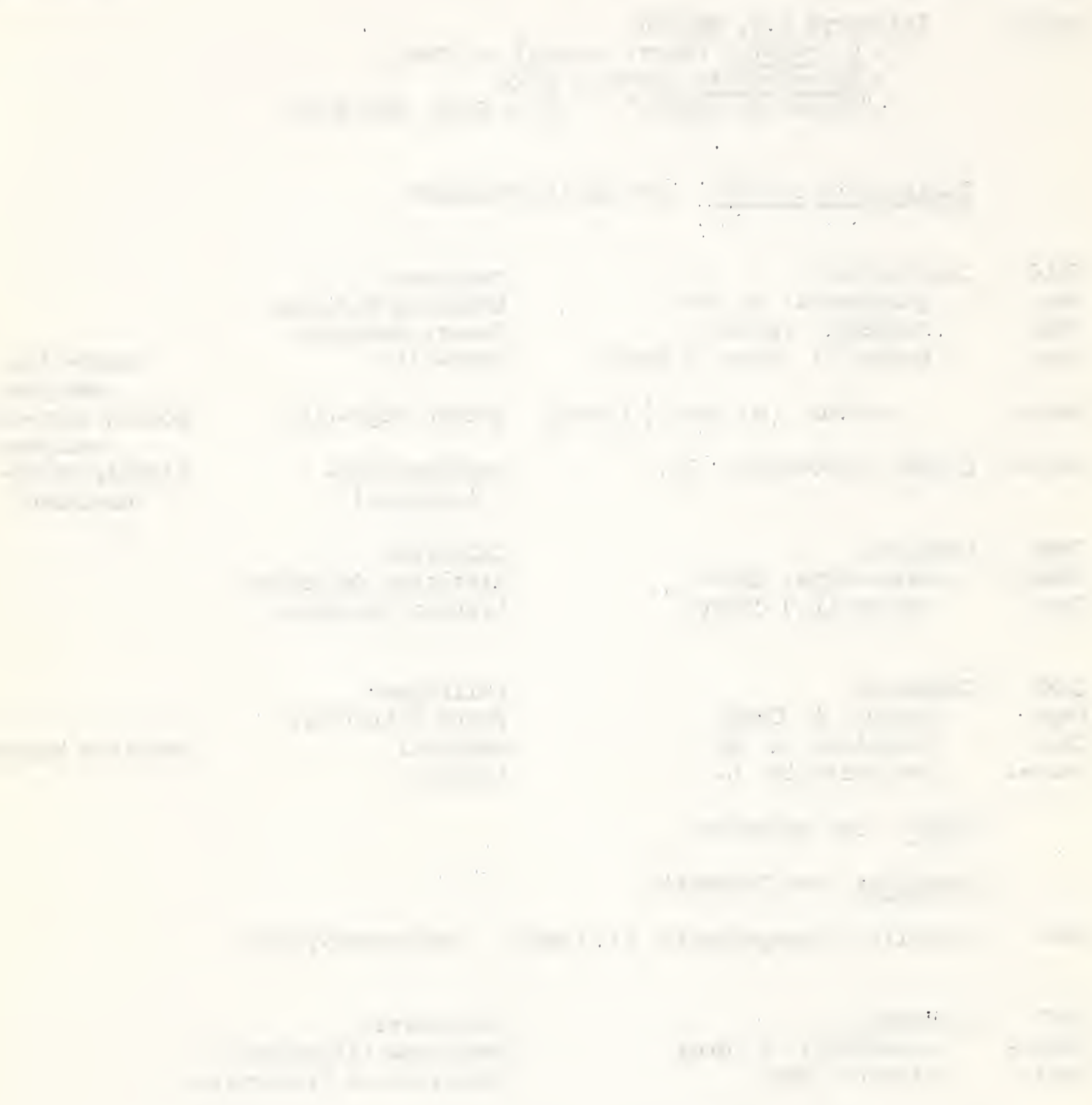
Forbs List (continued)

\begin{tabular}{|c|c|c|c|}
\hline & Castilleja (continued) & & \\
\hline Cali-1 & linariaefolia Benth. & Wyoming paintbrush & \\
\hline Calu & luteovirens Rydb. & yellowgreen paintbrus & \\
\hline Cami & miniata Dougl. & scarlet paintbrush & \\
\hline Carh & rhexifolia Rydb. & splitleaf paintbrush & \\
\hline Case & $\begin{array}{l}\text { septentrionalis Lindl. } \\
\text { = C. sulphurea Rydb. }\end{array}$ & sulphur paintbrush & Sulfur painted-cup \\
\hline$\vdots$ & & & \\
\hline Cami-1 & Caucalis microcarpa Hook \& Arn. & false-carrot & \\
\hline CENT-1 & Centaurea & centaurea & \\
\hline Ceam & americana Nutt. & basketflower & $\begin{array}{l}\text { basketflower } \\
\text { centaurea }\end{array}$ \\
\hline Ceca & calcitrapa & star-thistle & -..... \\
\hline Cecy & cyanus L. & cornflower (bachelor' & s button) \\
\hline $\begin{array}{l}\text { Ceme } \\
\therefore \because\end{array}$ & $\begin{array}{l}\text { melitensis } L \text {. } \\
\text { picris see C. repens. }\end{array}$ & Malta centaurea & Malta star-thistle \\
\hline Cere-1 & $\begin{array}{l}\text { repens } \\
=C \text {. picris Pall. }\end{array}$ & Russian knapweed & Russian centaurea \\
\hline Ceso & solstítialis L. & yellow centaurea & \\
\hline CENT-2 & Centaurium & canchalagua & centaurium \\
\hline $\begin{array}{l}\text { CERA } \\
\text { Cear }\end{array}$ & $\begin{array}{l}\text { Cerastium } \\
\text { arvense }\end{array}$ & $\begin{array}{l}\text { mouse-ear } \\
\text { starmy mouse-ear }\end{array}$ & $\begin{array}{c}\text { cerastium } \\
\text { starry cerastium }\end{array}$ \\
\hline Cebe-2 & beeringianum Cham. \& Schlecht & - alpine mouse-ear & (n) \\
\hline Cenu & nutans Raf. & powderhorn mouse-ear & ---- \\
\hline CHAE & Chaenactis & false-yarrow & $\begin{array}{c}\text { Chaenactis } \\
\text { (False-yarrow) }\end{array}$ \\
\hline $\begin{array}{l}\text { Chdo } \\
\text { Chst }\end{array}$ & $\begin{array}{l}\text { douglasii (Hook.) Hook. \& Am. } \\
\text { stevioides Hook. \& Arn. }\end{array}$ & $\begin{array}{l}\text { Douglas false-yarrow } \\
\text { dryland false-yarrow }\end{array}$ & Douglas chaenactis \\
\hline & Chamaecrista See Cassia & & \\
\hline CHEI & Cheilanthes & lipfern & \\
\hline
\end{tabular}

Cheirinia See Erysimum

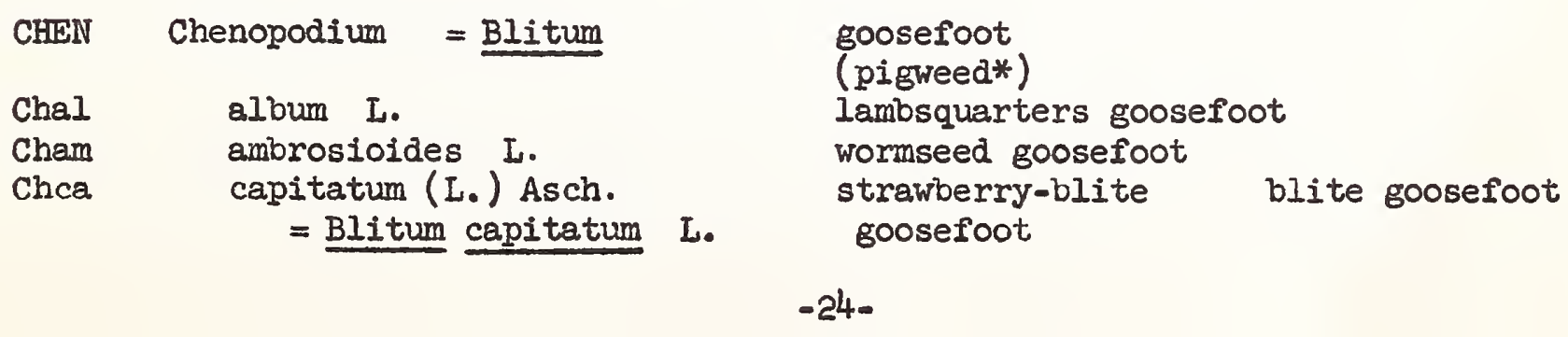




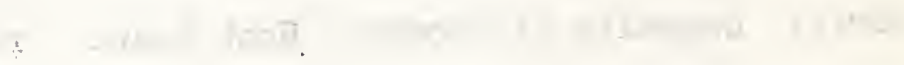

I.

in in

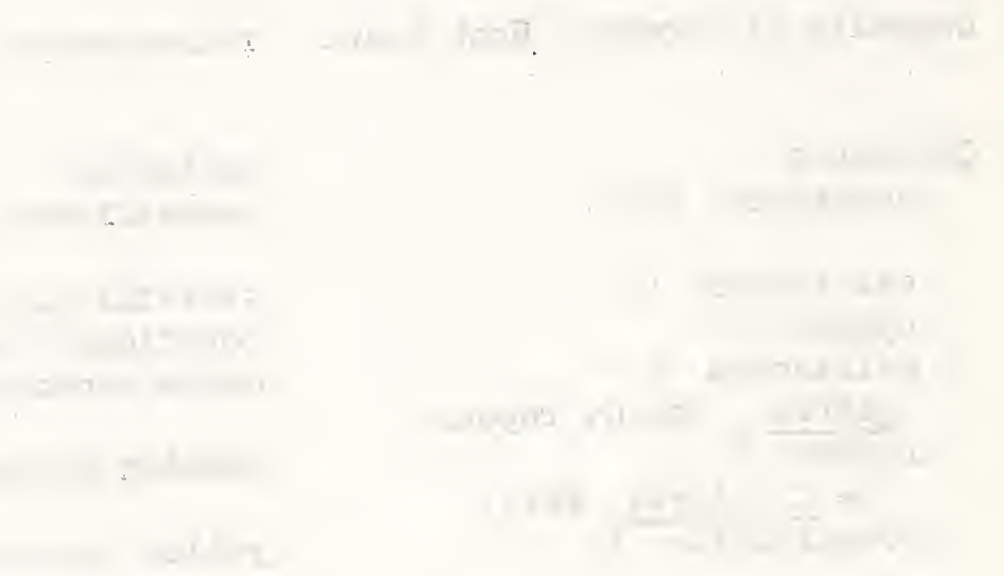

$$
\begin{aligned}
& \text { ‥ i } \\
& 1+-1 .+2=
\end{aligned}
$$

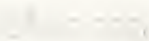

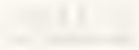

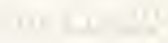$$
\text { 2. }
$$ 


$\begin{array}{lc} & \text { Chenopodium (continued) } \\ \text { Chfr } & \text { fremontii S. Wats. } \\ \text { Chin } & \text { incisum Poir. } \\ \text { Chle } & \text { leptophyllum Nutt } \\ \text { Chmu } & \text { murale L. } \\ \text { Chpa-l } & \text { paganum Reichenb. }\end{array}$

CHIM Chimaphila

Chma maculata (I.) Pursh

Chum umbellata (I.) Nutt.

Chorizanthe
Fremont goosefoot

ragleaf goosefoot

slimleaf goosefoot

nettleleaf goosefoot

pigweed goosefoot

pipsissewa

rock pipsissewa

princespine pipsissewa

Turksrug

NOTE: Turksrug is accepted common name for Chorizanthe staticoides.

\section{CHRY-2 Chrysopsis \\ Chfo \\ Chhi \\ Chvi-2 foliosa hispida (Hook) DC. villosa (Pursh) Nutt.}

goldaster leafy goldaster rough goldaster hairy goldaster

Ciin Cichorium intybus $I$.

chicory common chicory

Cido Cicuta douglasii (DC.) Coult. \& Rose waterhemlock (wild parsnip*, cowbane)

CIRC Circaea

Cipa pacifica Asch. \& Magn. NOTE: Doubtfully distinct from alpine circaea (c. alpina). enchanters nightshade circaea

(enchanters-nightshade)
CIRS

Ciar

Cidr

Cigr

Cila-1

Cine

Ciro

Ciun
Cirsium

arizonicum (A. Gray) Petrak drummondii Torr. \& Gray grahamii A. Gray lanceolatum (I.) Hill neomexicanum A. Gray rothrockii (A. Gray) Petrak undulatum (Nutt.) Spreng. thistle

Arizona thistle creamy thistle deep-purple thistle bull thistle lavender thistle carmine thistle wavyleaf thistle

Citr Cissus trifoliata L.

treebine

Clrh Clarkia rhomboidea Dougl.

Drummond thistle
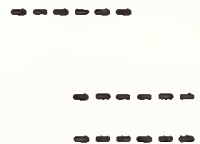


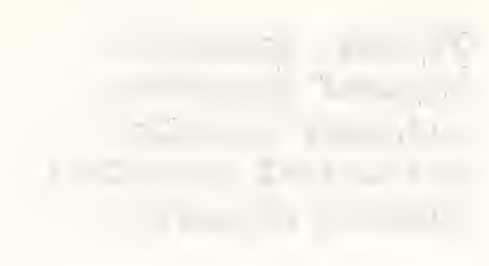

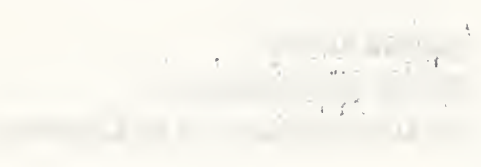

$$
\therefore \because
$$

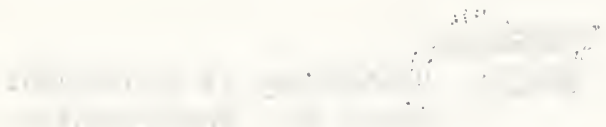

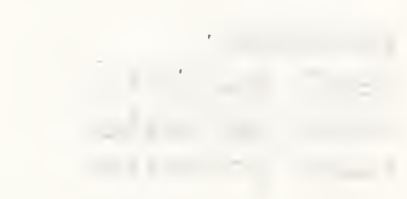

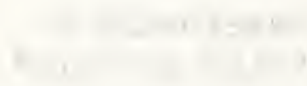

1.

$$
+
$$

x.s...

$$
1+1-1
$$

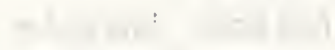

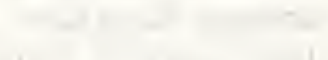

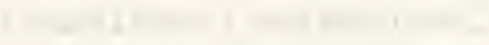

nt
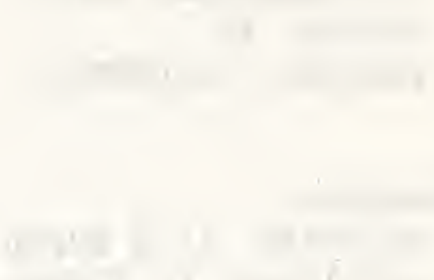

$-\frac{1}{1}+1$
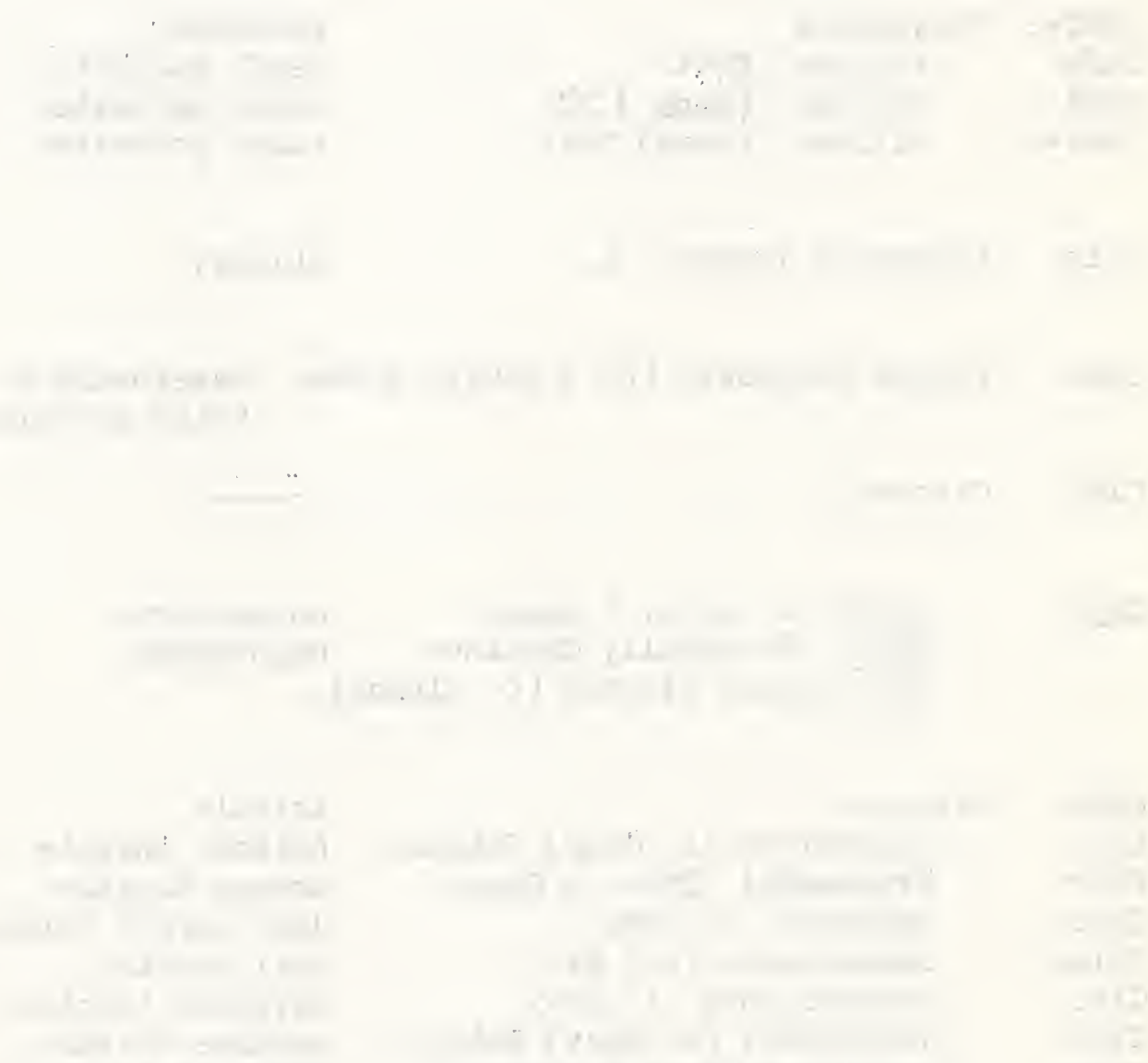

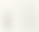

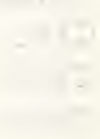

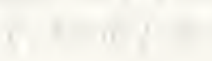




$$
\text { Forbs List (continued) }
$$

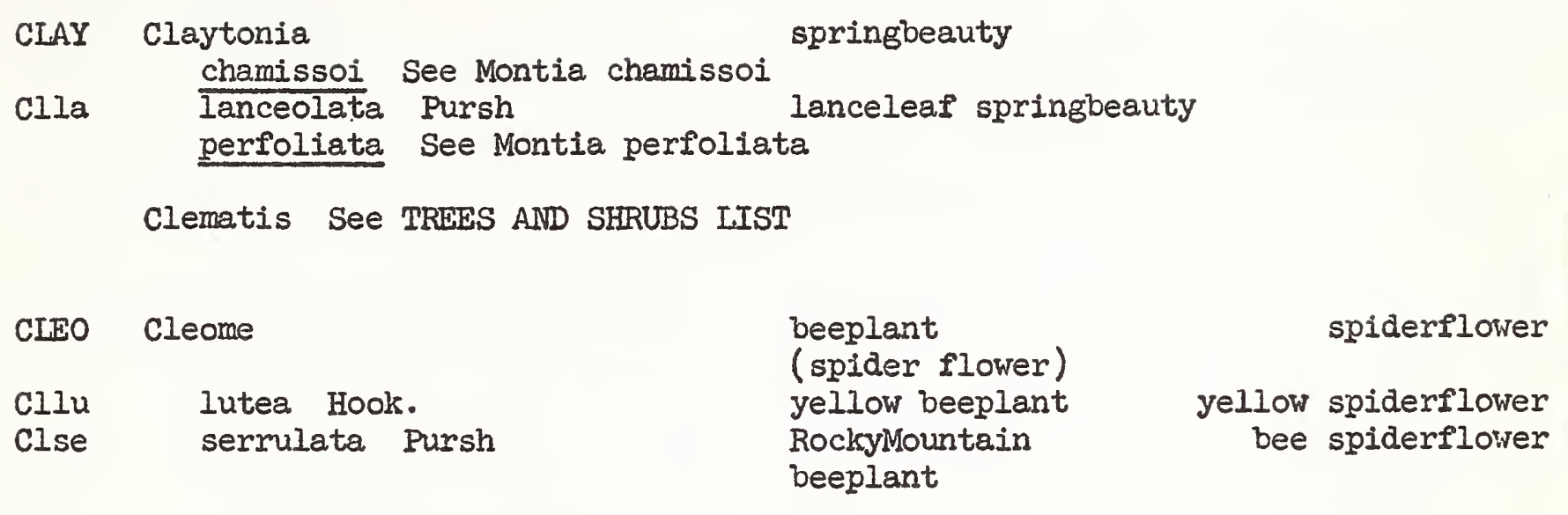

Clob Cleomella obtusifolia Torr. \& Frem. Mohave stinkweed

Clma Clitoria mariana L. butterfly-pea

Atlantic pigeonwings

Coldenia See TREES AND SHRUBS LIST

Coleosanthus See Brickellia under TREES AND SHRUBS IIST

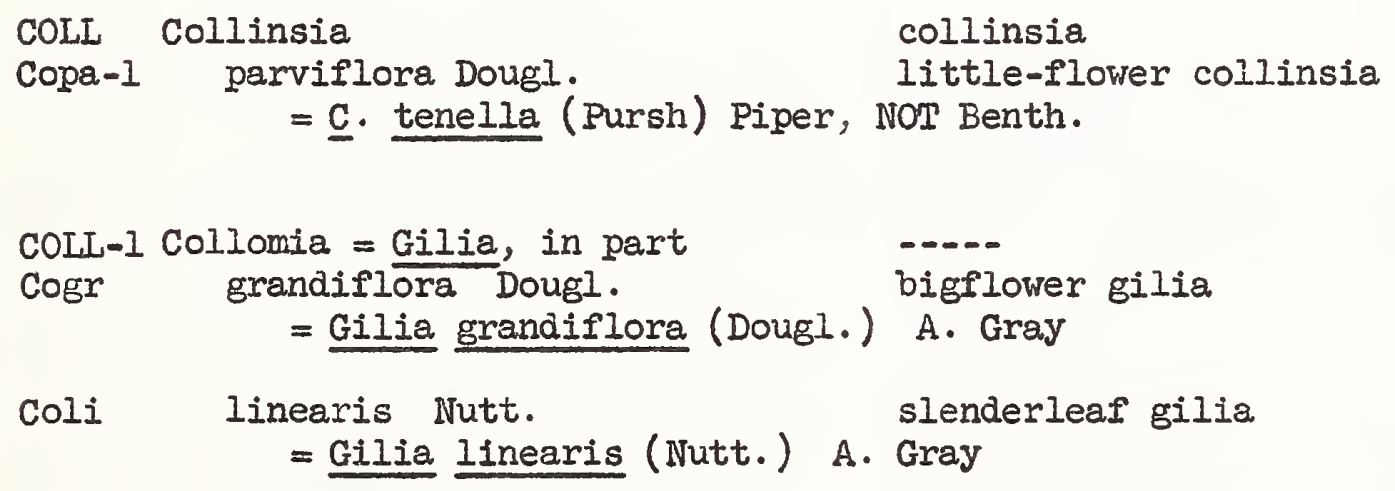

COLL-1 Collomia = Gilia, in part

Cogr grandiflora Dougl. = Gilia grandiflora (Dougl.) A. Gray

Coli linearis Nutt. = Gilia linearis (Nutt.) A. Gray

collomia \% bigflower collomia \%

slenderleaf collomia \%
Copa-2 Comandra pallida A. DC. bastard toadflax common comandra. = $\underline{\text { C. umbellata }}$ (L.) Nutt. NOTE: Toadflax is accepted conmon name for Iinaria.
COMM Commelina crispa see C. erecta var. crispa
Codi dianthifolia Delile birdbill dayflower
Coer erecta L. erect dayflower
Coerc crispa (Woot.) Palmer \& Steyerm. curlyleaf dayflower $=\underline{\mathrm{C}}$. crispa Woot.




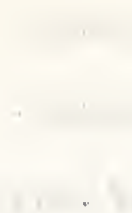

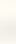

, :

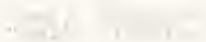

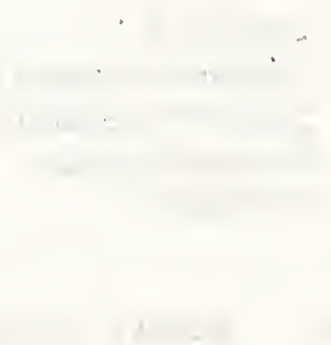

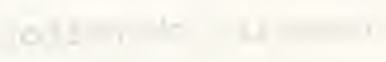

$x^{2}+n^{2}$
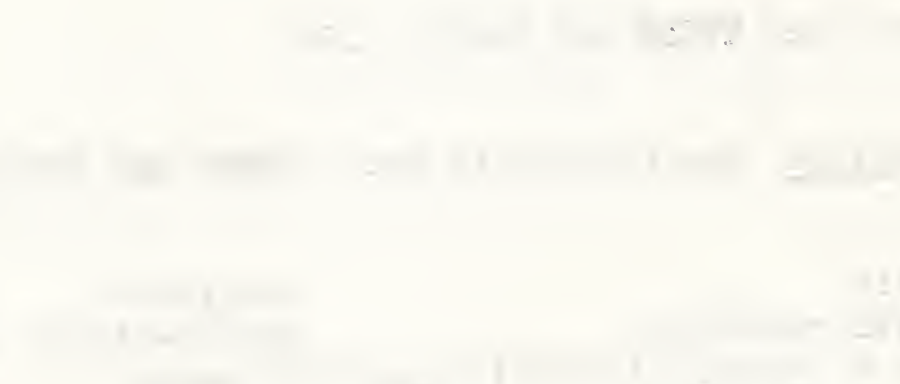

(1)

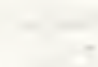

(n)

$-x^{-1}$

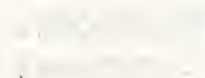




\section{Forbs List (continued)}

\begin{tabular}{|c|c|c|c|}
\hline CONI & Conioselinum & hemlockparsley & \\
\hline $\operatorname{cose}$ & scopulorum (A. Gray) coult. & \& Rose hemlockparsley & $\begin{array}{l}\text { RockyMountain } \\
\text { hemlockparsley } \%\end{array}$ \\
\hline Coma-1 & Conium maculatum $\mathrm{L}$. & poisonhemlock & \\
\hline Come -3 & Conopholis mexicana A. Gray & squawroot & 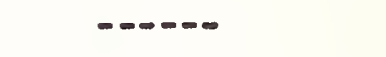 \\
\hline CONV & Convolvulus & $\begin{array}{l}\text { bindweed } \\
\text { (glorybind) }\end{array}$ & glorybind \\
\hline Coar-1 & arvensis $\mathrm{I}$. & field bindweed & $\begin{array}{l}\text { European } \\
\text { glorybind }\end{array}$ \\
\hline $\operatorname{coln}$ & incanus $\operatorname{Vahl}$ & climbing bindweed & $\begin{array}{l}\text { Nebraska } \\
\text { glorybind }\end{array}$ \\
\hline Cose & sepium I. & hedge bindweed & hedge giorybind \\
\hline CONY & $\begin{array}{l}\text { Conyza } \\
\text { NOTE: Conyza is conserved }\end{array}$ & $\begin{array}{l}\text { rayless-aster } \\
\text { against Eschenbachia. }\end{array}$ & \\
\hline $\begin{array}{l}\text { CORA } \\
\text { Coma-2 } \\
\text { Cost-2 } \\
\text { Cowi }\end{array}$ & $\begin{array}{l}\text { Corallorrhiza } \\
\text { maculata Raf. } \\
\text { striata Lindl. } \\
\text { wisteriana Conrad }\end{array}$ & $\begin{array}{l}\text { coralroot } \\
\text { spotted coralroot } \\
\text { hooded coralroot } \\
\text { Wister coralroot }\end{array}$ & \\
\hline CORD & Cordylanthus $=$ Adenostegia & $\begin{array}{l}\text { birdbeak } \\
\text { (clubflower) }\end{array}$ & birdbeak \\
\hline $\begin{array}{l}\text { Cora-2 } \\
\text { Cowr }\end{array}$ & $\begin{array}{l}\text { ramosus Nutt. } \\
\text { wrightii A. Gray }\end{array}$ & $\begin{array}{l}\text { brushy clubflower } \\
\text { plumeweed clubflower }\end{array}$ & $\begin{array}{l}\text { bushy birdbeak } \\
\text { Wright birdbeak }\end{array}$ \\
\hline $\begin{array}{l}\text { CORE } \\
\text { COca-1 }\end{array}$ & $\begin{array}{l}\text { Coreopsis } \\
\text { cardaminefolia (DC.) Torr. }\end{array}$ & $\begin{array}{l}\text { tickseed } \\
\text { \& Gray cardamine tickseed }\end{array}$ & $\begin{array}{l}\text { coreopsis } \\
\text { cardamine } \\
\text { coreopsis }\end{array}$ \\
\hline $\begin{array}{l}\text { Codo } \\
\text { Coti }\end{array}$ & $\begin{array}{l}\text { douglasii (DC.) H.M.Hall } \\
\text { tinctoria Nutt. }\end{array}$ & $\begin{array}{l}\text { Douglas tickseed } \\
\text { plains tickseed } \\
\text { NOTE: Tickseed is accep } \\
\text { name for Corisper }\end{array}$ & $\begin{array}{l}\text { Douglas coreopsis } \\
\text { plains coreopsis } \\
\text { pted common } \\
\text { mum. }\end{array}$ \\
\hline $\begin{array}{l}\text { CORI } \\
\text { Cohy }\end{array}$ & $\begin{array}{l}\text { Corispermum } \\
\text { hyssopifolium L. }\end{array}$ & $\begin{array}{l}\text { bugseed } \\
\text { hyssopleaf bugseed }\end{array}$ & $\begin{array}{l}\text { tickseed } \\
\text { hyssopleaf } \\
\text { tickseed }\end{array}$ \\
\hline Coni & nitidum Kit. & nits bugseed & \\
\hline
\end{tabular}

\% Suggested common name. 


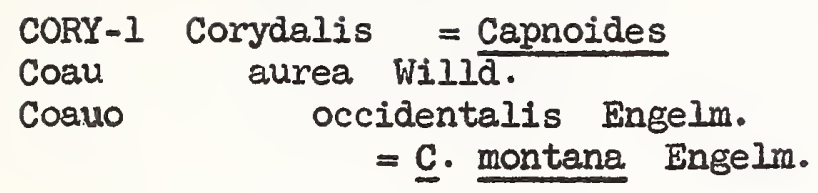

CosM Cosmos

$\begin{array}{ll}\text { CREP } & \text { Crepis } \\ \text { Crac } & \text { acuminata Nutt. } \\ \text { Crmo } & \text { modocensis Greene } \\ & =\text { C. Scopulorum Coville } \\ \text { Croc } & \text { occidentalis Nutt. } \\ \text { Crru } & \text { runcinata Torr. \& Gray } \\ & \text { scopulorum See C. modocensis }\end{array}$

CROT-1 Crotalaria

Crpu pumila ortega

Crsa sagittalis L. corydalis

golden corydalis

mountain corydalis

cosmos

hawksbeard

tapertip hawksbeard

Yellowstone hawksbeard Modoc " $\%$

western hawksbeard

dandelion hawksbeard rattlebox

rusty rattlebox

yellow rattlebox
NOTE: Rattlebox is accepted common

crotalaria low crotalaria arrow crotalaria name for Daubentonia.

CROT-2 croton see also under TREES AND

Crco corymbulosus Engelm. croton

leatherweed croton

doveweed croton
Crte

texensis (Klotsch) Muell.

Texas croton

\section{CRYP \\ Cran \\ Crer \\ Crfu \\ Crja \\ Crmi}

Crra

\section{Cryptantha} angustifolia (Torr.) Greene

hiddenflower

crassisepala (Torr. \& Gray) Greene plains hiddenflower fulvocanescens (A. Gray) Payson beggarlice hiddenflower jamesii (Torr.) Payson micrantha (Torr.) Johnst. = Eremocarya James hiddenflower desertnut

James cryptantha micrantha (Torr.) Greene

NNOTE: Desertnut is SPN common name for the genus Eremocarya.

$$
\text { racemosa (s. Wats.) Greene }
$$

forgetmenot hiddenflower forgetmenot cryptantha

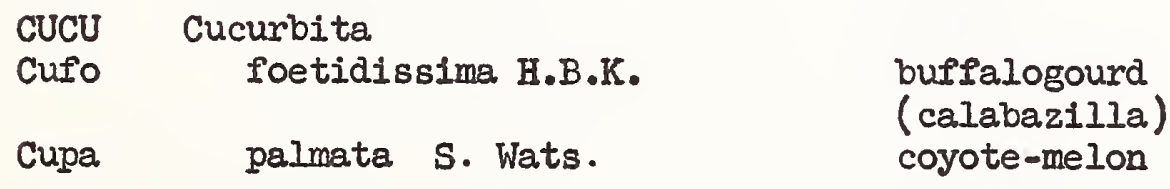



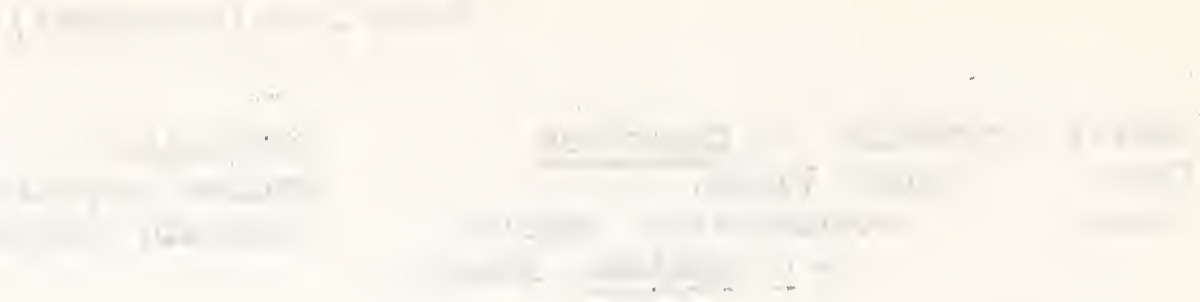

(n)

.
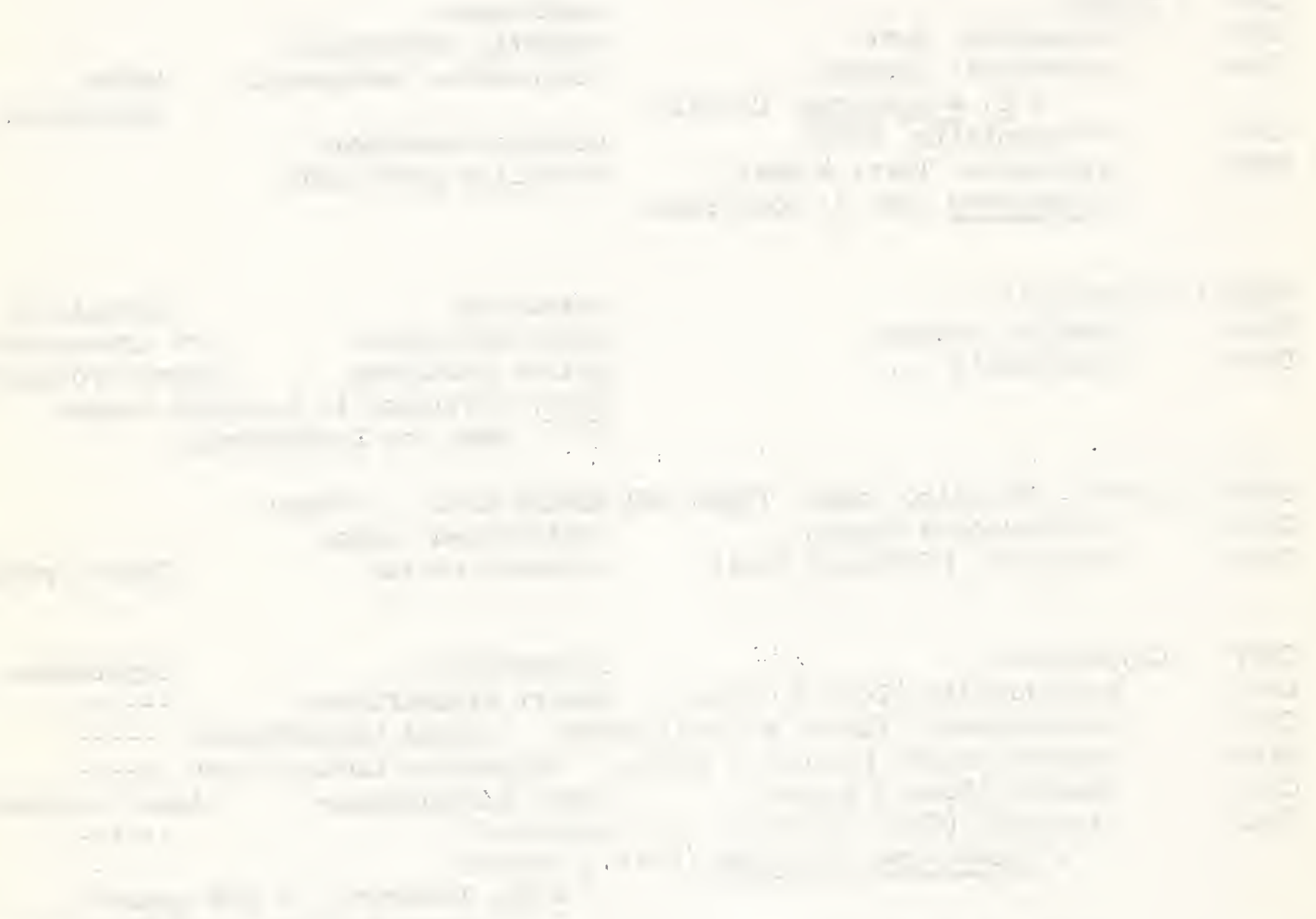

+
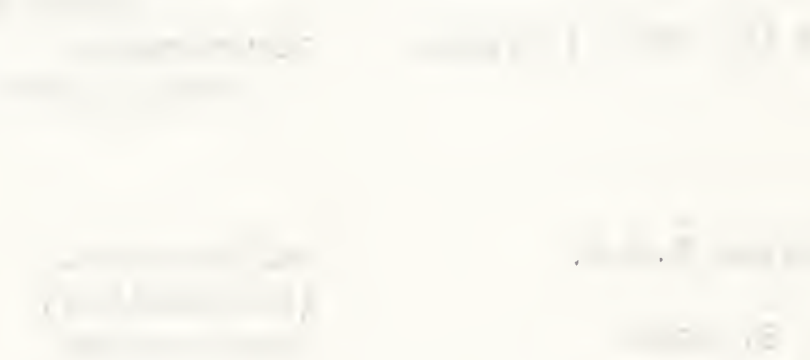
Forbs List - (continued)

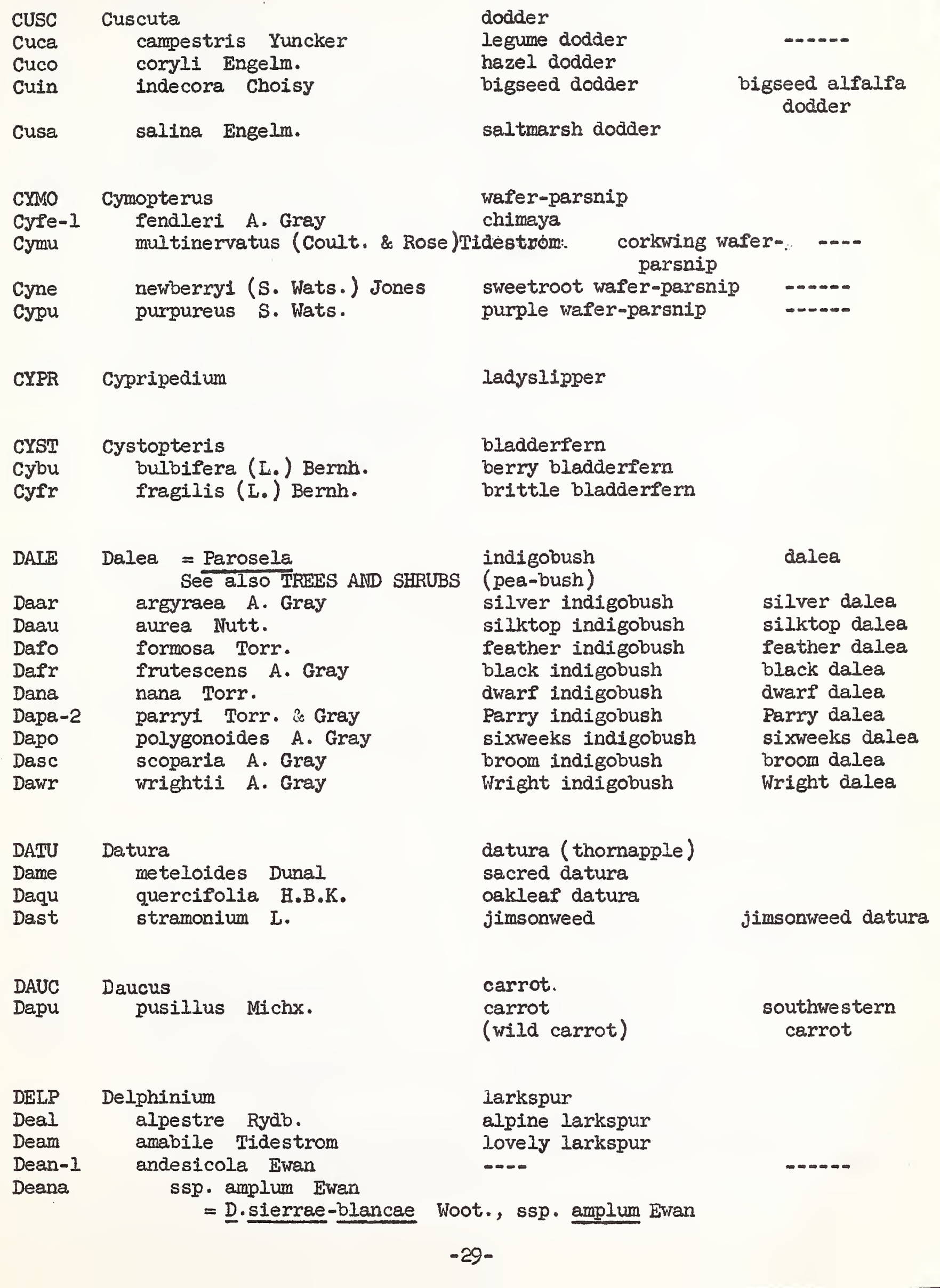




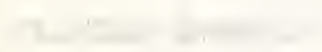
$-1+x$

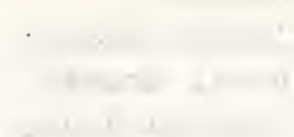

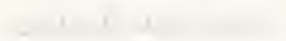

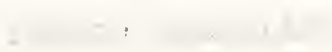

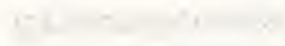

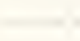

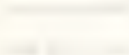
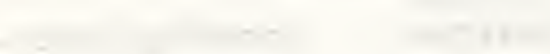

.

$+$

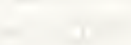

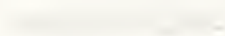

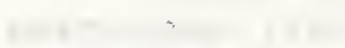

$\therefore$
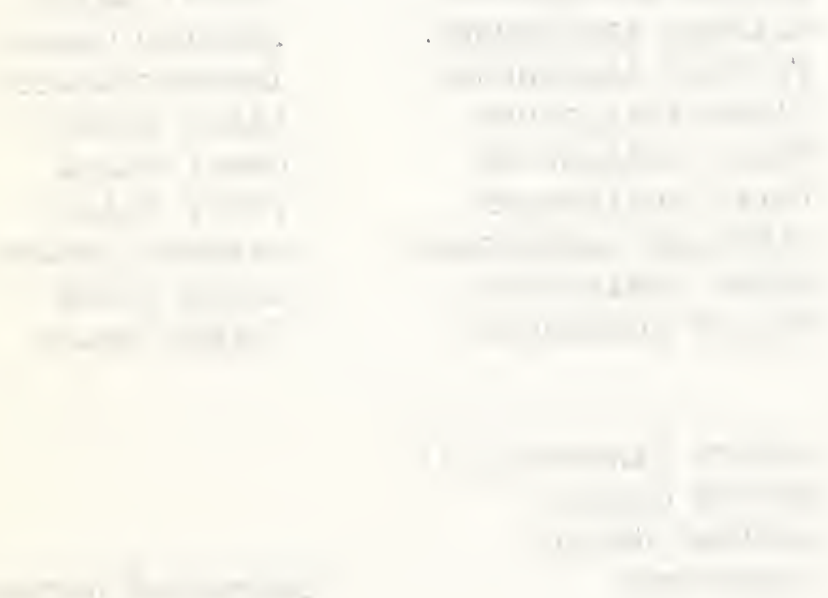

.

.
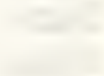

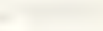

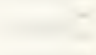

.

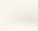

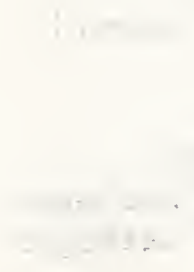




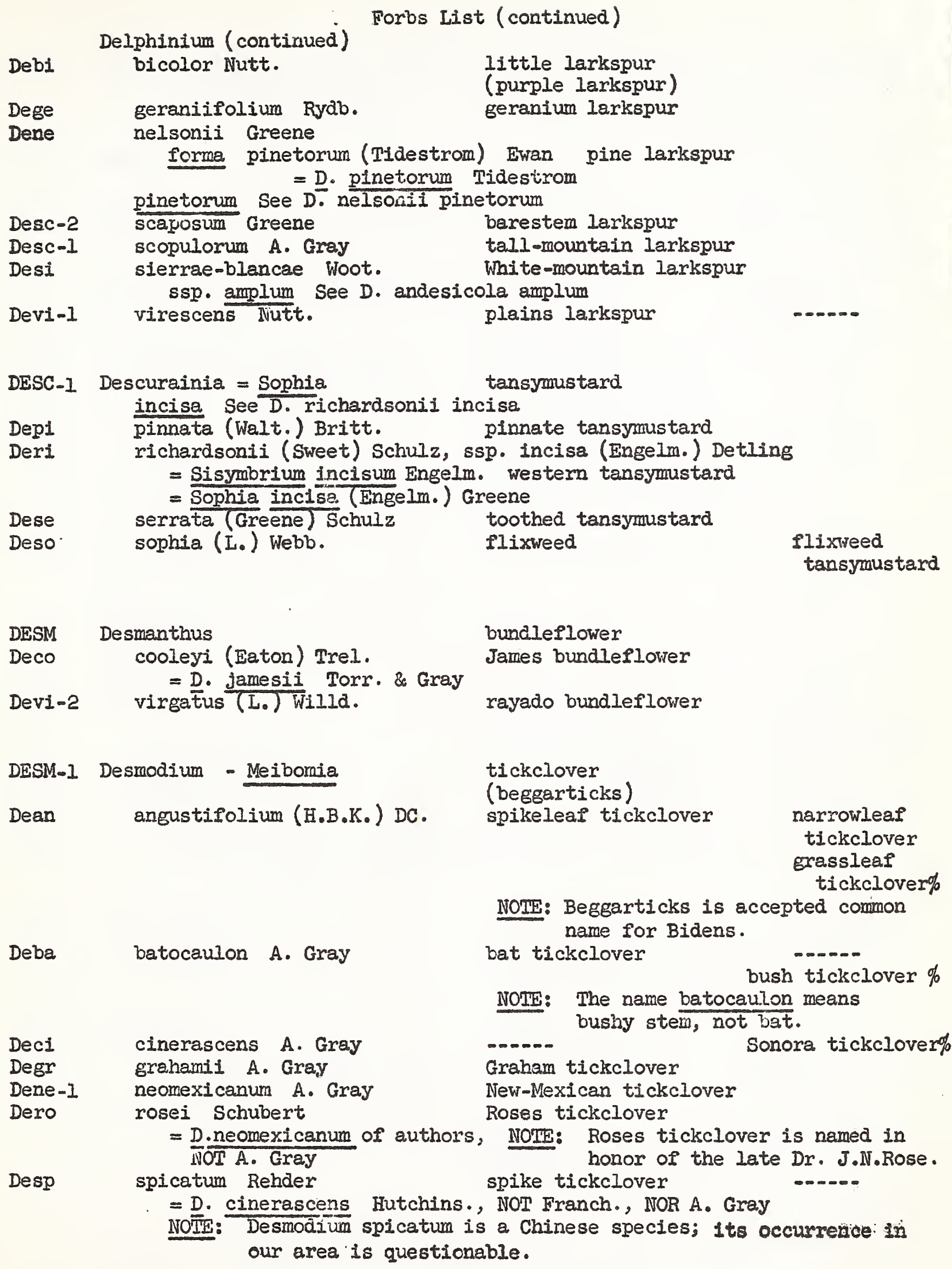




$$
\begin{aligned}
& \text { A16.. } \quad+\div \\
& \cdots+10+2+\infty
\end{aligned}
$$

\section{$-1$}

:

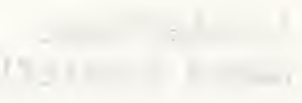
$+1,+1=0$ $+$

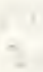
:

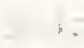

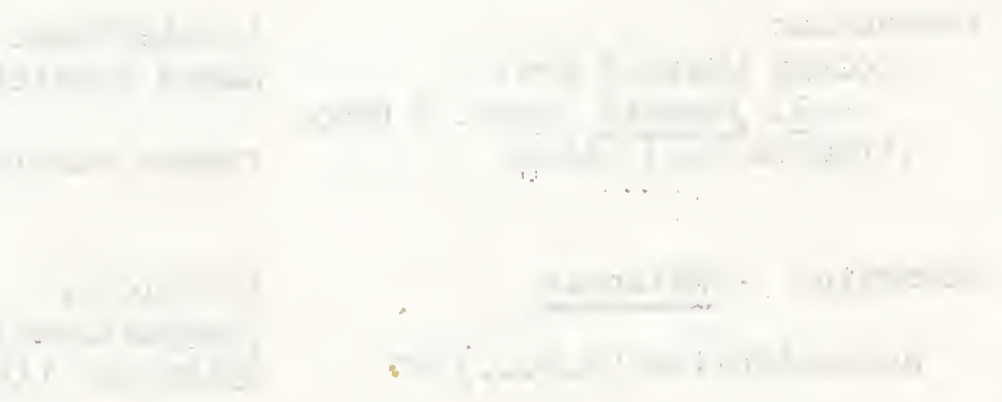

$$
\therefore+2+2+2
$$

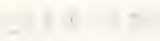

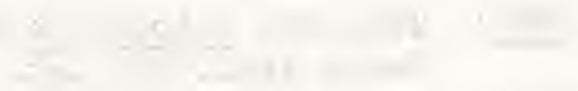

$$
\begin{aligned}
& \text { a }
\end{aligned}
$$

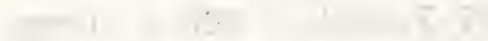
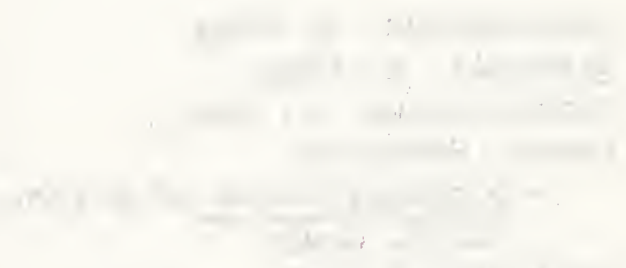

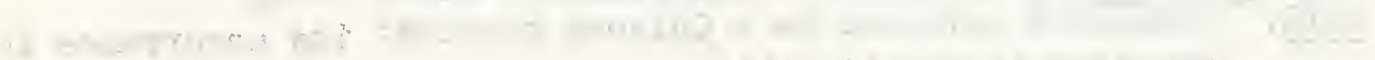

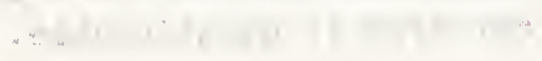




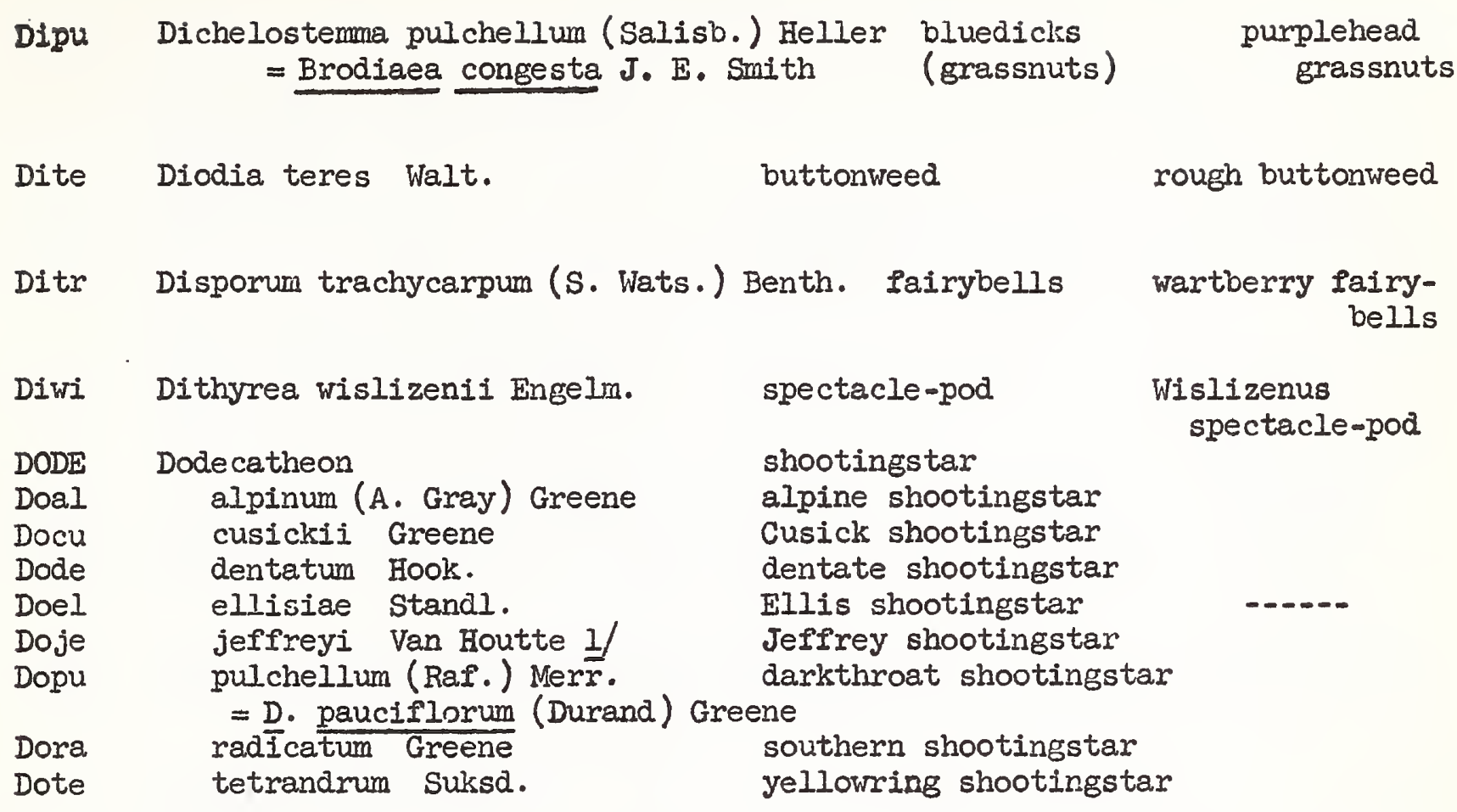

Dolicholus See Rhynchosia

Dondia See Suaeda.

DRAB Drabe

Drau

Drer

Drne

Drst

Drstn aurea M. Vahl crassifolia R. Grah. nemorosa $L$. stenoloba Ledeb.

var. nana (Schulz) C.I.Hitche. shiny whitlowgrass = D. nitida Greene whitlowgrass

(whitlowwort)

golden whitlowgrass

hairy whitlowgrass

woods whitlowgrass draba.

(whitlowwort) golden draba

-...-.

woods draba

shiny draba
DRAC DracocephaIum

DRYM Drymaria

Drfe fendleri $S$. Wats. Drho holosteoides Benth.

Drpa pachyphylla Woot. \& Standl. false-dragonhead NNOTE: False-dragonhead is common name for Moldavica.

arymary

Fendler drymary

thickleaf arymary

poison drymary

Mexican drymary *

thickleaf

aryinary*

*NOTE: These two species may be

-synonymous, as standardized Plant

Names ( $p .202$ ) indicates. yet located.

I/ Moore given as author in some manuals; original description not 


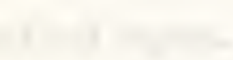

$\sqrt{1}+$

$\sqrt{2}$

+

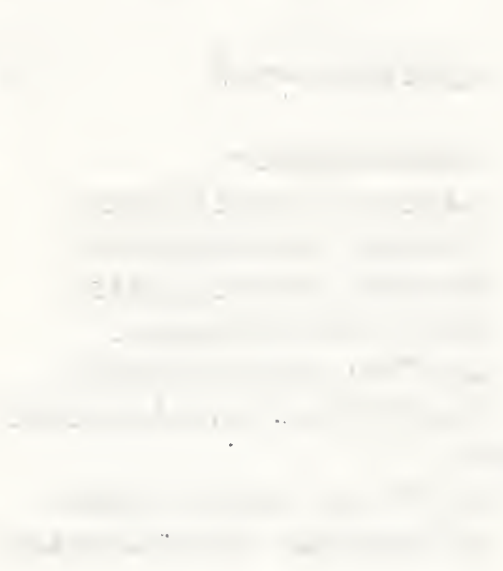

nh
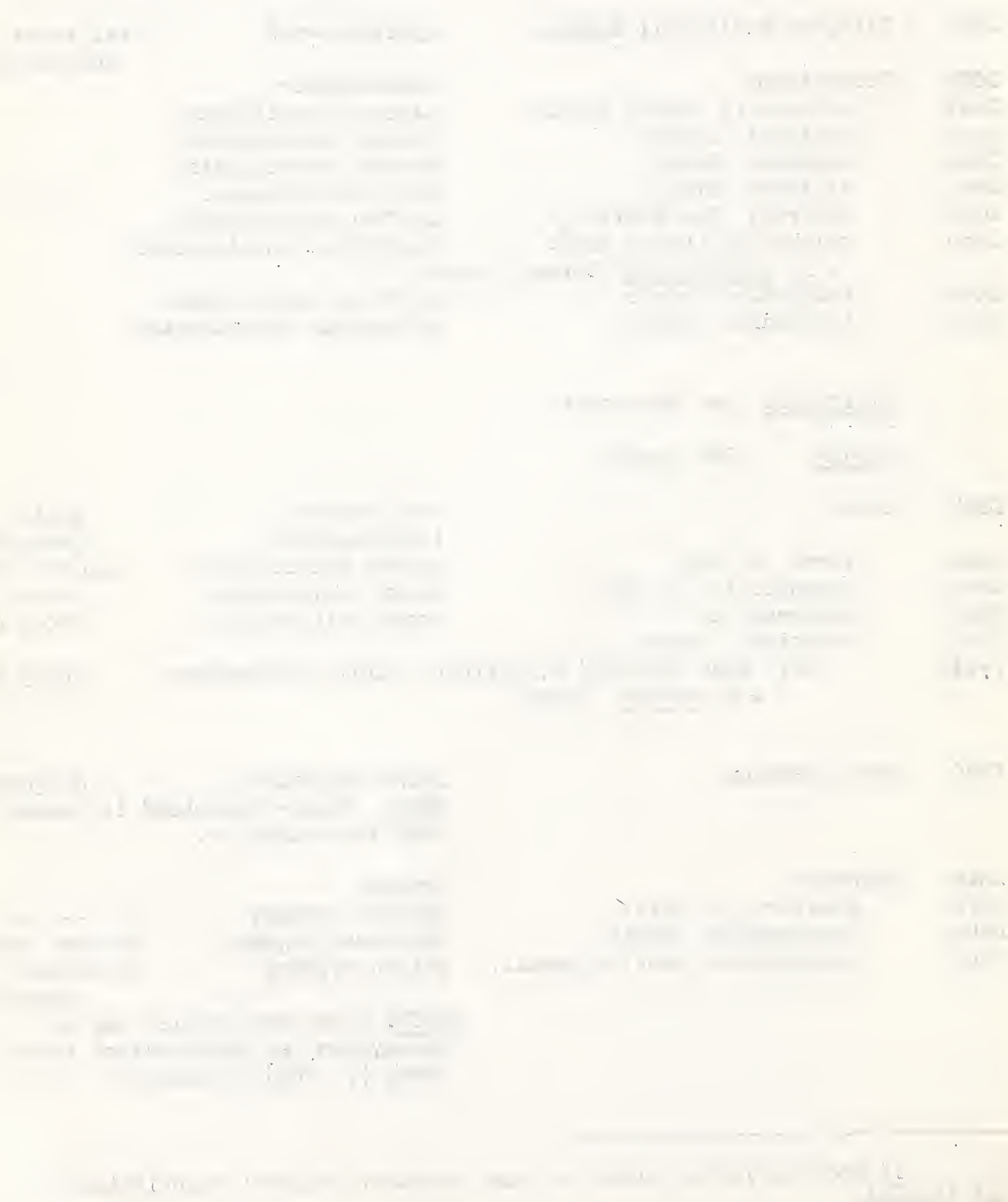

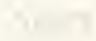

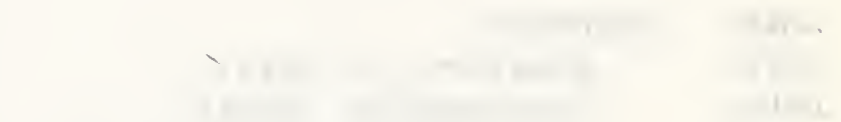




\section{Forbs List (continued)}

Drymocallis See Potentilla

DRYO Dryopteris

shieldfern

woodfern

Echinocactus See "Shrubs"

Echinocereus See "Shrubs"

Eclo Echinocystis lobata (Michx.) Torr. \& Gray mock-cucumber Wild mock-

Ecwr Echinopepon wrightii (A. Gray)s. Wats. spinycucumber cucumber

Echy Echinopsilon hyssopifolius (Pall.) Moq. smotherweed fivehook bassia Bassia hyssopifolia (Pall.) Kintze

Ephedra See "shrubs"

EPII

Epad

Epal

Epan

Epbr

Epho

Eppa

Epgi Epipactis gigantea Dougl. willowrweed

sticky willowweed

alpine willowweed

fireweed

("blooming-sally")

Sierra willowweed

Hornemann willotiveed

autumn wi工lowweed

helleborine strean epipactis NOTE: Giant helleborine is suggested if helleborine preferred to epipactis.

horsetail

fiela horsetail

scouring-rush

stout scouring-rush

smooth horsetail

Eqla

arvense $I_{\text {. }}$

hyemale $\mathrm{L}$. elatum (Engelm.) Fiorton

= E. praealtum Raf.

laevigatum A. Braun

praealtum See $E_{\text {. hyemale elatum }}$

Eremocarya micrantha See Cryptantha micrantha 


\section{$-1+2+-$}

1

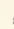

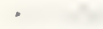

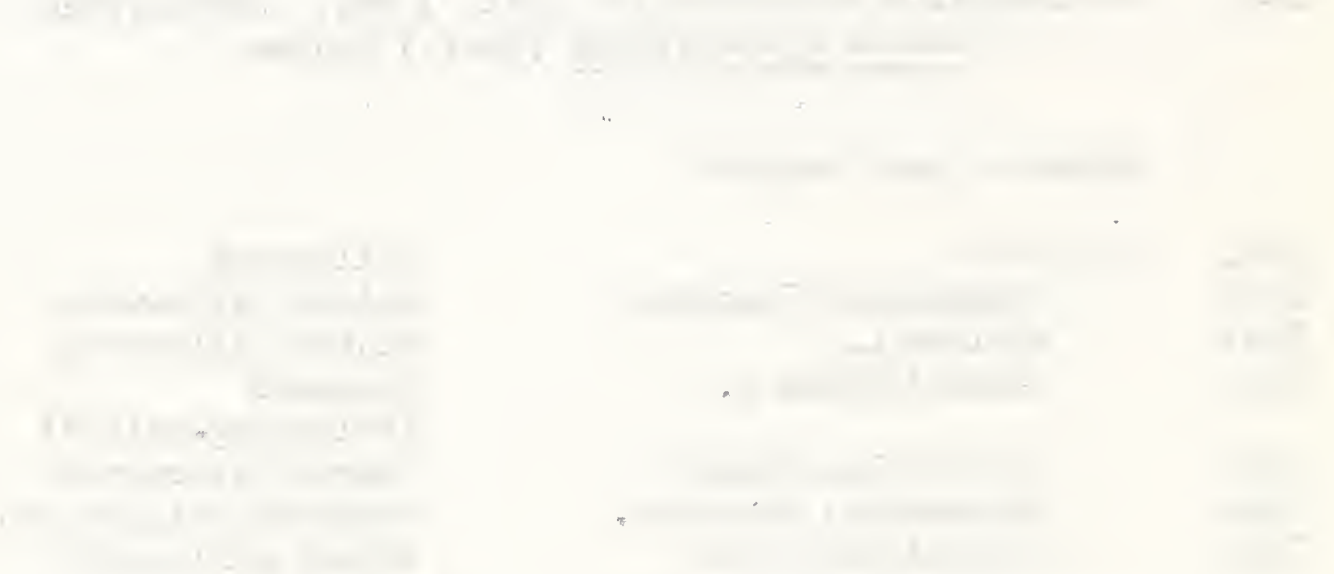

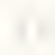
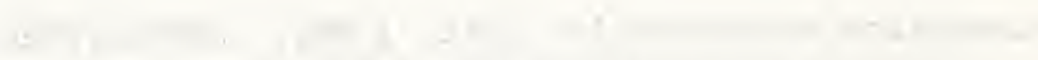

1
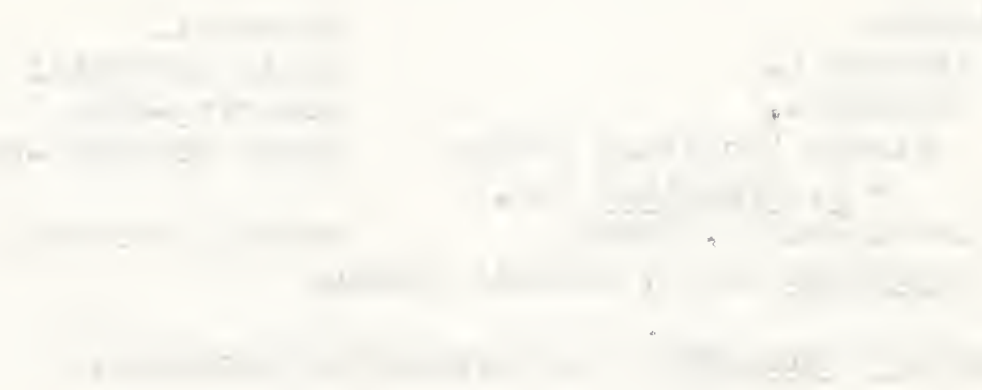
Forbs List (continued)

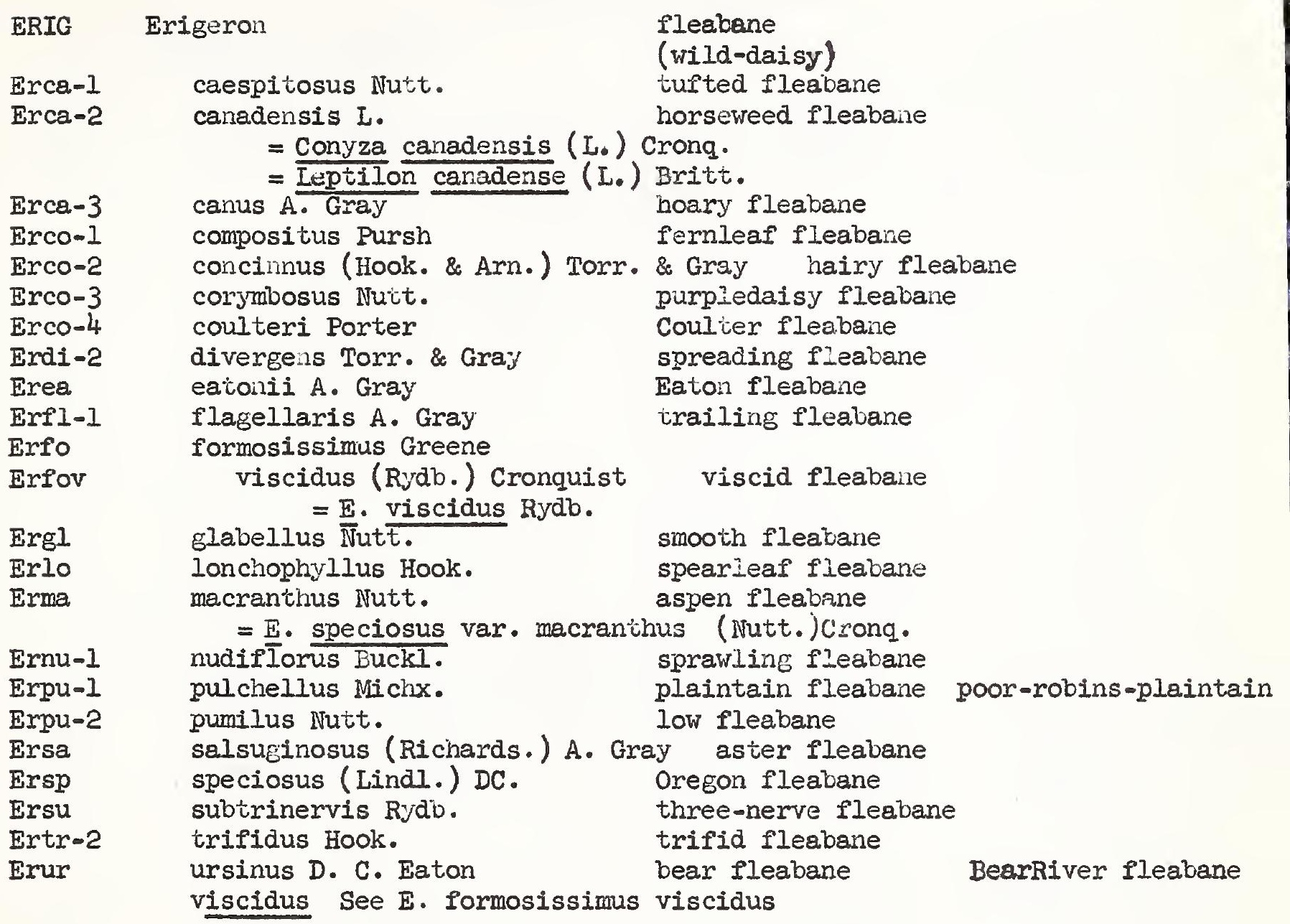

ERIO-1 Eriogonum

buckwheat

(sulfur flowers)

eriogonum

NOTE: Buckwheat is the officially accepted common name for Fagopyron; if the use of buckwheat is especially desired in the common name for Eriogonum, wildbuckwheat is suggested to indicate the plant is not a true buckwheat.

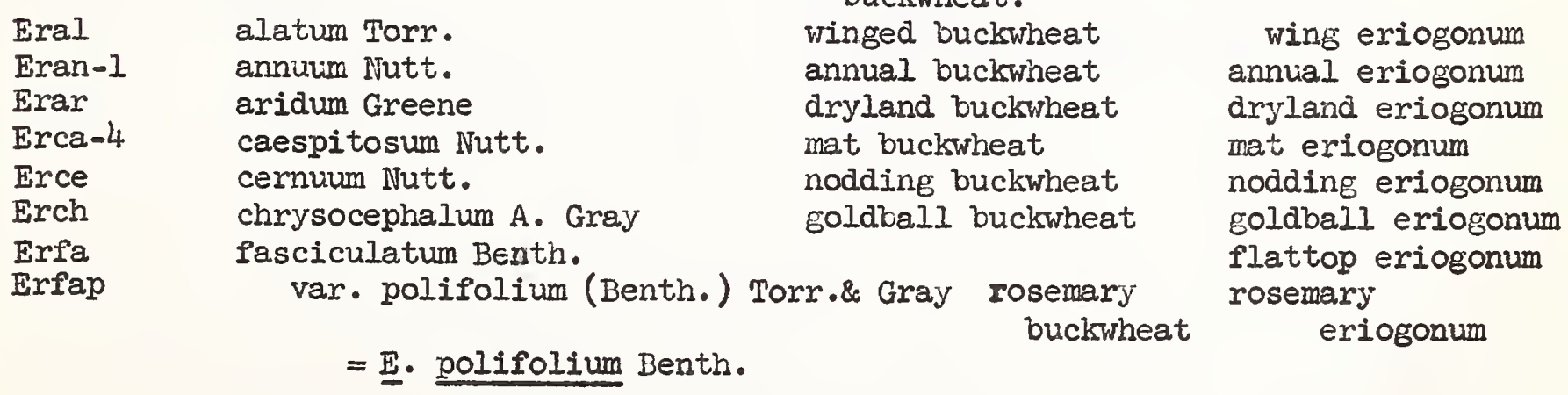



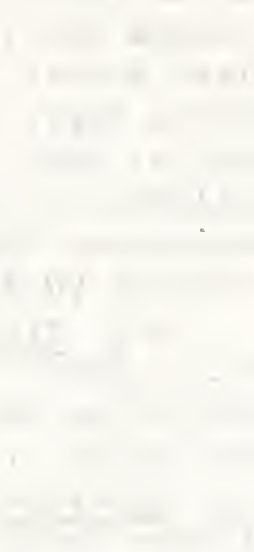

$-1$

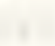

$+2$

1

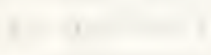

, n n n 
Forbs List (continued)

$\begin{array}{llll} & \text { Eriogonum (continued) } & \text { buckwheat } & \text { eriogonum } \\ \text { Erfl-2 } & \text { flavum Nutt. } & \text { yellow buckwheat } & \text { yellow eriogonum } \\ \text { Erhi } & \text { hieracifolium Benth. } & \text { Wyeth buckwheat } & \text { - W }\end{array}$

\begin{tabular}{|c|c|c|c|}
\hline Erin-2 & inflatum Torr. \& Frem. & deserttrumpet buckwheat & deserttrumpet \\
\hline Erja & jamesii Benth. & antelope-sage & at James \\
\hline Ermi & microthecum Nutt. & slender/buckwheat & $\begin{array}{l}\text { slenderbush } \\
\text { eriogonum }\end{array}$ \\
\hline Ernu-2 & nudum Benth. & barestem buckwheat & $\begin{array}{l}\text { barestem } \\
\text { eriogonum }\end{array}$ \\
\hline Erov & ovalifolium Mutt. & cushion buckwheat & $\begin{array}{l}\text { cushion } \\
\text { eriogonum }\end{array}$ \\
\hline Erph & pharnaceoides Torr. & wirestem buckwheat & $\begin{array}{l}\text { wirestem } \\
\text { eriogonum }\end{array}$ \\
\hline Erpi -1 & $\begin{array}{l}\text { piperi Greene } \\
\text { polifolium See } E_{\text {. fasciculat }}\end{array}$ & $\begin{array}{l}\text { Piper buckwheat } \\
\text { um polifolium }\end{array}$ & Piper eriogonum \\
\hline Erpo & polycladon Benth. & sorrel buckwheat & sorrel eriogonum \\
\hline Erra & racemosum Nutt. & redroot buckwheat & redroot eriogonum \\
\hline Erst & stellatum Benth. & Iongray buckwheat & Iongray eriogonum \\
\hline Ersu $=1$ & subalpinum Greene & subalpine buckwheat & $\begin{array}{l}\text { subalpine } \\
\text { eriogonum }\end{array}$ \\
\hline Ertr -3 & tripodum Greene & Lake buckwheat & $\begin{array}{l}\text { Lake eriogonum } \\
\text { tripod eriogonum) }\end{array}$ \\
\hline Erum & umbellatum Torr. & sulfur bucksheat & sulfur eriogonum \\
\hline Ervi & vimineum Dougl. & broom buckwheat & broom eriogonum \\
\hline Erwr & wrightii Torr. & Wricht buckwheat & Wright eriogonum \\
\hline $\begin{array}{l}\text { ERIO-2 } \\
\text { Erco-5 }\end{array}$ & $\begin{array}{l}\text { Eriophyllum } \\
\text { confertiflorum (DC.) A. Gray }\end{array}$ & $\begin{array}{l}\text { woollydaisy } \\
\text { goldenyarrow } \\
\text { woollydaisy }\end{array}$ & $\begin{array}{l}\text { eriophyllum } \\
\text { goldenyarrow } \\
\text { eriophyllum }\end{array}$ \\
\hline $\begin{array}{l}\text { EROD } \\
\text { Erci-1 }\end{array}$ & $\begin{array}{l}\text { Erodium } \\
\quad \text { cicutarium (L.) L'Her. }\end{array}$ & \multirow{2}{*}{\multicolumn{2}{|c|}{$\begin{array}{l}\text { heronbill } \\
\text { filaree (alfileria) alfileria } \\
\text { (heronsbill) (Pinclover) } \\
\text { Texas heronbill }\end{array}$}} \\
\hline Erte & texanum A. Gray & & \\
\hline
\end{tabular}


4. + +

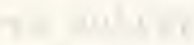

,

$+$

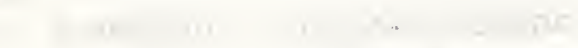

$\sqrt{10}$

(10

$-$

$\because+1+1+1+1$

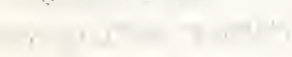

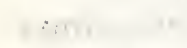

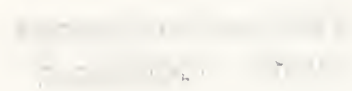

$+4+1+2$

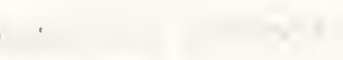

(1)
1

$-$

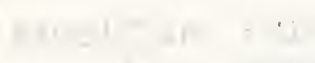

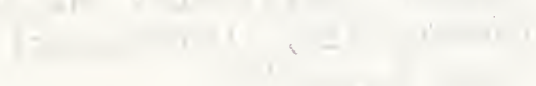

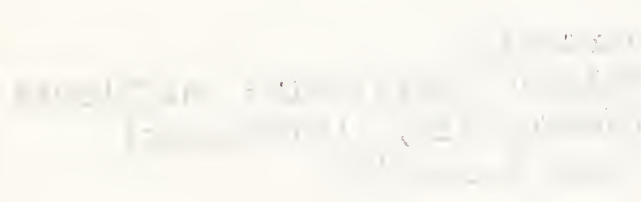

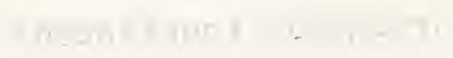

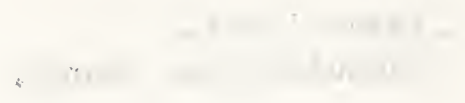

.

$1+$

$\sqrt{1}+1$

$+10$
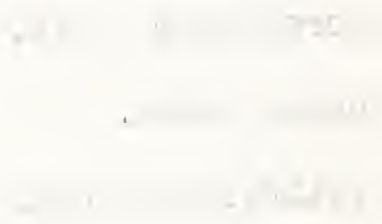

i 1
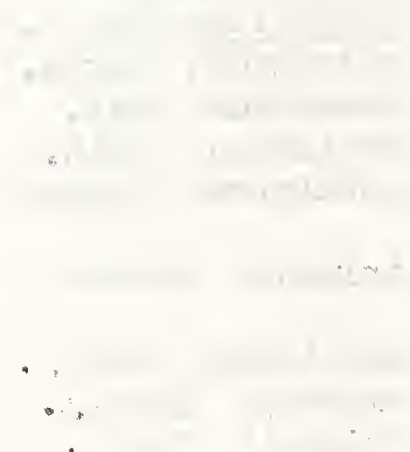

,

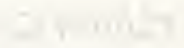

$1-1$

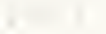

(n)

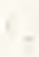

15 the
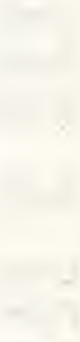

r
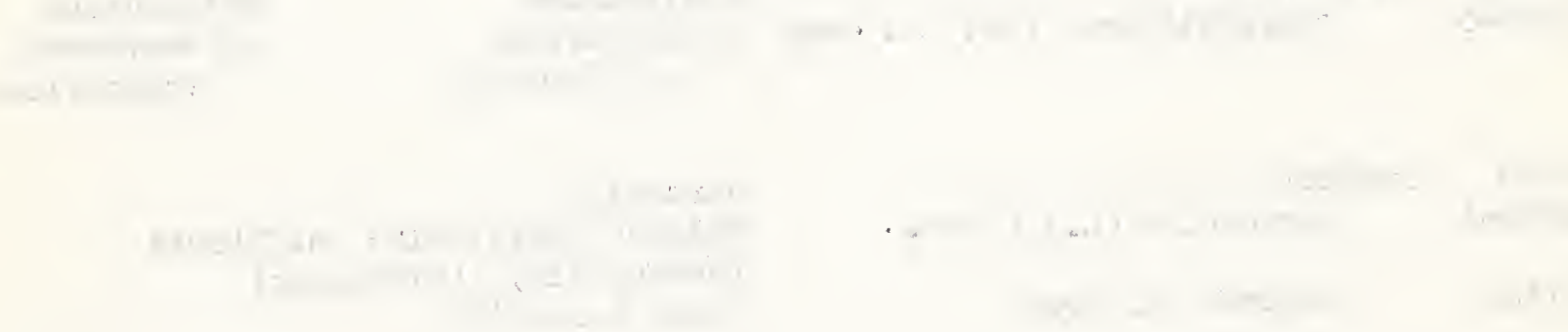
Forbs List (continued)

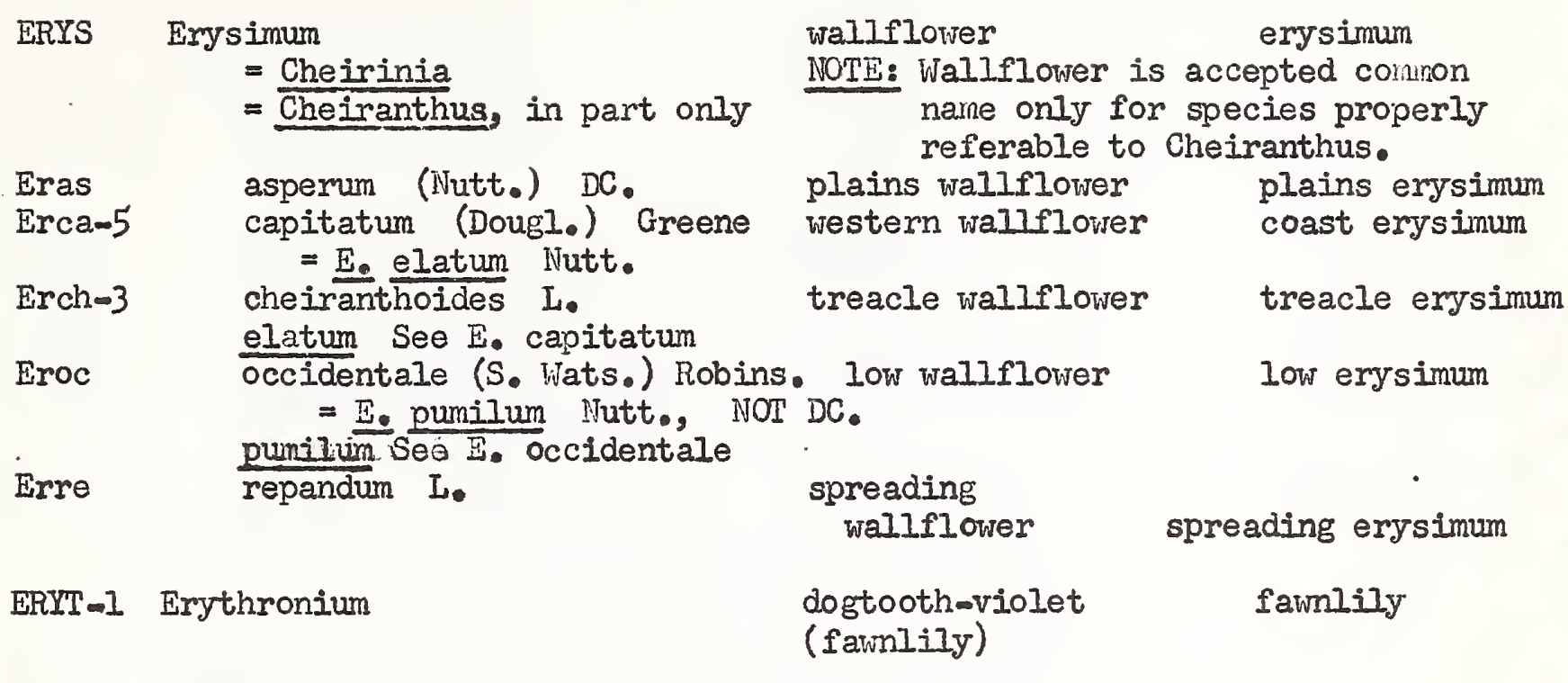

Eschenbachia See Conyza

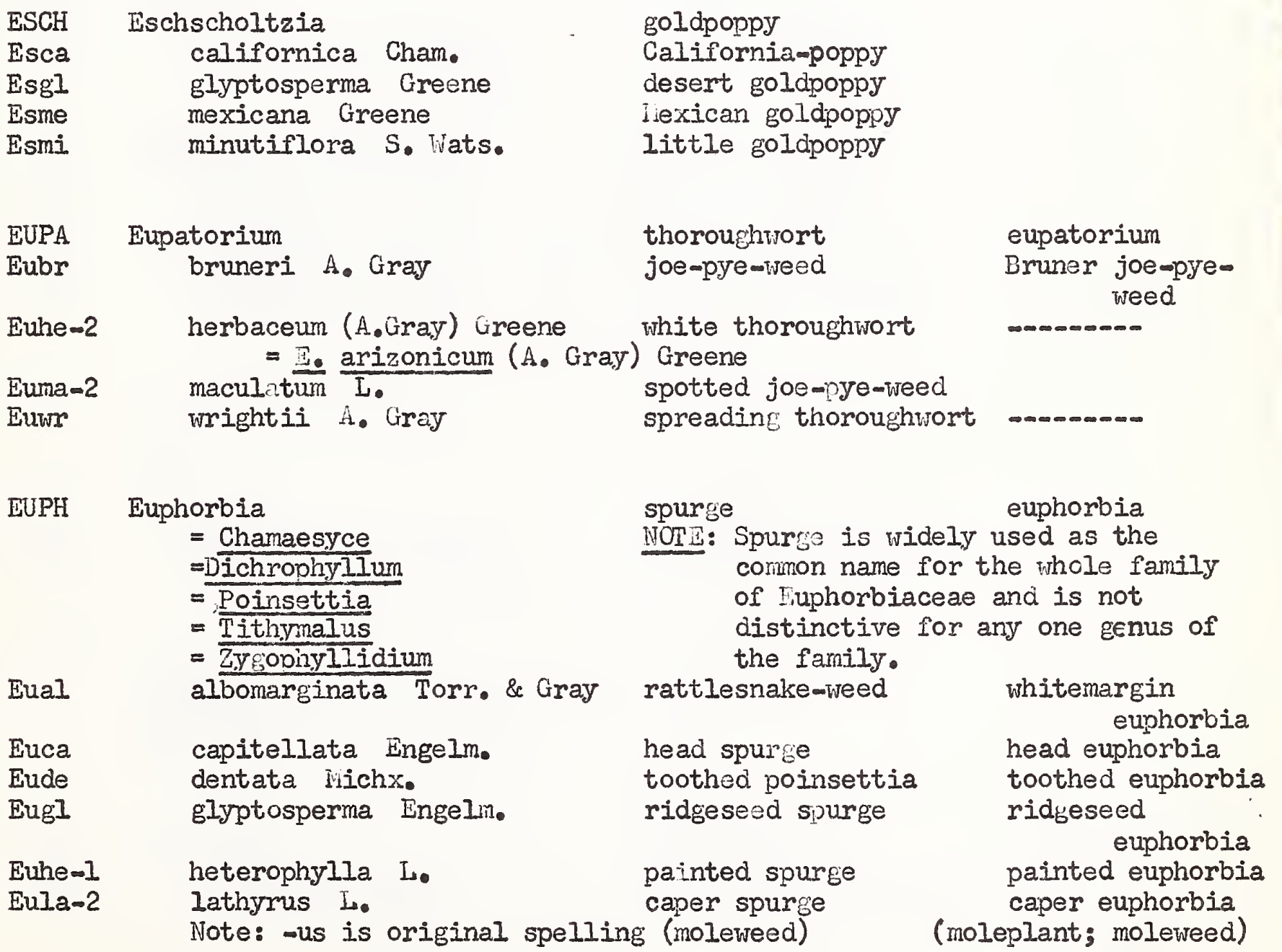




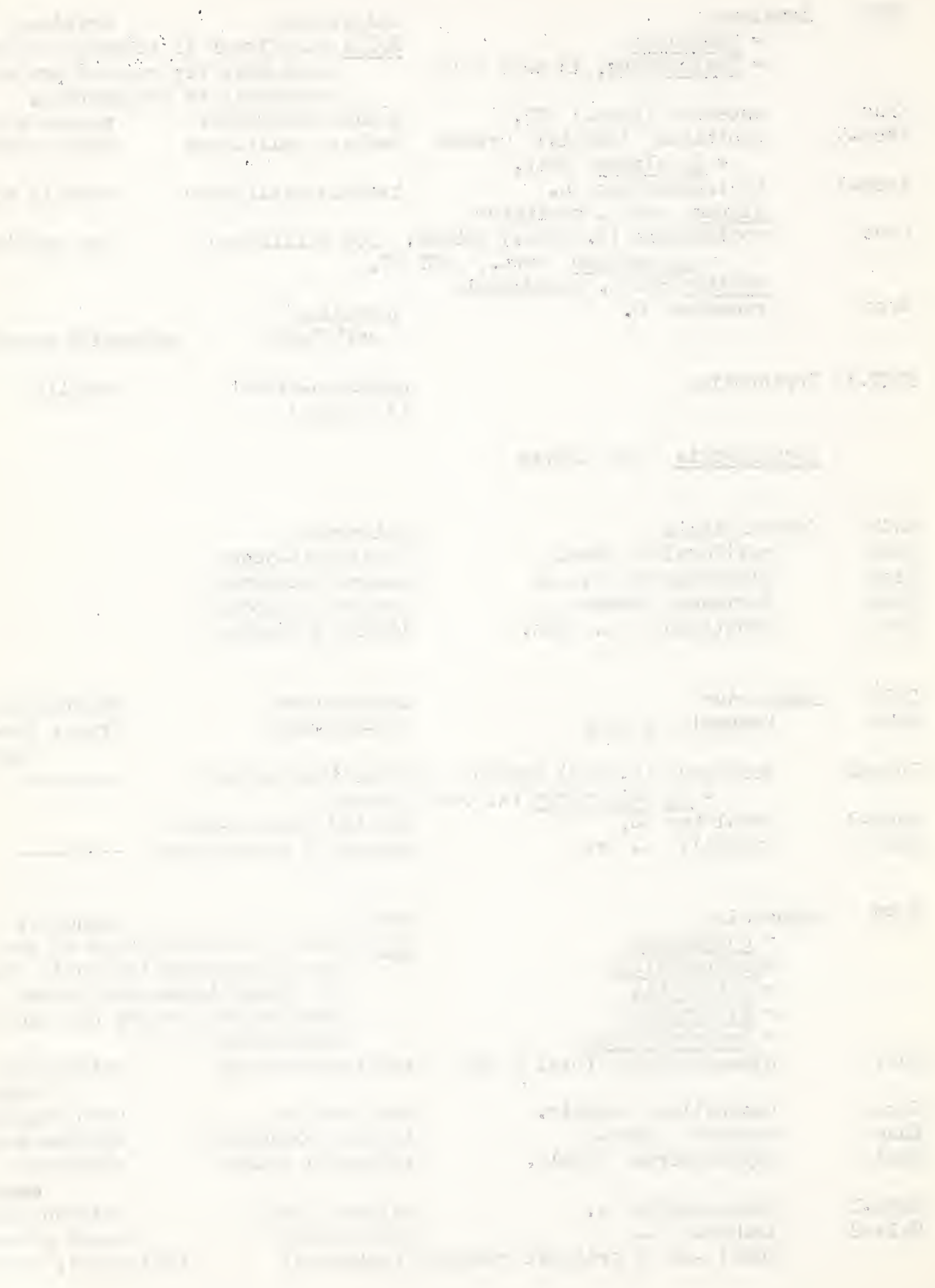


Forts List (Continued)

\begin{tabular}{|c|c|c|}
\hline \multicolumn{2}{|c|}{ Euphorbia (continued } & spurge \\
\hline Euma-1 & marginata Pursh & snow-on-the-mountain \\
\hline $\begin{array}{l}\text { Eupe } \\
\text { Eupu }\end{array}$ & $\begin{array}{l}\text { peplus Is. } \\
\text { pulcherrima Willd. }\end{array}$ & $\begin{array}{l}\text { petty spurge } \\
\text { poincettia }\end{array}$ \\
\hline $\begin{array}{l}\text { Euro } \\
\text { Euse-1 } \\
\text { Euse-2 }\end{array}$ & $\begin{array}{l}\text { robusta (Engelin.) Small } \\
\text { serpens H.B.K. } \\
\text { serpyllifolia Pers. }\end{array}$ & $\begin{array}{l}\text { robust spurge } \\
\text { serpent spurge } \\
\text { thymeleaf spurge }\end{array}$ \\
\hline use -3 & setiloba Engelm. & Yuma spurge \\
\hline
\end{tabular}

euphorbia

snowmon-them mountain euphorbia petty euphorbia common poinsettia robust euphorbia serpent euphorbia thymeleaf euphorbia Yuma euphorbia

Euploca convolvulacea See Heliotropium convolvulaceum

FLOE Floerkea

FRAG Fragaria

Frbr

Frov

FRAN

Frdu

Frer

Fril

Frte

Frto

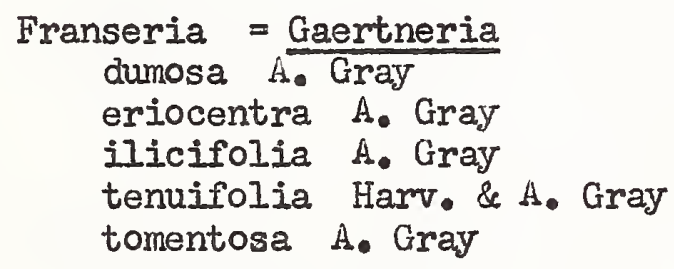

false-mermaid

strawberry

bracted strawberry

wild strawberry

bur-sage

white bur-sage

woolly bur-sage

hollyleaf bur-sage

slimleaf bur-sage

woolleaf bur-sage woollyleaf bur-sage

Frasera See Swertia

FRIT

Frat

Frpu

FROE Froelichia

Funastrum See Philibertia

Gaertneria See Franseria fritillary

purplespot fritillary

yellow fritillary

snakecotton $\begin{array}{lc}\text { blanketflower } & \text { gaillarida } \\ \text { blanketflower } & -\ldots-\ldots \\ \text { firewheel (rose-ring) } & \text { rose-ring } \\ & \text { gaillardia }\end{array}$
GAIL

Gapi

Gapu
Gaillardia

pinnatificia Torr.

pulchella Foug. 
. Forto: Iist (continued)

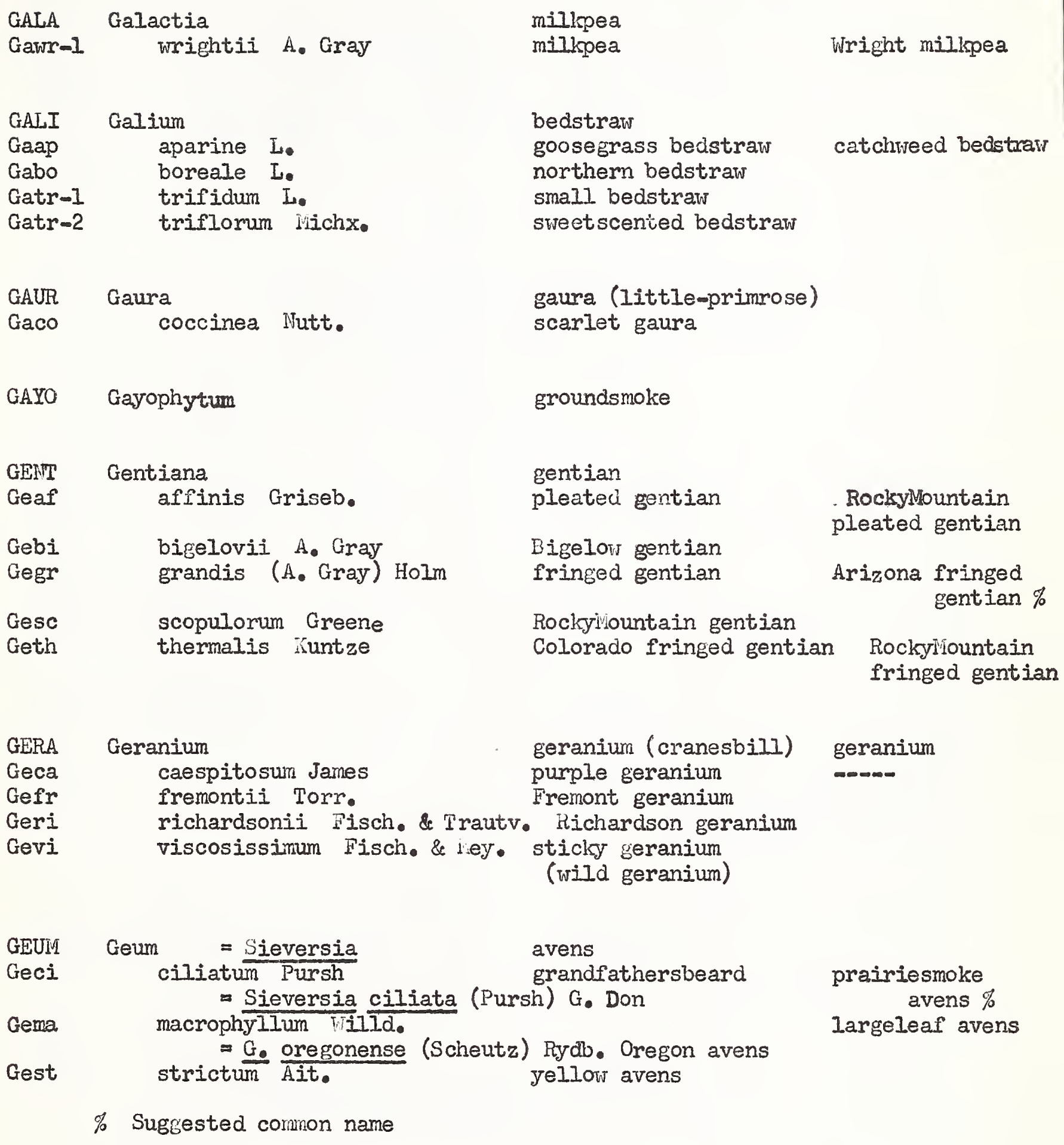




\section{-}

$+12$

$-+$ 


$$
\text { Forbs: List (continued) }
$$

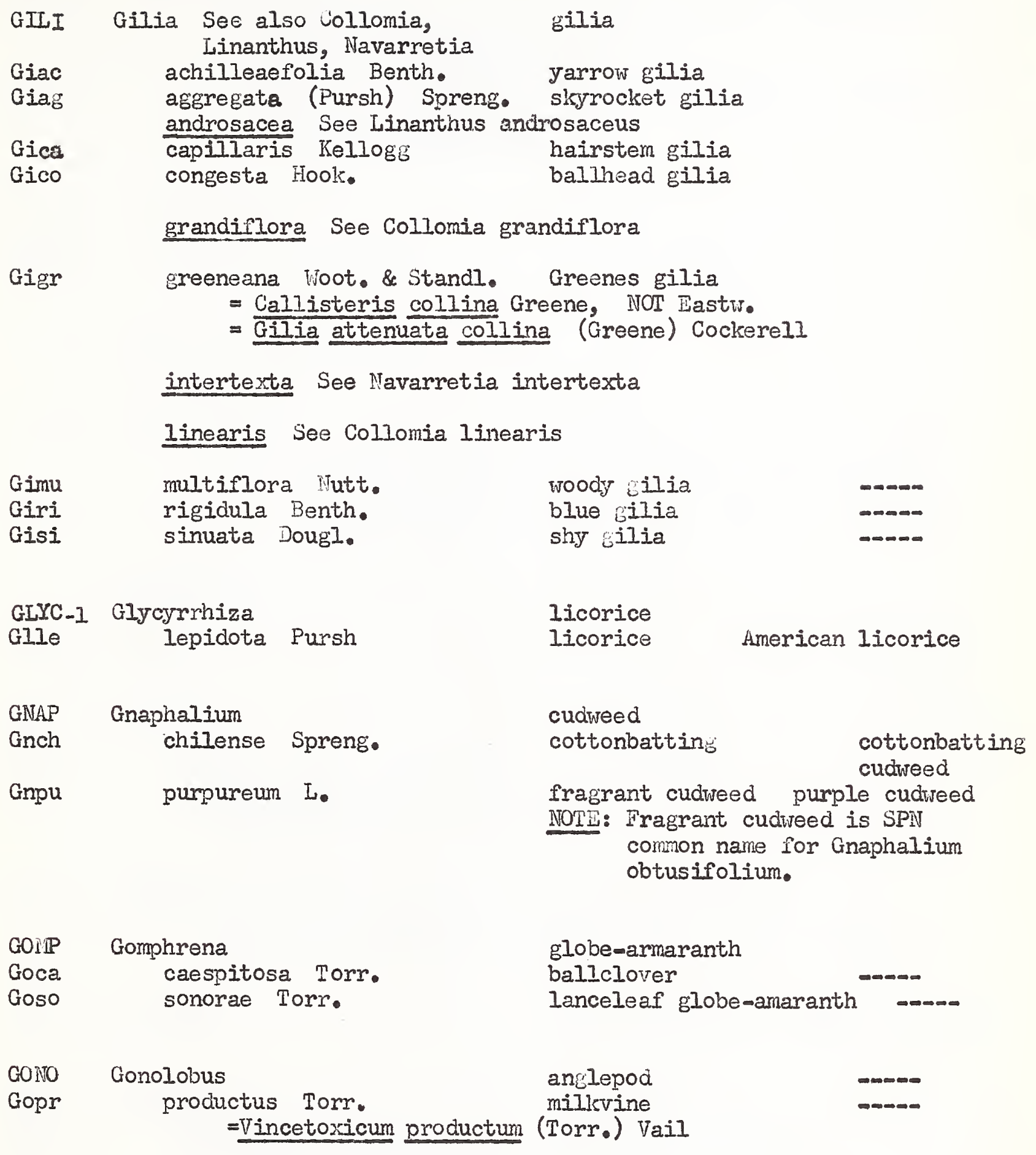




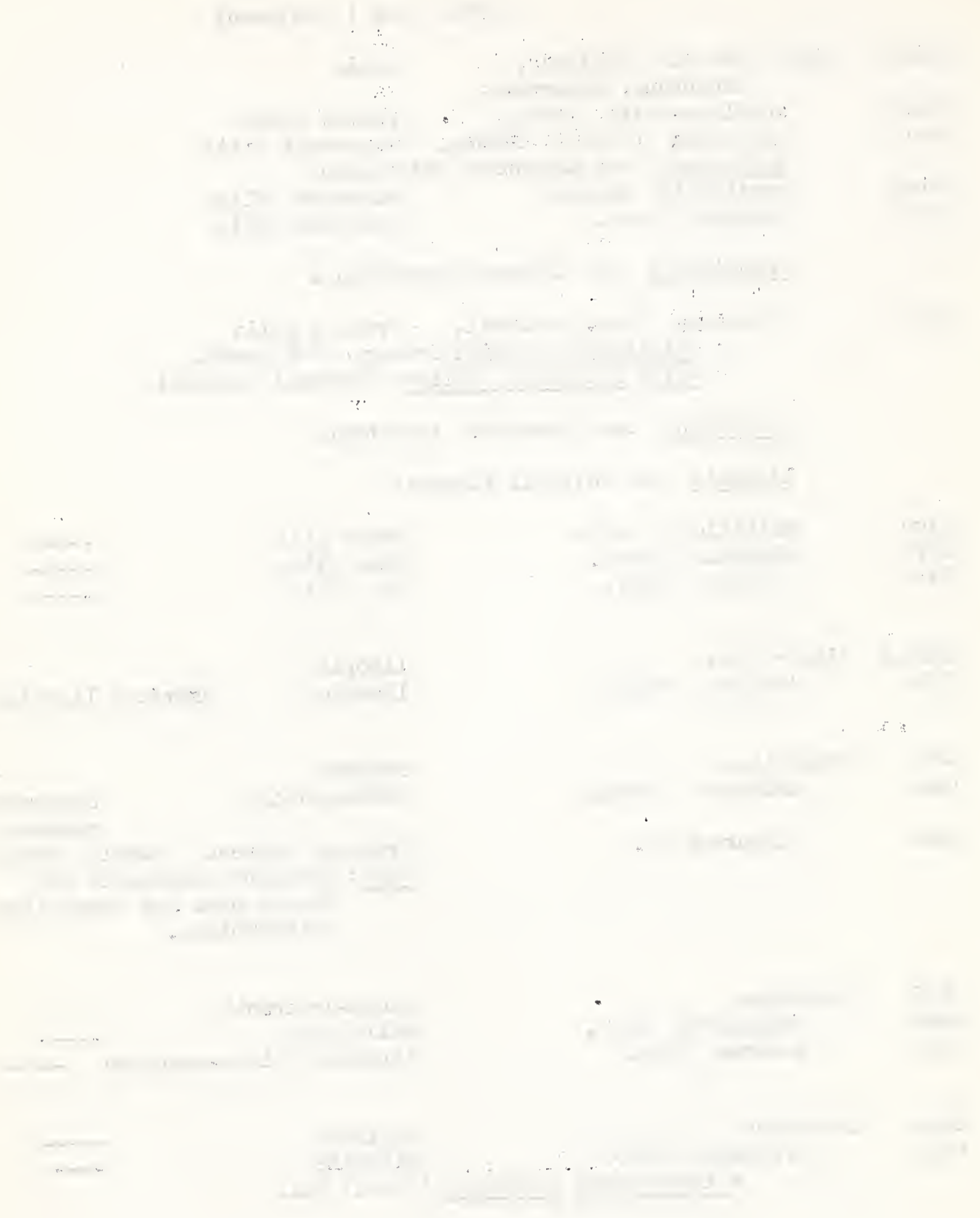




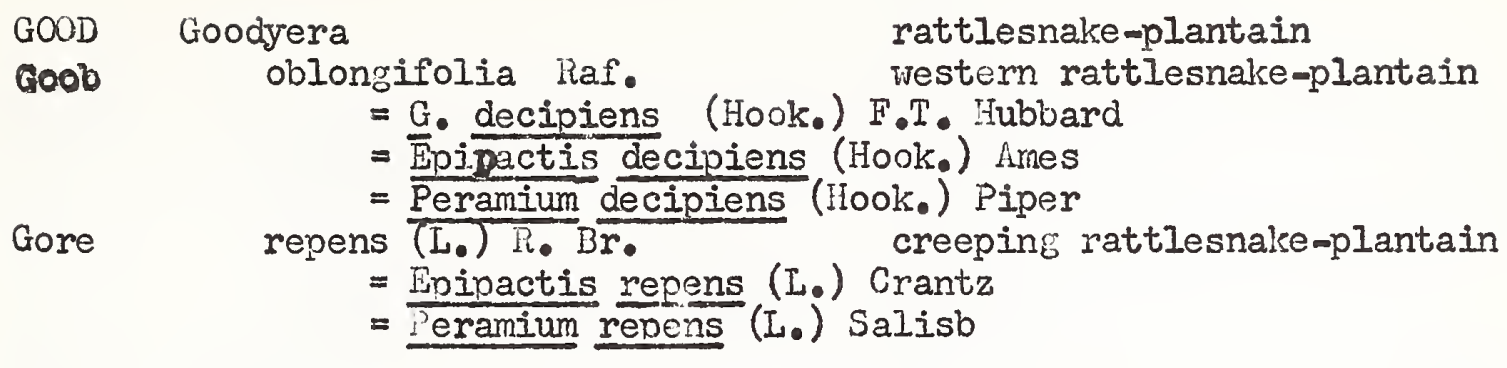

\begin{tabular}{lrll} 
GRIN & Grindelia & \multicolumn{3}{c}{ gumireed } \\
Grar & arizonica & A. Gray & Arizona gumweed \\
Grsq & squarrosa & (Pursh) Dunal curlycup gumweed
\end{tabular}

Gutierrezia See "Shrubs"

GYNW

$$
\begin{aligned}
& \text { Gymnocarpium oakiern } \\
& \text { NOIE: Oakfern is the SPN ccrmon name for Dryopteris }
\end{aligned}
$$

Gymnolomia See Viguiera

HABE Habenaria

HALE Halenia

Hare bog-orchid

habenaria

spuragentian

spuragentian

Hacr Haplophyton crooksii L. Benson cockroach-plant $=\mathrm{H}$. cimicidum of some authors, NOT A. DC. which is a Wexican and Guatemalan species.

HEDE Hedeoma

HELE Helenium

Heho hoopesii A. Gray

Hete tenuifolium Nutt.

HELI-2 Helianthella mock-pennyroyal false-pennyroyal

sneezeweed

orange sneezeweed

bitter sneezeweed 

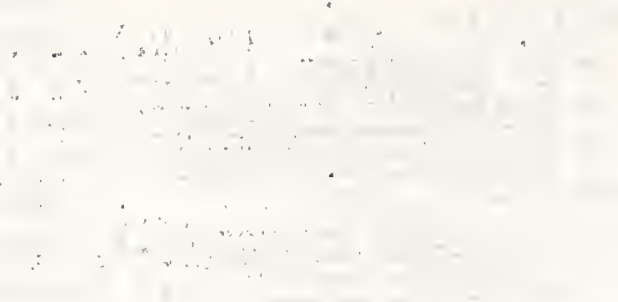

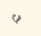

$m:$

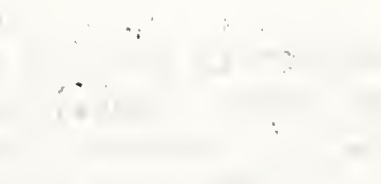

r
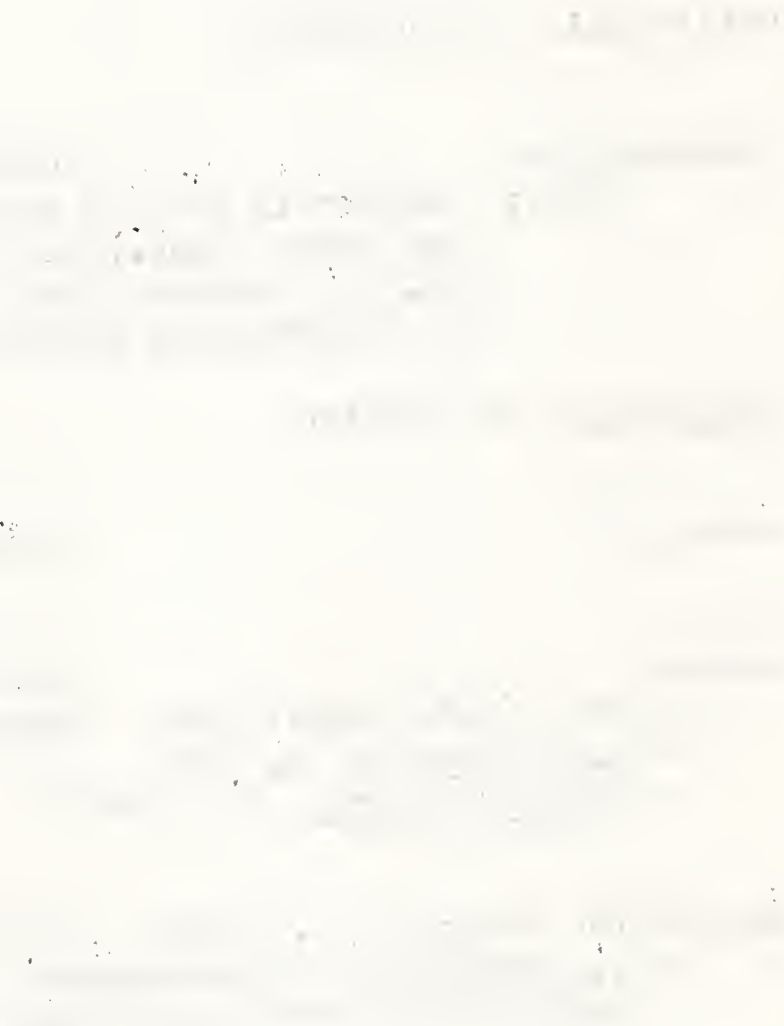


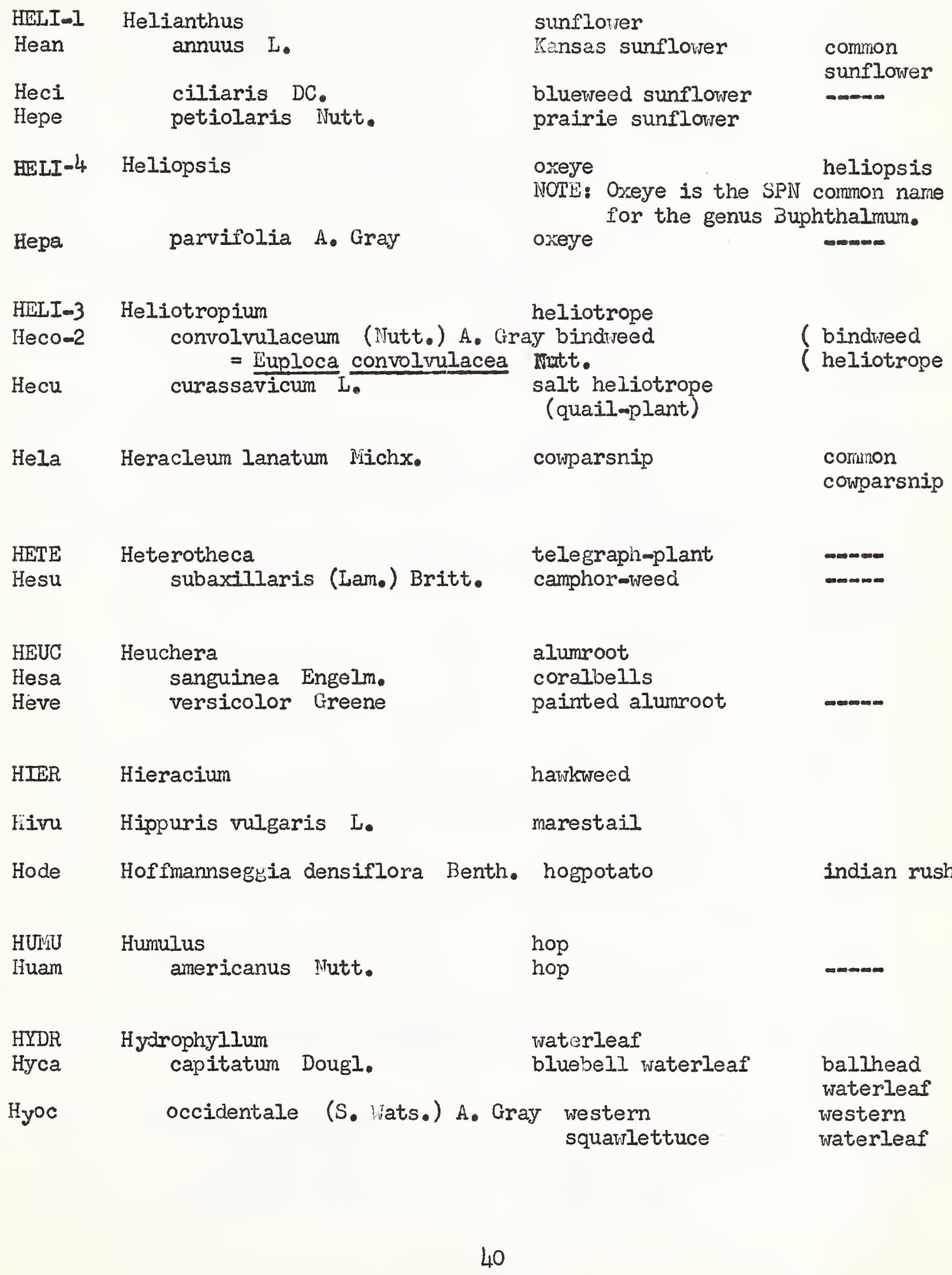




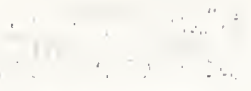

$+\quad+\cdots$,

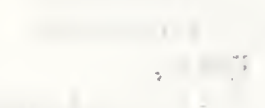

$+1-1$

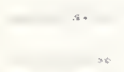

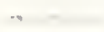

$\cdots$

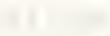

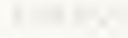

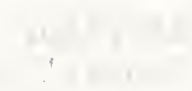

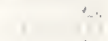

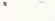

+

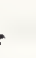

,

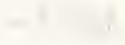

$\therefore+1=$ 
Forbs List (continued)

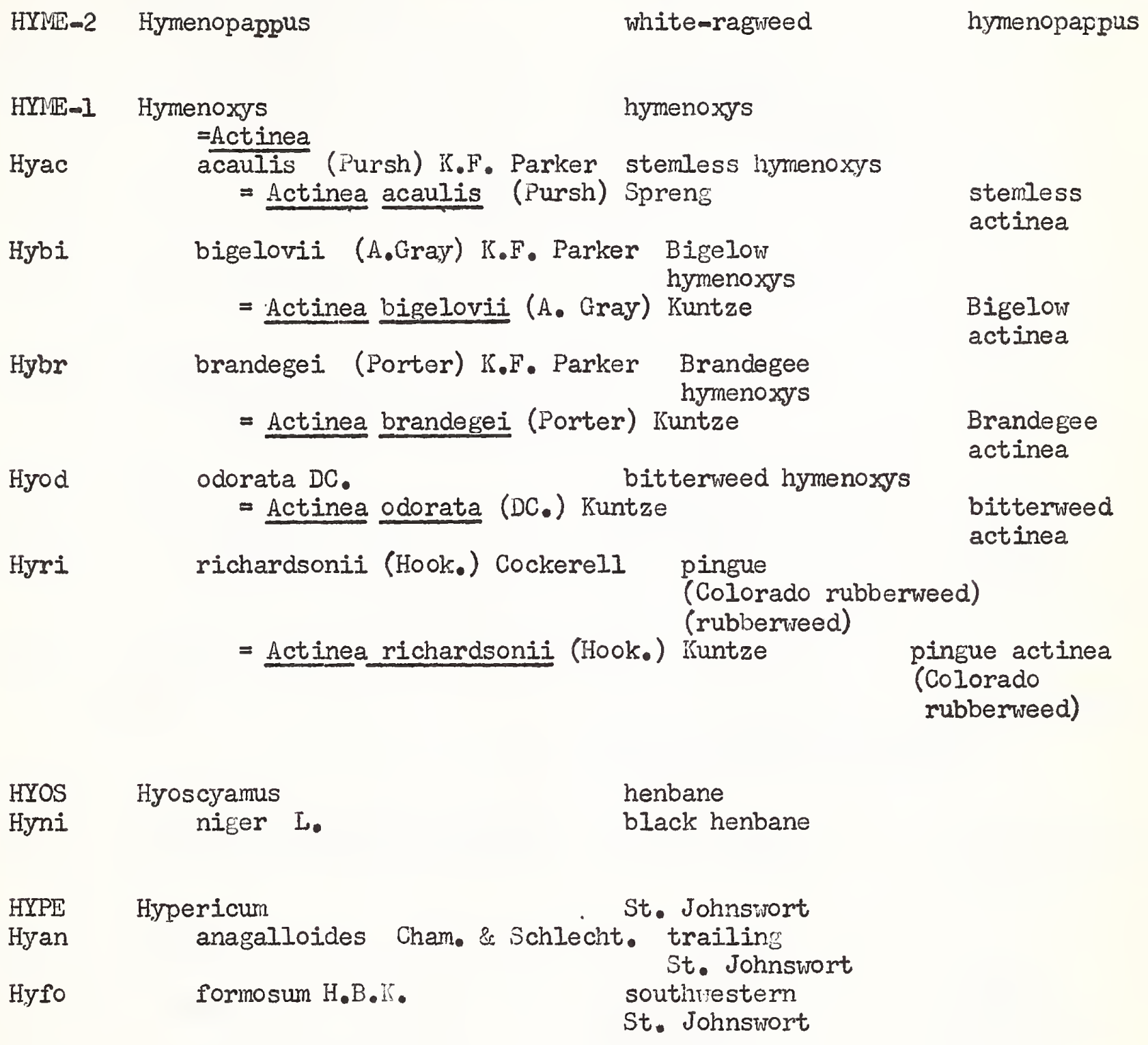

Hypopitys See Ionotropa

Ilgr Iliamna grandiflora (Rydb.) Wiguins wildhollyhock = Sphaeralcea grandiflora Rydb.
IPOM Ipomoea = Quamoclit momingglory
Ipco coccinea Lo redstar morningglor
Iple leptotoma Torr. Quamoclit coccinea $\left(L_{0}\right)$ Moench
morningglory
scarlet star- glory

41 


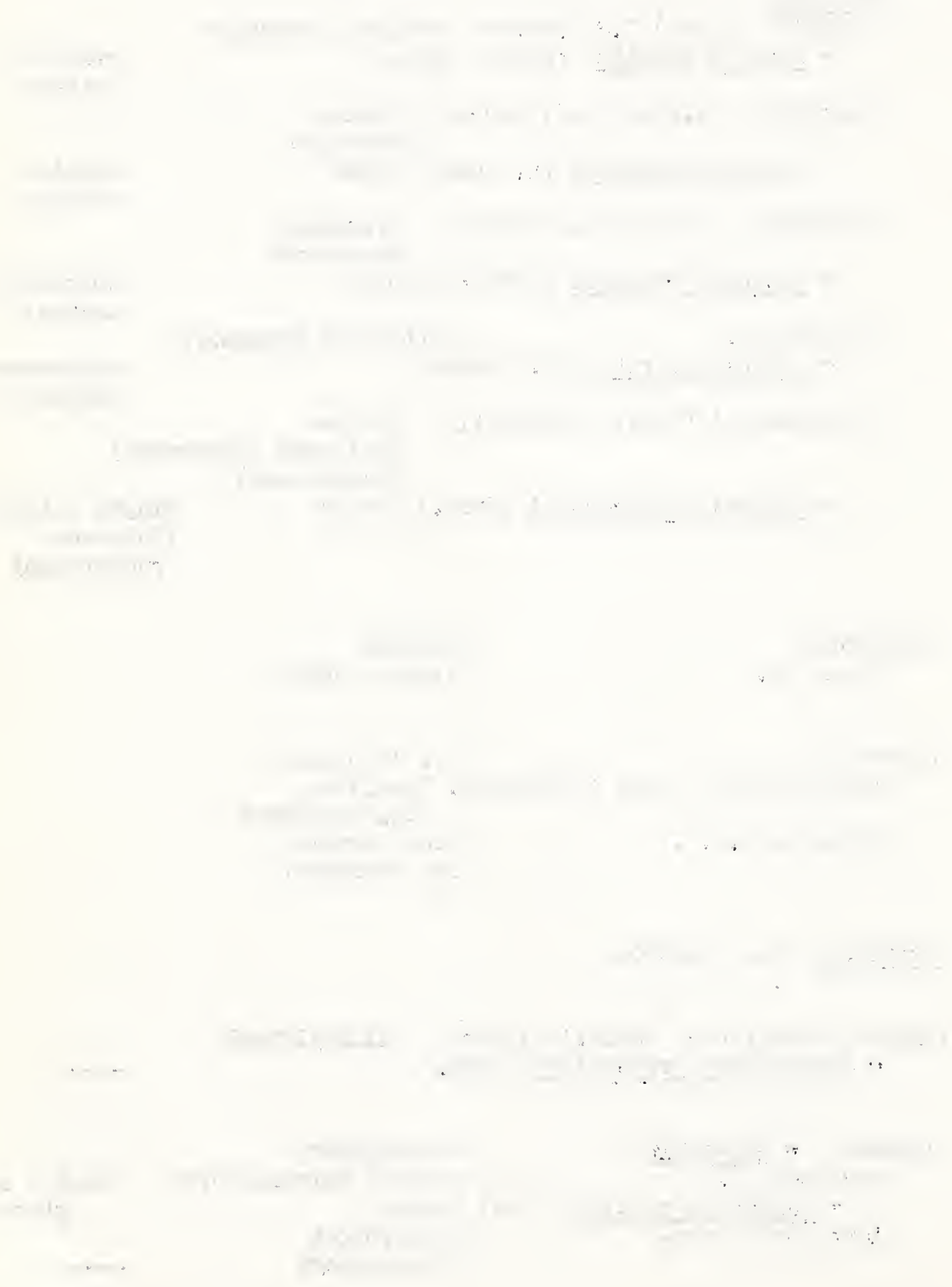




\section{Forbs List (continued)}

Ipomoea (continued)

Iplo

Ipth

Irhe

Irmi Iris missouriensis Nutt.

Isbr

IVA Iva

Ivax

Ivxa

Jure

Jussiaea repens I.

Kagr Kallstroemia grandiflora Torr.

KOCH Kochia

iroam

Koamv

Kosc

axillaris Pursh

xanthifolia Mutt.

scoparia (L.) Schrad. thus brachiatus (L.) B.S.P. = Trichostema brachiatum L.

bluecurls

marsh-elder

poverty marsh-elder

rag marsh-elder

yellowwaterweed

NOTE: Vaterweed is accepted common name for Anacharis.

Arizona-poppy

mock-cypress

greenmoliy

(redsage)

hairy greenmolly

$$
=\underline{K}_{0} \text { vestita (S.Wats.) Rydb. }
$$

sumner-cypress

dwarf-dandelion

dwarf-dandelion
Arizona-poppy caltrop \%

summercypress greenmolly summercypress gray sumner-cypress

Belvedere summer-cypress
KRIG Krigia

$\mathrm{Krbi}$ biflora (Nalt.) Blake

\% Suggested cominon name 


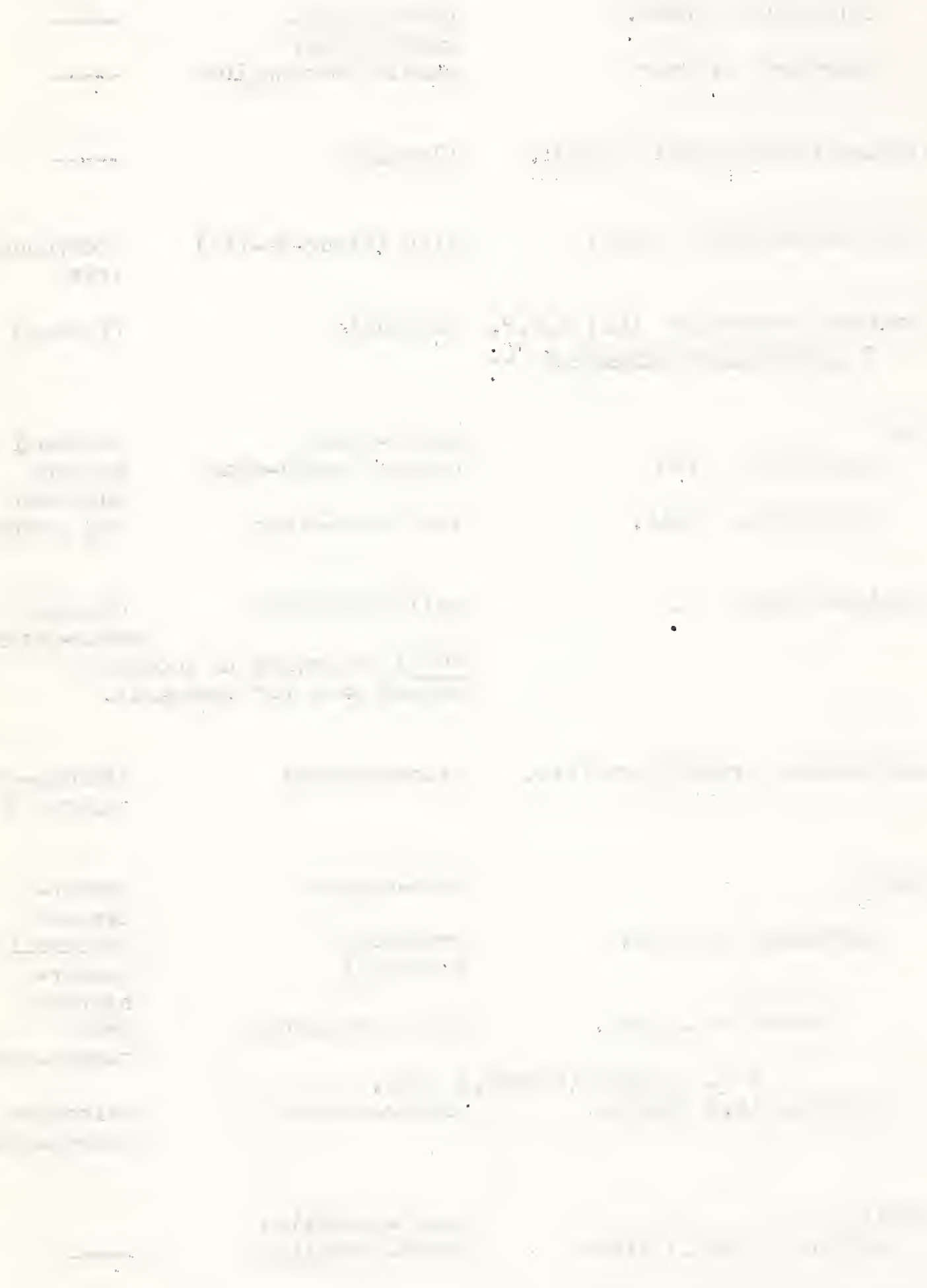


Forbs List (continued)

Lacinaria See Liatris

LACT Lactuca

Lapu pulchella (Pursh) DC.

Lase serriola L.

LAPP Iappula

LATH

Laar

Laeu

Lagr

Lala

Lathyrus arizonicus Britt. eucosmus Butters \& St. John graminifolius (S. Wats.) Vhite laetivirens Greene NOTE: Lathyrus to Lathyrus leucanthus Rydb., and perhaps not distinct from it; aspen peavine is the SPI common name for Lathyrus leucanthus.

peavine

Arizona peavine

bush peavine

grassleaf peavine

aspen peavine lettuce (wild lettuce) poison lettuce chicory lettuce NOTE: If any lettuces are actually "Poison", the name had best be reserved for Lactuca virosa. prickly lettuce

stickseed

$\rightarrow \infty-\infty$

Lavauxia See Oenothera

LAYI Layia

Lagl

glandulosa

(Hook•) Hook. \&e

tidytips

whitedaisy tidytips

Leontodon See Tarasacum

LEPI Lepidium See also TRES AND SHRUBS pepperweed LIST

Ledr draba I. = Cardaria draba (L०) Desv.

Lepe perfoliatum I.

hoarycress

pepperweed

clasping pepperweed

Leptilon canadense See Erigeron canadensis

LESQ

Lesquerella

bladderpod

Lemo Leucocrinum montanum Nutt.

starlily

common starlily

LEWI Lewisia

bitterroot

lewisia 


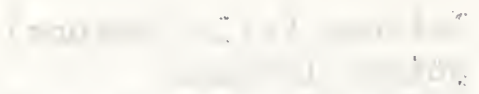

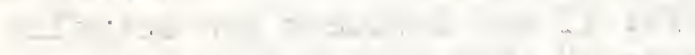

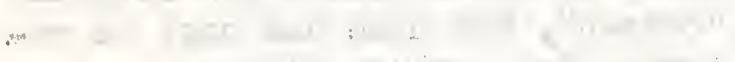

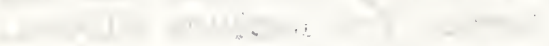

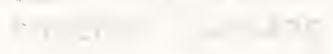

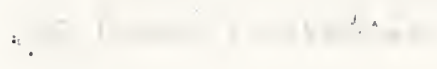

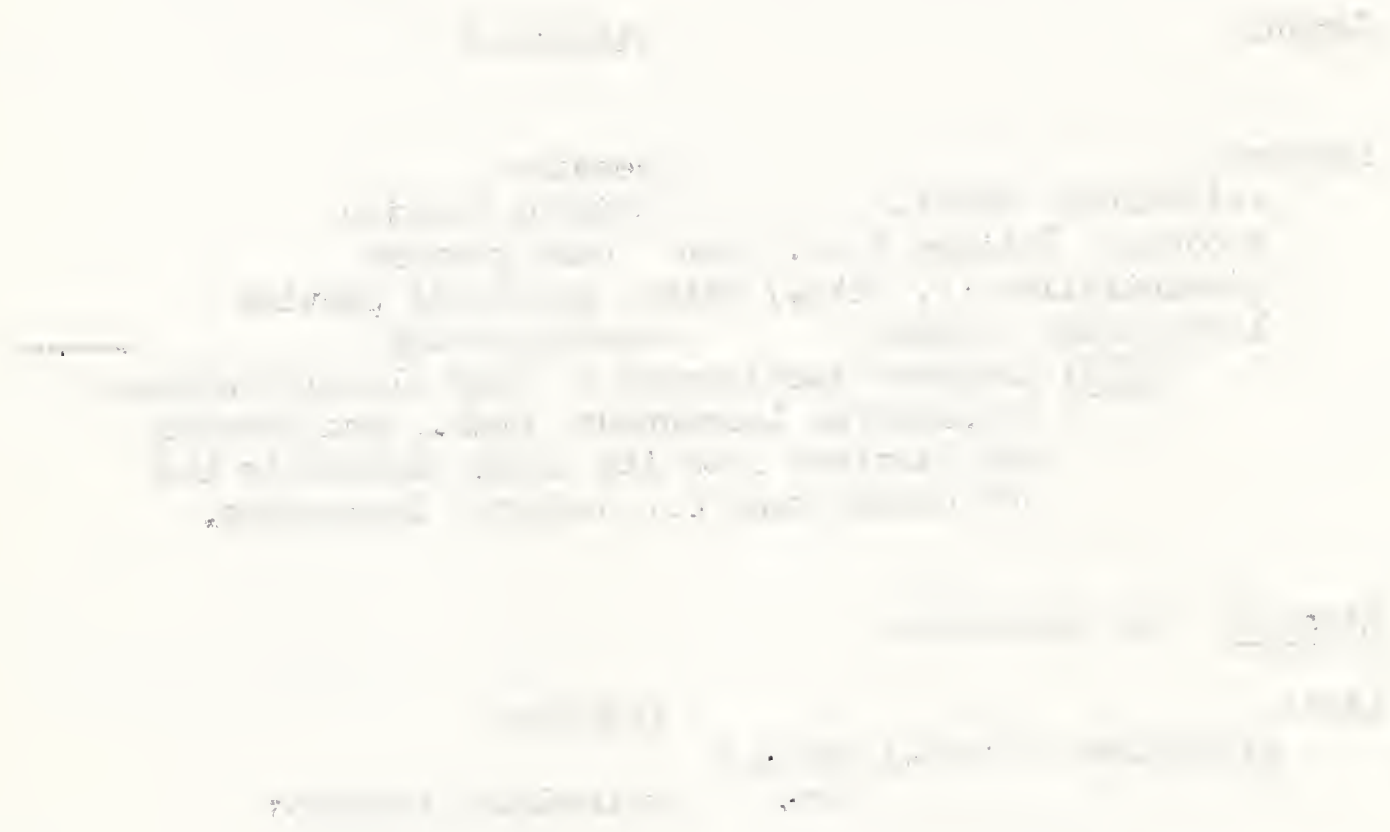

in

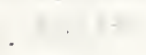

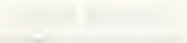

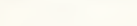

i, $1+1+2$

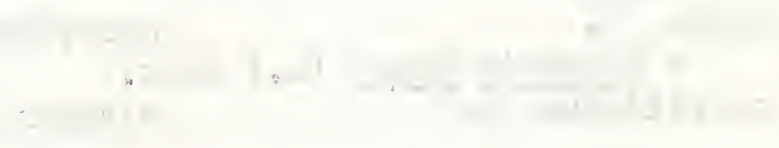

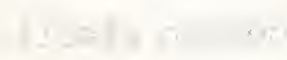

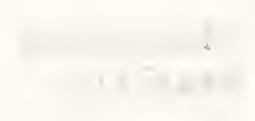

$1,-=$

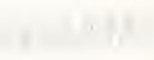

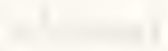


Forbs List (continued)

$\begin{array}{rlrl}\text { Lipu Liatris punctata Hook. } & \text { blazing-star } & \begin{array}{c}\text { dotted } \\ \text { gayfeather }\end{array} \\ = & \text { Lacinaria punctata (Hook.) } \begin{array}{l}\text { Kuntze } \\ \text { NOTE: Blazing-star is accepted }\end{array}\end{array}$

Lipo Ligusticum porteri Coult. \& Rose

loveroot:

(chuchupate)

Porter

Iigusticum

LIII
Lipa
Lium

Lilium

parryi S. Tats

umbellatum Pursh
IiIy

lemon lily

orangecup lily
Parry lily western orangecup lily

\begin{tabular}{|c|c|c|}
\hline $\begin{array}{l}\text { LINA }=1 \\
\text { Lian }\end{array}$ & $\begin{aligned} & \text { Linanthus }=\text { Gilia, in part } \\
& \text { androsaceus (Steud.) Greene } \\
& \text { = Gilia androsacea Steud }\end{aligned}$ & trumpet gilia \\
\hline Liau & $\begin{array}{l}\text { aureus (Nutt.) Greene } \\
=\text { Gilia aurea Nutt. }\end{array}$ & yellow trumpet \\
\hline Libi & $\begin{array}{l}\text { bigelovii (A Gray) Greene } \\
\quad \approx \text { Gilia bigelovii A. GraJ }\end{array}$ & $\begin{array}{l}\text { painted trumpet } \\
\text { ay }\end{array}$ \\
\hline Lide & $\begin{array}{l}\text { demissus (A.Gray) Greene } \\
=\text { Gilia demissa A. Gray }\end{array}$ & bell trumpet \\
\hline Lidi & $\begin{array}{l}\text { dichotomus Benth. } \\
=\text { Gilia dichotoma Benth. }\end{array}$ & evening-snow \\
\hline LINA $\sim 2$ & Linaria & toadflax \\
\hline & canadensis (L.) Du liont & bigflover toadfla \\
\hline
\end{tabular}

yellow gilia

$\rightarrow \infty \infty \infty$

$-\infty \infty$

evening-snow gilia

Libo Linnaea borealis L. twinflower

Iiboa americana (Forbes) Rehder $\ldots$

American twinflower

LINU Linum flax

Lico Listera convallarioides (Swartz) Nutt. twayblade

INOTE: Twayblade is the SPN

common name for the genus Liparis

LITH-I Lithophragma

Lite tenella Nutt. woodland-star

slender voodland-star 


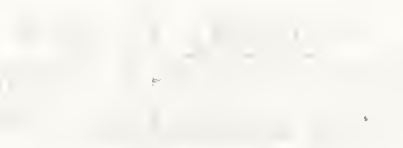

$\because$ 
Fiorbs List (continued)

LITH-2 Lithospermum

LOBE Lobelia

Loan

Loca

Locag

stoneseed (gromwell) gromwell

lobelia

bluebell lobelia

$\infty \rightarrow \infty$

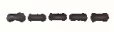

cardinalfloiver

gruina See Lobelia anatina

= L. gruina of U.S.

cardinalis $\mathrm{L}$.

Ssp. graminea (Lam。)

ic Vaugh western cardinalflower

$=$ L. Splendens of authors, NOT Villd. solendens See $\mathrm{L}$. cardinalis graminea

LOIIA Lomatium

biscuitroot

(Indianroot)

lomatium

NOTE: Biscuitroot is used for species with bulblike edible corms; lomatium is used for those with taproots.

LOTU Lotus

deervetch

(deerclover)

deervetch

Loco

Lopu

corniculatus I.

birdsfoot deervetch purshianus (Benth.) Clements ic Clements Spanish-clover

Lori

Lowr rigidus (Benth.) Greene desert deervetch wrightii (A. Gray) Greene

red-and-yellow-pea

shrubby deervetch Wright deervetch

Iupine

lupine (wild-pea,

blue-pea, blue-bean)

Luar-1

argenteus Pursh

Luar-2

arizonicus $S_{\text {. Tats. }}$ barbiger See I. sericeus

Lubr

Luco

Luhi brevicaulis $\mathrm{S}$. Wats concinnus Agardh hillii Greene

silvery lupine

Arizona lupine

shortstem lupine

bajada lupine

Hills lupine

NOTE: Named in honor of lir. Robert Hill of U.S. Forest Service, who collected the type specimen on the Coconino N.F. in 1911.

Luod odoratus Heller
Lupa-2 Wohave lupine

Palmer lupine 


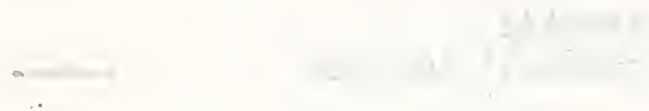

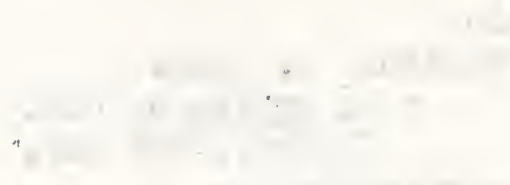

$$
\begin{aligned}
& \text { n }
\end{aligned}
$$

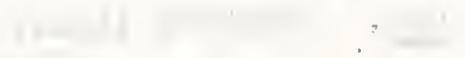

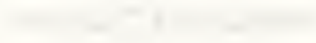
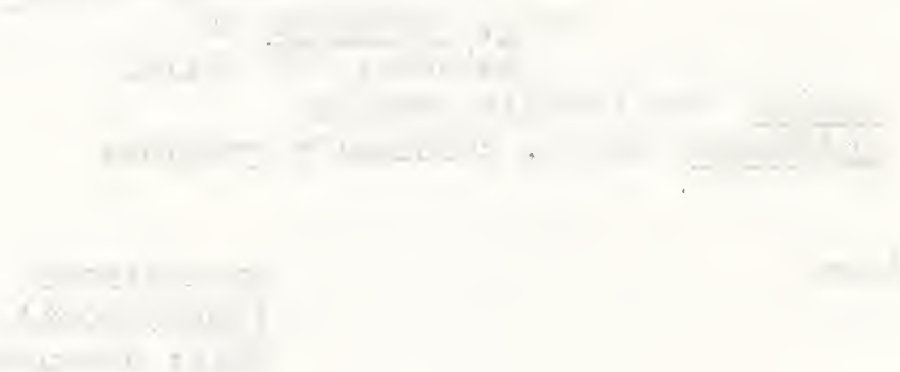

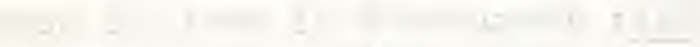

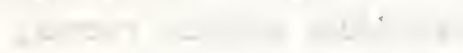

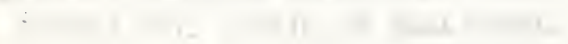

$$
x^{-2}+x^{-2}+y^{1}
$$

.
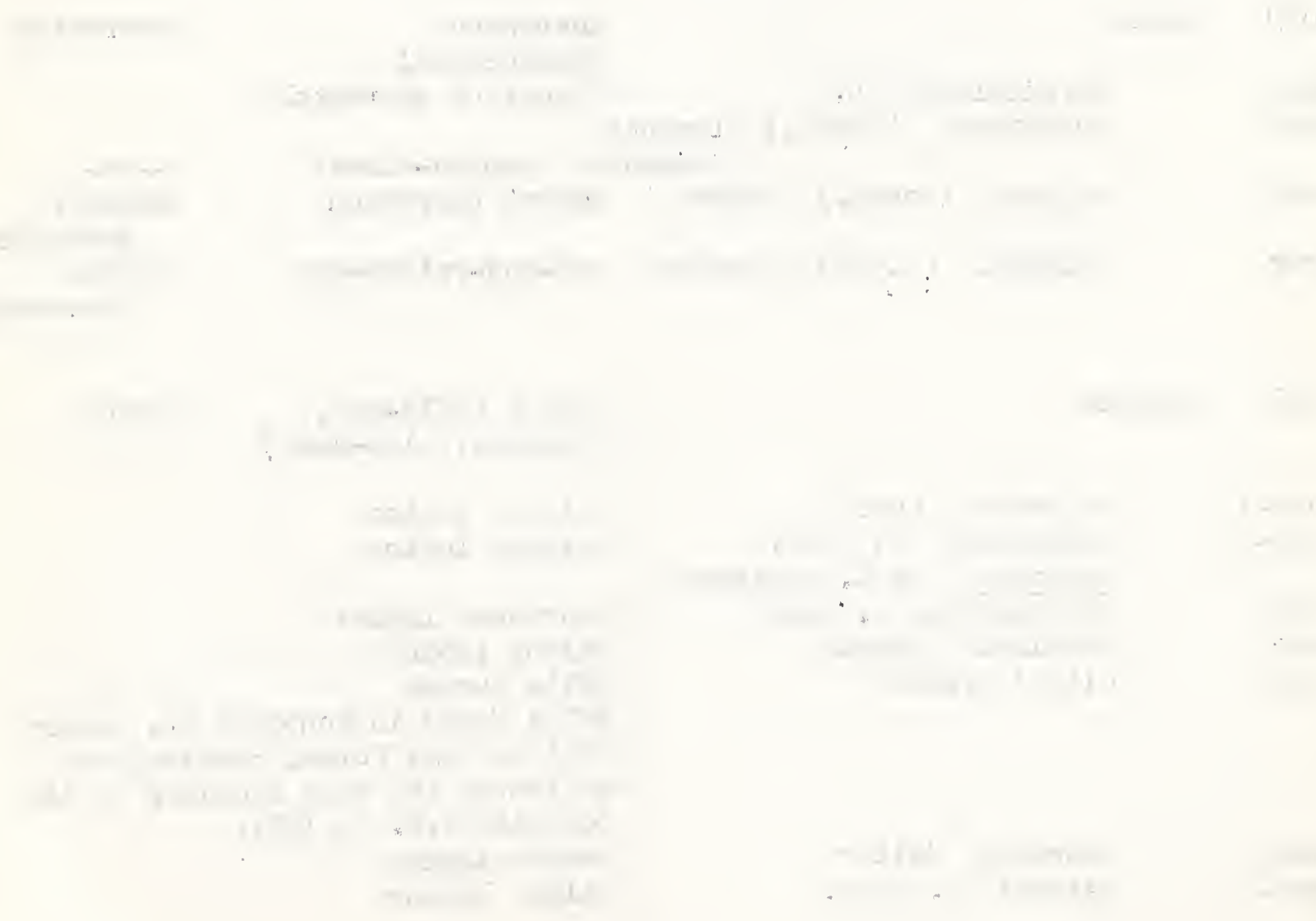
Forbs List (continued)

Lupinus (contimued)

Isupu

Luru

Luse

Lush

Lusp

Lusu

Lydr Lychnis drummondii (Hook,) S. Jats. campion

Lyco Lycopus

Lylu

LYGO

Lysp

Lygodesmia spinosa liutt.

LYSI-I Lysimachia

Lyci lupine (wild-pea, blue-pea, blue-bean)

rusty Iupine

yellnweye lupine

silky lupine

desert luoine

Coviter lupine

arroya Iupine
Drummond

campion

bugleweed

bugleweed

skeletonplant

thorn skeletonplant

loosestrife

fryced loosestrife

= Steironema ciliatum (I.) Raf. fringed

steironema

Navi Macromeria viridiflora DC. = Horrori (A. Gray) liack.

= Onosmujum thurberi A. Gray

marbleseed

MADI Madja

Iagl-1 glomerata Hook.

tarweed

cluster tarweed

MALA Malacothrix

Placo

Magl-2

coulteri A. Gray

glabrata A. Gray

snakes-head

desertdandelion

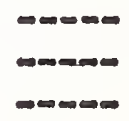


1

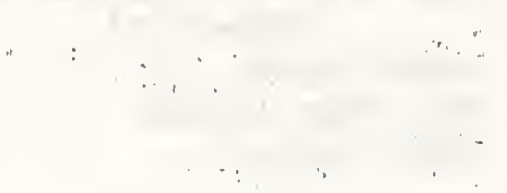

$4 \quad, \quad \ldots$

$\because$,

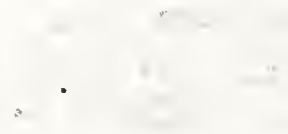


Forbs List (continued)

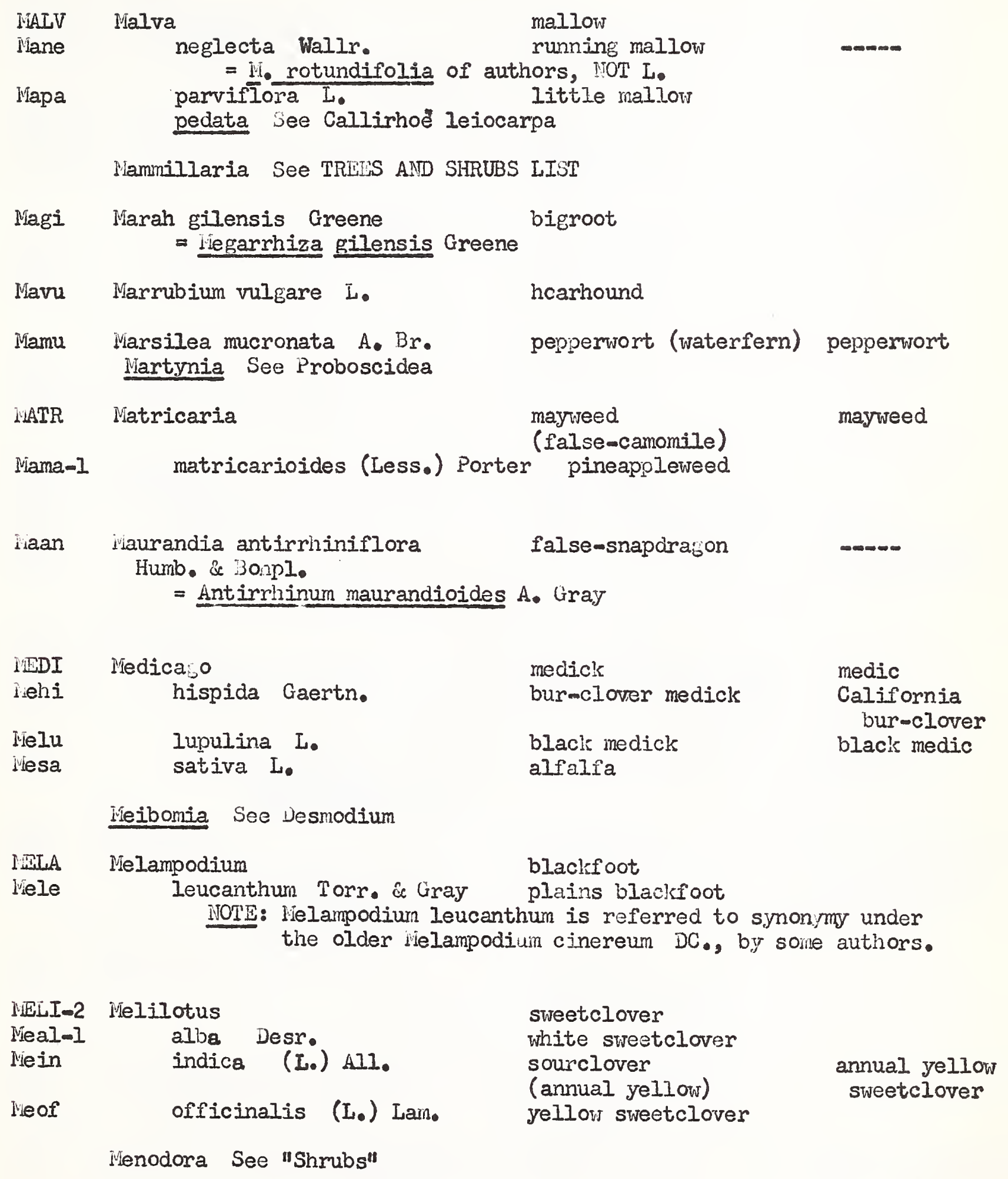


... . .

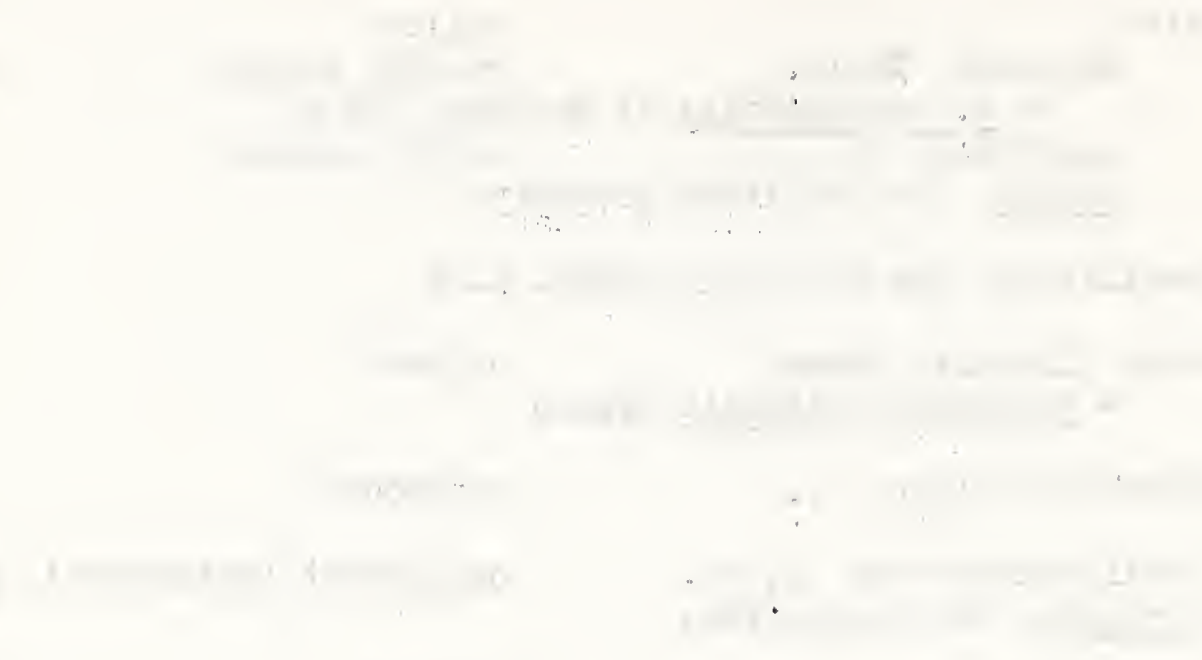

$11+20$
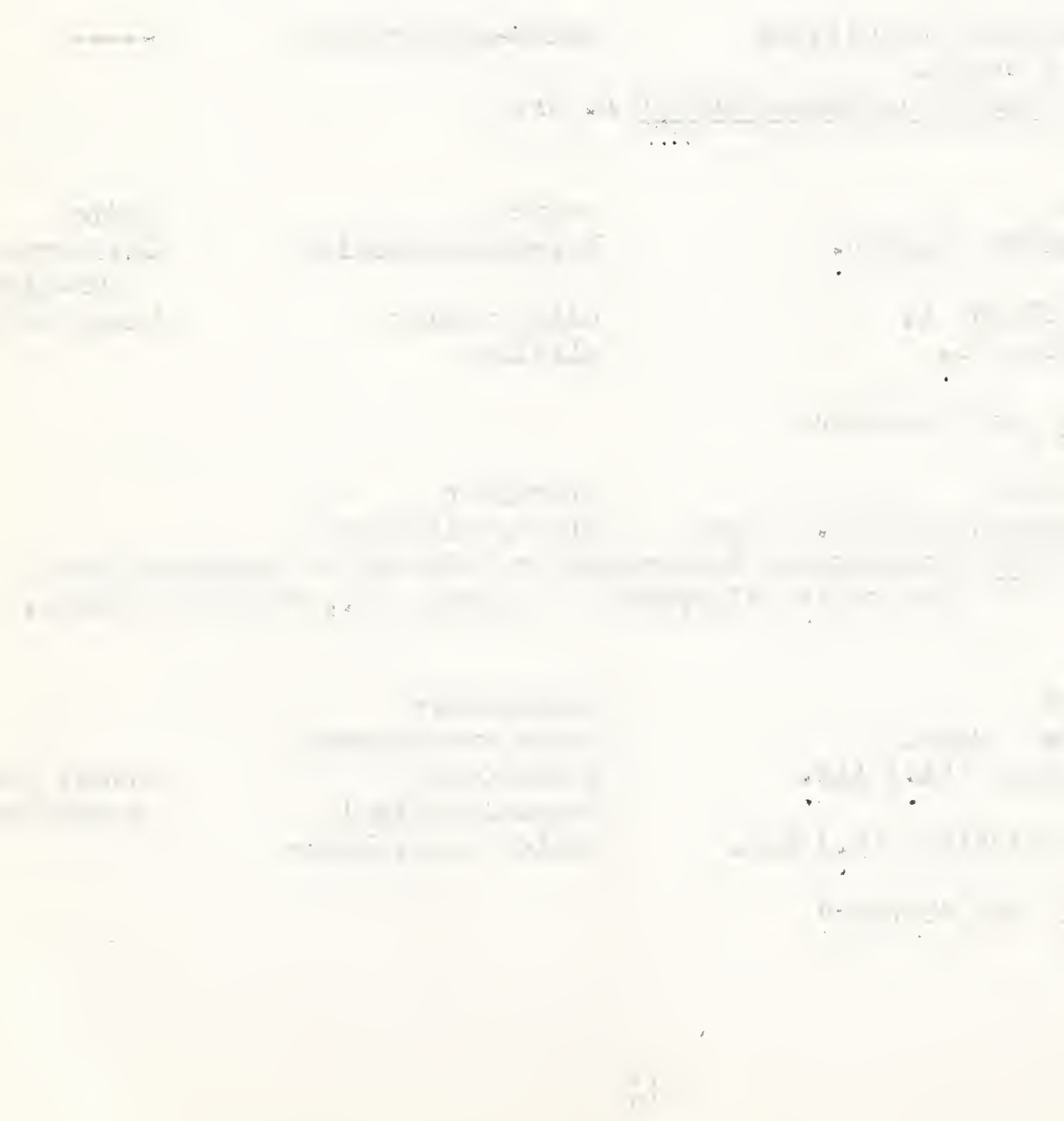


\section{Forbs List (continued)}

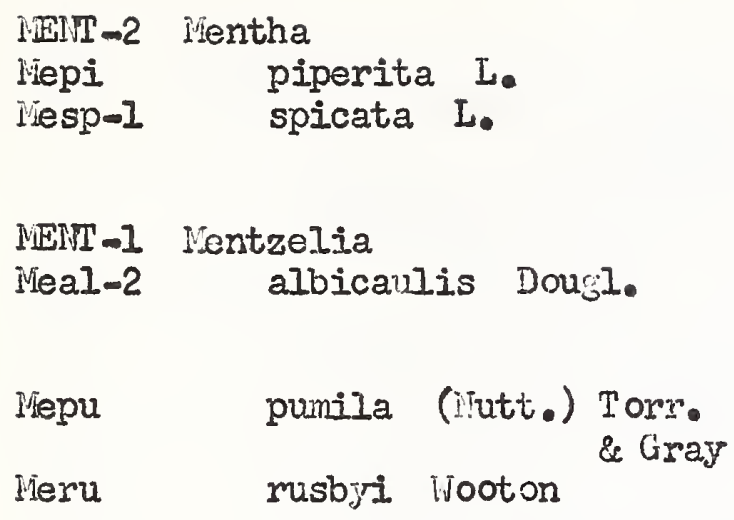

Minu multiflora (Torr.) A. Gray $\rightarrow$ Quamoclidion multiflorum Torr.

MOLD ILldavica

Niopa parviflora (ilutt.) Britt. - Dracocephalum parviflorum Nutt.

Move Mollugo verticillata I.

INONA Monarda

Wome menthaefolia Graham

Mope-2 pectinata liutt.

Monu Monolepis nuttalliana (Schult.) patata (patota) mint

peppermint

spearmint

$\begin{array}{lc}\text { blazing-star } & \text { mentzelia } \\ \text { (stickleaf) } & \text { whitestem } \\ \text { whitestern blazing-star } & \text { mentzelia } \\ \text { golden blazing-star } & \text { - - - - } \\ \text { creamy glazing-star } & \text {-.-.-m }\end{array}$

bluebells

Miexican-star

monkeyflower

crims on monkey flower

yellow monkeyflower

primrose monkejflower

cornmon

monkeyslower

four - o' clock

weakstem four $-0^{\prime}$ clock longneck four-o' clock

wild four-o'clock

sweet fouro'clock

Colorado four-O'clock

dragonhead

dragonhead

false-dragonhead Arnerican falsedrags onhead

carpetweed (Indian-chickweed)

beebalm (horsemint) mintleaf beebalm pony beebalm Greene
Nuttall monolepis 


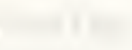

,

$+1$

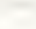
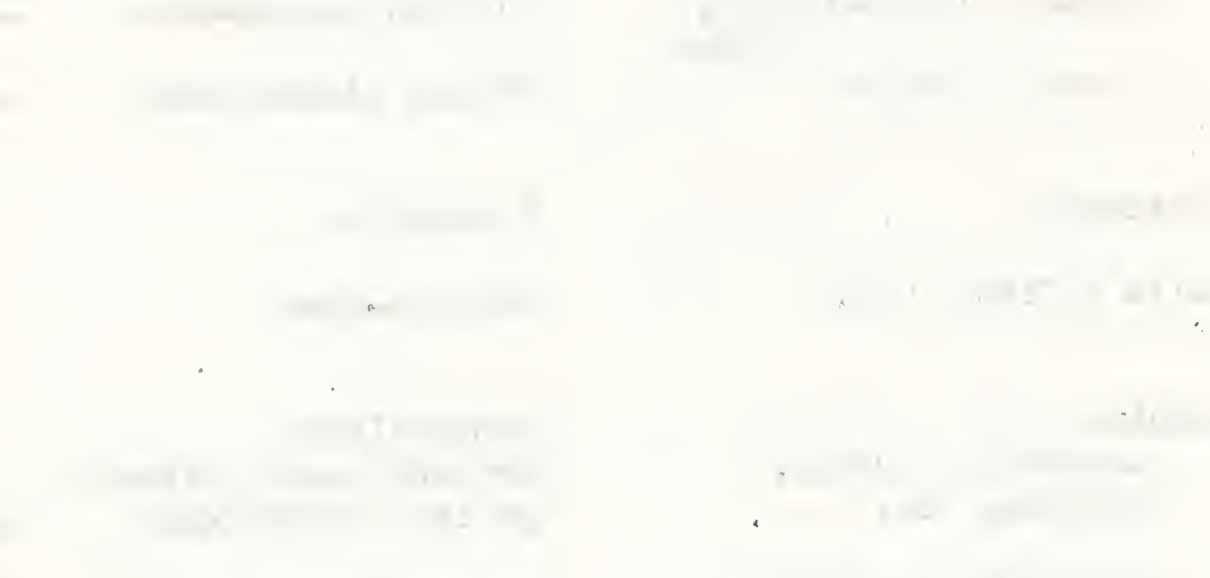

-

$;$

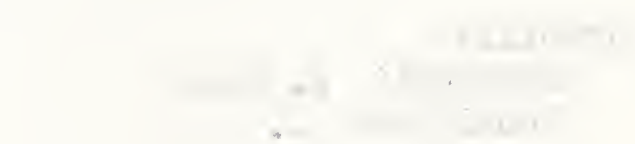

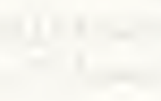

,

$*$

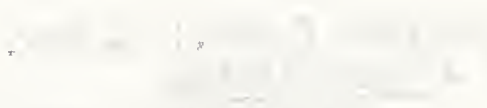

.th

a n

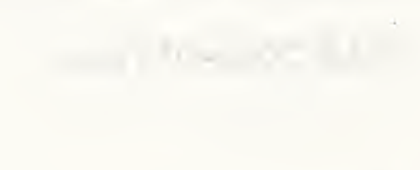

n.?

.
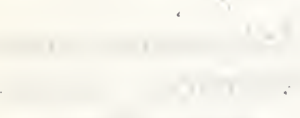

(1)

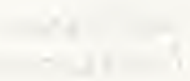
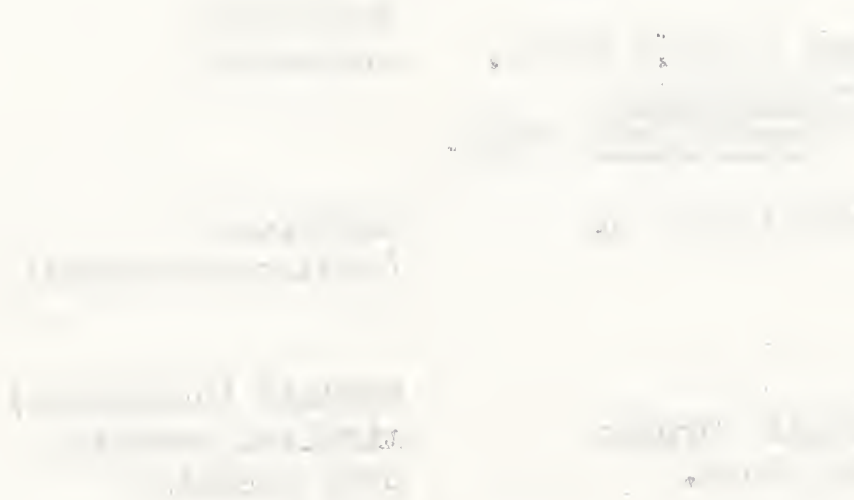


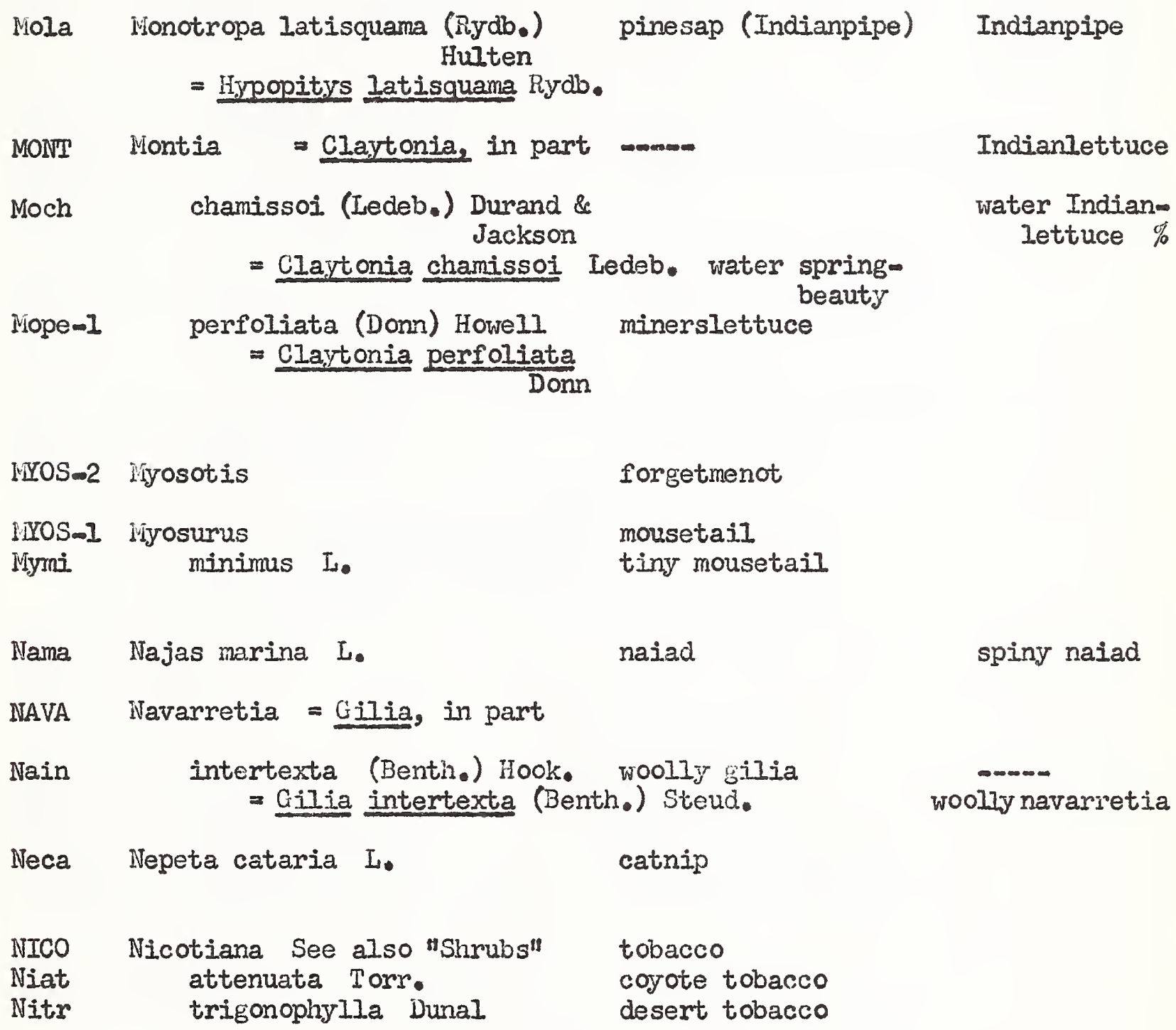

IHOS-2 Myosotis

MOS-I DiYosurus

Mymi minimus $I_{\text {. }}$

Nama Najas marina L.

NAVA Navarretia = Gilia, in part

Nain intertexta (Benth.) Hook. woolly gilia $=$ Gilia intertexta (Benth.) Steud.

Neca Nepeta cataria L. catnip

NICO

Niat

Nitr

naiad

tiny mousetail

spiny naiad

Indianlettuce

water Indian-

lettuce \% 
Forbs List (continued)

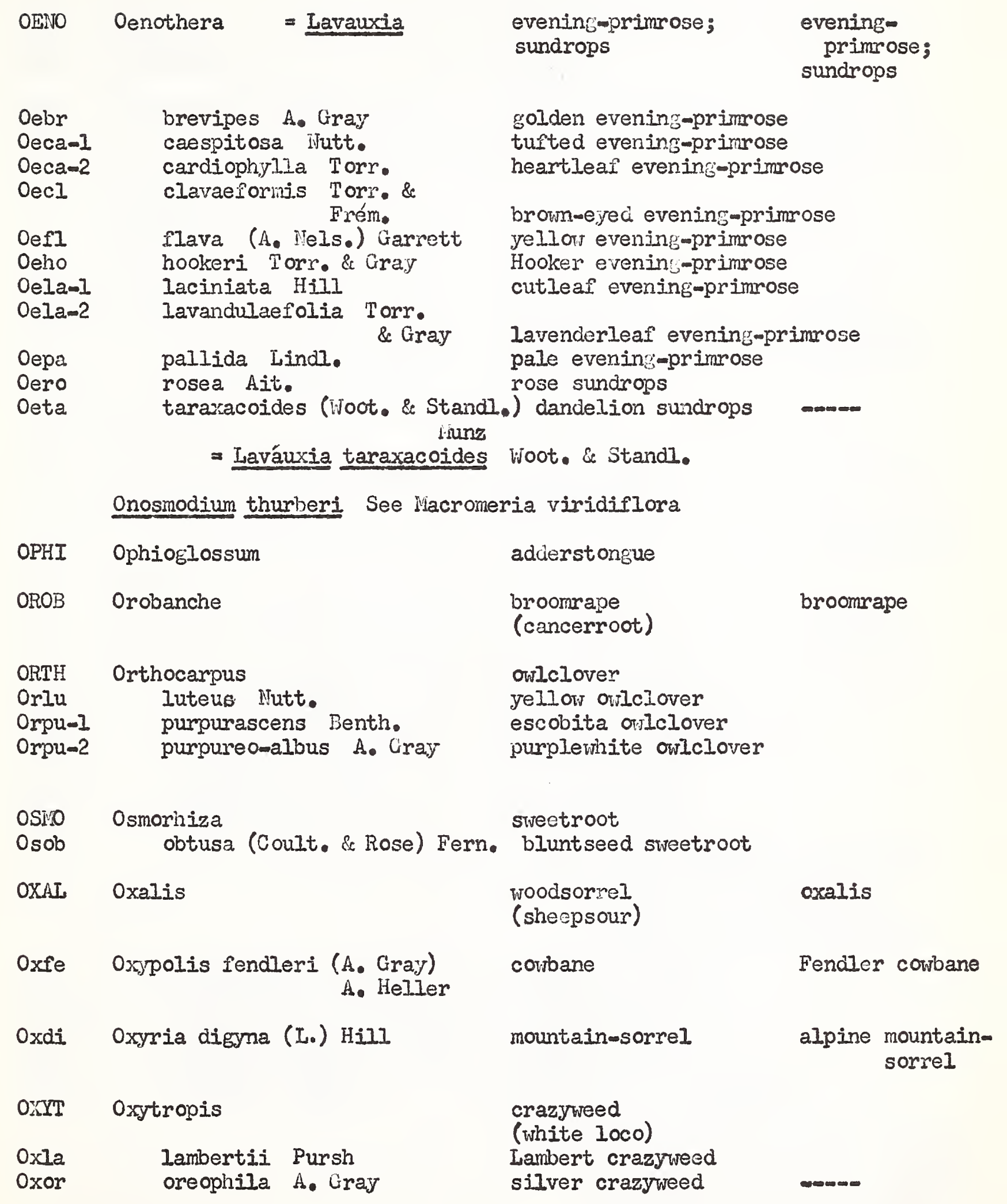




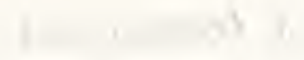

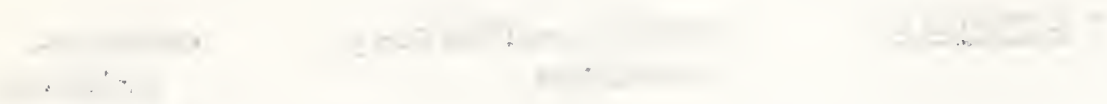

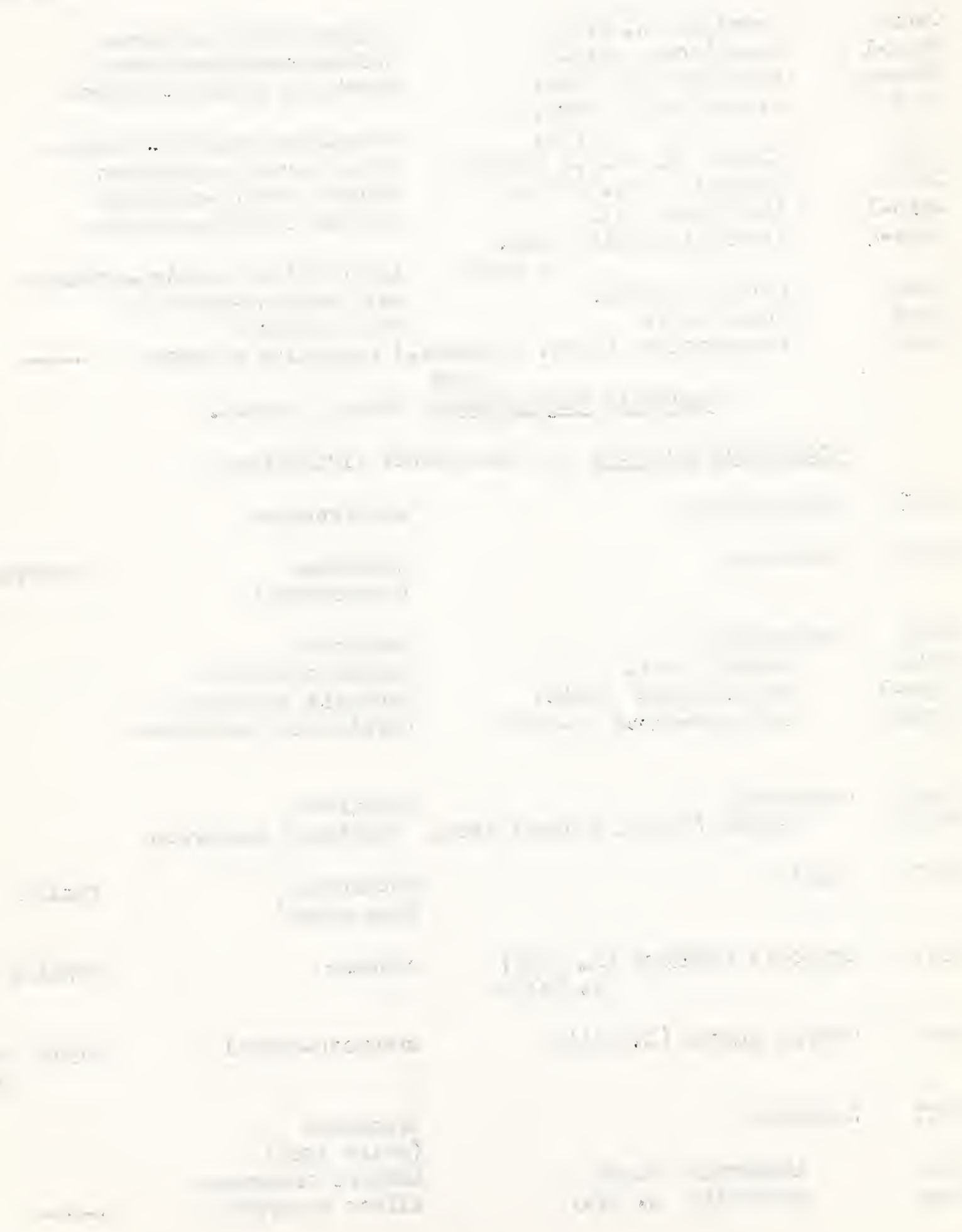


Forbs List (continued)

\begin{tabular}{|c|c|c|c|}
\hline PANA & Panax & ginseng & \\
\hline PARI & Parietaria & pellitory & \\
\hline Papa & Parnassia parviflora DC. & grassmof -parnassus & $\begin{array}{l}\text { smallflower } \\
\text { parnassia }\end{array}$ \\
\hline Paja & $\begin{array}{l}\text { Paronychia jamesii Torr. \& Gray } \\
\text { Parosela See Dalea }\end{array}$ & nailwort & James nailwort \\
\hline Pasa & Pastinaca sativa I. & parsnip & garden parsnip \\
\hline $\begin{array}{l}\text { PECT-I } \\
\text { Pefi }\end{array}$ & $\begin{array}{l}\text { Pectis } \\
\text { filipes Harv. \& Gray } \\
\text { papposa Harv. \& Gray }\end{array}$ & $\begin{array}{l}\text { fetidmarigold } \\
\text { threadstem } \\
\text { fetidmarigold } \\
\text { chinchweed }\end{array}$ & $\begin{array}{l}\text { pectis } \\
\text { threadstem } \\
\text { pectis }\end{array}$ \\
\hline PECT -2 & Pectocarya & combseed & \\
\hline PEDI & Pedicularis & $\begin{array}{l}\text { woodbetony } \\
\text { (fernleaf) }\end{array}$ & pedicularis \\
\hline Pegr-1 & groenlandica Retz. & elephanthead & $\begin{array}{l}\text { elephanthead } \\
\text { podicularis }\end{array}$ \\
\hline PELC & Pellaea & clifflorake & \\
\hline PENS & Penstemon See also "Shrubs" & $\begin{array}{l}\text { penstemon } \\
\text { (beard tongue) }\end{array}$ & penstemon \\
\hline $\begin{array}{l}\text { Peam } \\
\text { Pean-1 } \\
\text { Peba } \\
\text { Pebr } \\
\text { Peca-2 } \\
\text { Peco } \\
\text { Pela }\end{array}$ & $\begin{array}{l}\text { ambiguus Torr. } \\
\text { angustifolius Nutt. } \\
\text { barbatus (Cav.) Roth } \\
\text { bridgesii A. Gray } \\
\text { caespitosus Nutt. } \\
\text { comarrhenus A. Gray } \\
\text { laevis Penneli }\end{array}$ & $\begin{array}{l}\text { gilia penstemon } \\
\text { narrowleaf penstemon } \\
\text { beardlip penstemon } \\
\text { Bridges penstemon } \\
\text { mat penstenon } \\
\text { dusty penstemon } \\
\text { royal penstemon }\end{array}$ & \\
\hline $\begin{array}{l}\text { Peli } \\
\text { Peol } \\
\text { Pepa-2 } \\
\text { Peps } \\
\text { Pest } \\
\text { Pesu-1 }\end{array}$ & $\begin{array}{l}\text { linarioides A. Gray } \\
\text { oliganthus Woot. \& Standl. } \\
\text { pachyphyllus A. Gray } \\
\text { pseudospectabilis Jones } \\
\text { strictus Benth. } \\
\text { subulatus Jones }\end{array}$ & $\begin{array}{l}\text { NOTI: Royal penstemon } \\
\text { comnon name for } \\
\text { speciosus Dougl } \\
\text { authors regard } \mathrm{P} \\
\text { a synonym of } P . \\
\text { toadflax penstemon } \\
\text { Wooton penstemon } \\
\text { thickleaf penstemon } \\
\text { desert penstemon } \\
\text { Rockyi ountain penstemon } \\
\text { scarletbugler penstemon } \\
\text { NorE: Scarletbugler pen } \\
\text { SPN cornon name f } \\
\text { thilolius, a Cali } \\
\text { species. Scarlet } \\
\text { is suggested for } \\
\text { species. }\end{array}$ & $\begin{array}{l}\text { is the SPN } \\
\text { Penstemon } \\
\text { o some } \\
\text { speciosus. } \\
\text { laevis as } \\
\text { stemon is the } \\
\text { or P. centran- } \\
\text { fornia coastal } \\
\text { tube pensterion } \\
\text { this Arizona }\end{array}$ \\
\hline
\end{tabular}




\section{$1+\cdots 1-$}

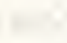

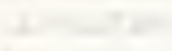

r.

$\cdot$

$+2$

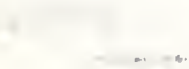

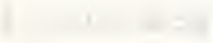

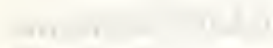

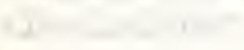

1. .. 1 , ,

$$
\begin{aligned}
& \text { a , + } \\
& \xi^{-7} \leq+\cdots \\
& \text { is } \\
& \text { mo }+1, \therefore \quad= \\
& ,+2=1,10
\end{aligned}
$$

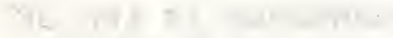

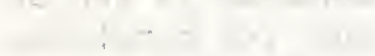

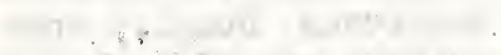

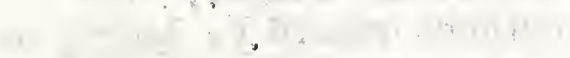

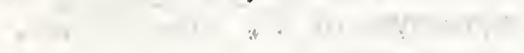

$$
\begin{aligned}
& +2+2+\infty+\infty
\end{aligned}
$$

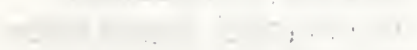$$
\because+12+2+20
$$

(5)

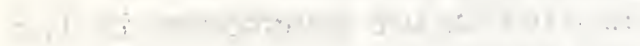

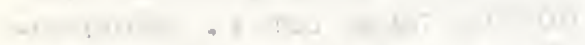

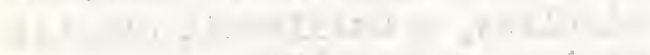

Con

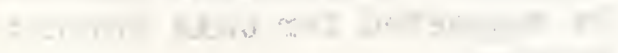

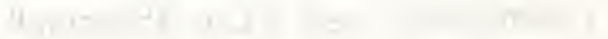
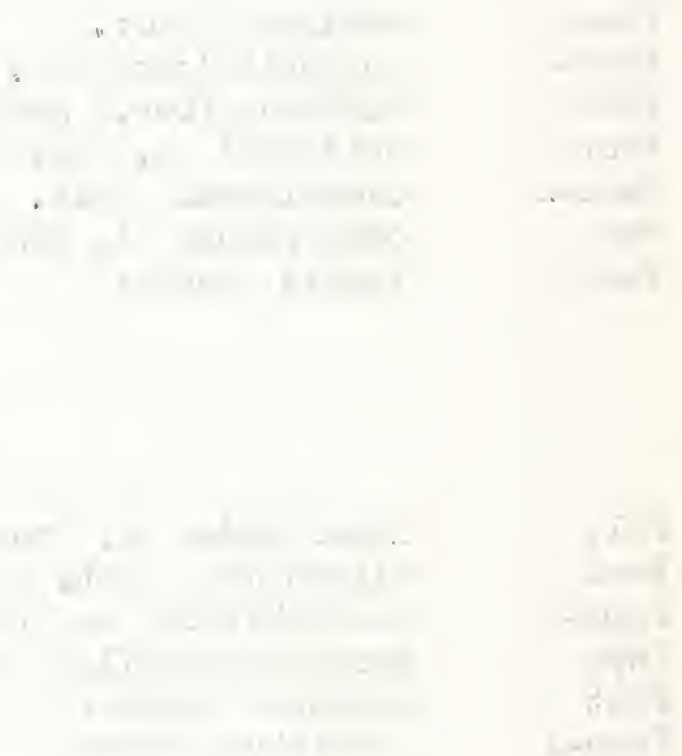

$$
\text { a. }+2=
$$


Forbs (continued)

Penstemon (continued)

Pesum2

Peut

Pevi superbus A. Hels. utahensis Eastw. virgatus A. Gray pensternon

Santa Rita penstemon

Utah penstemon

wandbloom penstemon

Peramium See Goodyera

PERE Perezia

Pena

nana A. Gray

desertholly

perezia

Pegra Perideridia gairdneri (Hook, \& wildcaraway
Arn. Wathias

= Carum gairdneri (Hook. \& Arn॰) A. Gray

yampa

PETA Petalostemon = Petalostemum prairieclover

NOTE; Petalostemon is conserved against Petalostemum.

Peca-1

Pecao

Pefl

Pepu

Pepa-3

$$
\begin{gathered}
\text { candidus (ijilld.) Iilchx. white prairieclover } \\
\text { oligophyllus (Torr.) Hermann slender white } \\
\text { prairieclover }
\end{gathered}
$$

NOTE: Plants of our area more properly referable to this variety.

flavescens S. Wats. purpureus (Vent.) Rydb.

Petunia parviflora Juss. yellow prairieclover purple prairieclover petunia
NOTE: mat petunia is

\begin{tabular}{|c|c|c|c|}
\hline PHAC & Phacelia & $\begin{array}{l}\text { phacelia } \\
\text { empillar-weed) }\end{array}$ & phacelia \\
\hline Phca-2 & campanularia A, Gray & harebell phacelia & \\
\hline Phhe & heterophylla Pursh & varileaf phacelia & \\
\hline Phmi-1 & minor (Harv.) Thell. & bluebell phacelia & \\
\hline Phra & ramosissima Dougl. & branching phocelia & \\
\hline Phse & sericea (Graharn) & & \\
\hline & A. Gray & silky phacelia & \\
\hline Phta & tanacetifolia Benth. & tansy phacelia & \\
\hline PHAS & Phaseolus & bean & \\
\hline Phac & acutifolius A. Gray & tepary bean & Texas bean \\
\hline Phac1 & latifolius Freeman & $-\infty-\infty$ & tepary bean \\
\hline $\begin{array}{l}\text { Phan } \\
\text { Phme }\end{array}$ & angustissimus A. Gray & slimleaf bean & \\
\hline & $\begin{array}{l}\text { metcalfei woot. \& Standl. } \\
\text { - } \text { - retusus Benth., NOT }\end{array}$ & $\begin{array}{l}\text { Wetcalf bean } \\
\text { Moench }\end{array}$ & \\
\hline & retusus See $P_{0}$ metcalfei & & \\
\hline
\end{tabular}
sugijested as comion name for this species. 


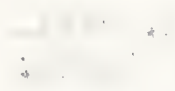

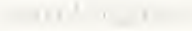

-
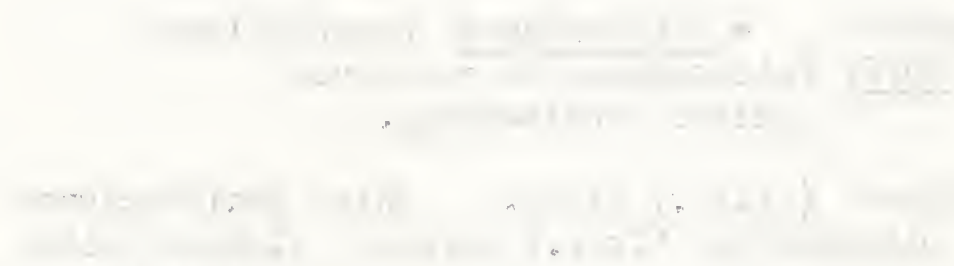
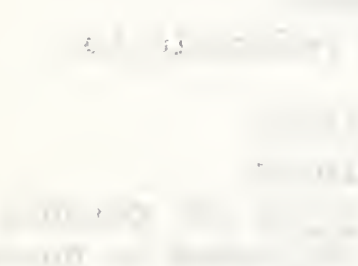

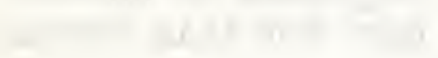

2.20

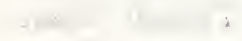

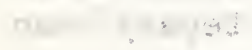

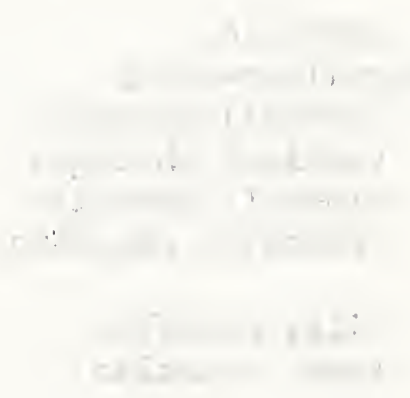

i

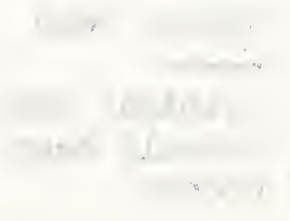

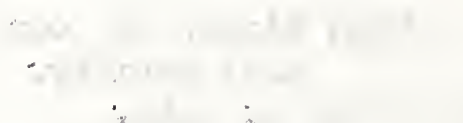

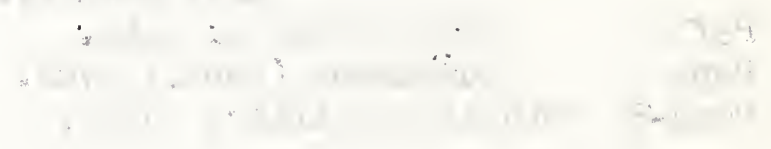

(1)
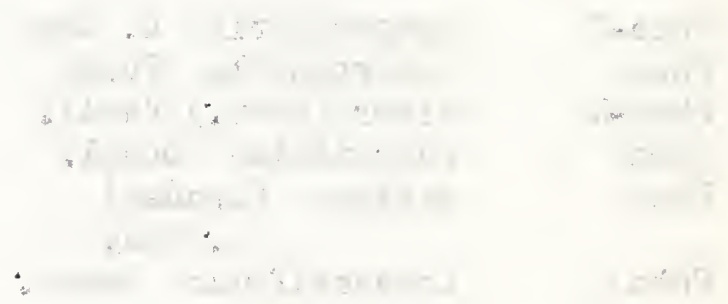

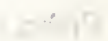

$1,1+\ldots$

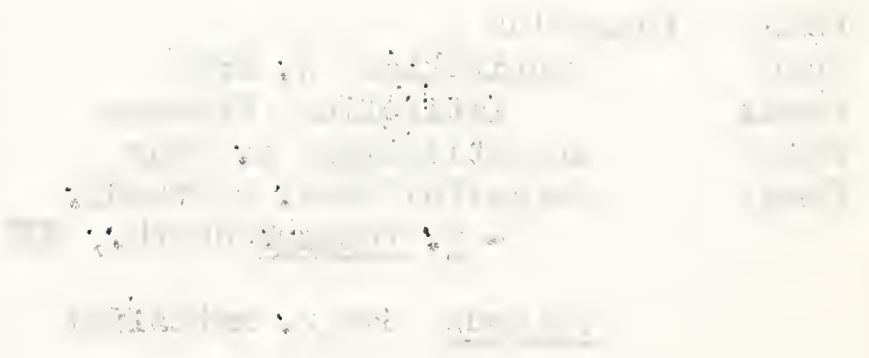


PHIL-1 Philibertia = Funastrurn

milkvine philibertia

NOTE: Milkvine is the accepted common name for Vincetoxicum.

$\begin{array}{lll}\text { PHLO } & \text { Phlox } & \text { phlox } \\ \text { Phau } & \text { austromontana Coville } & \text { desert phlox } \\ \text { Phca-3 } & \text { caespitosa Nutt. } & \text { tufted phlox } \\ \text { Phdi } & \text { diffusa Benth. } & \text { spreading phlox } \\ \text { Phho } & \text { hoodil Richards. } & \text { Hoods phlox } \\ \text { Phlo } & \text { longifolia Nutt. } & \text { longleaf phlox } \\ \text { Phna } & \text { nana Nutt. } & \text { Santa Fe phlox }\end{array}$

Phau-1 Pholistoma auritum (Lindl.) Iilja tomatoweed = Nemophila aurita Lindl.

purple nemophila

$\begin{array}{lll}\text { PHXS } & \text { Physalis } & \text { ground cherry } \\ \text { Phhe-I } & \text { heterophylla Nees } & \text { clanmy groundcherry } \\ \text { Phpu } & \text { pubescens I. } & \text { downy groundcherry }\end{array}$

PHYS-1 Physaria twinpod

$\begin{aligned} \text { Pham Phytolacca americana I. } & \text { pokeberry } \\ & \text { (poke, pigeonberry) }\end{aligned}$

Pitr Pityrogramma triangularis (Kaulf.)Maxon goldfern

PLAG Plagiobothrys bloodweed
(popcornflower) popcomflower

PIAN-I Plantago

Pler eriopoda Torr.

Plin insularis Eastw.

Plinf

fastigiata (Morris) Jeps. $=\underline{\text { P. }}$ fastigiata Morris

plantain--perennial species Indianwheat-- annual species redwool plantain desert Indianwheat $\underline{I}$

desert Indianwheat
PIla

PIma

PIpu

PIrh

Plvi lanceolata L. major L. purshii Roem \& Schult. rhodosperma Decne. virginica I. buckhorn plantain rippleseed plantain woolly Indianwheat redseed plantain paleseed plantain

l/Typical P. insularis not a plant of S. W. deserts but of $\mathrm{S}$. Calif. coastal islands. 


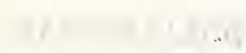

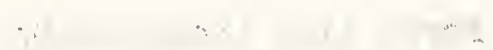

r Yr. r.

$\therefore+12+$
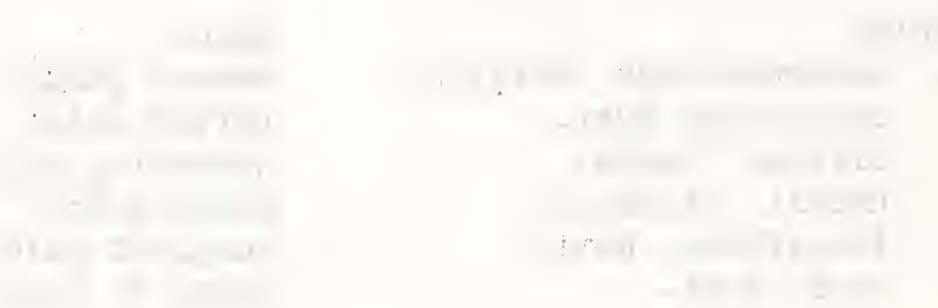

i

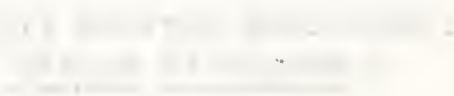


Forbs List (continued)

Plca-I Platystemon californicus Benth. creamcups

$\begin{array}{ll}\text { Plca-2 Pluchea camphorata (I.) DC. } & \text { camphor pluchea } \\ \text { (saltmarsh-fleabane) }\end{array}$

Poinsettia See Euphorbia

Potr-2 Polanisia trachysperma Torr. \& Gray clamyweed roughseed clammyweed

\begin{tabular}{|c|c|c|c|}
\hline $\begin{array}{l}\text { POLE } \\
\text { Pode }\end{array}$ & $\begin{array}{l}\text { Polemonium } \\
\text { delicatum Rydb. }\end{array}$ & $\begin{array}{l}\text { Jacobs-ladder } \\
\text { skunkleaf }\end{array}$ & polemonium \\
\hline & $=\underline{P}$. pulcherrimurn ssp. & delicatum (Rydb.) Brand & \\
\hline Pofi & filiciñum Greene & fernleaf Jacobs-ladder & $\begin{array}{l}\text { fernleaf } \\
\text { polemonium }\end{array}$ \\
\hline Pofl & flavum Greene & yellow Jacobs-ladder & yellow polemonium \\
\hline Povi & viscosum Nutt. & sticky Jacobs-ladder & sticky polemonium \\
\hline POLY-2 & Polygala. & milkwort & polygala \\
\hline Poac-2 & acanthoclada A. Gray & thorn milkwort & thorn polygala \\
\hline Poal & alba Nutt. & white milkwort & white polygala \\
\hline Posu & subspinosa S. Wats. & spiny miIkwort & spiny polygala \\
\hline POLY - 5 & Polygonatum & Solomonseal & \\
\hline Poco-4 & Cobrense (Woot. \& Standl.) & Gates Solomonseal & $\cdots$ \\
\hline POLY-3 & Polygonum & knotweed & \\
\hline Poco-3 & convolvulus L. & cornbind & dullseed cornbind \\
\hline Pope-1 & $\begin{array}{l}\text { persicaria L. } \\
\quad=\text { Persicaria mitis Gili }\end{array}$ & snartweed & spotted ladysthumb \\
\hline
\end{tabular}

POLY-4 Polystichum hollyfern

Pogr Porophyllum gracile Benth. yerba-del-venado

slender poreleaf

Pool Portulaca oleracea I. purslane (pursley) common purslane 

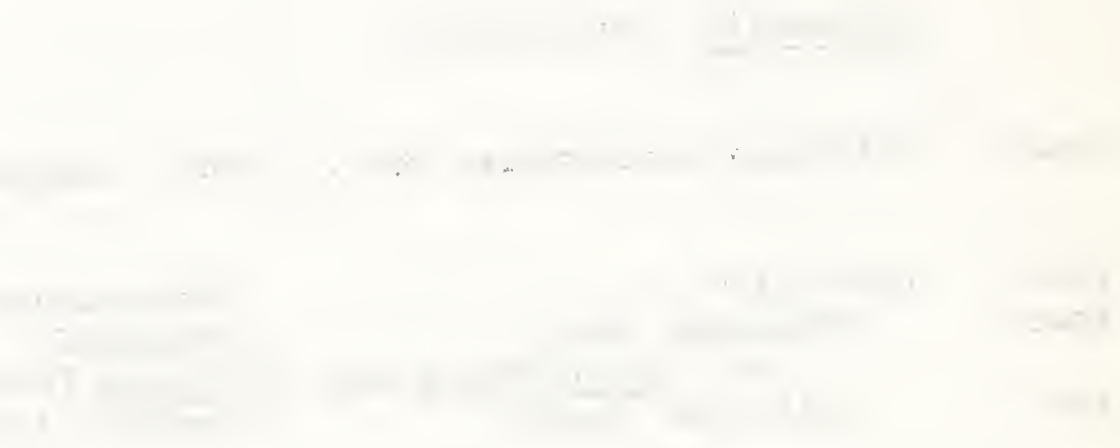

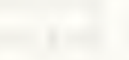
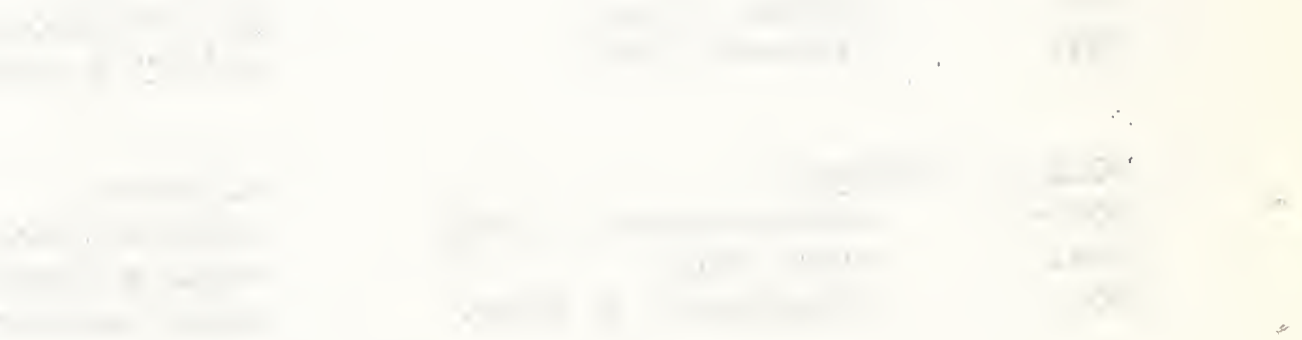

( 
Forbs List (continued)

POTA Potamogeton

pondweed

POTE Potentilla See also "Shrubs" cinquefoil

Poan-2 anserina I. silverweed cinquefoil

$=$ Argentina anserina (L.) Rydb.)

Pobi-2 biennis Greene biennial cinquefoil

$=$ P. millegrana $\mathrm{S}$. Wats. in part, NOT Engelm.

Poco-2 conciñna Richards. elegant cinquefoil

Podi diversifolia Lehm. varileaf cinquefoil

Pogl glandulosa Lindl. gland cinquefoil

Pohi hippiana Lehm. horse cinquefoil

Pono norvegica L. Norwegian cinquefoil

= $\underline{P}$. monspeliensis $I$.

Pope-2 pensylvanica I.

Popl plattensis Nutt.

Popu pulcherrima Lehm.

Pori rivalis Nutt.

Pennsylvania cinquefoil

Platte cinquefoil

beauty cinquefoil

brook cinquefoil

PRIM Primula primrose

PROB Proboscidea - Martynia devilsclaws

Prvu Prunella vulgarts L. selfheal

common selfheal

PSIL Psilostrophe

PsCo

Pssp

Psta

PSOR

Psla

Este

Ptaq Pteridium aquilinum (L.) Kuhn

Ptaqp pubescens Underw.

PTER Pterospora

Ptan-2 andromedea Iutt. paperflower

whitestem paperflower

greenstem paperflower

woolly paperflower

scurfpea

breadroot scurfpea beaverbread scurfpea

lemon scurfpea

slender scurfpea slimflower scurfpea

bracken

western bracken

Ptiloria See Stephanomeria

pinedrops

woodland pinedrops 

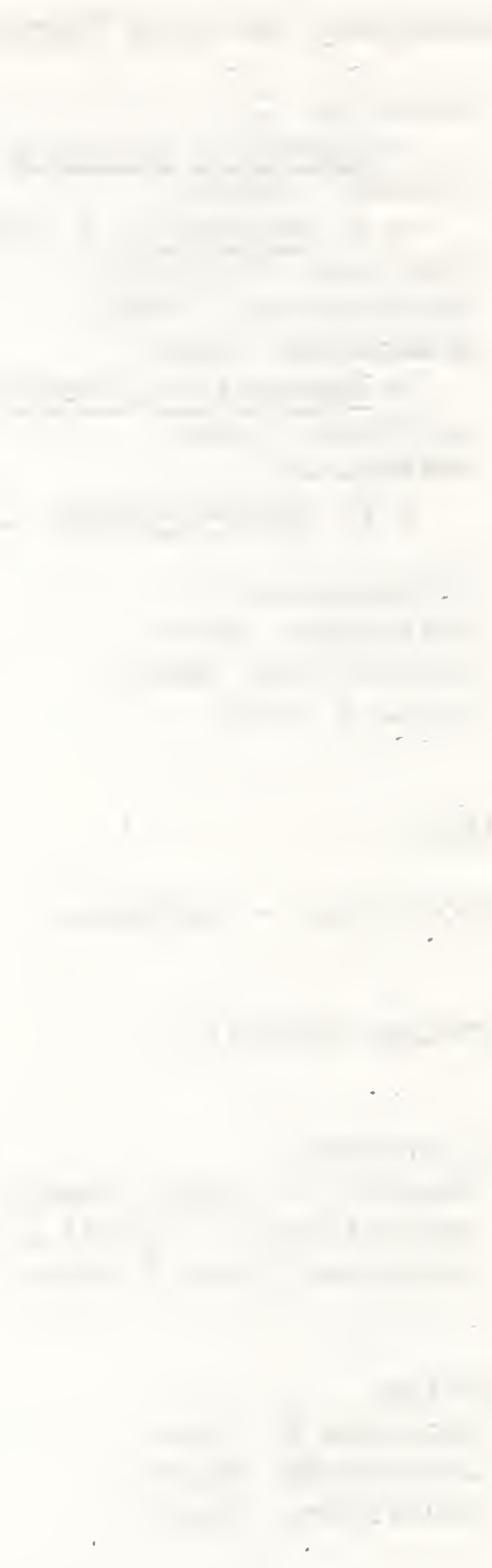
PYRO Pyrola

Pyel elliptica Nutt.

Pypi picta J. E. Smith secunda See Ramischia secunda pyrola

waxflower pyrola

whitevein pyrola.

Pymu Pyrrhopappus multicaulis DC.

false-dandelion

Quamocildion multiflorum See Mirabilis multiflora

Guamoclit See Ipomoea

Rase

Ramischia secunda (L.) Garcke

= Pyrola secunda $\mathrm{L}$.

sidebells-pyrola

sidebells pyrola

RANU Ranunculus

buttercup

(crowfoot)

buttercup

Raaq

Racy

Rafl

Rahy

Rama

aquatilis L. I/

= Batrachium aquatile (L.) Wimm.

aquatic buttercup

watercrowfoot buttercup cymbalaria Pursh flammula L. hydrocharoides A. Gray macranthus Scheele

desert buttercup shore buttercup creeping buttercup spearwort buttercup small-leaf aquatic buttercup showy buttercup

RATI Ratibida

prairie-coneflower

RHIN

Rhinanthus

rigidus Chab.

yellowrattle

yellowrattle

rattleweed

Rhri

RHYN Rhynchosia $=\underline{\text { Dolicholus }}$

rosarybean

rhynchosia

Ricinus see "Shrubs"

RIVI

Rivina

humilis $L$.

rougeplant

bloodberry

bloodberry rouge-

plant

I/ Typical $R$. aquatilis is an Old World species; Ranunculus aquatilis var. capillaceus (Thuill.) DC. is the name for plants of our area; . trichophylius Chaix is a symonym of this. 

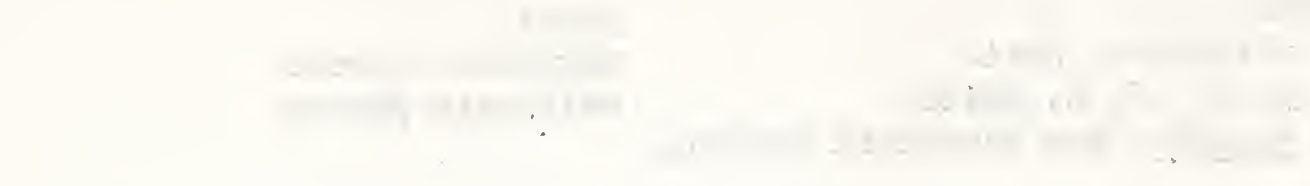

$=0$ 
Forbs List (continued)

RORI Rorippa rorippa

Rona nasturtium-aquaticum (L.) Schinz \& Thell. watercress

Roob obtusa (Nutt.) Britt. fieldcress

Rosy sylvestris (L.) Besser yellowcress

obtuse fieldcress

yellow fieldcress
RUDB Rudbeckia

Ruhi hirta I.

Rula laciniata I.

Ruoc occidentalis Nutt.

RUNE Rumex

Ruac acetosella I.

Ruco conglomeratus Murr.

Rucr

Ruhy

Ruob crispus $L$. hymenosepalus Torr. obtusifolius L.
SAGI-2 Sagina

SAGI-I Sagittaria

Saka Salsola kali L. $=\underline{\text { S. pestifer }}$ A. Nels.

SALV Salvia See also "Shrubs"

Saar

Saco

Sare

SAMO Samolus

SANG Sanguisorba

SAPO

Seof

Sava

SART Sartwellia columbariae Benth. reflexa Hornem. coneflower

black-eyed-susan

cutleaf coneflower

niggerhead

dock (sorrel)

dock

sheep sorrel.

clustered dock

curly dock

canaigre (wild-rhubarb)

bitter dock

pearlwort

arrowhead

Russianthistle

sage

Arizona sage

chia sage

RockyMountain sage

California chia

waterpimpernel I]

burnet

soapwort

bouncing-bet

cow soapwort

gypsumweed

If There are several "water-pimernels", incl. Veronica anagallis-aquatica. 

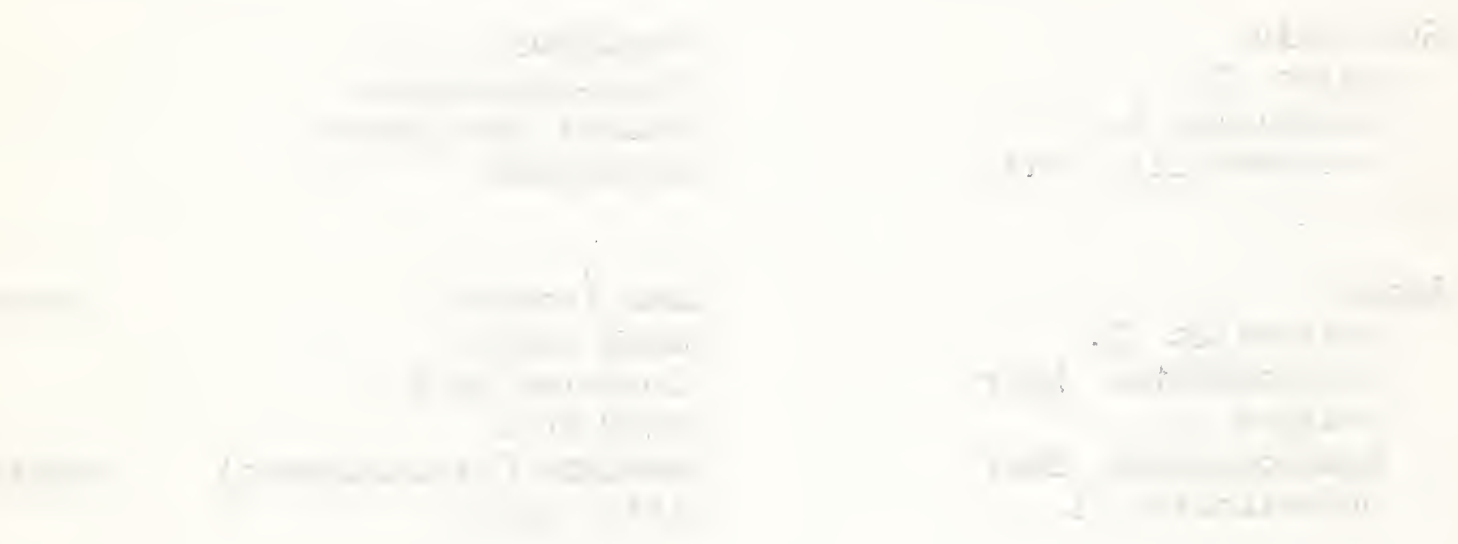

$\checkmark$

1.7 1 1

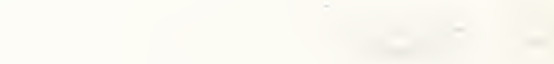

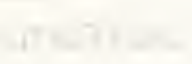
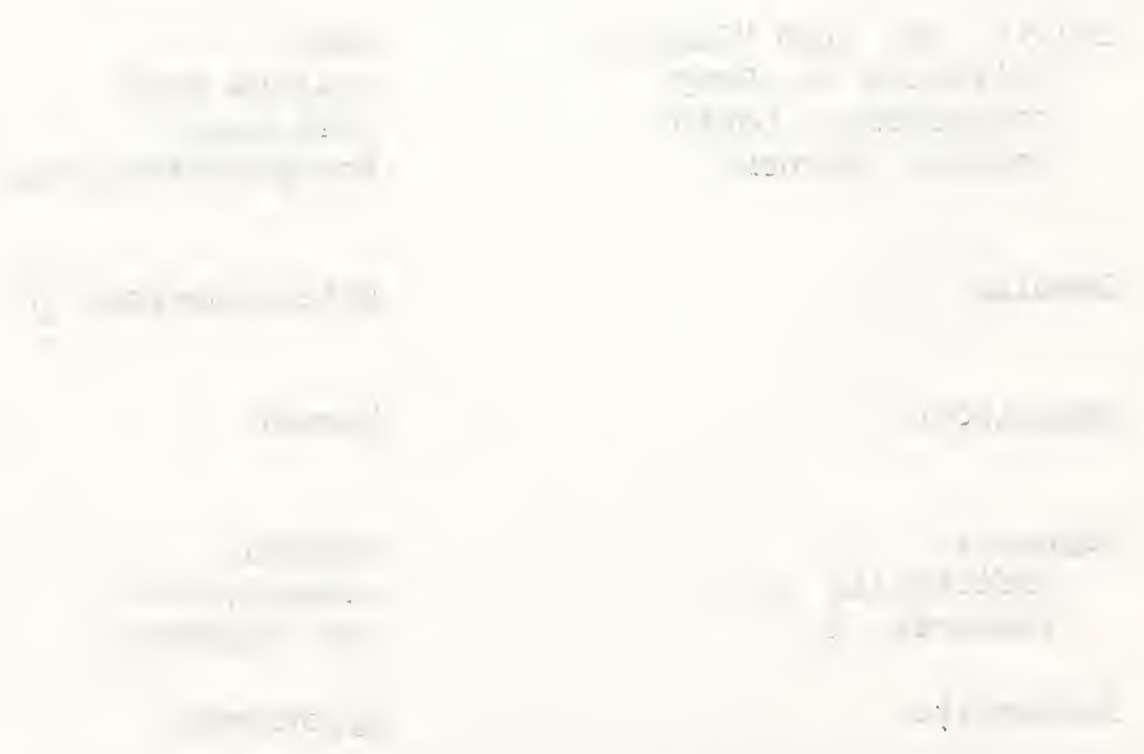

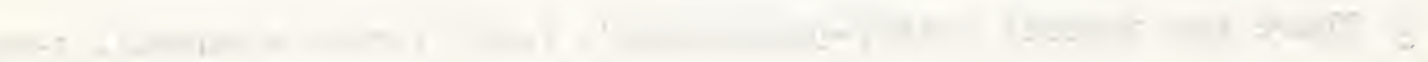




\section{Forbs List (continued)}

SAXI Saxifraga

SCRO Scrophularia

SCUT Scutellaria

Scla lateriflora L. figwort

skull cap

sideflowering skullcap
Caucasian skullcap

$\begin{array}{lc}\text { SEDU } & \begin{array}{c}\text { Sedum } \\ \text { Sest } \\ \text { stenopetalum Pursh }\end{array} \\ \text { SENE } & \text { Senecio } \\ \text { Sefe } & \text { fendleri A. Gray } \\ \text { Selo } & \text { longilobus Benth. } \\ \text { Semu } & \text { multilobatus Torr. \& Gray } \\ \text { Sene } & \text { neomexicanus A. Gray } \\ \text { Sesa } & \text { salignus DC. } \\ \text { Sesp } & \text { spartioides Torr. \& Gray } \\ \text { Seul } & \text { uintahensis A. Nels } \\ \text { Sevu } & \text { vulgaris L. }\end{array}$

Sihe

Sisp

Sine spinosa I.
SIDA Sida

sidalcea

New-Mexican checkermallow

stonecrop

wormleaf stonecrop

groundsel

notchedleaf groundsel Fendler groundsel threadleaf groundsel lobeleaf groundsel New-Mexican groundsel willow groundsel broom groundsel Uinta groundsel common groundsel

sida

alkali sida prickly sida sidalcea

SIIE Silene

Siac

Sian

Sila

Sisc

Sial Sisymbrium altissimum $I$.

SISY Sisyrinchium demissum Greene

acaulis $I$. antirrhina L. laciniata Cav. scouleri Hook. silene

moss silene sleepy silene Mexican silene
SIUM Sium

Sisu suave Walt.

$$
\begin{aligned}
& \text { silene } \\
& \text { (catchfly, campion*) } 1 / \\
& \text { moss carnion } \\
& \text { sleepy catchfiy } \\
& \text { Mexican campion } \\
& \text { Scouler silene }
\end{aligned}
$$

tumblemustard

blue-eyed-grass

blue-eyed-grass

waterparsnip

waterparsnip

1 Campion is generally accepted name for Lychnis. 

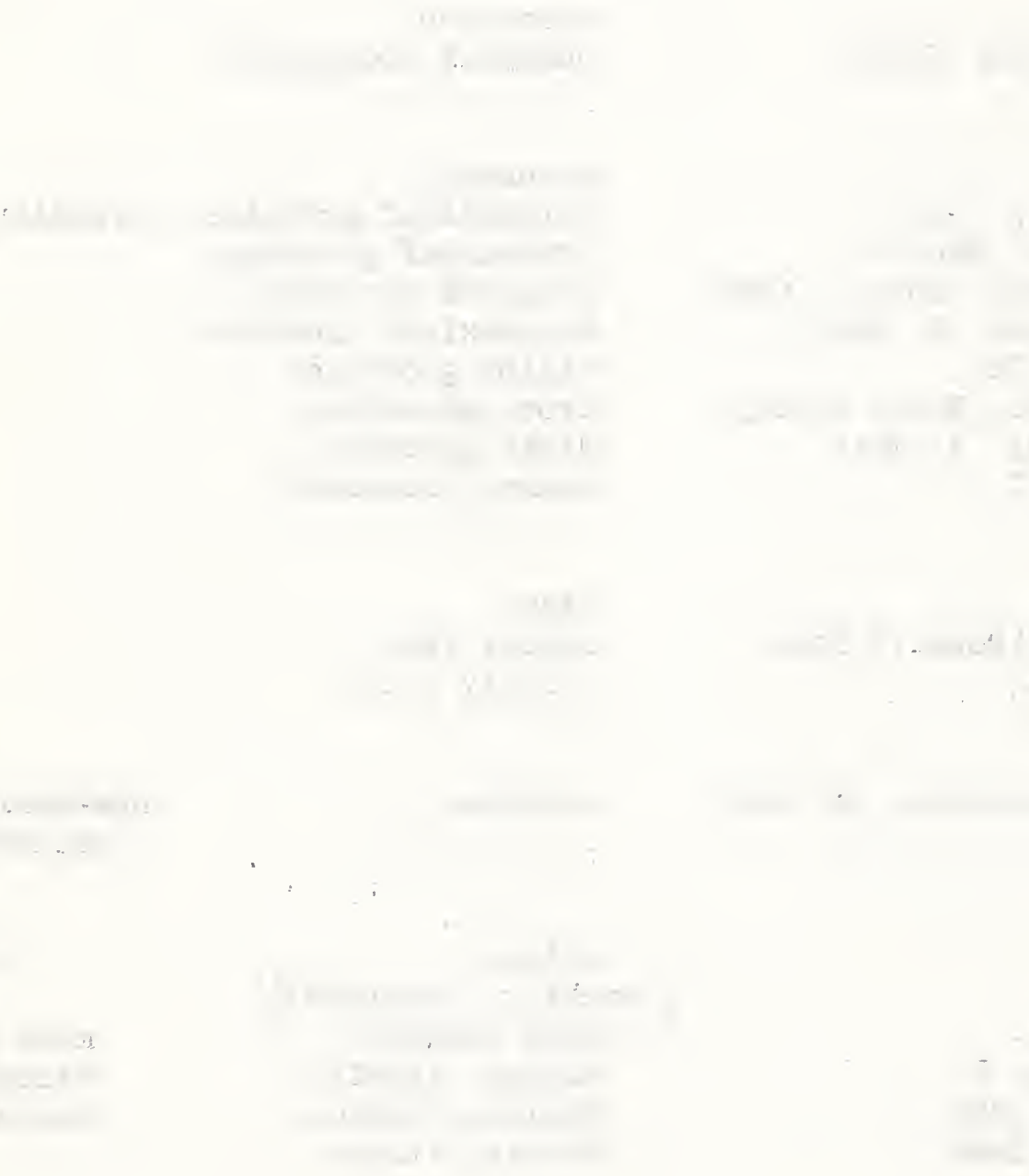
Forbs List (continued)

\begin{tabular}{|c|c|c|c|}
\hline SiIIL & Smilacina $=$ Vagnera & smilac & $\begin{array}{c}\text { Solomonplume } \\
\text { (false-Solomonseal) }\end{array}$ \\
\hline Smra & racemosa (I.) Desf. & falsemSolomonseal & $\begin{array}{l}\text { feather } \\
\text { Solomonplume }\end{array}$ \\
\hline Smst & stellata (I.) Desf. & starry smilac & $\begin{array}{l}\text { starry } \\
\text { Solomonplume }\end{array}$ \\
\hline SOLA & Solanum & nightshade & \\
\hline Soel & elaeagnifolium Cav. & $\begin{array}{l}\text { white horsenettle } \\
\text { (bullnettle) }\end{array}$ & $\begin{array}{l}\text { silverleaf } \\
\text { night shade }\end{array}$ \\
\hline $\begin{array}{l}\text { Sofe } \\
\text { Sohe }\end{array}$ & $\begin{array}{l}\text { fendleri A. Gray } \\
\text { heterodoxum Dunal }\end{array}$ & $\begin{array}{l}\text { Fendle } \mathrm{r} \text { potato } \\
\text { melonleaf nightshade }\end{array}$ & \\
\hline Soja & jamesii Torr. & $\begin{array}{l}\text { wild potato } \\
\text { a true wild potato }\end{array}$ & -..- James potato \% \\
\hline Soni & $\begin{aligned} & \text { nigrum I. } \\
& \text { NOTE: Nany plants referre } \\
& \text { prove to be Solanu }\end{aligned}$ & $\begin{array}{l}\text { black night shade } \\
\text { ed to Solanum nigrum } \\
\text { num nodiflorum Jacq. }\end{array}$ & \\
\hline Soro & rostratum Dunal & buffalombur & $\begin{array}{l}\text { buffalo-bur } \\
\text { nightshade }\end{array}$ \\
\hline $\begin{array}{l}\text { Sotr } \\
\text { Sosera }\end{array}$ & $\begin{array}{l}\text { triflorum Nutt. } \\
\text { xantii A. Gray }\end{array}$ & $\begin{array}{l}\text { cutleaf night shade } \\
\text { purple night shade }\end{array}$ & \\
\hline SOLI & Solidago & goldenrod & \\
\hline Soal & altissima L. & tall goldenrod & \\
\hline Soca & canadensis $\mathrm{L}$ & Canada goldenrod & \\
\hline Sode & decumbens $G_{\text {reene }}$ & decumbent goldenrod & \\
\hline Somi & missouriensis Nutt. & i'issouri goldenrod & \\
\hline $\begin{array}{l}\text { Sona } \\
\text { Sooc }\end{array}$ & $\begin{array}{l}\text { nana Nutt. } \\
\text { occidentalis (Nutt.) }\end{array}$ & baby goldenrod & \\
\hline \multirow[t]{2}{*}{ Sope } & $\begin{array}{l}\text { Torr. \& Gray } \\
\text { petradoria Blake }\end{array}$ & $\begin{array}{l}\text { western goldenrod } \\
\text { rock goldenrod }\end{array}$ & \\
\hline & $\begin{array}{l}\text { Sophia See Descurainia } \\
\text { Sophora See "Shrubs" }\end{array}$ & & \\
\hline SPAR -2 & Sparganium & burreed & \\
\hline $\mathrm{SPEC}$ & Specularia = Triodanis & Venus-lookingglass & \\
\hline Spbi & $\begin{array}{c}\text { biflora (Ruiz \& Pavon) } \\
\text { Fisch. \& Mley. }\end{array}$ & small lookingglass & \\
\hline Sppe-2 & $\begin{array}{l}=\text { Triodanis biflora } \\
\text { perfol (Ruiz \& } \\
=\text { Triodanis perfoliata }\left(L_{0}\right)\end{array}$ & $\begin{array}{l}\text { \& Pavon) Greene } \\
\text { clasping } \mathrm{V}_{\mathrm{e}} \text { nus-iookin } \\
\text { Nieuwl. }\end{array}$ & aggla ss \\
\hline
\end{tabular}




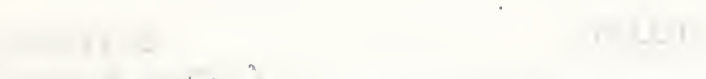

$1-\cdots+13$

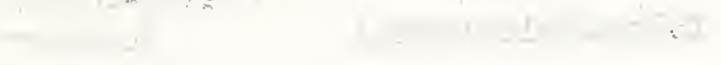

$-1+1+20$

$-1 \cdot$

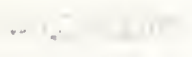

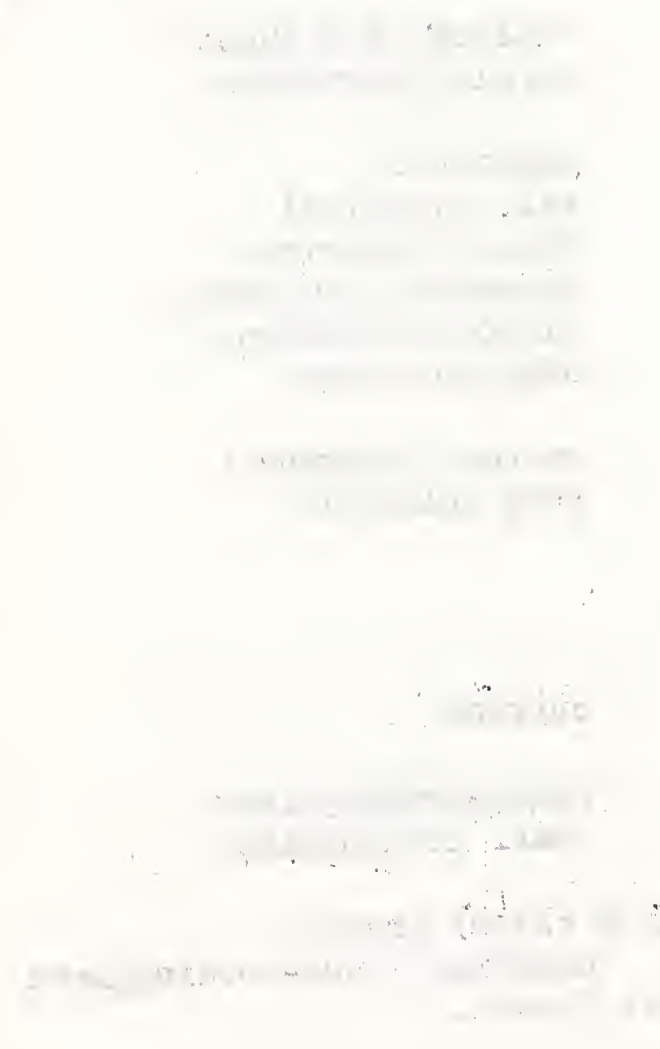

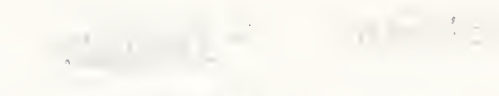

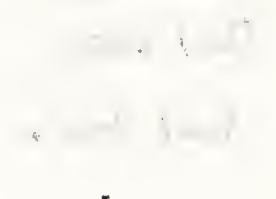

$\rightarrow$

:
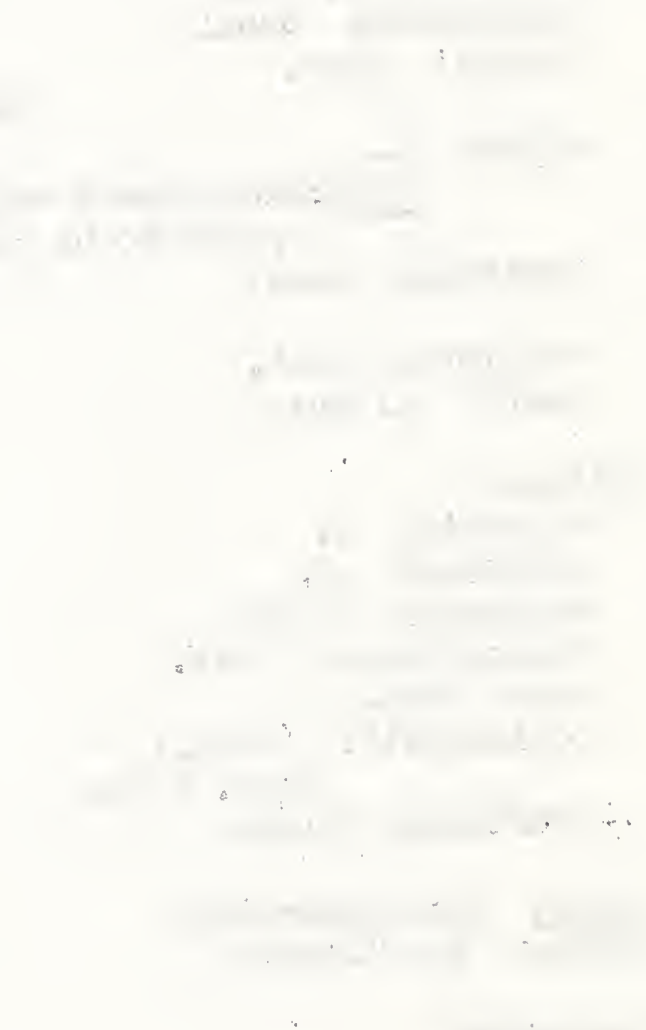

:

,

. $\quad . \quad 4$

$=-1$ 
Forbs List (continued)

\begin{tabular}{|c|c|c|c|}
\hline SPHA & Sphaeralcea & $\begin{array}{l}\text { globemallow } \\
\text { (hollyhock) }\end{array}$ & globemallow \\
\hline Spam & ambigua $A_{0} G_{r a y}$ & desertmallow & $\begin{array}{l}\text { desert \& } \\
\text { globemallow }\end{array}$ \\
\hline Spfe & $\begin{array}{l}\text { fendleri A. Gray } \\
\text { grandiflora See Iliamna grand }\end{array}$ & $\begin{array}{l}\text { Fendler globemallow } \\
\text { flora }\end{array}$ & $\rightarrow \infty-\infty$ \\
\hline SPIR & Spiranthes & ladies-tresses & \\
\hline STAN & $\begin{array}{l}\text { Stanleya } \\
\text { Steironema ciliatum See Lysim }\end{array}$ & $\begin{array}{l}\text { desertplume } \\
\text { (princesplume) } \\
\text { ichia ciliata }\end{array}$ & princesplume \\
\hline $\begin{array}{l}\text { STEL } \\
\text { Stja } \\
\text { Stlom2 } \\
\text { Stme }\end{array}$ & $\begin{array}{l}\text { Stellaria } \\
\text { jamesiana Torr. } \\
\text { longipes Goldie } \\
\text { media (I.) Cyrill. }\end{array}$ & $\begin{array}{l}\text { starwort } \\
\text { (chickweed) } \\
\text { tuber starwort } \\
\text { longstalk starwort } \\
\text { chickweed }\end{array}$ & starwort \\
\hline STEP & Stephanomeria $=$ Ptiloria & wirelettuce & \\
\hline $\begin{array}{l}\text { STRE } \\
\text { Stam }\end{array}$ & $\begin{array}{l}\text { Streptopus } \\
\text { amplexifolius (L.) DC. }\end{array}$ & $\begin{array}{l}\text { twistedstalk } \\
\text { claspleaf twistedstalk }\end{array}$ & \\
\hline SUAE & Suaeda = Dondia & seepweed & \\
\hline SWER & Swertia = Frasera & $\begin{array}{l}\text { swertia } \\
\text { eengentian) }\end{array}$ & swertia \\
\hline Sipe & $\begin{array}{l}\text { perennis I. } \\
\quad=\mathrm{S} \text {. scopulina Greene }\end{array}$ & alpine-bog swertia & \\
\hline Swra & $\begin{array}{l}\text { radiata (Kellogg) Kuntze } \\
\text { = Frasera speciosa Griseb. } \\
\text { recurva See Halenia recurva }\end{array}$ & deersmears swertia & showy swertia \\
\hline Srot & $\begin{array}{l}\text { utahensis (Jones) St. John } \\
=\text { Frasera paniculata Torr. }\end{array}$ & elkweed swertia & $-\infty-\infty \infty$ \\
\hline $\begin{array}{l}\text { TAGE } \\
\text { Tale }\end{array}$ & $\begin{array}{l}\text { Tagetes } \\
\text { Iemmonii A, Cray }\end{array}$ & marigold & $-m-m-\infty$ \\
\hline Tami & micrantha Cav. & wild marigold & $\begin{array}{l}\text { little mari- } \\
\text { gold }\end{array}$ \\
\hline TARA & $\begin{array}{l}\text { Taraxacum } \\
\text { Taraxacum } \Rightarrow \text { Leontodon, in part }\end{array}$ & dandelion & \\
\hline THAL & Thalictrum & meadowrue & \\
\hline
\end{tabular}


THET Thelesperma

Thlo longipes A. Gray

Thme

Thsu megapotamicum (Spreng.) Kuntze

THER Thermopsis

Thmo-1 montana Nutt.

Thpi greenthread

cota

Hopi-tea

Navajo-tea cota greenthread

Hopi-tea greenthread

Navajo-tea

greenthread

thermopsis goldenpea

(big yellowpea)

mountain goldenpea

pine goldenpea.

NOTE: Thermopsis pinetorum is regarded by some botanists as a synonym of $\mathrm{T}$. divaricarpa A. Nels.

THLA Thlaspi

Thfe fendleri A. Gray

pennycress

candytuft

NOrI: Candytuft is the cominonly accepted name for Iberis;

wildcandytuft or Fendler

pennycrest is suggested for a corinon name.

THYS Thysanocarpus

TIDE Tidestromia

Tila

Tiob

TILI Tier

\section{Tillaea}

erecta Hook, \& Arn. lacepod

tidestromia.

annual tidestromia

shrubby tidestromia

NOLE: Sinrubby tidestromia would be more descriptive of Tidestromia suffruticosa.

pigmyweed pigmyweed woolly

tidestromia

honeysweet

tidestromia pygmyweed sand

pygmyweed \%

Tithymalus See Nuphorbia

TRAD Tradescantia

spiderwort

TRAG-2 Tragia

noseburn

TRAG-I Tragopogon

Trpo porrifolius L.

goatsbeard

salsify

salsify

Trpis-2 pratensis $L$.

meador goatsbeard meadow salsify

NOTE: Goatsbeard is accepted cormon name for Aruncus.

\% Suggested comrion names. 
$+$

r

$$
\text { ... }
$$

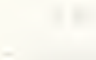

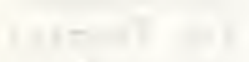

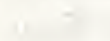

$-$
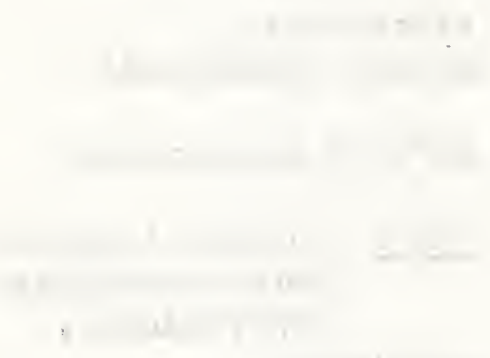

t) n the 
Forbs List (continued)

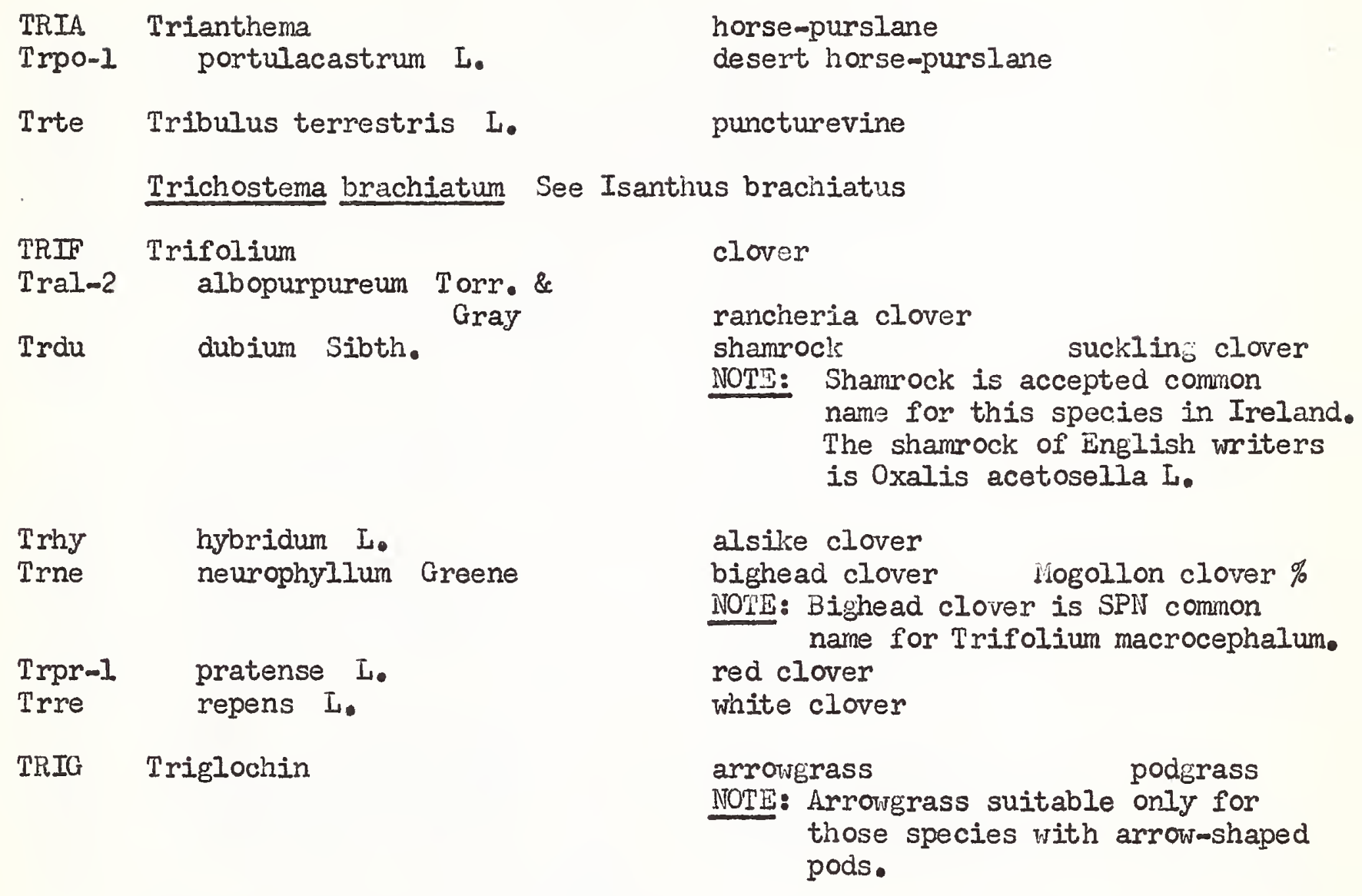

Triodanis See Specularia

INOFE: Triodanis, restored by $\mathrm{Dr}$. IICVaugh, is not regarded as a good genus by many botanists.

URTI Urtica

nettle

Vagnera See Smilacina

VALE Valeriana

VERA Veratrum

Veca californicum Durand

VERB-1 Verbascum

Veth thapsus I.

Vevi virgatum Stokes tobaccoroot;

valerian

false-hellebore

false-hellebore

California

falsedheliebore

mullein

flaninel mulle in

purple-stamen mullein

$\%$ - Suggested comon name 
$\therefore \because \quad \quad \therefore \quad \quad \quad \quad \cdots$

, $\because \because$
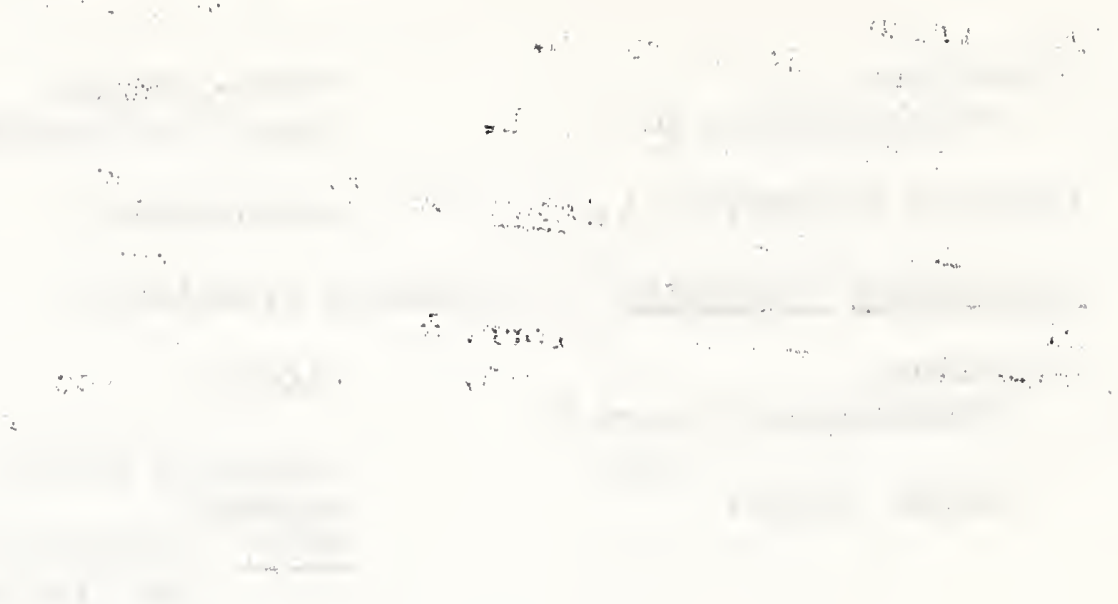

$x^{2}+x^{2}$

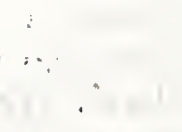

i

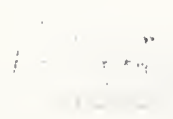


Forbs Iist (continued)

\begin{tabular}{|c|c|c|c|}
\hline $\begin{array}{l}\text { VeRB-2 } \\
\text { Vebi } \\
\text { Vebr } \\
\text { Veha } \\
\text { Vewr }\end{array}$ & $\begin{array}{l}\text { Verbena } \\
\text { bipinnatifida irutt. } \\
\text { bracteata Lag. \& Rodr. } \\
\text { hastata L. } \\
\text { wrightii A. Gray }\end{array}$ & $\begin{array}{l}\text { verbena } \\
\text { Dakota verbena } \\
\text { bigbract verbena } \\
\text { blue verbena } \\
\text { Wrights verbena }\end{array}$ & \\
\hline $\begin{array}{l}\text { VERB-3 } \\
\text { Veen }\end{array}$ & $\begin{array}{l}\text { Verbesina } \\
\text { encelioides (Cav•) Benth. } \\
\text { \& Hook. }\end{array}$ & $\begin{array}{l}\text { crownbeard } \\
\text { golden crownbeard }\end{array}$ & \\
\hline $\begin{array}{l}\text { VERO } \\
\text { Veam }\end{array}$ & $\begin{array}{l}\text { Veronica } \\
\quad \text { americana }\left(R_{a} f_{\bullet}\right) \text { Schwein. }\end{array}$ & $\begin{array}{l}\text { speedwell } \\
\text { American brooklime }\end{array}$ & $\begin{array}{l}\text { American } \\
\text { speedwell }\end{array}$ \\
\hline Vean & anagallis-aquatica $\mathrm{L}$. & water speedivell & \\
\hline $\begin{array}{l}\text { VICI } \\
\text { Viam } \\
\text { Viex } \\
\text { Vile } \\
\text { Vipu-I } \\
\text { Visa }\end{array}$ & $\begin{array}{l}\text { Vicia } \\
\text { americana iruhl. } \\
\text { exigua IJutt. } \\
\text { leucophaea Greene } \\
\text { pulchella H.B.K. } \\
\text { sativa L. }\end{array}$ & $\begin{array}{l}\text { vetch } \\
\text { American vetch } \\
\text { slim vetch } \\
\text { Hogollon vetch } \\
\text { sweetclover vetch } \\
\text { fodder vetch }\end{array}$ & comnon vetch \\
\hline $\begin{array}{l}\text { VIGU } \\
\text { Vian } \\
\text { Vico } \\
\text { Vide } \\
\text { Videp }\end{array}$ & $\begin{array}{r}\text { Viguiera }=\text { Gymmolomia } \\
\text { annua (Jones) Blake } \\
\text { cordifolia A. Gray } \\
\text { deltoidea A. Gray } \\
\text { parishii (Greene) Vasey } \\
\text { \&orese } \\
\text { This variety only } \\
\text { goldeneye species } \\
\text { arborescent), delt } \\
\text { suggested as a mor } \\
\text { shrubby goldeneye }\end{array}$ & $\begin{array}{l}\text { goldeneye } \\
\text { annual goldeneye } \\
\text { heartleaf goldeneye } \\
\text { shrubby goldeneye } \\
\text { n }\end{array}$ & $\begin{array}{l}\text { Parish golden- } \\
\text { eye }\end{array}$ \\
\hline $\begin{array}{l}\text { Vimu } \\
\text { Viov }\end{array}$ & $\begin{array}{l}\text { longifolia (Robins. \& Greenm•) } \\
\text { Blak } \\
\text { multiflora (Nutt.) Blake } \\
\text { ovalis Blake }\end{array}$ & $\begin{array}{l}\text { showy goldeneye } \\
\text { ovalleaf goldeneye }\end{array}$ & \\
\hline
\end{tabular}

Vincetoxicum productum See Gonolobus productus

VIOL Viola

Viad

Vica

Vine

Vinu

Vipe

Vipu-2 adunca J. . S Smith canadensis $L_{\text {. }}$ nephrophylla Greene nuttallii Pursh pedatifida G. Don purpurea
. Kellogg violet

hook violet

Canaca violet wanderer violet Nuttall violet larkspur violet goosefoot violet prairie violet 


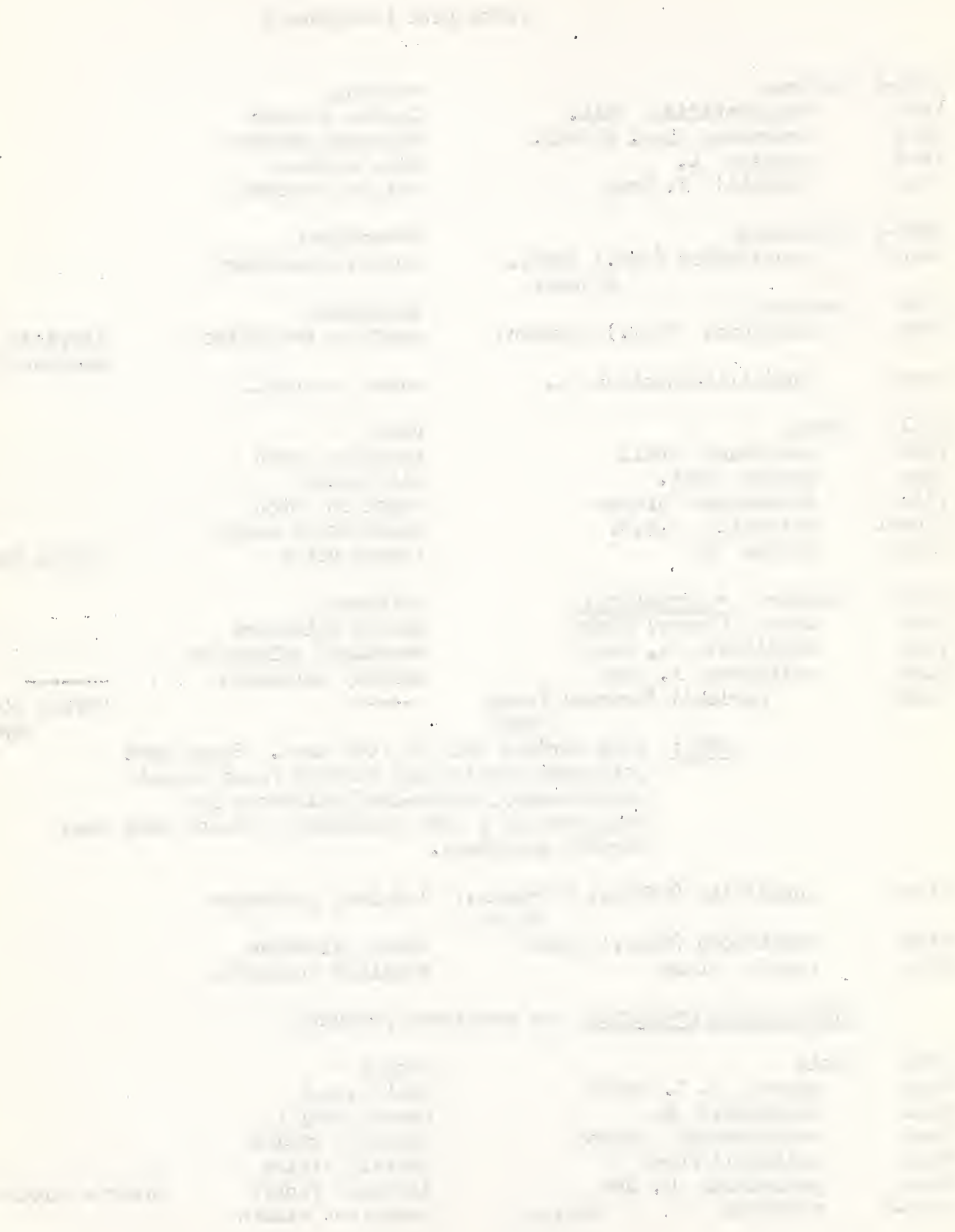




\section{- Forbs List (continued)}

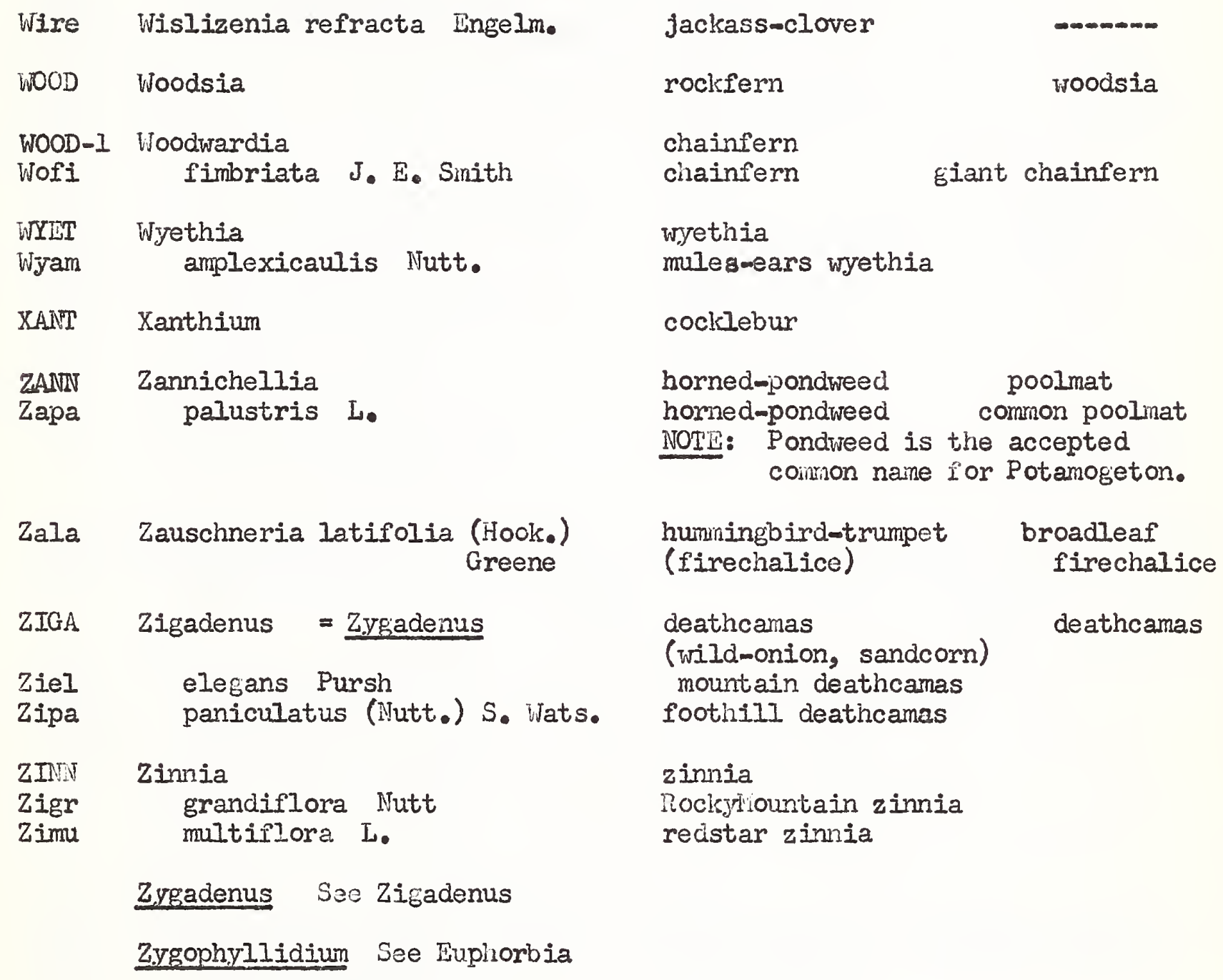




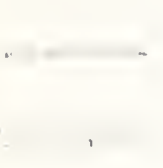

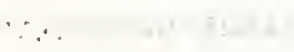

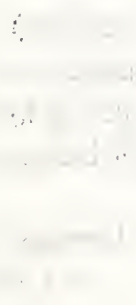

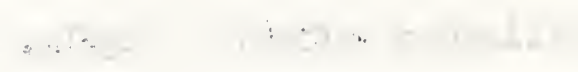

- $\quad 2=0$
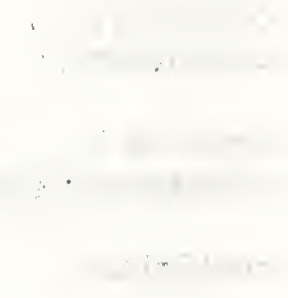

$+\ldots$

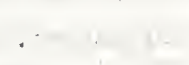

$\forall$

, $\quad$,

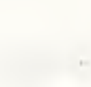

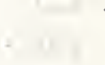

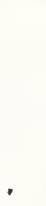

.

(b) 


\begin{tabular}{|c|c|c|}
\hline $\begin{array}{l}\text { ABIE } \\
\text { Abco } \\
\text { Abla } \\
\text { Ablaa }\end{array}$ & $\begin{array}{l}\text { Abies } \\
\text { concolor (Gord. \& Glend.) Lindl. } \\
\text { lasiocarpa (Hook.) Nutt. } \\
\text { arizonica (Merriam) Lemm. }\end{array}$ & $\begin{array}{l}\text { fir } \\
\text { white fir } \\
\text { subalpine fir } \\
\text { corkbark fir }\end{array}$ \\
\hline ABUT & Abutilon & Indianmallow \\
\hline $\begin{array}{l}\text { ACAC } \\
\text { Acan } \\
\text { Acco-1 }\end{array}$ & $\begin{array}{l}\text { Acacia } \\
\text { angustissima (Mill.) Kuntze } \\
\text { constricta Benth. }\end{array}$ & $\begin{array}{l}\text { acacia } \\
\text { whiteball acacia } \\
\text { mescat acacia } \\
\text { (whitethorn) }\end{array}$ \\
\hline $\begin{array}{l}\text { Acfa } \\
\text { Acgr }\end{array}$ & $\begin{array}{l}\text { famesiana (L.) Willd. } \\
\text { greggii A. Gray }\end{array}$ & $\begin{array}{l}\text { sweet acacia } \\
\text { catclaw acacia }\end{array}$ \\
\hline
\end{tabular}

Acli Acalypha lindheimeri Muell. -Arg. crimson acalypha abutilion

prairie acacia mescat acacia

Lindheimer copperleaf

ACAM Acamptopappus goldenhead

Acsp sphaerocephalus (Harv. \& Gray) A. Gray rayless goldenhead

ACER Acer

Acgl glabrum Torr.

Acgr-1 grandidentatum Nutt.

Acne negundo $L$.

Adelia See Forestiera. maple

Rocky Mountain maple

bigtooth maple

boxelder

\begin{abstract}
AGAV Agave
Agat atrovirens Karw.

Agde

Agka

Agpa-2

Agpa-3 parryi Engelm.

Agpa-4 parviflora Torr.

Agsc-3 schottii Engelm.

Agut utahensis Engelm.
\end{abstract}

ATINU

Alob

Alte
Alnus

oblongifolia Torr. tenuifolia Nutt. agave

pulque agave -- a Mexican species

desert agave

Kaibeb agave

Palmer agave

(centuryplant)

Parry agave

(mescal)

small-flowered agave

Schott agave

(amole)

Utah agave

alder

Arizona alder

thinleaf alder

(mountain alder) 

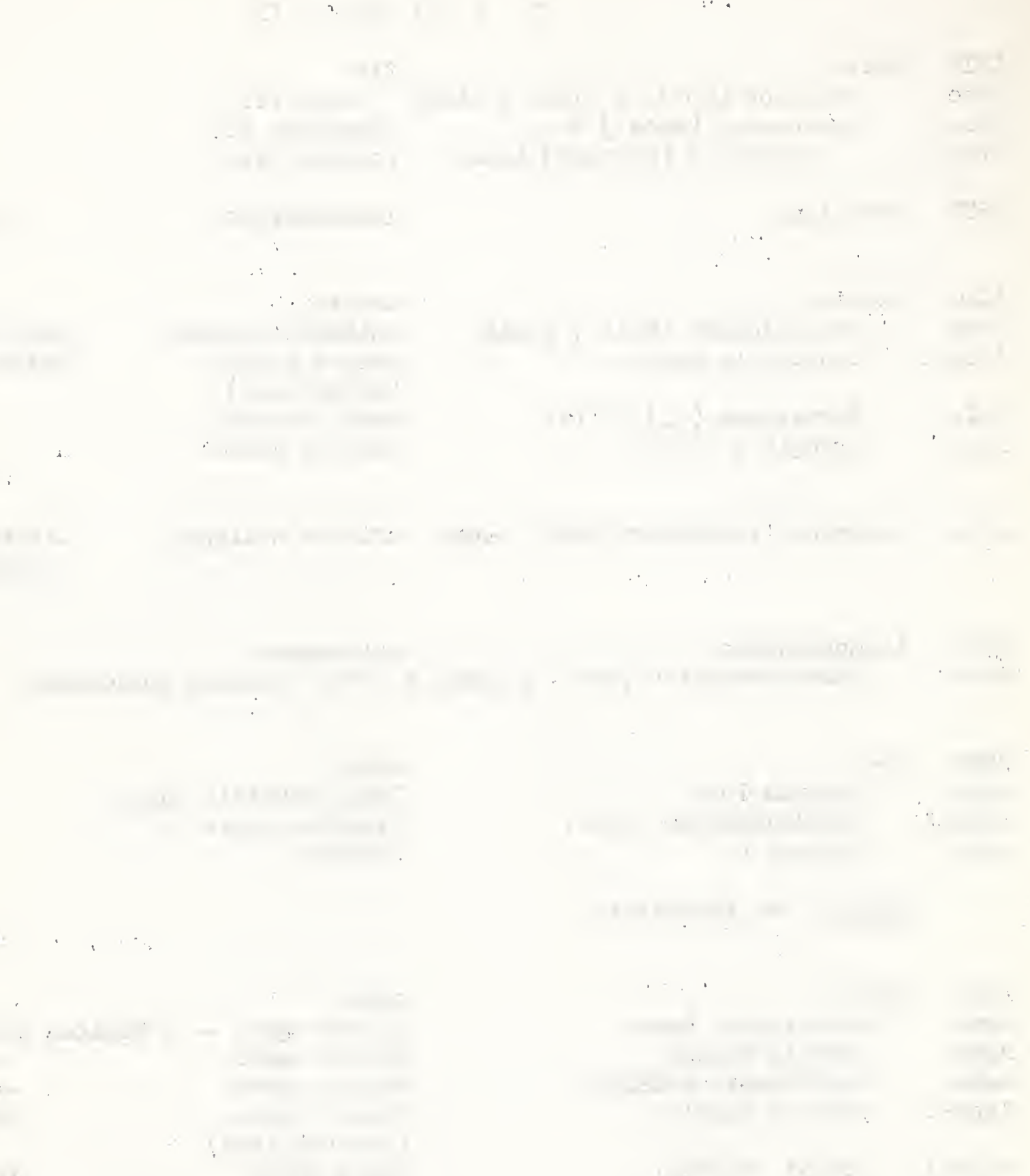
Alwr Aloysia wrightil (A.Gray) Heller Wrights lippia Wrights lemon-verbena\% = Iippia wrightii A. Gray

AMEL

Ammo

Amor

Ampo

Amut

AMOR Amorpha

Amca californica Nutt.

Amfr-1 fruticosa L.

Amfr-2 Amphipappus fremontii Torr. \& Gray

ANIS

Anth

Anisacanthus

thurberi (Torr.) A. Gray serviceberry

Mormon serviceberry

mountain serviceberry

cluster serviceberry

Utah serviceberry

amorpha

stinkingwillow amorpha.

indigobush amorpha

\section{California}

amorpha

chaff'bush

(broomweed)

anisacanth

desert-honeysuckle Thurber anisacanth

NOTE: There are some true honeysuckles (Lonicera

spp.) growing in the southwestern U.S. and northern Mexico deserts. Desert-honeysuckie not a very distinctive name. See also Beloperone.

APLO Aplopappus (also spelled Haplopappus) See Forbs List.

$\%$ Suggested common name.

1/ Serviceberry species hard to tell apart. G. N. Jones, "American Species of Amelanchier" (Univ. Ill. Biol. Monog. 20: 1 - 126. 1946) regards A. mormonica and A. oreophila as synonyms of A. utahensis, and A. polycarpa as a synonum of A. pumila Nutt. The Forest Service Check List reduces A. oreophila to a synonum of A. utahensis. Kearney and Peebles, Arizona Flora, 1951, treat the species as given above. 


$$
\text { N. }
$$
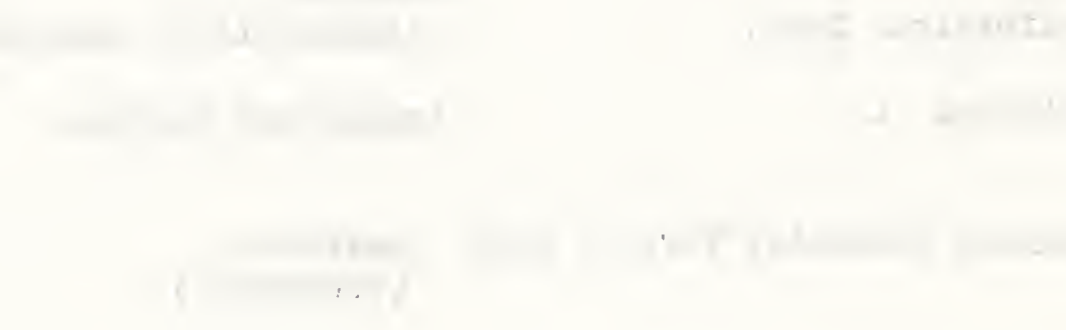

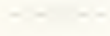
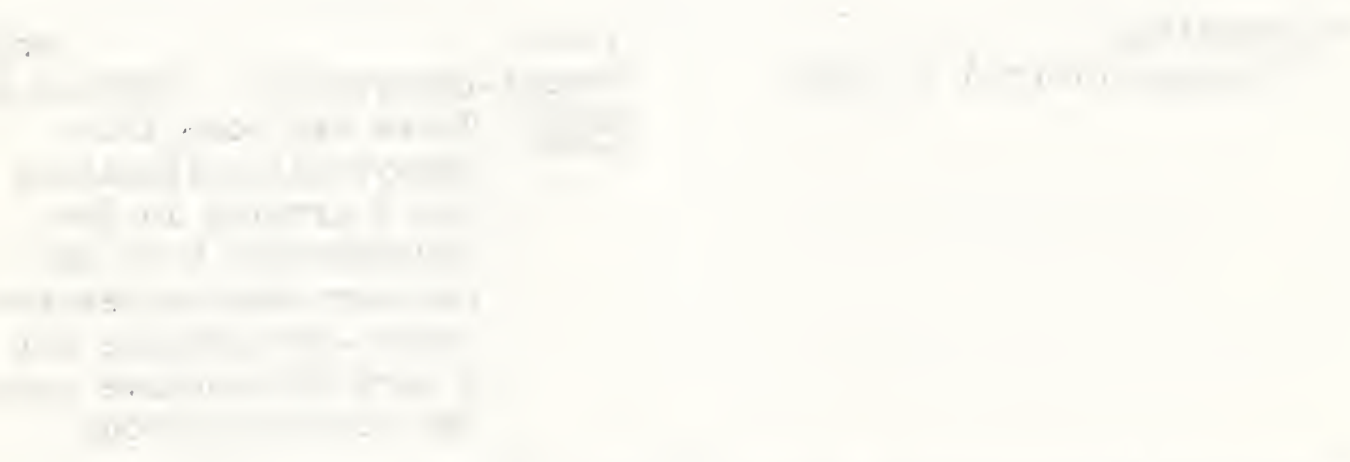
- Trees and Shrubs List (continued)

$\begin{array}{lc}\text { ARBU } & \begin{array}{c}\text { Arbutus } \\ \text { Arar-2 } \\ \text { arizonica (A. Gray) Sarg } \\ \text { texana Buckl. }\end{array} \\ \text { ARCE } & \text { Arceuthobium = Razoumowsky } \\ & \\ \text { ARCT-1 } & \text { Arctostaphylos } \\ \text { Arpa-3 } & \text { patula Greene } \\ \text { Arpr } & \text { pringlei Parry } \\ \text { Arpu-2 } & \text { pungens H.B.K. } \\ \text { Aruv } & \text { uva-ursi (L.) Spreng. } \\ & \\ \text { ARTE } & \text { Artemisia } \\ \text { Aran } & \text { annua L. } \\ \text { Arca-3 } & \text { cana Pursh } \\ \text { Ardr-2 } & \text { dracunculoides Pursh } \\ & \text { filifolia Torr. } \\ \text { Arfi } & \text { filone } \\ \text { Arfr-2 } & \text { franserioides Greene } \\ \text { Arfr-1 } & \text { frigida Willd. } \\ \text { Arlu } & \text { ludoviciana Nutt. } \\ \text { Armo } & \text { nova A. Nels. } \\ \text { Arpy } & \text { pygmaea A. Gray } \\ \text { Arsp } & \text { spinescens D. C. Eaton } \\ \text { Artr } & \text { tridentata Nutt. } \\ & \end{array}$

ATRI Atriplex

Atar argentea Nutt.

Atca canescens (Pursh) Nutt.

Atco confertifolia (Torr.\& Frem.) S. Wats. spiny saltbush

Athy hymenelytra (Torr.) S. Wats. desertholly

Atle lentiformis (Torr.) S. Wats. quailbrush

Atpo madrone

Arizona madrone

Texas madrone

dwarfmistletoe

manzanita

greenleaf manzanita

Pringle manzanita.

pointleaf manzanita

bearberry (kinnikinnick)

sagebrush

sweet wormwood

silver sagebrush

false-tarragon

sand sagebrush

Palse-tarragon sagebrush

ragweed sagebrush

estafiata

Louisiana wormwood

black sagebrush

pygny sagebrush

budsage

(buttonsage)

big sagebrush

fringed sagebrush

Louisiana sagebrush

bud sagebrush

saltbush (saltsage,

saltbush

sage*, spearscale,

redscale, arrowscale

wedgescale)

tumbling saltbush

NOTE: Tumbling orach is accepted conmon name for Atriplex rosea.

fourwing saltbush (chamiza) (estafietta)

fourwing saltbush

shadscale saltbush

desertholly saltbush

big saltbush (lenscale, quailbush)

desert saltbush

(cattle-spinach)

cattle saltbush

NOTE: Allscale saltbush is another suggested common name. 
Trees and Shrubs List (continued)

$\begin{array}{lc}\text { Atriplex (continued) } \\ \text { Atro } & \text { rosea L. } \\ \text { Atse } & \text { semibaccata R. Br. } \\ & \\ \text { AyEN } & \text { Ayenia } \\ \text { Aymi } & \text { microphylla A. Gray } \\ \text { Aypu } & \text { pusilla L. } \\ & \\ \text { BACC } & \text { Baccharis } \\ \text { Babr } & \text { brachyphylla A. Gray } \\ \text { Baem } & \text { emoryi A. Gray } \\ \text { Bagl } & \text { glutinosa Pers. } \\ \text { Bapt } & \text { pteronioides DC. } \\ \text { Basa-1 } & \text { sarothroides A. Gray A. Gray } \\ \text { Base } & \text { sergiloides A. Gray } \\ \text { Bavi } & \text { viminea DC. }\end{array}$

Beju Bebbia juncea (Benth.) Greene

Becie Beloperone californica Benth.

$B E R B$

Befe redscale

Australian saltbush

ayenia

littleleaf ayenia

dwarf ayenia

baccharis

shortleaf baccharis

Emory baccharis

seepwillow

yerba-de-pasmo

broom baccharis

squaw baccharis

mulefat baccharis

$$
\begin{gathered}
\text { seepwillow } \\
\text { baccharis } \\
\text { yerba-de-pasmo } \\
\text { baccharis }
\end{gathered}
$$

rush bebbia

California beloperone (chuparosa)

barberry

Colorado barberry

tocarpa

haematocarpa See Mahonia haematocarpa

repens See Mahonia repens

trifoliolata. See Mahonia trifoliolata

BETU Betula

Begl

fontinalis see B. occidentalis

glandulosa Michx.

occidentalis Hook.

= B. fontinalis Sarg.

Bogl Bouvardia glaberrima Engelm.

$$
=\underline{\text { B }} \text { ovata A. Gray }
$$

birch

bog birch

water birch

(red birch)

honeysuckle bouvardia
California beloperone

barberry water birch 


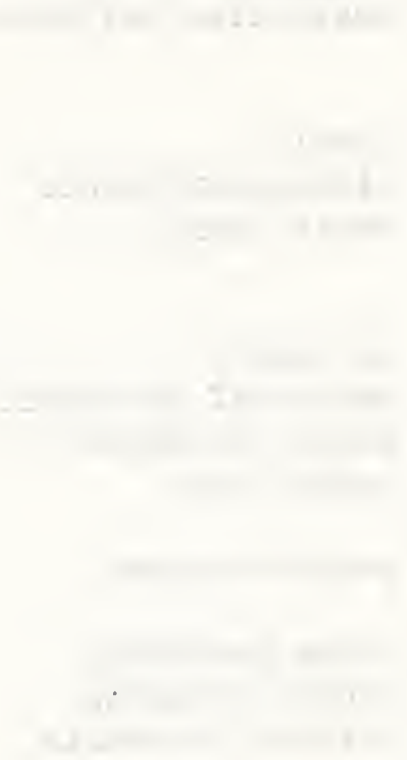




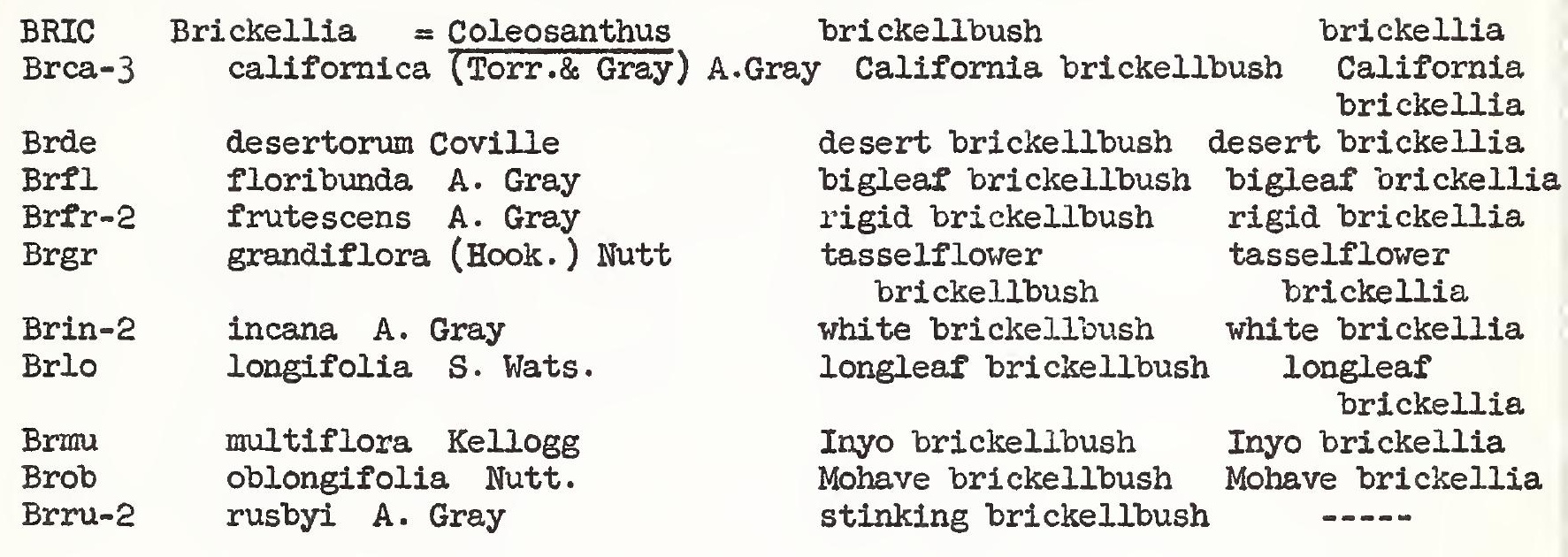

$\begin{array}{ll}\text { BUNE } & \text { Bumelia } \\ \text { Bula } & \text { lanuginosa (Michx.) Pers. }\end{array}$

Bular rigida A. Gray

gum bumelia

gum bumelia.

NOTE: Neither SPN nor the Forest Service Check List gives a common name for this variety; stifftwig gun bumelia is suggested.

BURS Bursera

Bufa fagaroides (H.B.K.) Engler

bursera.

Bumi microphylla A. Gray

Fragrant bursera

elephanttree elephant bursera

Caesalpinia gilliesii See Poinciana gilliesii

CALL

Caer

Cahu

Care

Caho

\section{Calliandra}

eriophylla Benth.

humilis Benth.

reticulata A. Gray

Canotia holacantha Torr. calliandra

false-mesquite

dwarf calliandra

netvein calliandra calliandre

false-mesquite/
CARL Carlowrightia

Caar-1 arizonica

Cali-2 linearifolia (Torr.) A. Gray canotia (crucifixion-thorn)

NOTE: The true crucifixion thorn is Zizyphus spina-christi of the Levant.

carlowrightia

Arizona carlowrightia

heath carlowrightia 


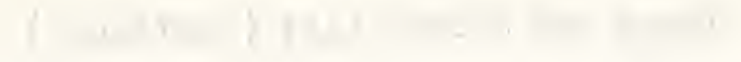

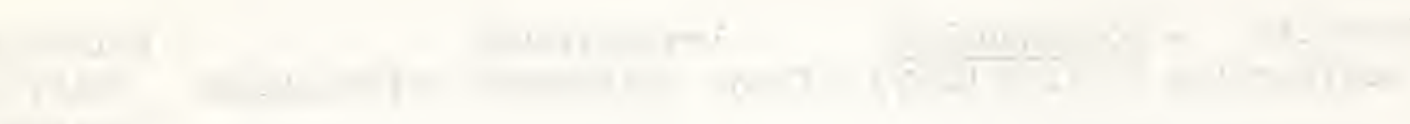
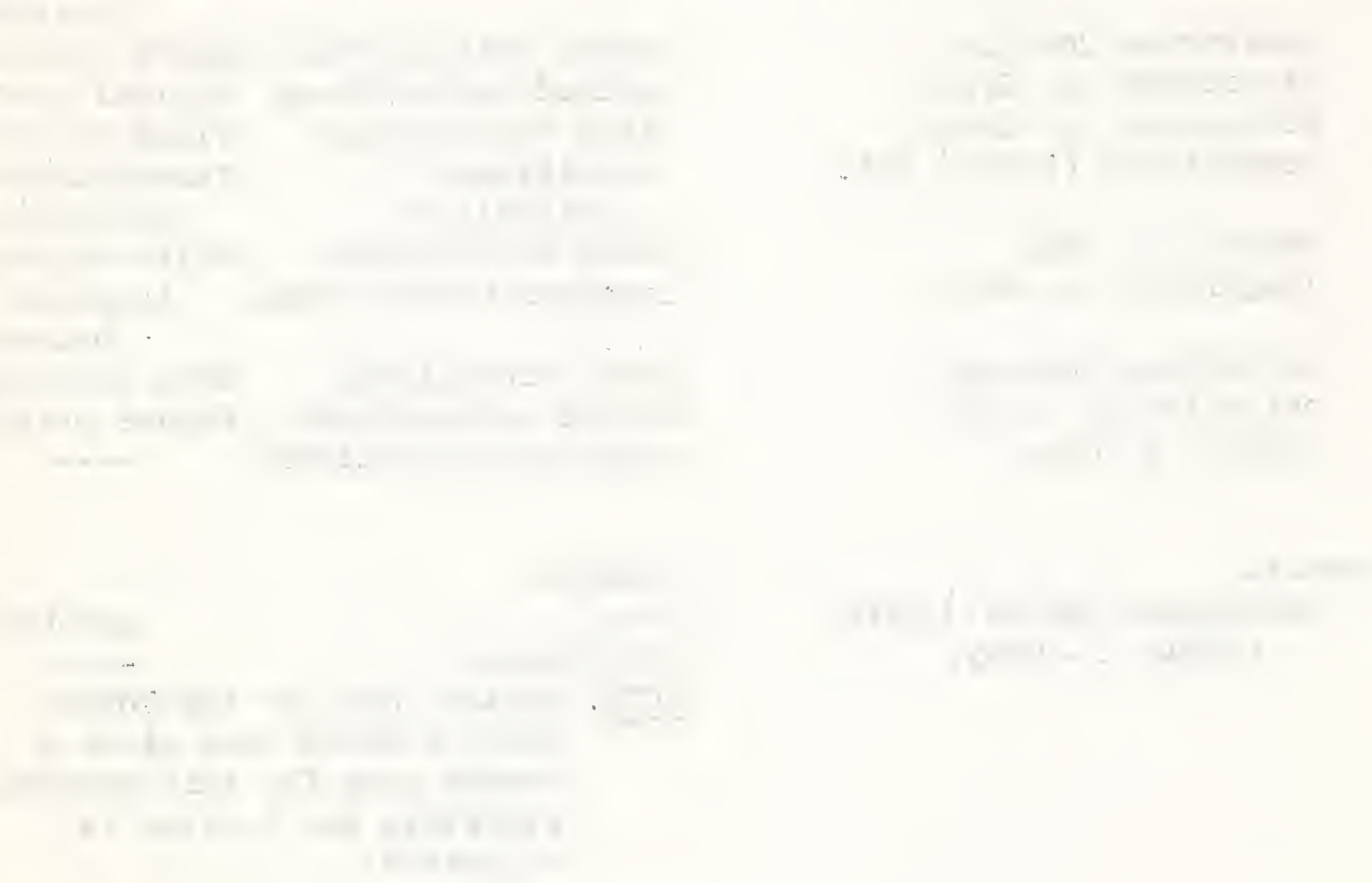

1. .

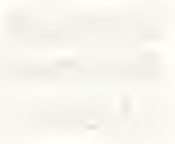

$\left(\frac{10}{2}\right.$
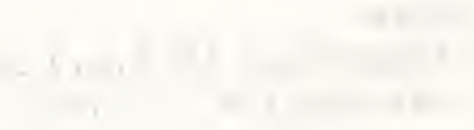

(n)

1.
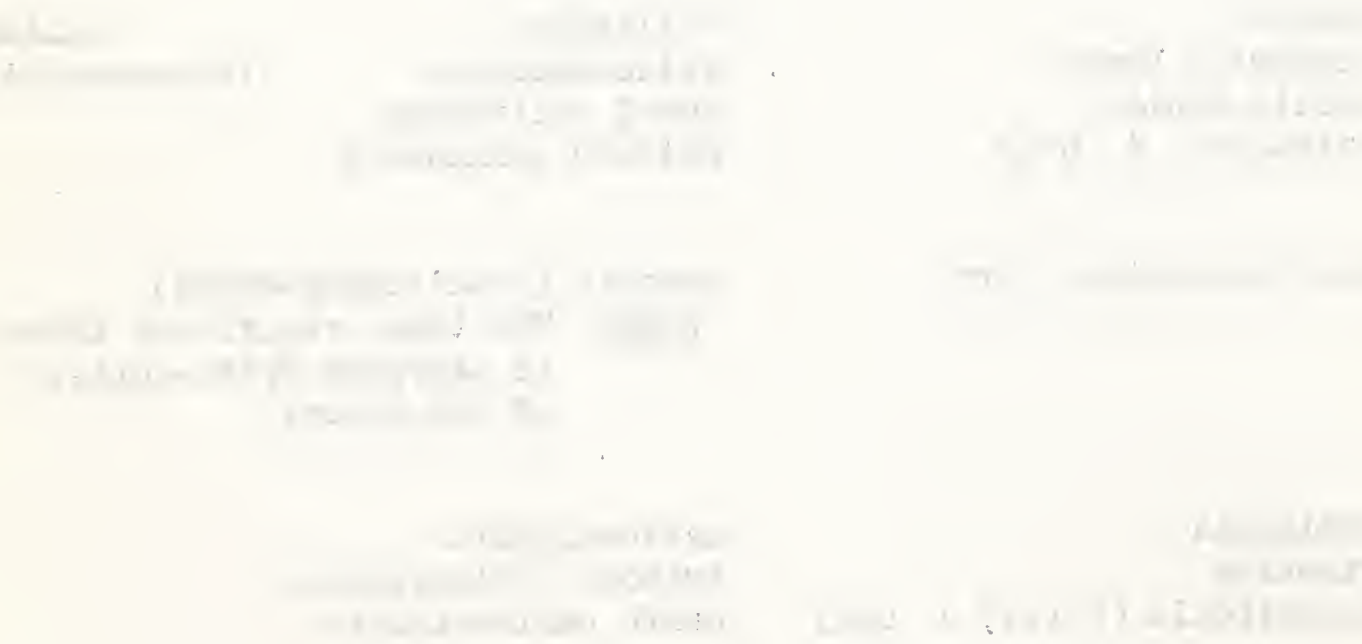
Trees and Shrubs List (continued)

$\begin{array}{lcl}\text { CAsS } & \text { Cassia See also under Forbs. } & \text { senna } \\ \text { Caar-2 } & \text { armata S. Wats. } & \text { desert senna } \\ \text { Cole-2 } & \text { leptocarpa Benth. } & \text { slimpod senna } \\ \text { Cawi } & \text { wislizenii A. Gray } & \text { Wislizenus senna }\end{array}$

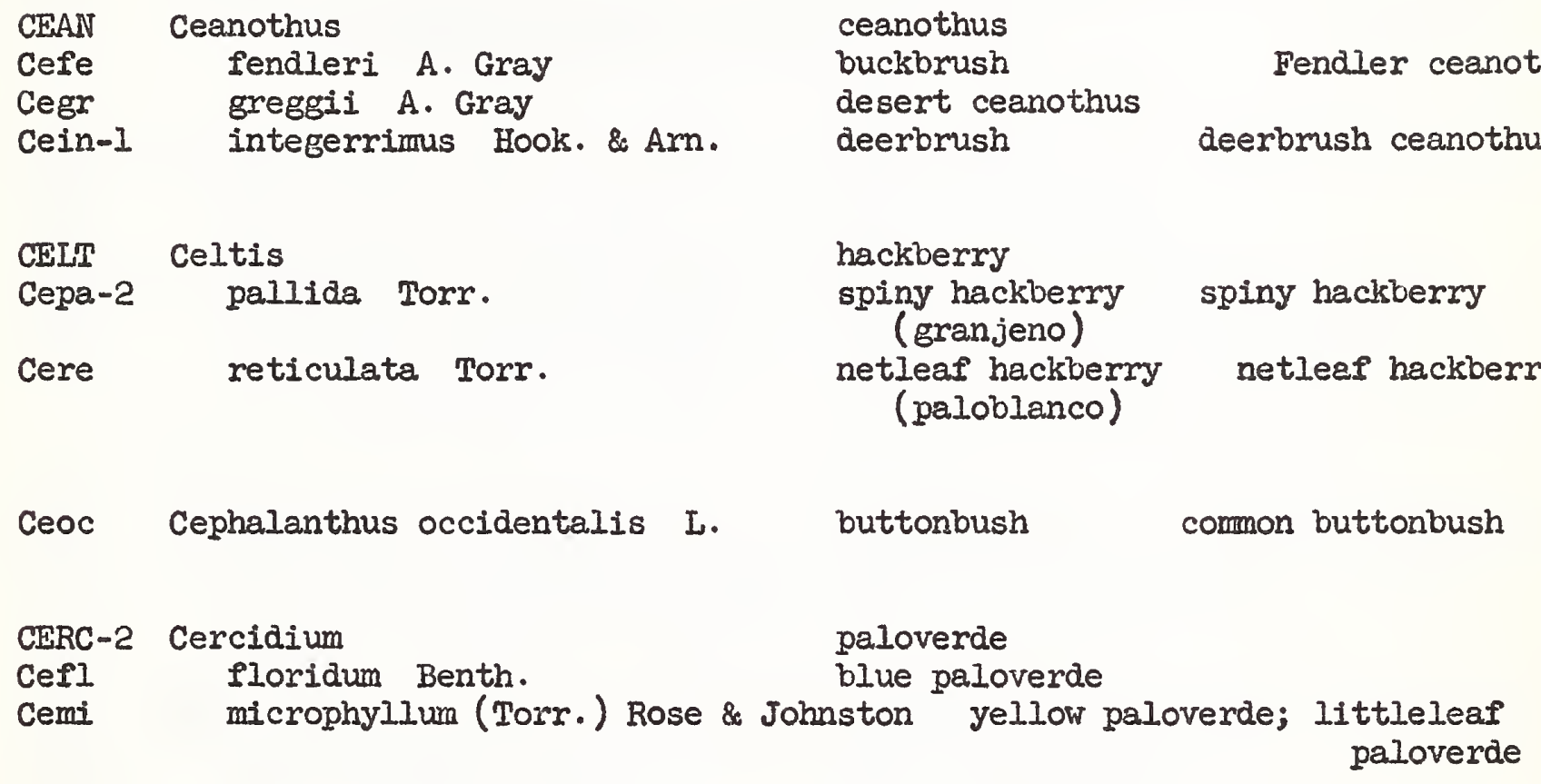

Ceoc 01 Cercis occidentalis Torr. California redbua

CERC-1 Cercocarpus mountainmahogany

NOTE: See Check List. The Forest Service has malntalned that "mahogany" should be restricted to Swletenla.

$\begin{array}{llll}\text { Cebe-1 } & \text { betuloides Nutt. } & \text { birchleaf mountainmahogany birchleaf } \\ \text { Cebr } & \text { breviflorus A. Gray } & \text { hairy mountainmahogany } & \text { hairy " } \\ \text { Cein-2 } & \text { intricatus s. Wats. } & \text { littleleaf " } & \text { littleleaf " } \\ \text { Cele } & \text { ledifolius Nutt. } & \text { curlleaf " } & \text { curlleaf " } \\ \text { Cemo } & \text { montanus Raf. } & \text { true } & \text {...... }\end{array}$

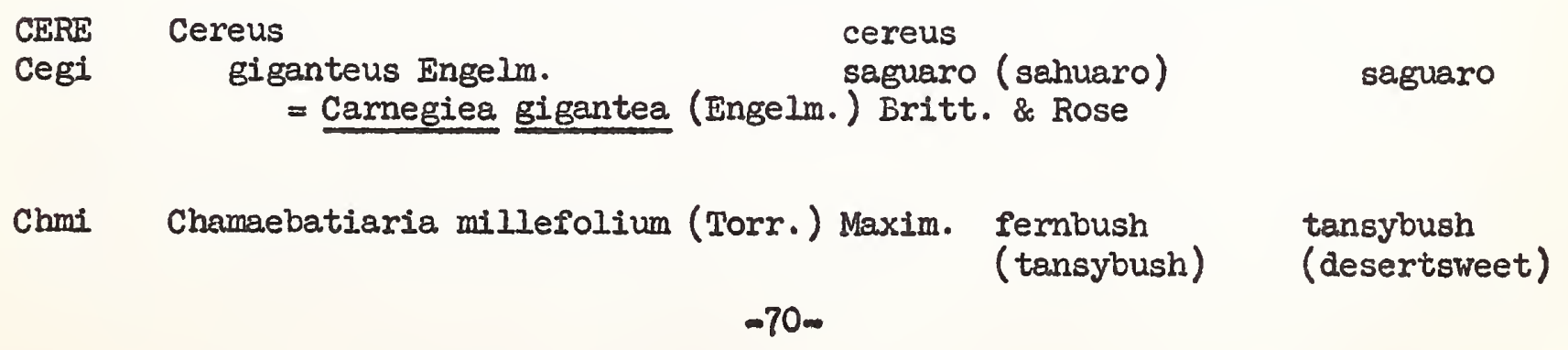


Trees and Shrubs List (continued)

Chli Chilopsis linearis (Cav.) Sweet desertwillow

\begin{tabular}{|c|c|c|c|}
\hline $\begin{array}{l}\text { CHOI } \\
\text { Char }\end{array}$ & $\begin{array}{l}\text { Choisya } \\
\quad \text { arizonica Standl. } \\
\quad=\text { C. dumosa arizonica (Stand. }\end{array}$ & $\begin{array}{l}\text { Mexican-orange } \\
\text { starleaf Mexican-orange } \\
\text { a.) I. Benson }\end{array}$ & $\begin{array}{c}\text { Arizona star- } \\
\text { lear } \%\end{array}$ \\
\hline $\begin{array}{l}\text { CHRY-I } \\
\text { Chde } \\
\text { Chna } \\
\text { Chpe-2 } \\
\text { Chpu } \\
\text { Chte } \\
\text { Chvi-3 }\end{array}$ & $\begin{array}{l}\text { Chrysothamnus } \\
\text { depressus Nutt. } \\
\text { nauseosus (Pall.) Britt. } \\
\text { paniculatus (A. Gray) H. M. Hal } \\
\text { pulchellus (A. Gray) Greene } \\
\text { teretifolius (Dur. \& Hilg.) H. } \\
\text { viscidiflorus (Hook.) Nutt. }\end{array}$ & $\begin{array}{l}\text { rabbitbrush } \\
\text { dwarf rabbitbrush } \\
\text { rubber rabbitbrush } \\
\text { II desert rabbitbrush } \\
\text { southwest rabbitbrush } \\
\text { M. Hall roundleaf rabb } \\
\text { Douglas rabbitbrush }\end{array}$ & itbrush \\
\hline CLEM & Clematis & clematis & \\
\hline C111 & Iigusticifolia IVutt. & virginsbower & $\begin{array}{c}\text { western virgins- } \\
\text { bower }\end{array}$ \\
\hline Clps & pseudoalpina (Kuntze) A. Nels. & RockyMountain clematis & \\
\hline $\begin{array}{l}\text { COID } \\
\text { Coca-2 } \\
\text { Copl }\end{array}$ & $\begin{array}{l}\text { Coldenia } \\
\text { canescens DC. } \\
\text { plicata (Torr.) Coville }\end{array}$ & $\begin{array}{l}\text { coldenia } \\
\text { spreading coldenia } \\
\text { desertmat coldenia }\end{array}$ & \\
\hline
\end{tabular}

Cora-I Coleogyne ramosissima Torr. blackbrush

Coleosanthus See Brickellia

\begin{tabular}{|c|c|c|c|}
\hline $\begin{array}{l}\operatorname{COND} \\
\operatorname{Cog} 1\end{array}$ & $\begin{array}{l}\text { Condalia } \\
\text { globosa Johnst. } \\
\text { lycioides See C. obtusifolia }\end{array}$ & $\begin{array}{l}\text { condalia } \\
\text { bitter condalia }\end{array}$ & \\
\hline $\begin{array}{l}\text { Come }-2 \\
\text { Coob }\end{array}$ & $\begin{array}{l}\text { mexicana Schlecht. } \\
\text { obtusifolia (Hook.) Weberb. } \\
\text { = } \text {. . lycioides (A.Gray) Weber }\end{array}$ & $\begin{array}{l}\text { Mexican bluewood } \\
\text { lotebush condalia } \\
\text { cb. (graythorn) }\end{array}$ & $\begin{array}{c}\text { Mexican condalia } \\
\text { lotewood condalia } \\
\text { (southwestern con- } \\
\text { dalia) }\end{array}$ \\
\hline $\operatorname{cosp}$ & spathulata & squawbush & knifeleaf condalia \\
\hline $\begin{array}{l}\text { CORN } \\
\text { Cost-1 }\end{array}$ & $\begin{array}{l}\text { Cornus } \\
\text { stolonifera Michx. }\end{array}$ & $\begin{array}{l}\text { dogwood } \\
\text { red-osjer dogwood }\end{array}$ & \\
\hline
\end{tabular}

\% Suggested common name. 

CORY-2 Coryphantha = Mammillaria, in part

Coag aggregata (Engelm.) Britt. \& Rose = Mammillaria aggregata Engelm.

Coar-2 arizonica (Engelm.) Britt. \& Rose = Mammillaria arizonica Engelm.

Coch

Coro = Mammillaria chlorantha Engelm. chlorantha (Engelm.) Britt. \& Rose foxtail cactus robustispina (Schott) Britt. \& Rose spikespine cactus = Mammillaria robustispina Schott

coryphantha golffball coryphantha Arizona coryphantha

\section{spikespine coryphantha}

\section{Covillea see Larrea}

Come-1 Cowania mexicana D. Don $=\underline{\mathrm{C}}$. stansburiana Torr.

$\begin{array}{ll}\text { CRAT } & \text { Crataegus } \\ \text { Crch } & \text { chrysocarpa Ashe } \\ \text { Crer } & \text { erythropoda Ashe } \\ \text { Crri } & \text { rivularis Nutt. }\end{array}$

CROT-2 Croton See also under FORBS Crfr fruticulosus Engelm.

CUPR Cupressus

Cuar arizonica Greene

Dasp-2 Dalea spinosa A. Gray Dasiphora See Potentilla

DASY

Date

Dawh

$$
\begin{aligned}
& \text { Dasylirion } \\
& \text { texanum Scheele } \\
& \text { wheeleri S. Wats. }
\end{aligned}
$$

Desmanthus See under FORBS

DODO

Dovi Dodonaea viscosa Jacq. cliffrose (quinine-bash, buckbrush)

hawthorn

fireberry hawthorn

Cerro hawthorn river hawthorn

croton

bush croton

cypress

Arizona cypress

smokethorn (smoketree) cliffrose 
1

1.

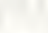

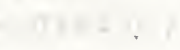

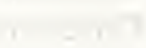

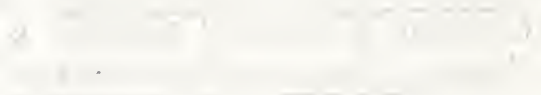

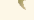

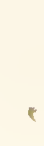

$\tau$ 
Trees and Shrubs List (continued)

\begin{tabular}{|c|c|c|}
\hline ECHI-2 & Echinocereus & echinocereus \\
\hline Eebo & boyce-thompsonii Orcutt & Boyce-Thompson echinocereus \\
\hline Ecen & engelmannii (Parry) Rümpler & Engelmann echinocereus \\
\hline Ecle & fendleri (Engelm.) Rumpler & Fendler echinocereus \\
\hline Ecpe & pectinatus (Scheidw.) Engelm. & comb echinocereus \\
\hline Ecper & $\begin{array}{l}\text { rigidissimus Engelm. } \\
=\mathrm{E} \text {. rigidissimus (Engelm }\end{array}$ & $\begin{array}{l}\text { rainbow echnicereus } \\
\text { m.) Rose }\end{array}$ \\
\hline Ecre & reichenbachi i (walp.) Haage & lace echinocereus \\
\hline Ecst & $\begin{array}{l}\text { stramineus (Engelm.) Rümpler } \\
\quad \text { = Cereus stramineus Engelm. }\end{array}$ & strawberry echinocereus \\
\hline ctr & riglochidiatus Engelm. & claretcup echinocereus \\
\hline
\end{tabular}

Ecjo Echinomastus johnsonii (Parry) Baxter; beehive cactus

= Echinocactus johnsonii Parry

= Ferocactus johnsonii (Parry) Britt. \& Rose

= Thelocactus johnsonii (Parry) W. T. Marsh

ECHI-4 Echinopsis

hedgehog-cactus

Edwinia See Jamesia

\begin{tabular}{|c|c|c|c|}
\hline $\begin{array}{l}\text { ELAE } \\
\text { ELan }\end{array}$ & $\begin{array}{l}\text { Elaeagnus } \\
\text { angustifolia I. }\end{array}$ & $\begin{array}{l}\text { Oleaster } \\
\text { Russian-olive }\end{array}$ & elaeagnus \\
\hline $\begin{array}{l}\text { ENCE } \\
\text { Enfia } \\
\text { Enfr }\end{array}$ & $\begin{array}{l}\text { Encelia } \\
\text { farinosa A. Gray } \\
\text { Prutescens A. Gray }\end{array}$ & $\begin{array}{l}\text { brittlebush } \\
\text { white brittlebush } \\
\text { green brittlebush }\end{array}$ & $\begin{array}{l}\text { encelia } \\
\text { bush encelia }\end{array}$ \\
\hline $\begin{array}{l}\text { EPHE } \\
\text { Epne } \\
\text { Eptr } \\
\text { Epvi }\end{array}$ & $\begin{array}{l}\text { Ephedra } \\
\text { nevadensis s. Wats. } \\
\text { trifurca Torr. } \\
\text { viridis Covilie }\end{array}$ & $\begin{array}{l}\text { ephedra } \\
\text { (jointfir, Morroon-tea) } \\
\text { Nevada ephedra } \\
\text { longleaf ephedra } \\
\text { green ephedra }\end{array}$ & ephedra \\
\hline $\begin{array}{l}\text { ERIO } \\
\text { Eran-2 }\end{array}$ & $\begin{array}{l}\text { Eriodictyon } \\
\text { angustifolium Nutt. }\end{array}$ & $\begin{array}{l}\text { yerba-santa } \\
\text { mountainbalm }\end{array}$ & $\begin{array}{c}\text { narrowleaf yerba- } \\
\text { santa }\end{array}$ \\
\hline
\end{tabular}

Eriogonum See under FORBS

ERYT-2 Erythrina

Erfl-3 Plabelliformis Kearney

coralbean

southwesterm coralbean western coral-

bean 


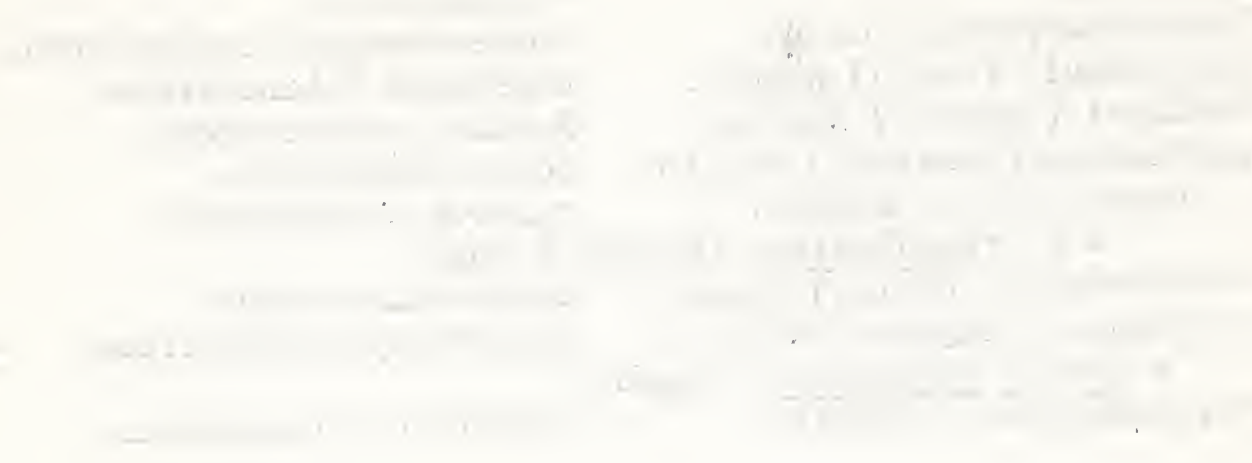

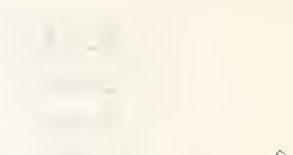


Trees and Shrubs List (continued)

$\begin{array}{ll}\text { Eura-1 Eurotia lanata (Pursh) Moq. Winterfat } & \text { (sweet-sage) winterfat }\end{array}$

Eypo Eysenhardtia polystachya (Ortega) Sarg. kidneywood

Fapa Fallugia paradoxa (D. Don) Endl. Apache-plume

FEND Fendlere.

Feru-2 rupicola A. Gray cliff Fendlerbush

FERO Ferocactus berrelcactus

Feac acanthodes (Lemaire) Britt. \& Rose Califomia barrelcactus

$\begin{array}{ll}\text { Feco } & \text { covillei Britt. \& Rose Coville barrelcactus } \\ \text { Fewi } & \text { wislizenii (Engelm.) Britt. \& Rose southwest barrelcactus }\end{array}$

(Arizona watercactus)

Flce Flourensia cernua DC. tarbush American tarbush

FORE Forestiera = Adelia Michx., NOT L. forestiera

Fone neomexicana A. Gray wild-olive forestiera New-Mexican

Foph phillyreoides (Benth.) Torr. desert-olive forestiera

forestiera

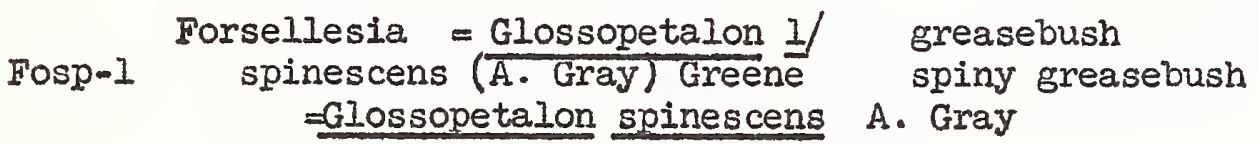

Fosp-2 Fouquieria splendens Engelm. ocotillo

FRAX Fraxinus ash

Fran anomala Torr. singleleaf ash

Franl. Lowellil (Sarg.) Little Lowell ash

Frcu cuspidata Torr.

Frgr greggii A. Gray

fragrant ash

Gregg ash

papillosa Lingelsh. Chihuahua ash

Frve velutina Torr.

velvet ash

If There is controversy among botanists as to whether or not Glossopetalon and Glossopetalum are both tenable. It seems clear, under Art. 70 , note 4 of Rules, that the names are "orthographic variants" and that Glossopetalon is untenable. 

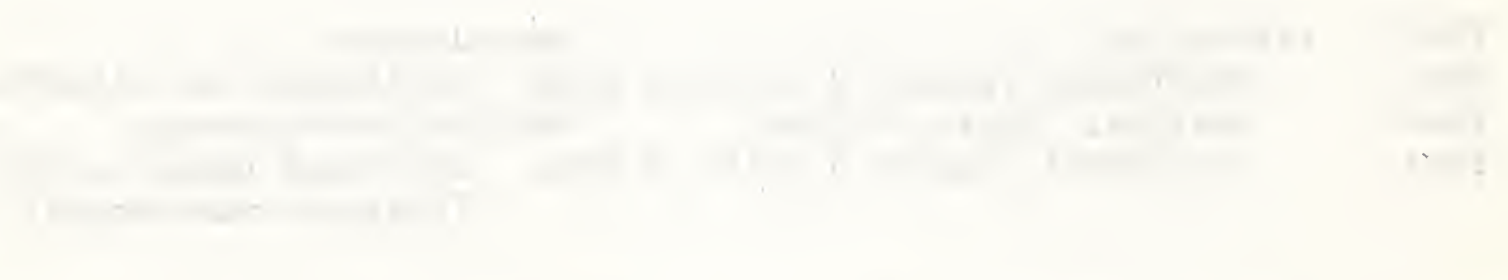

n n

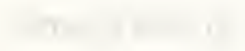
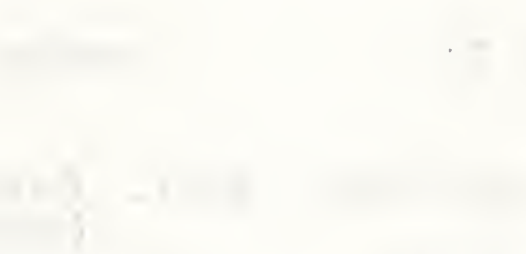

$\therefore$
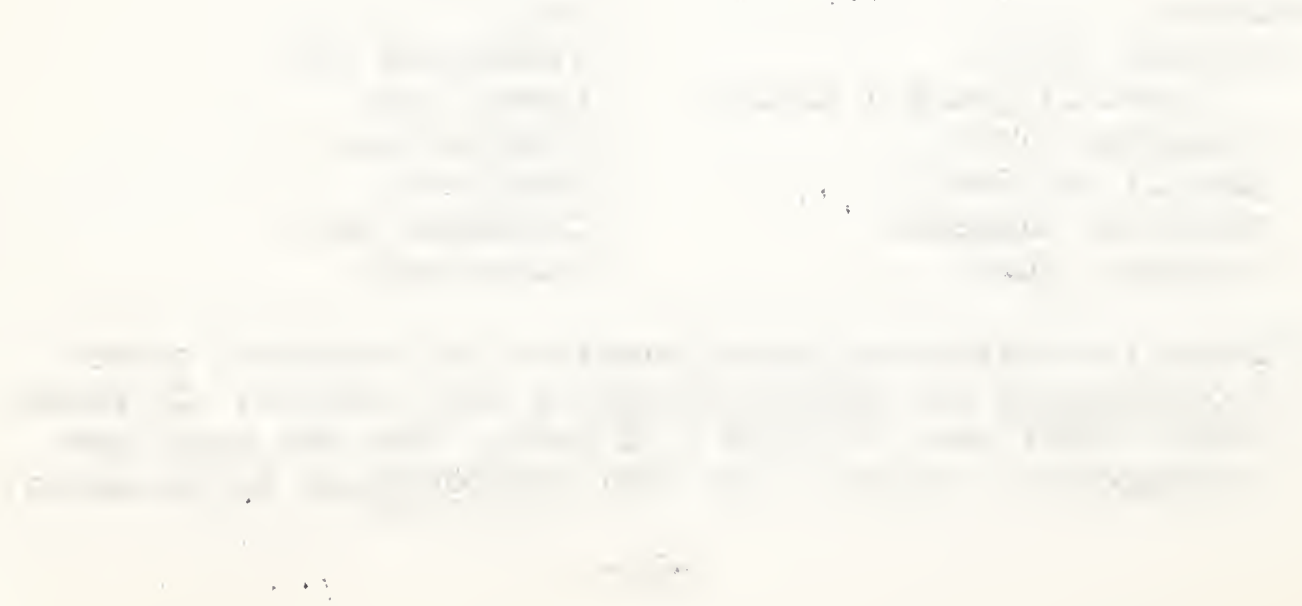
Trees and Shrubs List (Continued)

Frca Fremontia californica Torr. California fremontia

$\begin{array}{lll}\text { GARR } & \text { Garrya } & \text { silktassel } \\ \text { Gafl } & \text { flavescens s. Wats. } & \text { yellowleaf silktassel } \\ \text { Gawr-2 } & \text { Wrightil Torr. } & \text { Wright silktassel }\end{array}$

Glossopetalon See Forsellesia

\begin{tabular}{|c|c|c|c|}
\hline $\begin{array}{l}\text { Goss } \\
\text { Goth }\end{array}$ & $\begin{array}{l}\text { Gossypium } \\
\text { thurberi Todara } \\
\text { = Thurberia thespesioides }\end{array}$ & $\begin{array}{l}\text { cotton } \\
\text { desert cotton } \\
\text { (little cotton) } \\
\text { A. Gray }\end{array}$ & Thurber cotton \\
\hline $\begin{array}{l}\text { GRAY } \\
\text { Grbr } \\
\text { Grsp }\end{array}$ & $\begin{array}{l}\text { Grayia } \\
\text { brandegei A. Gray } \\
\text { spinosa (Hook.) Mog. }\end{array}$ & $\begin{array}{l}\text { hopsage } \\
\text { spineless hopsage } \\
\text { spiny hopsage }\end{array}$ & \\
\hline
\end{tabular}

Grossularia See Ribes
GUTI Gutierrezia
snakeweed
(turpentineweed, fireweed)
Guce
GuIu
Gumi
Gusa californica (DC.) Torr. \& Gray California snakeweed lucida Greene microcephala (DC.) A. Gray sticky snakeweed broom snakeweed

HIBI

\section{Hibiscus}

Hico coulteri Harv.

Hide denudatus Benth.

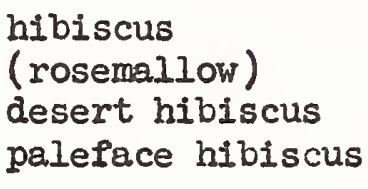

snakeweed
crucifixion-thorn
Hoem
Holacantha emoryi A. Gray

rockspiraea Hodu Holodiscus dumosus (Nutt.) Heller rockspiraea
$=$ Sericotheca dumosa (Nutt.) Rydb. (ocean-spray)

hibiscus
rosemallow
desert rosemallow
paleface rosemallow
holacantha

HMME-3 Hymenoclea

Hymo

Hysa monogyra Torr. \& Gray salsola Torr. \& Gray burrobrush singlewhorl burrobrush white burrobrush 

Trees and Shrubs List (continued)

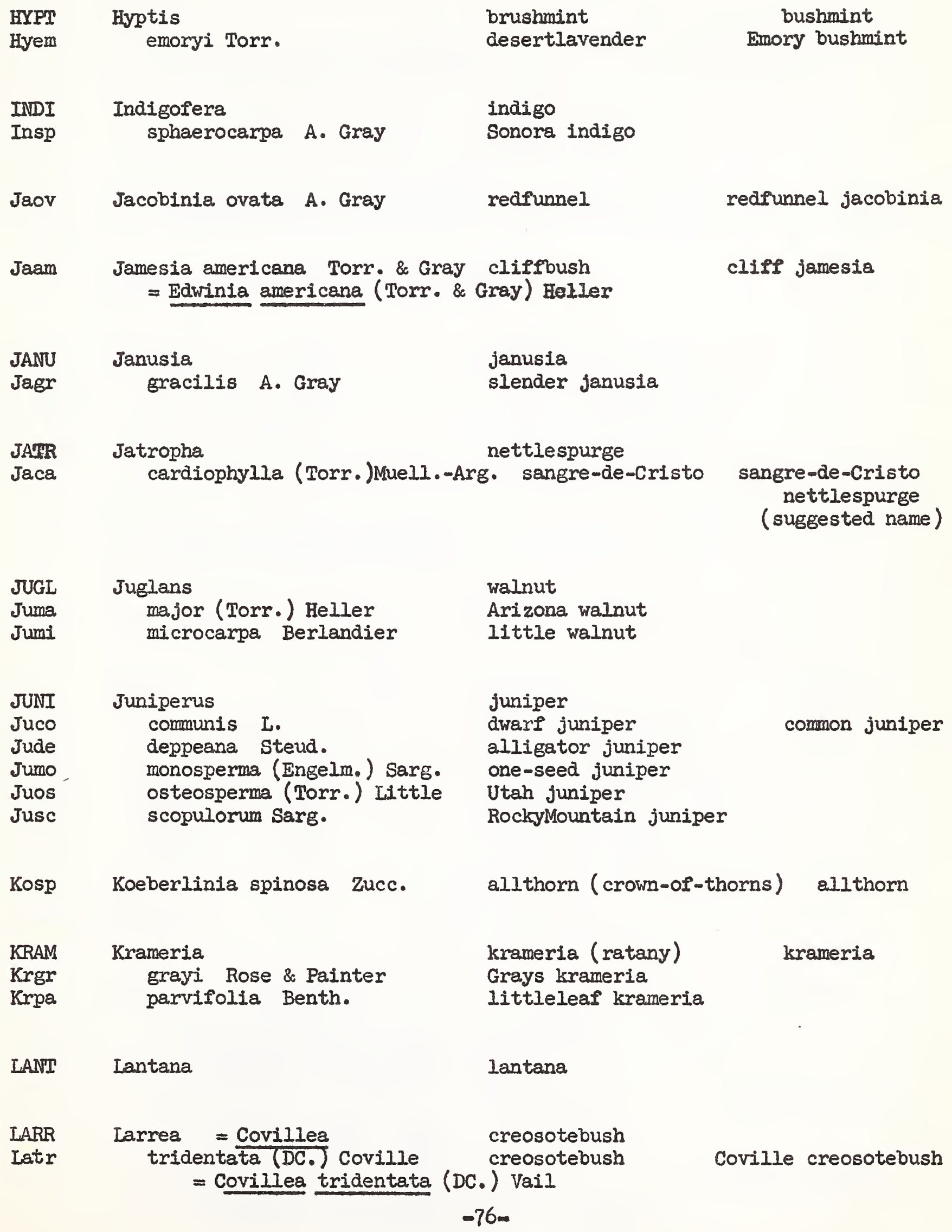




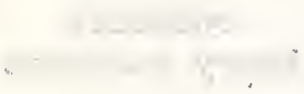
$41, y=0$
, : $: \div$

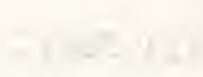

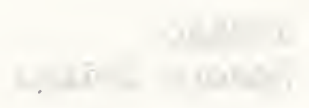
$\therefore \quad \therefore \quad=-$
$\therefore \quad+\quad$

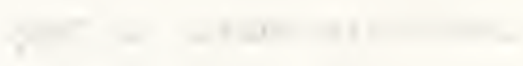

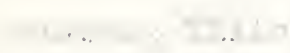

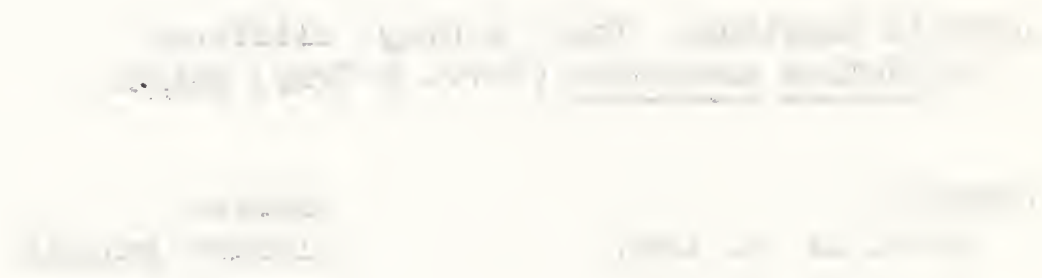
$+-$

$$
1+2+1
$$

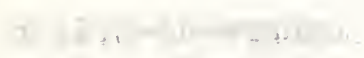

a. .
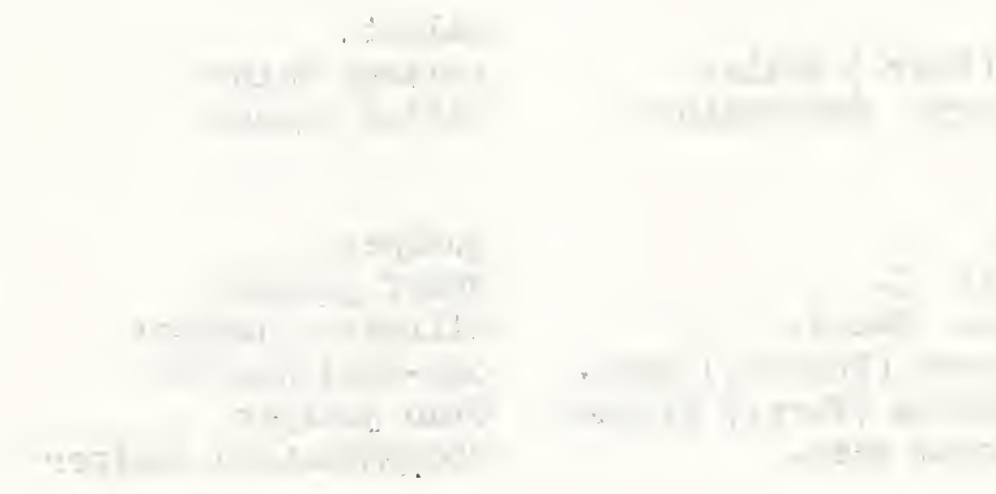

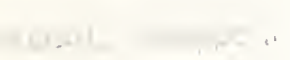

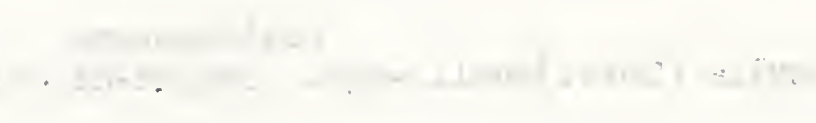

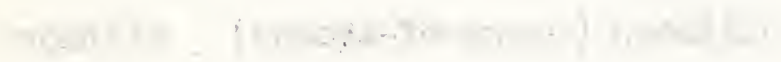

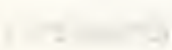
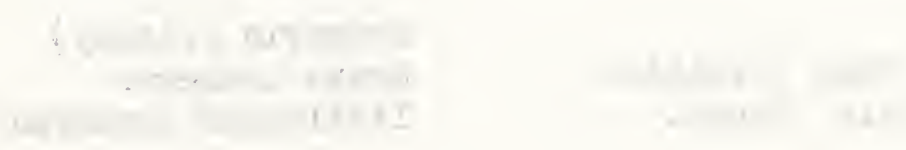

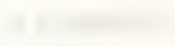

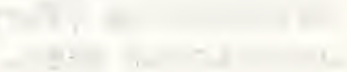

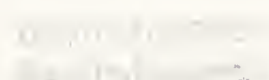

10 
Trees and Shrubs List (continued)

Lathyrus See under Forbs

Leth Iemaireocereus thurberi (Engelm.) Britt. \& Rose organpipe cactus

Lefr Lepidium fremontii s. Wats. desert pepperweed

Lepu Leptodactylon pungens (Torr.) Nutt. granite gilia = Gilia pungens (Torr.) Benth. (shrubby-phlox)

Iippia wrightii See Aloysia wrightii

$\begin{array}{lll}\text { LONI } & \text { Lonicera } & \text { honeysuckle } \\ \text { Loar } & \text { arizonica Rehder } & \text { Arizona honeysuckle } \\ \text { Loin-1 } & \text { interrupta Benth. } & \text { chaparral honeysuckle } \\ \text { Loin-2 } & \text { involucrata (Richards.) Banks bearberry honeysuckle } \\ \text { Loja } & \text { japonica Thunb. } & \text { Japanese honeysuckle } \\ \text { Lout } & \text { utahensis S. Wats. } & \text { Utah honeysuckle }\end{array}$

Lose Lophocereus schottii (Engelm.) Britt. \& Rose sinita = Cereus schottii Engelm.

LOPH Lophophora peyote

\begin{tabular}{|c|c|c|}
\hline IYCI & cium & $\begin{array}{l}\text { wolfberry } \\
\text { (desertthorn) }\end{array}$ \\
\hline Lyan & andersonii A. Gray & Anderson wolf'berry \\
\hline Iyeo & cooperi A. Gray & Cooper wolftberry \\
\hline Lyfr & eremontii A. Gray & Fremont wolfberry \\
\hline Lype-1 & pallidum Miers & pale wolfberry \\
\hline Iура,-2 & parishii A. Gray & Parish wolfoerry \\
\hline Lyto & torreyi A. Gray & Torrey wolfberry \\
\hline
\end{tabular}

IYSI-2 Iysiloma $\quad$ lysiloma
MAHO Mahonia = Berberis, in part $\ldots-\infty$ mahonia
Maha haematocarpe (Woot.) Fedde red mahonia = Berberis haematocarpa Woot.
$\begin{array}{lll}\text { Mare } \quad=\text { Odostemon haematocarpus (Woot.) Heller } & \text { (Wepens (Lindl.) G. Don } & \text { Oregongrape }\end{array}$
$=$ Berberis repens Iindl.
$=$ odostemon repens (Iindl.) Cockerell
Matr trifoliolata (Moric.) Fedde algerita
- Berberis trifoliolata Moric.
= odostemon trifoliolatus (Moric.) Heller
Iaredo mahonia




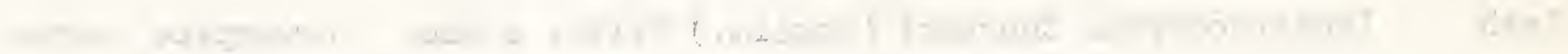

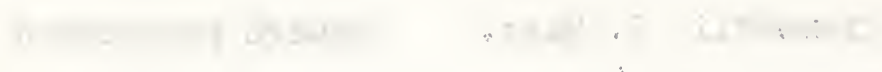

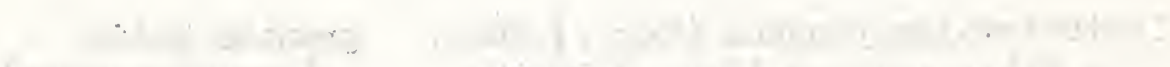

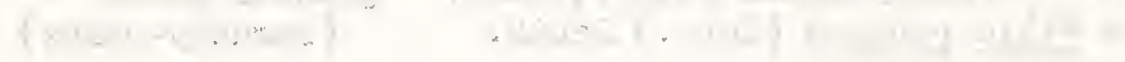

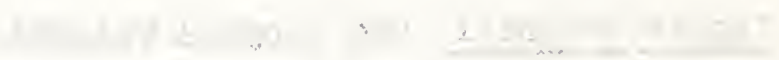

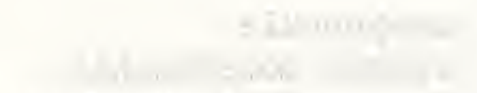

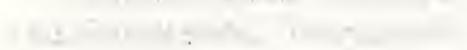

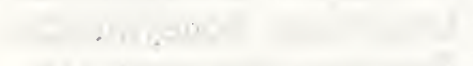

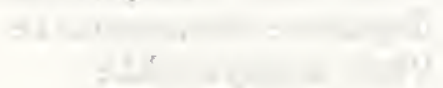
$\therefore \quad \cdots$

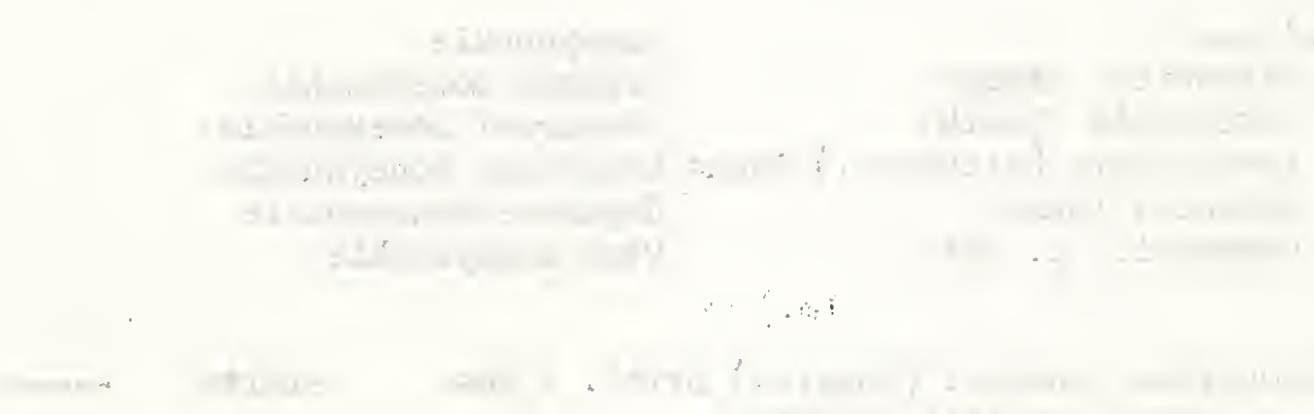

\section{$-$}
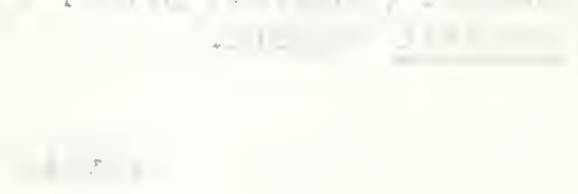

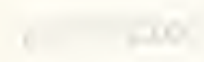
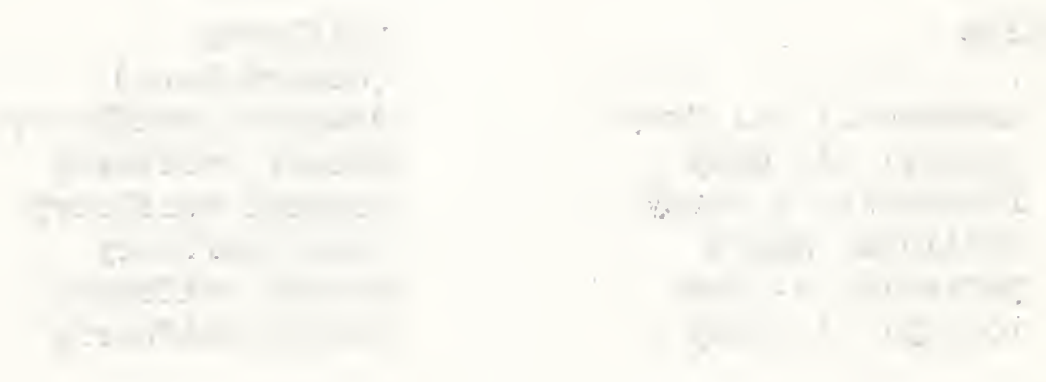
Trdes and Shrubs List (continued)

\begin{tabular}{|c|c|c|}
\hline MAMM & $\begin{array}{l}\text { Mannillaria } \\
\frac{\text { aggregata }}{\text { arizonica }} \text { See Coryphantha a } \\
\text { chlorantha See Coryphantha }\end{array}$ & $\begin{array}{l}\text { mammillaria } \\
\text { aggregata } \\
\text { arizonica } \\
\text { chlorantha }\end{array}$ \\
\hline Mafa & Pasciculata Engelm. & little fishhook mamillaria \\
\hline Mahe & heyderi Muehlenpfordt & Heyder mamillaria \\
\hline Mama-2 & macdougalii Rose & MacDougal mamillaria \\
\hline Mami & microcarpa Engelm. & fishhook mammillaria \\
\hline Maol & $\begin{array}{l}\text { Oliviae Orcutt } \\
\text { robustispina See Coryphanth }\end{array}$ & $\begin{array}{l}\text { Olivia mammillaria } \\
\text { na robustispina }\end{array}$ \\
\hline Maw1 & Wilcoxii Toumey & Wilcox mamilleria \\
\hline MENO & Menodora & menodora \\
\hline Mesc-1 & scabre A. Gray & rough menodora \\
\hline Mesc-2 & scoparia Engelm. & broom menodora \\
\hline Mesp-2 & spinescens A. Gray & spiny menodora \\
\hline MTMO & Mimosa & $\begin{array}{l}\text { mimosa } \\
\text { sensitiveplant) }\end{array}$ \\
\hline & & $\begin{array}{l}\text { NOTE: Sensitiveplant is accepted } \\
\text { common name for Mimosa pudica. }\end{array}$ \\
\hline $\begin{array}{l}\text { Mibi-3 } \\
\text { Midy }\end{array}$ & $\begin{array}{l}\text { biuncifera: Benth. } \\
\text { dysocarpa Benth. }\end{array}$ & $\begin{array}{l}\text { wait-a-bit } \\
\text { velvetpod mimosa }\end{array}$ \\
\hline Mosc & Mortonia scabrella A. Gray & scurfy mortonia \\
\hline MORU & Morus & mulberry \\
\hline Moal & alba I. & white mulberry \\
\hline Momi & microphylla Buckl. & Texas mulberry \\
\hline Nigl & Nicotiana glauca Graham & tree tobacco \\
\hline NOLI & Nolina & beargrass nolina \\
\hline Nomi & microcarpa S. Wats. & $\begin{array}{l}\text { WOTE: Beargrass is the accepted } \\
\text { common name for Xerophyllum. } \\
\text { sacahuista. }\end{array}$ \\
\hline Note & texana s. Wats. & Texas beargrass \\
\hline
\end{tabular}

arizonica See Coryphantha arizonica

Mahe lasciculata ingelm.

little fishhook mamnillaria

macdougalii Rose

microcarpa Engelm.

robustispina See Coryphanthe

robustispina

MawI

Odostemon See Mahonia

Olte Olneya tesota A. Gray

odora

scoparia Engelm.

spinescens A. Gray
imosa
(sensitiveplant)
Sensitiveplant is accepted common name for Mimosa pudica.
Mibi-3 biunciferg: Benth. dysocarpa Benth.

alba $I$.

microphylla Buckl.

texana s. Wats.

Opulaster See Physocarpus tesota

(ironwood)

NOTE: Ironwood has been applied to so many different species and genera that it is not a 


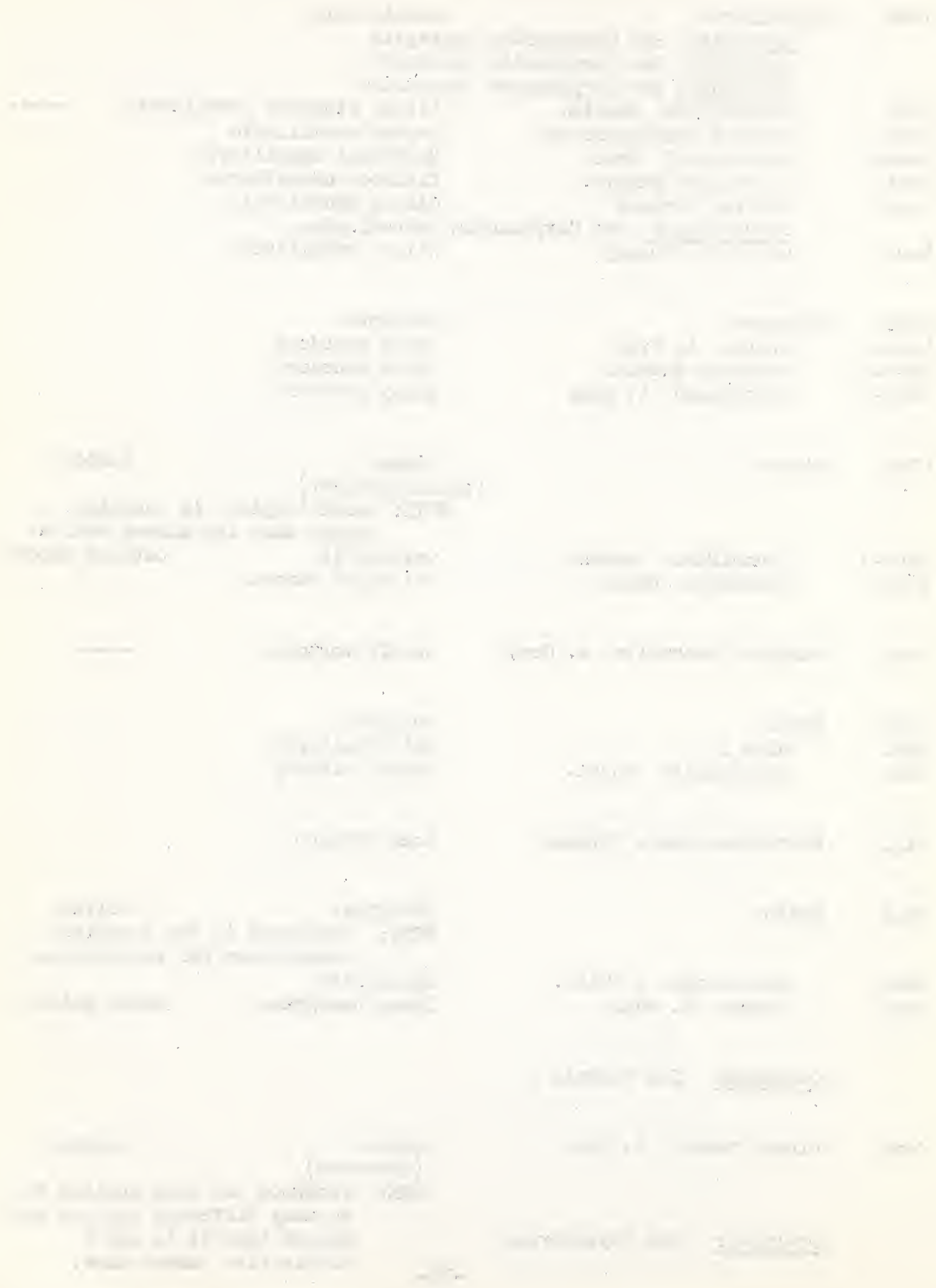


Trees and Shrubs List (continued)

\begin{tabular}{|c|c|c|c|}
\hline OPUN & Opuntia & \multicolumn{2}{|c|}{$\begin{array}{c}\text { pricklypear-- name for flat-stemed spp. } \\
\text { cholla -- name for cylindrical-stemmed } \\
\text { spp. }\end{array}$} \\
\hline Opac & acanthocarpe Engelm. \& Bigel & - buckhorn cholla & \\
\hline Oper & arbuscula Engeim. & pencil cholla & $-\infty-\infty$ \\
\hline Opau & aurea Baxter & golden pricklypear & \\
\hline Opba & basilaris Engelm. & beaverteil cactus & $\begin{array}{c}\text { beavertail prickly- } \\
\text { pear }\end{array}$ \\
\hline Opbi & bigelovil EngeIm. & jumping cholla & $\begin{array}{l}\text { Arizona jumping } \\
\text { pricklypear }\end{array}$ \\
\hline Opch & chlorotica Engelm. \& Bigel. & pancakepear cactus & $\underset{\text { pear }}{\text { dollarjoint pricikly- }}$ \\
\hline Opec & echinocarpa Engelm. \& Bigel. & strawtop & $\begin{array}{c}\text { strawtop prickly } \\
\text { pear }\end{array}$ \\
\hline Open & engelmannii Salm-Dyck & Engelmann pricklype & \\
\hline Oper & erinacea Engelm. \& Bigel. & grizzlybear prickly. & pear \\
\hline Opfr & fragilis (Nutt.) Haw. & brittle pricklypear & \\
\hline opfu & fulgida Engelm. & $\begin{array}{l}\text { cholla } \\
\text { (canecactus) }\end{array}$ & $\begin{array}{l}\text { Sonora jump- } \\
\text { lng cholla }\end{array}$ \\
\hline Opla & Iaevis Coult. & spineless cactus & smooth pricklypear \\
\hline Ople & leptocaulis DC. & Christmas cactus I & tesajo \\
\hline Oppa & parishil orcutt & Parish cholla & \\
\hline Oppo & polyacantha Haw. & plains pricklypear & \\
\hline Opra & ramosissima Engelm. & Holycross cholla & \\
\hline Opsa. & $\begin{array}{r}\text { santa-rita (Grifiths \& } \\
\text { Hare) Rose }\end{array}$ & Santa Rita cactus & $\begin{array}{l}\text { SantaRita } \\
\text { pricklypear }\end{array}$ \\
\hline Opst & stanlyi Engelm. & Stanly cholla & \\
\hline Opte & tetracantha Toumey & fourspine cholla & \\
\hline Opve & versicolor Engelm. & deerhorn cholla & $-\infty-\infty$ \\
\hline opwh & whipplei Engelm. \& Bigel. & Whipple cholle & \\
\hline
\end{tabular}

$\begin{array}{lcl}\text { OSTR } & \text { Ostrya } & \text { hophombeam } \\ \text { Oskn } & \text { knowltonil Coville } & \text { Knowlton hophornbeam }\end{array}$

PACH Pachistima (also spelled Pachystima) boxleaf

Pamy myrsinites (Pursh) Raf. myrtle boxleaf

Paac Parkinsonia aculeata I. Jerusalem-thorn (mountain-lover)

(horsebean)

pachistima myrtle pachistima Jerusalem-thorn rubberbush mariola parthenium mariola parthenium
PART-1 Parthenium

Pain-1 incanum H.B.K.

PART-2 Parthenocissus

Pain-2 inserta (Kerner) K. Fritsch creeper

thicket creeper

If This is a well-established name for Zygocactus truncatus, which blooms at Christmas, and which nearly every housewife has. 


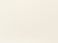



Pegr-2 Peniocereus greggii (Engelm.) Britt.\&
Rose cactus

nightblooming-cereus deerhorn

NOTE: Nightblooming-cereus is the accepted common name for the genus Hylocereus.

PENS Penstemon See also under Forbs

Pemi-2

microphyllus A. Gray

littleleaf penstemon

PHIL-2

Philadelphus

Phmi

$$
\text { microphylius A. Gray }
$$

mockorange

littleleaf mockorange

PHOR Phoradendron

Phca-4 californicum Nutt.

Phfl

Phju

Plavescens (Pursh) Nutt.

juniperinum Engelm.

mistletoe

mesquite mistletoe

Christmas mistletoe

juniper mistletoe
American-mistletoe mesquite Americanmistletoe

Christmas Americanmistletoe juniper Americanmistletoe

PHYL

Phem

Phyllodoce

mountainheath empetriformis (J.E.Smith)D.Don red mountainheath
PHYS-2 Physocarpus = Opulaster ninebark
Phmo
monogynus (Torr.) Coult.
mountain ninebark
= Opulaster monogynus (Torr.) Kuntze

\begin{tabular}{|c|c|c|}
\hline $\begin{array}{l}\text { PICE } \\
\text { Pien-1 } \\
\text { Pipu }\end{array}$ & $\begin{array}{l}\text { Picea } \\
\text { engelmannii Parry } \\
\text { pungens Engelm. }\end{array}$ & $\begin{array}{l}\text { spruce } \\
\text { Engelmann spruce } \\
\text { blue spruce }\end{array}$ \\
\hline $\begin{array}{l}\text { PINU } \\
\text { Plar } \\
\text { Pice } \\
\text { Pied } \\
\text { Pien-2 } \\
\text { Pifl }\end{array}$ & $\begin{array}{l}\text { Pinus } \\
\text { aristata Engelm. } \\
\text { cembroides Zucc. } \\
\text { edulis Engelm. } \\
\text { engelmannii Carr. } \\
\text { flexilis James }\end{array}$ & $\begin{array}{l}\text { pine } \\
\text { bristlecone pine } \\
\text { Mexican pinyon } \\
\text { pinyon } \\
\text { Apache pine } \\
\text { limber pine }\end{array}$ \\
\hline $\begin{array}{l}\text { Piflr } \\
\text { Pile: } \\
\text { Pilec } \\
\text { Pimo } \\
\text { Pipo }\end{array}$ & $\begin{array}{l}\text { reflexa Engelm. } \\
\text { leiophylla Schlecht. \& Cham. } \\
\text { chiluahuana (Engelm.) Shaw } \\
\text { monophylla Torr. \& Frem. } \\
\text { ponderosa Lawson } \\
\text { arizonica (Engelm.) Shaw }\end{array}$ & $\begin{array}{l}\text { Chihuahua pine } \\
\text { singleleaf pinyon } \\
\text { ponderosa pine } \\
\text { (western yellow pine } \\
\text { Arizona pine }\end{array}$ \\
\hline & & \\
\hline
\end{tabular}




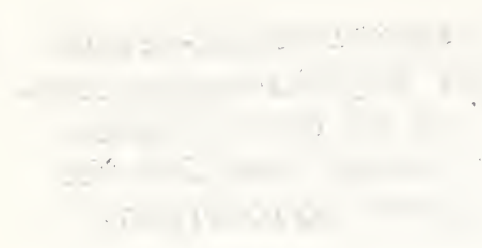

,

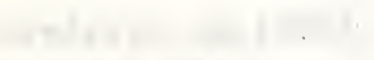

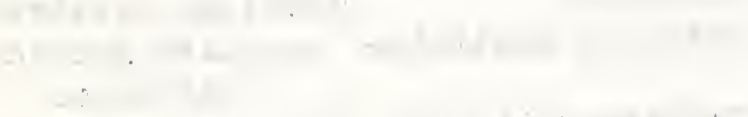

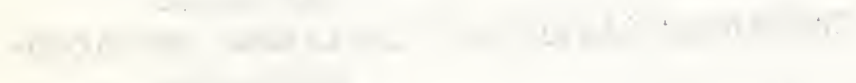

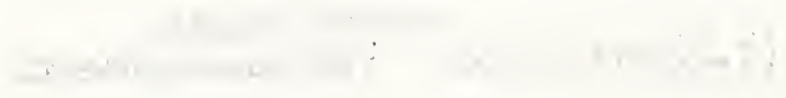

$1.1=$

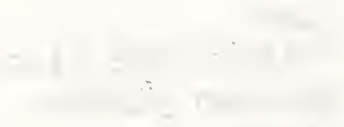

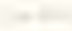

$-1$

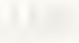

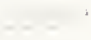

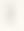

1

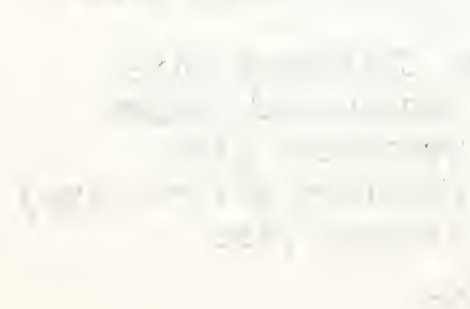




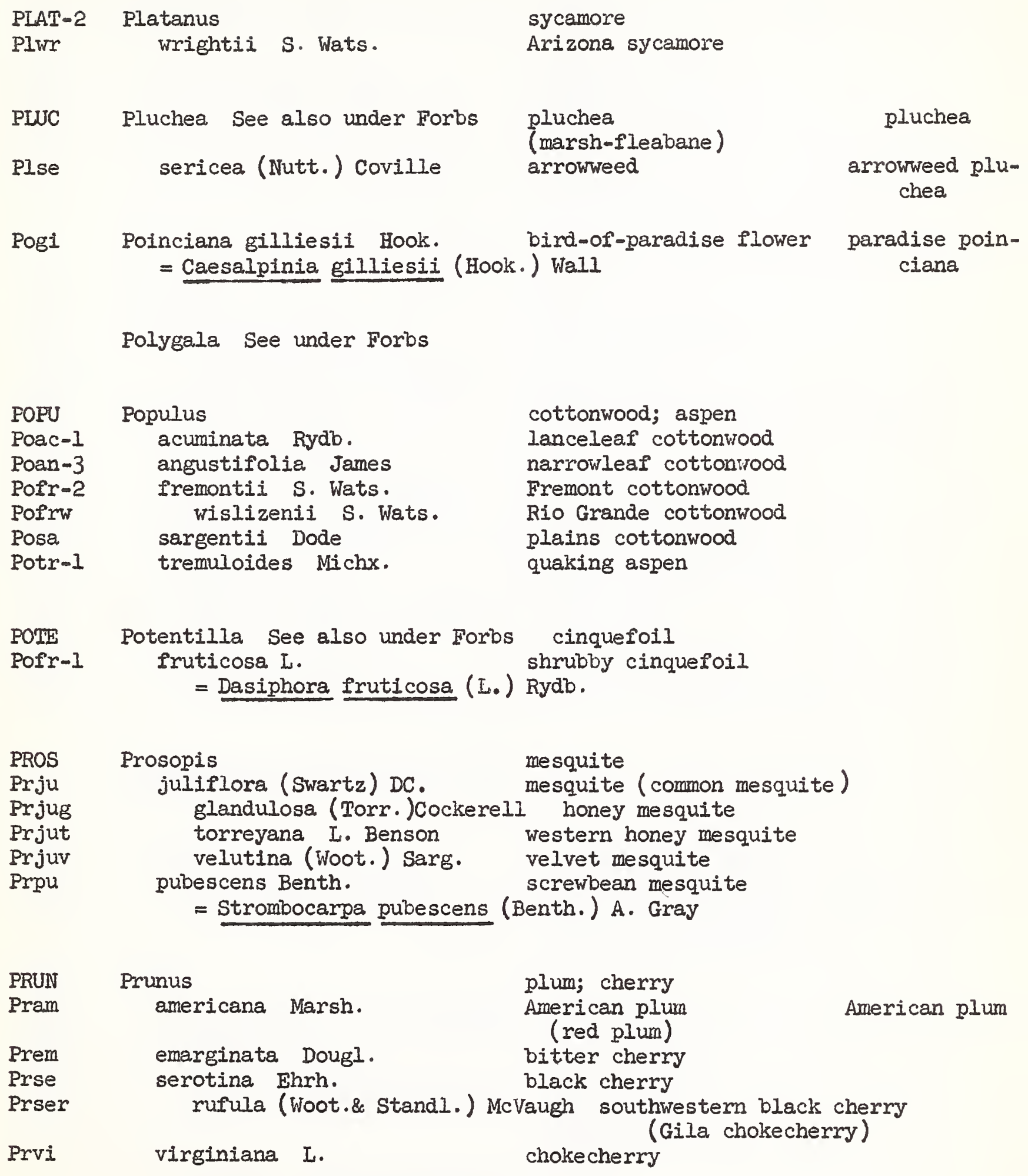

Psme Pseudotsuga menziesii (Mirb.) Franco Douglas-fir Psmeg glauca (Beissn.) Franco 


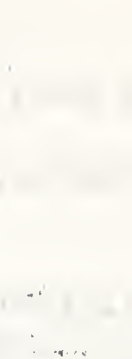

4

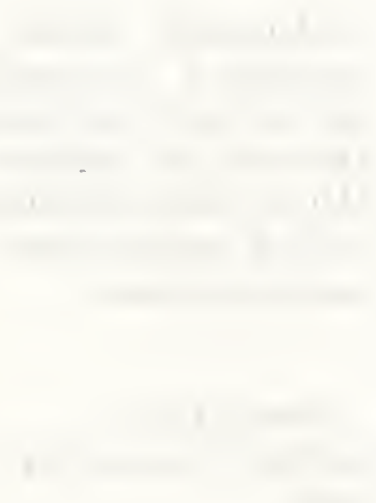


Trees and Shrubs List (continued)

Psilostrophe see under Forbs paperflower

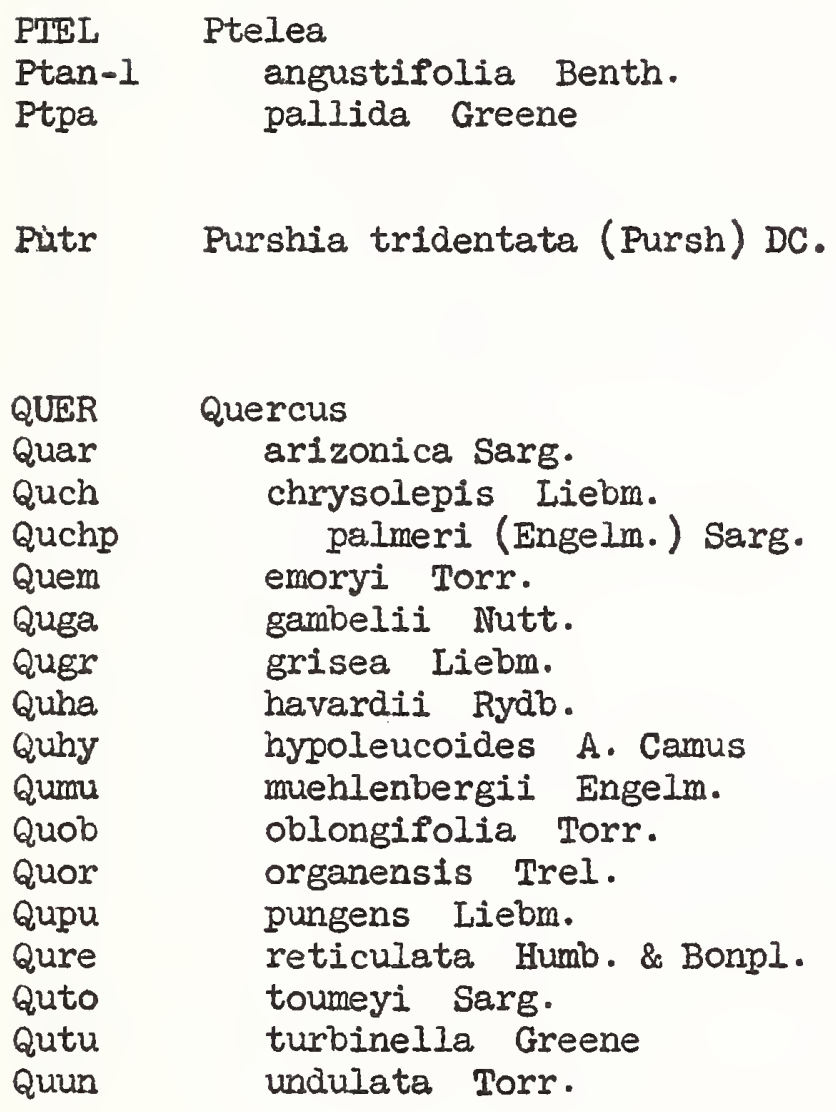

Razoumowskia See Arceuthobium hoptree narrowleaf hoptree pale hoptree

bitterbrush

(antelopebrush)

antelope bitterbrush

oak

Arizona white oak

canyon live oak

Palmer oak

Emory oak

Gambel oak (shin oak, scrub oak)

gray oak

Havard oak

silverleaf oak

chinkapin oak

Mexican blue oak

Organ Mountains oak

sandpaper oak

netleaf oak

Toumey oak

shrub live oak

wavyleaf oak (scrub oak)
RHAM

Rhbe

Rhca

Rher

RHUS

Rhch

Rhgl

Rhke

Rhla

Rhov

Rhtr
Rhamnus

betulaefolia Greene

californica Eschsch.

crocea Nutt.

Rhus

choriophylia Woot. \& Standl.

glabra $\mathrm{L}$.

kearneyi Barkley

Ianceolata (A. Gray) Britt.

ovata S. Wats. buckthorn

birchleaf buckthorn

California buckthorn (coffeeberry)

hollyleaf buckthorn

(redberry buckthorn)

sumac

Mearns sumac

smooth sumac

Kearney sumac

prairie sumac

sugar sumac

(mountain-laurel)

radicans See Toxicodendron radicans trilobata Nutt. skunkbush (squawberry) 


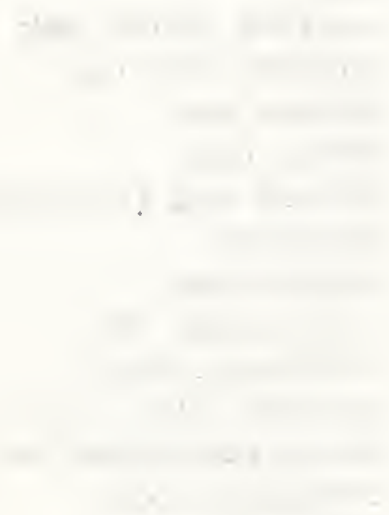

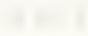

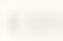


Trees and Shrubs List (Continued)

$\begin{array}{ll}\text { RIBE } & \text { Ribes }=\text { Grossularia } \\ \text { Riau } & \begin{array}{l}\text { aureum Pursh } \\ \text { cereum Dougl. } \\ \text { Rice }\end{array} \\ \text { Riin-1 } & \text { inebrians Iindl. } \\ \text { Riin-2 } & \text { inerme Rydb. } \\ \text { Rile } & \text { leptanthum A. Gray } \\ \text { Rimo } & \text { montigenum McClatchie } \\ \text { Ripi } & \text { pinetorum Greene } \\ \text { Riqu } & \text { quercetorum Greene } \\ \text { Rive } & \text { velutinum Greene } \\ \text { Rivi } & \text { viscosissimum Pursh } \\ \text { Riwo } & \text { wolfii Rothrock }\end{array}$

ROBI

Rone-I

ROSA

Roar

Rofe
Robinia

neomexicana A. Gray arizonica Rydb.

fendleri Crepin currant; gooseberry

golden currant

wax currant

squaw currant

whitestem gooseberry

trumpet gooseberry

gooseberry currant

orange gooseberry

oakwoods gooseberry

desert gooseberry

sticky currant

Rothrock currant

locust

New-Mexican locust rose

Arizona rose

Woods rose
Fendler rose

NOTE: If, as some botanists (such as Rehder) prefer,

this is a variety of Woods rose ( $R$. Woodsii)
the common name should be Fendler Woods rose.

this is a variety of Woods rose ( $R$. Woodsii)
the common name should be Fendler Woods rose. the common name

Roma

Rone-2

Rost

RUBU

Ruar

Rule

Rune

Rupa.

Rupr

Rust manca Greene neomexicana cockerell stellata Wooton
Fubus
arizonensis Focke leucodermis Dougl. neomexicanus A. Gray parviflorus Nutt. procerus P. J. Muell. strigosus Michx.

$\begin{array}{ll}\text { SAII } & \text { Salix } \\ \text { Saam } & \begin{array}{c}\text { amygdaloides Anderss. } \\ \text { bebbiana Sarg. } \\ \text { Sabe }\end{array} \\ \text { Sabo } & \text { bonplandiana H.B.K. } \\ \text { Saca-2 } & \text { caudata (Nutt.) Heller } \\ \text { Saex } & \text { exigua Nutt. } \\ \text { Sage } & \text { geyeriana Anderss. } \\ \text { Sago } & \text { gooddingil Ball } \\ \text { Sain } & \text { interior Rowlee } \\ \text { Sala-1 } & \text { laevigata Bebb } \\ \text { Sala-2 } & \text { lasiandra Benth. }\end{array}$

\begin{abstract}
Mancos rose
New-Mexican rose

desert rose
\end{abstract}

blackberry; raspberry, etc.

Arizona dewberry

whitebark raspberry

New-Mexican raspberry

western thimbleberry

Himalaya blackberry

red raspberry

American red raspberry

\author{
willow \\ peachleaf willow \\ Bebio willow \\ (beaked willow) \\ Bonpland willow \\ whiplash willow \\ coyote willow \\ Geyer willow (silver willow) \\ Goodding willow (black willow) \\ sandbar willow \\ red willow \\ Pacific willow
}


Trees and Shrubs List (Continued)

\begin{tabular}{|c|c|c|c|}
\hline & Salix (continued) & & \\
\hline Sala-3 & lasiolepis Benth. & arroyo willow (white & willow) \\
\hline Sali & ligulifolia Ball & strapleaf willow & \\
\hline Salu & lutea Nutt. & yellow willow & \\
\hline Sapa & $\begin{array}{l}\text { padophylla Rydb. } \\
\quad=\text { S. pseudomonticola Ball }\end{array}$ & serviceberry willow; & park willow \\
\hline Sasc & scoulēriana Barratt & Scouler willow & (mountain willow) \\
\hline Sata & taxifolia H.B.K. & yewleaf willow & \\
\hline SALV & Salvia see also under Forbs & sage & \\
\hline Saca-1 & carnosa Dougl. & desert sage & \\
\hline Samo & mohavensis Greene & Mohave sage & \\
\hline Sapi & pinguifolia (Fern.) Woot. \& & Standl. blue sage & $-\infty-\infty$ \\
\hline SAMB & Sambucus & elder & \\
\hline Sagl & $\begin{array}{l}\text { glauca Nutt. } \\
\quad=S \text {. cerulea Raf. }\end{array}$ & blueberry elder & \\
\hline Same-1 & melanōcarpa A. Gray & blackbead elder & \\
\hline Same-2 & mexicana Presl & Mexican elder & \\
\hline Save-1 & velutina Dur. \& Hilg. & velvet elder & \\
\hline SAPI-I & Sapindus & soapberry & \\
\hline Sadr & drumondii & western soapberry & \\
\hline SAPI-2 & Sapium & sapium & \\
\hline Sabi & biloculare (S. Wats.) Pax & jumping-bean sapium & \\
\hline SARC & Sarcobatus & greasewood & \\
\hline Save-2 & vermiculatus (Hook.) Torr. & black greasewood & \\
\hline
\end{tabular}

Scpo Sclerocactus polyancistrus (Engelm. \& Bigel.) Britt. \& Rose pineapple-cactus

Senecio See under Forbs

Sericotheca See Holodiscus

SHEP

Shar

Shca

Shro
Shepherdia argentea (Pursh) Nutt. canadensis (I.) Nutt. rotundifolia parry buffaloberry

silver buffaloberry

russet buffaloberry

roundleaf buffaloberry 


\section{$-2=0+11+\ldots$ (1)

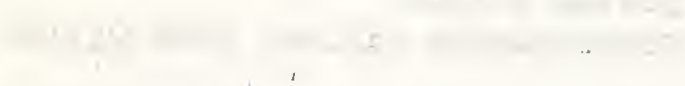

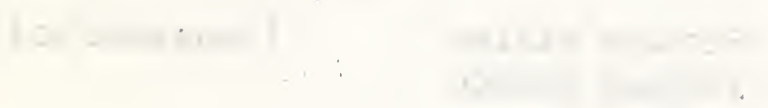
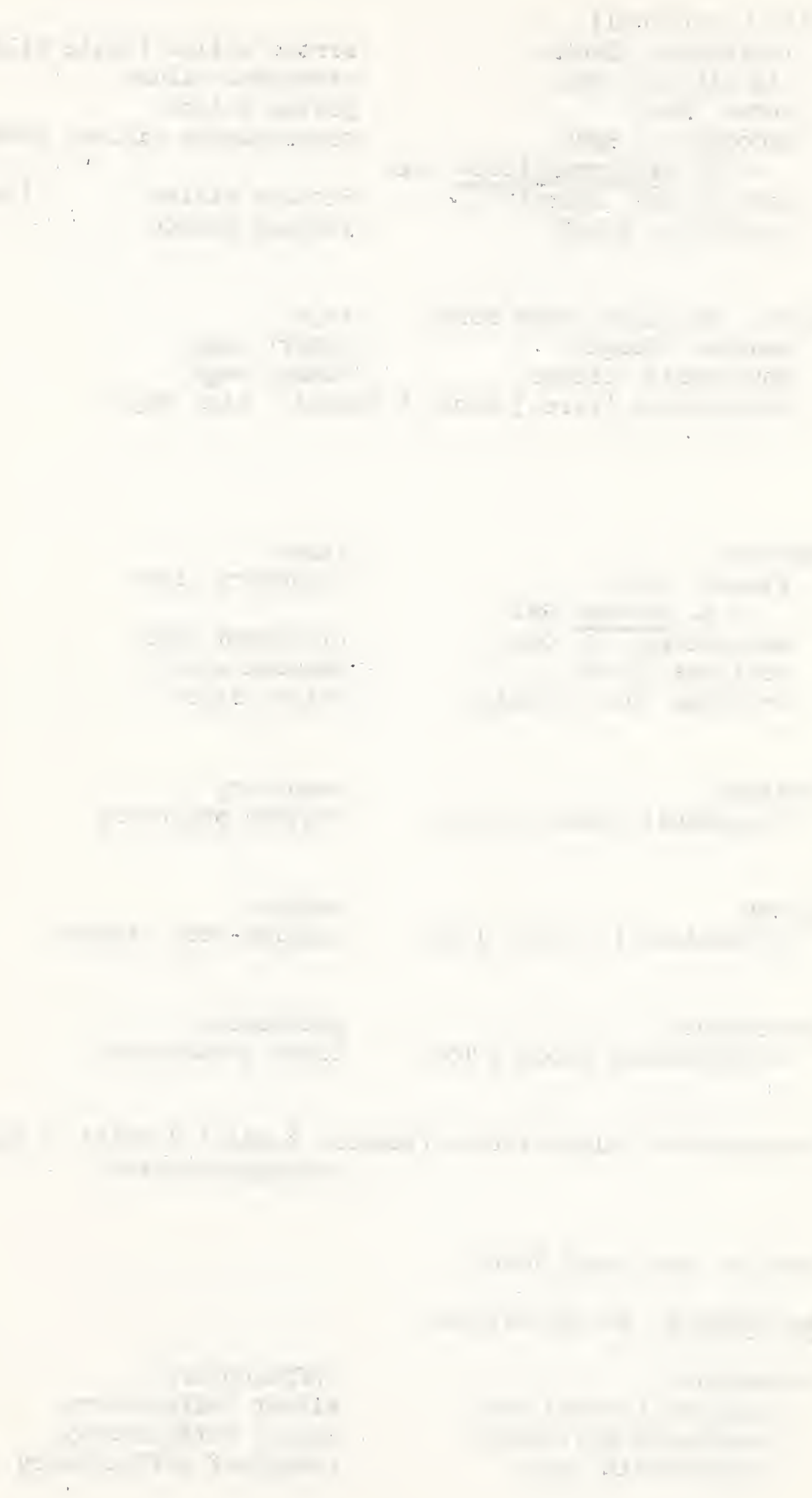$$
1
$$ 
Trees and Shrubs List (Continued)

\begin{tabular}{|c|c|c|}
\hline $\begin{array}{l}\text { SIMM } \\
\text { Sich }\end{array}$ & $\begin{array}{l}\text { Simmondsia } \\
\text { chinensis (Link) Schneid. }\end{array}$ & $\begin{array}{l}\text { jojoba } \\
\text { California jojoba }\end{array}$ \\
\hline $\begin{array}{l}\text { SOPH } \\
\text { Soar } \\
\text { Sose-1 } \\
\text { Sose-2 } \\
\text { Sost }\end{array}$ & $\begin{array}{l}\text { Sophora } \\
\text { arizonica S. Wats. } \\
\text { secundiflora (Ortega) Lag. } \\
\text { sericea Nutt. } \\
\text { stenophylla A. Gray }\end{array}$ & $\begin{array}{l}\text { sophora } \\
\text { Arizona sophora } \\
\text { mescalbean sophora } \\
\text { silky sophora } \\
\text { fringeleaf sophora }\end{array}$ \\
\hline $\begin{array}{l}\text { SORB } \\
\text { Sodu } \\
\text { Sosc } \\
\text { Stst }\end{array}$ & $\begin{array}{l}\text { Sorbus } \\
\text { dumosa Greene } \\
\text { scopulina Greene } \\
\text { Stenolobium stans (L.) Seem. } \\
=\text { S. inclsum Rose \& Standl. } \\
\quad=\text { Tecoma stans (L.) H.B.K. }\end{array}$ & $\begin{array}{l}\text { mountainash } \\
\text { Arizona mountainash } \\
\text { Greenes mountainash } \\
\text { trumpetbush }\end{array}$ \\
\hline
\end{tabular}

mescalbean

Florida yellowtrumpet

Strombocarpa pubescens See Prosopis pubescens

SYMP Symphoricarpos

Sylo

Syor

Syor-1

Syro-1

Syut

Tape Tamarix pentandra PaIl. snowberry-- white fruited species coralberry -a red fruited species longflower snowberry Indian coralberry Indiancurient coralmountain snowberry 1 , berry. roundleaf snowberry Utah snowberry

tamarisk (saltcedar) fivestamen tamarisk

Tecoma stans See Stenolcibium stans NOTE: True Tecoma species are ornamental Australian vines.

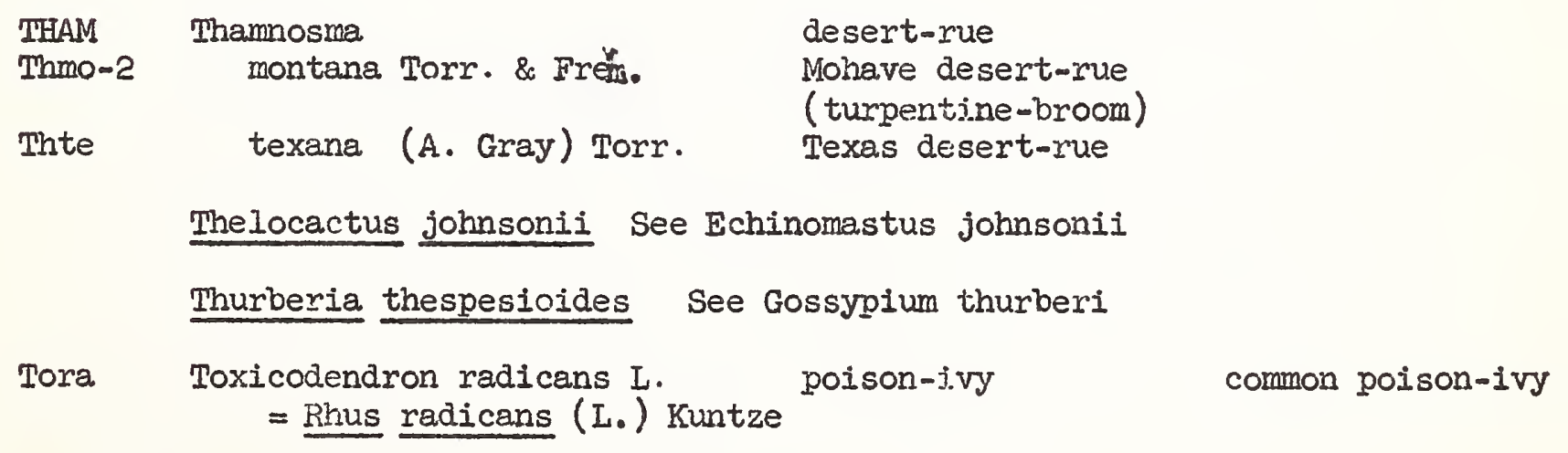

Trca-2 Trixis californica Kellogg American trixis 


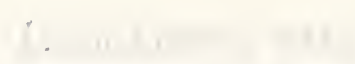

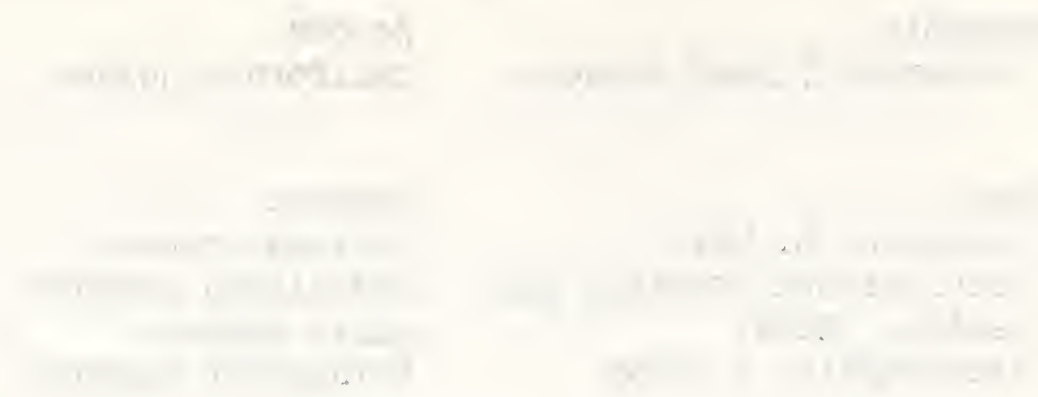

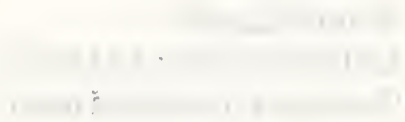

$-+\frac{1}{2}$

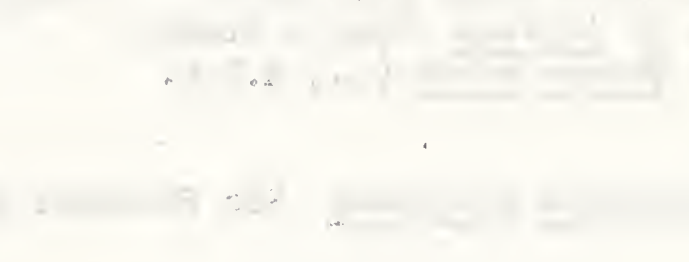

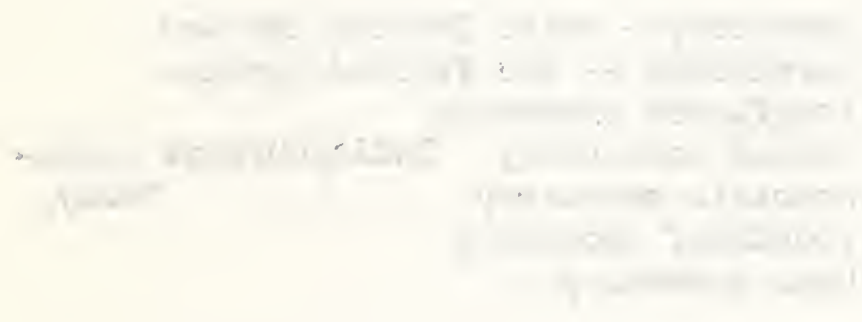

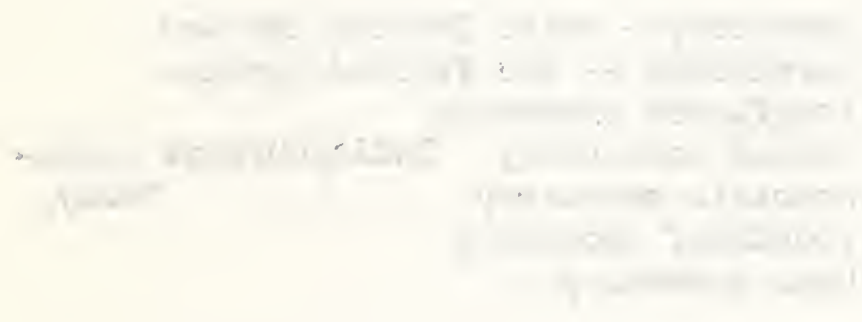

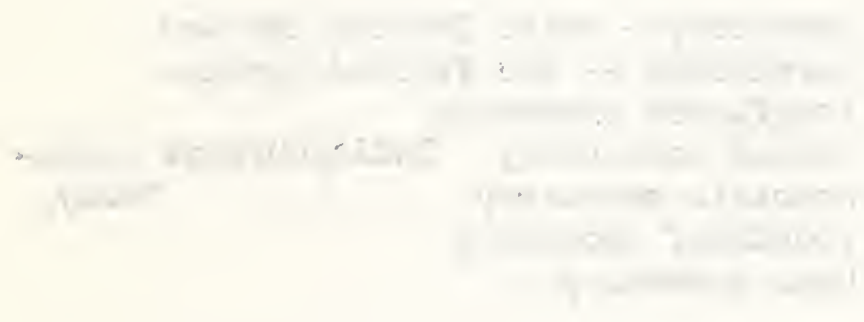

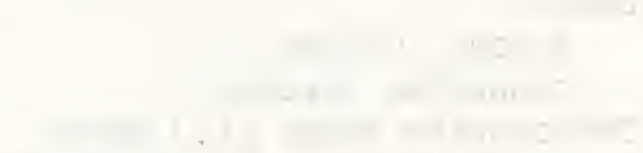

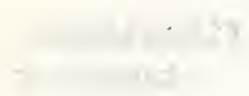

$$
+\frac{20}{2+2}+
$$

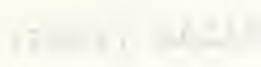

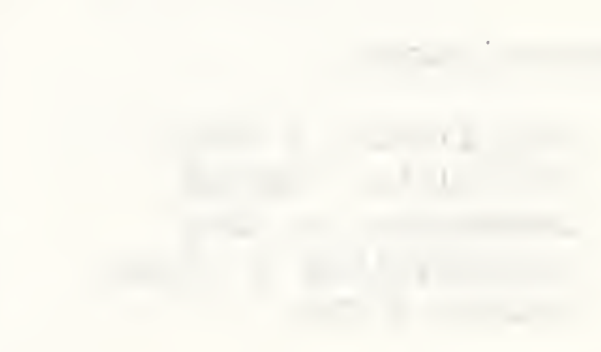


Trees and Shrubs List (Continued)

\begin{tabular}{|c|c|c|c|}
\hline Unsp & Ungnadia speciosa Endl. & Mexican-buckeye & \\
\hline VACC & Vaccinium & blueberry; whortleberry & \\
\hline Vace & cespitosum Michx. & dwarf blueberry & \\
\hline Vaor & oreophilum Rydb. & RockyMountain whortleber & \\
\hline Vasc & scoparium Leiberg & grouse whortleberry & \\
\hline \multirow[t]{2}{*}{ Vaca } & Vauquelinia californica (Torr.) & ) Sarg. Arizona-rosewood & Torrey vauque- \\
\hline & Viguiera See under Forbs & & \\
\hline VITI & Vitis & grape & \\
\hline Viar & arizonica Engelm. & canyon grape & \\
\hline Wafi & Washingtonia filifera (Iinden) & California palm & $\begin{array}{l}\text { California wash } \\
\text { ingtonia }\end{array}$ \\
\hline YUCC & Yucca & $\begin{array}{l}\text { yucca } \\
\text { (Spanish-bayonet) }\end{array}$ & yucca \\
\hline Yuan & angustissima Engelm. & fineleaf yucca & \\
\hline Yuba & baccata Torr. & blue yucca & datil yucca \\
\hline Yubr & brevifolia Engelm. & Joshua-tree & shua-tree yucca \\
\hline Yuel & elata Engelm. & soaptree yucca & \\
\hline Yugl & glauca Nutt. & smali soapweed & \\
\hline Yuha & harrimaniae Trel. & Harriman yucca & \\
\hline Yumo & $\begin{array}{l}\text { mohavensis Sarg. } \\
=Y \text {. schidigera RoezI }\end{array}$ & Mohave yucca & \\
\hline Yune & neomexicañ Woot. \& Standl. & - New-Mexican jucca & \\
\hline Yusc & schottii Engelm. & $\begin{array}{l}\text { hoary yucca } \\
\text { (Spanish-bayonet) }\end{array}$ & Schotts yucca \\
\hline Yuto & torreyi Shafer & $\begin{array}{l}\text { Torrey yucca } \\
\text { (Spanish-dagger) }\end{array}$ & \\
\hline ZIZI & Ziziphus & jujube & \\
\hline ue & Zuckia arizonica Standl. & Arizona zuckia & \\
\hline
\end{tabular}




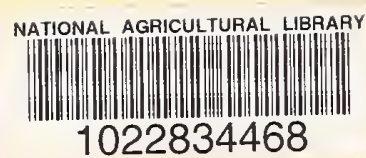

